\title{
- Separation of cohorts on the basis of bacterial type IV conjugation systems identified from metagenomic assemblies
}

3 Benjamin R. Joris, Tyler S. Browne, Thomas A. Hamilton, David R. Edgell and Gregory B. Gloor

5 Author Affiliations: Department of Biochemistry, Schulich School of Medicine, The University of Western

6 Ontario, London, Ontario, N6A 3K7, Canada

7 Corresponding author: Gregory B. Gloor, ggloor@uwo.ca 


\section{\& Abstract}

Conjugation enables the exchange of genetic elements throughout environments, including the human gut microbiome. Conjugative elements can carry and transfer clinically relevant metabolic pathways which makes precise identification of these systems in metagenomic samples clinically important. Here, we outline two distinct methods to identify conjugative systems in the human gut microbiome. We first show that conjugative systems exhibit strong population and age-level stratification. Additionally, we find that the total relative abundance of all conjugative systems present in a sample is not an informative metric to use, regardless of the method of identifying the systems. Finally, we demonstrate that the majority of assembled conjugative systems are not included within metagenomic bins, and that only a small proportion of the binned conjugative systems are included in "high-quality" metagenomic bins. Our findings highlight that conjugative systems differ between general North Americans and a cohort of North American pre-term infants, revealing a potential use as an age-related biomarker. Furthermore, conjugative systems can distinguish between other geographical-based cohorts. Our findings emphasize the need to identify and analyze conjugative systems outside of standard metagenomic binning pipelines.

\section{Importance}

The human gut microbiome is increasingly being associated with human health outcomes through shotgun metagenomic sequencing. The usual approach of metagenomic-level analyses is to bin assembled sequences into approximations of bacterial genomes and perform further investigations on the resultant bins. Here, we show that type IV conjugative systems differ between age and geographically-based cohorts and that these systems are systematically excluded by binning algorithms. We suggest that analysis of type IV conjugative systems should be added to the current metagenomic analysis approaches as they contain much information that could explain differences between cohorts beyond those we investigated.

\section{Background}

B of bact

\section{$\mathrm{p}$} kno

thar is present on the same genetic element [1]. ICEs encode their own T4SS, and can mobilize other elements 
[2]. Conjugative elements often contain antibiotic resistance genes, but also can harbour useful biosynthetic and biodegradation genes [3]. Furthermore, conjugative systems can serve as vectors to introduce CRISPR systems, metabolic pathways or novel functions into the gut microbiota [4-9]. Therefore, characterizing the full complement of conjugative systems in the human gut could expand the number of useable vectors for these applications. Precise identification of conjugative systems from metagenomic samples could also provide insights to their distribution in populations and their correlation with antibiotic exposure, age, and health status.

For a DNA sequence to be considered mobilizable, it must encode an origin of transfer (oriT) sequence that is recognized and nicked by a relaxase protein $[1,10]$. Relaxase proteins contain a conserved histidine triad that coordinates a divalent metal ion, as well as tyrosine residues that bind the oriT DNA sequence and catalyze the nicking reaction $[11,12]$. In addition to a relaxase gene and an oriT sequence, a full complement of type IV secretion system and coupling proteins are required for a sequence to be mobilizable. In the wellstudied Agrobacterium tumefaciens conjugative system, there are 12 proteins involved in the transfer of the DNA-relaxase complex from one bacterial cell to another $[13,14]$. Homologs of the VirB4 ATPase that are essential for assembly of the conjugative system and DNA transfer are generally similar to the phylogeny of the bacteria harbouring them [15] and thus are useful for classifying conjugative systems [16]. The synteny of conjugative transfer genes is also highly conserved among conjugative systems [14]. Both the synteny and presence of highly-conserved genes involved in conjugation facilitates the classification of genetic elements as potentially conjugative if the sequences are annotated as belonging to the components of the T4SS [17] (Figure 1).

Previous work has identified novel conjugative systems in the human and animal gut microbiomes, but the focus was mainly on ICEs and not on conjugative plasmids [3, 18, 19]. Identifying conjugative plasmids from a short-read metagenomic assembly is difficult for several reasons. The initial barrier is the difficulty in assembling circularized plasmids from short-read sequencing data [20]. A second barrier is that the contiguous DNA sequences (contigs) that compose metagenome-assembled genomes (MAGs) are binned together based on sequence composition and coverage. Binning of a plasmid with its cognate genome will not happen unless the contigs that compose the plasmid are maintained in the same copy-number and have the same sequence composition as the chromosome. These criteria are generally not met because conjugative systems are usually more AT rich than the cognate chromosome [1] and often do not have a unit copy number. Since nearly $80 \%$ of the non-redundant set of genomes from the human-gut microbiome are from difficult-toculture species that are known only from MAGs [21], alternate methods must be employed to assemble and identify conjugative plasmids from the metagenomic sequencing data. Computational tools have recently been developed to identify plasmids from metagenomic assemblies [22], but would be rendered pointless if applied to already binned data that systematically excludes plasmids [23]. Methods that identify conjugative systems prior to binning should be able to capture the full spectrum of ICEs and conjugative plasmids. 
Here, we show that T4SS conjugative systems can be identified using two distinct methods (Figure 2). First, we used profile HMMs (pHMMs) to identify conjugative systems directly from metagenomic assemblies of general North American and North American pre-term infant samples [17]. Second, we searched predicted protein sequences vs. UniRef90 [24] for proteins involved in conjugation to identify conjugative systems from a human gut microbiome genome set. With this approach differences between additional cohorts in the relative abundances of extracted systems could be recognized. While the differences between cohorts found using the two methods were not identical, both methods did illustrate that different age and population cohorts were distinct. Non-equivalent data between the methods suggests some level of incompleteness or bias in the conjugative systems found by one or both methods. Finally, we demonstrate that the majority of conjugative systems produced by a metagenomic assembly are not included in high-quality bins that were used to compose human gut microbiome genome sets. Our findings provide a roadmap to integrate the analysis of conjugative systems alongside the genomic content of bacteria.

\section{Methods}

\section{Assembly and identification of conjugative systems in North American short- read data}

Samples belonging to a general North American $(\mathrm{n}=50)$ and a North American pre-term infant cohort $(\mathrm{n}=51)$ were assembled de novo (Supplemental Table 1$)$. Reads from these samples were downloaded from the Sequence Read Archive using the SRA toolkit version 2.9.2, deduplicated with dedupe.sh [25], and trimmed with Trimmomatic version 0.36 [26] with options LEADING:10 TRAILING:10. Processed reads were assembled sample-by-sample using SPAdes version 3.14.0, option --meta [27]. The resultant assemblies were imported into Anvi'o version 6.0 [28] where the presence of T4SS, T4CP, and relaxase proteins were predicted using the anvi-run-hmms module, which integrates HMMER3 functionality [29]. Contigs that contained pHMM matches for all three classes of conjugative proteins were extracted and annotated by aligning open reading frames (ORFs) predicted with Prodigal version 2.6.3 [30] to the UniRef90 database [24]. Subregions of the contigs where annotations for conjugative proteins were present, with no more than 20 ORFs between successive UniRef90 annotations for conjugative proteins, were extracted. The processed read data were mapped to the extracted conjugative systems using Bowtie2 version 2.3.5 [31] with the settings --no-unal --no-mixed --no-discordant. Extraction of the subregions was to avoid an artificially high proportion of reads mapping in samples where the bacterium is present, but the ICE has not integrated in its chromosome (Supplemental Figure 1). Taxonomic prediction of the contigs was conducted with Kaiju version 1.7.2 utilizing the RefSeq non-redundant protein database [32]. MOB-suite verion 1.4.9.1 was utilized to characterize the incompatibility grouping of the conjugative system, if possible [33]. PlasFlow version 1.1.0 was used to classify whether the system was chromosomally integrated or a plasmid [22]. The proportion of 
reads mapping to the conjugative systems was extracted from the Bowtie 2 output, and the mapping data was visualized using Anvi'o [28]. Raw counts of reads mapping to the extracted conjugative systems were transformed using a centered log-ratio. The principal component coordinates of the first 2 components were used for clustering by hdbscan [34].

\section{Reference human gut metagenome set}

A near-complete and non-redundant set of human gut microbiome genomes were downloaded from the European Bioinformatics Institute FTP site (ftp://ftp.ebi.ac.uk/pub/databases/metagenomics/umgs_analyses/) [21]. These genomes were assembled from 13,133 metagenomic samples using SPAdes [27] and binned using MetaBAT2 [35]. The quality of binned genomes were assessed using CheckM [36]. High-quality genomes were defined as $>90 \%$ completeness and $<5 \%$ contamination and medium-quality genomes were defined as $>50 \%$ completeness and $<10 \%$ contamination, and these genomes were used to create the non-redundant set of genomes. The program dRep was used to cluster the genomes at $99 \%$ sequence identity [37] thereby dereplicating the genome bins, creating a set of 2505 genomes.

\section{Identifying and quantifying conjugative systems in reference human gut metagenome set}

ORFs were predicted in the genome by Prodigal version 2.6.3 [30]. The predicted protein sequences were then aligned to the UniRef90 database [24] using the Diamond protein aligner version 0.9.14 [38]. Contigs were extracted from the genomes if they contained annotations for a relaxase/mobilization protein and a type IV secretion/type IV coupling protein using a word-search strategy. MOB-suite verion 1.4.9.1 was utilized to characterize the incompatibility grouping of the conjugative system, if possible [33]. PlasFlow version 1.1.0 was used to classify whether the system was predicted to be chromosomally integrated or located on a plasmid [22]. Short-read data from 785 samples (Supplemental Table 2) were downloaded from the Sequence Read Archive using the SRA toolkit version 2.9.2. The reads were processed and aligned to the regions of the contigs where type IV conjugative systems were located using the previously described methods. The principal component coordinates of the first 3 components were used for clustering by hdbscan [34].

\section{Binning of Assemblies}

For each assembly, all 101 samples were mapped to the contigs using Bowtie2 [31]. The mapping files were sorted and indexed with SAMtools [39] and then the assemblies were binned using MetaBAT2 version 2.12.1 [35]. CheckM version 1.1.2 was used to assess the quality of the resultant bins [36]. High-quality bins were defined using the same cutoffs ( $>90 \%$ completion and $<5 \%$ redundancy) as Almeida et al (2019) 
defined. Bins not passing that threshold were classified as "low-quality". The previously identified contigs with conjugative systems were classified based on their presence in bins, and the types of bins they were present in. Results of this classification were visualized using SankeyMATIC (http://sankeymatic.com/).

\section{Results}

\section{Conjugative systems identified from assembly of short-read data distinguish North American cohorts}

51 samples from a pre-term infant cohort and 50 from a general North American cohort were assembled sample-by-sample using metaSPAdes [27] to identify T4SS conjugative systems from a full pool of assembled contigs (i.e. not binned) and to compare the relative abundances of these systems between cohorts. For these analyses, contigs with conjugative systems were defined by pHMM matches for a relaxase, a type IV coupling protein, and a type IV secretion system, which offers a fast and precise method to annotate a limited number of protein families. From the assembly of the pre-term infant cohort 96 of 470500 contigs met the criteria, whereas 268 of 15100646 contigs from the general cohort did (Supplemental Table 3). Predicted ORFs from contigs with conjugative systems were aligned to the UniRef90 database and the subregions with conjugative systems were extracted. The short-read data from all 101 samples were mapped to all 391 subregions of conjugative proteins.

The patterns of conjugative system occurrence and relative abundance in the two cohorts are distinct (Figure 3). Conjugative systems belonging to the Proteobacteria phylum were only assembled from pre-term infant samples and did not have any apparent occurrence in the general population. Furthermore, most identified conjugative contigs were private to the cohort they were assembled from. When the log-transformed principal component analysis data were clustered with hdbscan [34], the two cohorts formed separate clusters (Figure 4, Supplemental Figure 2). These findings show that North American pre-term infants have a strikingly different array of conjugative systems than the general North American cohort.

\section{Mapping human gut microbiome data from cohorts to conjugative systems re- veals distinct patterns}

Next we explored relative abundances of conjugative systems in a greater number of cohorts, without having to conduct computationally-expensive metagenomic assemblies. For this analysis, conjugative systems were identified from a set of 2505 bacterial genomes, which represent a non-redundant and near-complete picture of the human gut microbiome [21]. A total of 1598 contigs from 787 genomes that contain UniRef90 annotations for relaxase/mobilization and T4SS/T4CP proteins were identified (Supplemental Table 3). From 
these contigs, 3216 subregions where conjugative protein annotations were concentrated on the contig were extracted (Supplemental Figure 1), with 2413 being $>1 \mathrm{~kb}$ in size and used for visualization. Short-read human gut microbiome sequencing data from 785 samples, spread across 8 cohorts were aligned to the extracted subregions (Supplemental Table 1). With the conjugative systems identified from the human gut metagenome set, the two North American cohorts that were previously analyzed are still distinct, albeit in a different way (Figure 5). Only a very small number of the reads from the North American and European Infant cohorts mapped to conjugative systems. The only notable signal is in the Proteobacteria phylum for the North American pre-term infants, a finding consistent with what was found by de novo assembly. The West African and South American cohorts also share similar characteristics as both have an overall lower apparent relative abundance of conjugative systems compared to the other non-infant cohorts, particularly in the Bacteroidetes phylum. The other four cohorts appear similar with regards to the presence and absence of the conjugative systems. The cohorts separated into three distinct clusters (Figure 6, Supplemental Figure 3 ), when the principal components of the centered log-ratio transformed data were clustered using hdbscan [34]. In this analysis infant cohorts were excluded because of their extreme sparsity. The majority of the West African and South American samples clustered together consistent with Figure 5. Not readily apparent from the cladogram was the East Asian cohort that clustered primarily on its own. The North American Indigenous, North American general, and Western European general samples largely clustered together. Like the conjugative systems identified from the de novo assemblies of short-read data, the relative abundances of conjugative systems identified from a human gut metagenome set separated cohorts into distinct groups.

\section{Percentage of reads mapping to conjugative systems is inconsistent between methods}

For the cohorts that were examined using both methods of conjugative system identification, the percentages of total reads mapping to the identified conjugative systems were not equal between methods (Figure 7). From the conjugative systems identified from the general North American cohort assembly of the short reads the mean percentage of reads mapping was $0.62 \%$ (95\% CI $[0.22 \%, 1.1 \%])$, whereas the mean percentage of reads mapping to the conjugative systems identified from the genome set was $(2.69 \% 95 \%$ CI $[1.25 \%, 4.64 \%])$. A lower average percentage in the assembled dataset than the genome set conjugative systems suggests that the assemblies of the short read sequences were not able to successfully capture the full diversity of conjugative systems found in an average North American individual. Conversely, for the pre-term North American infants, the mean percentage of total reads mapping to conjugative systems identified from the assemblies was $3.33 \%(95 \%$ CI $[0.00182 \%, 2.39 \%])$, whereas from the reference genome set the mean percentage was $(0.271 \%$ $95 \%$ CI $[0.025 \%, 1.21 \%])$. In terms of the composition of the reference gut metagenome set, it is probable that the bulk of the bacterial genomes are sourced from deeply sequenced cohorts, like a general North 
American population, rather than a niche cohort like pre-term infants. This results in a more precise relative abundance estimate of conjugative systems in the general North American cohort than in the pre-term infant cohort. These findings together suggest that the total percentage of reads mapping to conjugative systems is not particularly informative and these data should be treated as compositions with relative abundances of features being compared between groups.

\section{The majority of conjugative systems identified by assembly are omitted from metagenomic bins}

The assemblies were binned using MetaBAT2 [35], which was also used to bin the MAGs in the human gut genome set used in the prior analyses[21] to further explore how conjugative systems are distributed within common metagenomic analyses. Of the 364 assembled contigs containing pHMM matches to all three protein categories, 270 were not included in any metagenomic bins (Figure 8). For the 94 contigs included in metagenomic bins, 65 of those were found in high-quality bins ( $>90 \%$ completion and $<5 \%$ redundancy). Among the 29 contigs included in bins that do not meet the aforementioned threshold, 8 are within bins that are greater than or equal to 1 megabase in size, potentially suggesting that fragments of a conjugative plasmid may have binned together.

\section{Discussion}

The relative abundances of conjugative systems identified from MAGs and isolate genomes from the human gut differ between cohorts similarly to how relative abundances of human gut MAGs of different species are differential between cohorts [40]. The infant cohorts stood out the most from the other cohorts; the infant gut microbiome is composed largely of members of the genus Bifidobacteria and is recognized as being distinct to the microbiomes of adults over the first few years of life [41]. Furthermore, we observed that the gut microbiome of pre-term infants were distinct from other infants and adults. This could be because of exposure to antibiotics from birth and colonization by opportunistic Proteobacteria pathogens such as members of the genera Escherichia, Klebsiella, and Enterobacter [42]. As shown in Figure 2a, the only conjugative systems that showed a signal for the samples belonging to the North American pre-term infants were those belonging to the Proteobacteria phylum. The degree of difference in relative abundances of conjugative systems between infants and adults suggests that conjugative systems could be a potential biomarker for age, or for the relative maturity of the infant microbiome.

The relative abundances and distributions of conjugative systems in the West African and South American cohorts were distinct from the other non-infant cohorts, which is similar to the findings in the relative abundances of bacterial species in these cohorts [40]. As well, the East Asian cohort clustered separately from 
the other non-infant cohorts. These findings suggest that conjugative systems might be useful biomarkers for other factors beyond just age and further focus on geographical or health-related differences may yet reveal additional separation between cohorts based on the relative abundances of conjugative systems.

Comparing the findings between methods in Figure 7, it is clear that the total percentage of reads mapping to conjugative systems is not an effective metric; the differences between the relative abundances in conjugative systems that were present from the genome set between the general North American and North American preterm infants did not persist when examining the relative abundances of the assembled systems. Comparing Figure 3 and Figure 5, the conjugative systems assembled in pre-term infants are almost entirely missing in the genome set. It is clear that there is a degree of incompleteness in terms of infant conjugative systems in a database consisting of primarily MAGs assembled from non-infant datasets. However, for the non-infant data, the genome set appeared to capture a larger percentage of the conjugative systems. Neither method of identifying and quantifying conjugative systems is perfect; the reference bacterial genome set may be incomplete for less commonly studied cohorts, but the sequencing depth of a single sample may not be sufficient to assemble the less abundant conjugative systems in an environment.

We found that a reference bacterial genome set can be useful for identifying coarse differences in the conjugative systems between populations; however, this method may not capture the true diversity of conjugative between populations, because many conjugative systems may be omitted. To produce MAGs, contigs generated by metagenomic assembly are typically binned using a program such as MetaBAT2 [35]. Conjugative systems are often more AT rich than the parent genomes [1], which would result in the conjugative system and cognate genome not occurring in the same metagenomic bin. Additionally, plasmids are not necessarily maintained in a unit copy number within the cell, causing differential sequence coverage in comparison to the parent genome, which results in plasmids being excluded from MAGs. Therefore to capture a more complete image of the conjugative systems present in an environment, identification of the systems must take place before binning.

The assembled contigs were binned with MetaBAT2 [35] as a way of quantifying the effect of binning, which revealed that the vast majority of the assembled conjugative systems were not included in metagenomic bins and therefore would not be included in a MAG database, which confirms recent findings [23]. Many of the binned conjugative systems were not within a bin that would pass the quality cutoff to be included in the genome set as well [21]. Interestingly, eight of the conjugative systems were binned into low-quality bins that were smaller than $<1 \mathrm{MB}$ in size, which may suggest that the fragments of a conjugative plasmid could be binned together, which would increase the completeness of the conjugative system. 


\section{Conclusions}

Conjugative systems differ between cohorts and require special consideration to ensure that they are included in analyses. ICEs and plasmids can carry harmful systems, such as antimicrobial resistance, but also can act as vectors for bile salt metabolism and for detoxification modules [3]. These cargo genes are relevant for research relating to the gut microbiome's role in pathogenicity as well as metabolism and digestion. Comprehensive identification and quantification of conjugative systems could allow for association of conjugative systems with different health outcomes. Because assembled plasmid-based conjugative systems are rarely included in metagenomic bins [23], they need to be identified and analyzed outside of standard binning pipelines. At present, it is not possible to assemble complete plasmids from short-read metagenomic data [20], so it may helpful to identify bins containing conjugative systems in an attempt to cluster the fragments of plasmids present in an assembly together. Identifying type IV conjugative systems using pHMMs or annotations and using tools such as PlasFlow [22] to identify plasmids out of a full assembly in parallel with standard binning analyses will enhance research of the associations between the human gut microbiome and human health.

In the future, improvements in assembly and binning algorithms will continue to improve the recovery of low relative abundance conjugative elements and improve the completeness and accuracy of the assembled fragments. Additionally, long-read assembly permits the circularization of genomes and plasmids [43, 44] and the binning of plasmids to their cognate genomes using methylation data [45], which will reduce the ambiguity of the origins of conjugative systems (i.e. whether they are an ICE or independently circularized plasmid) and provide a more complete picture of the cargo they carry and the differences between cohorts.

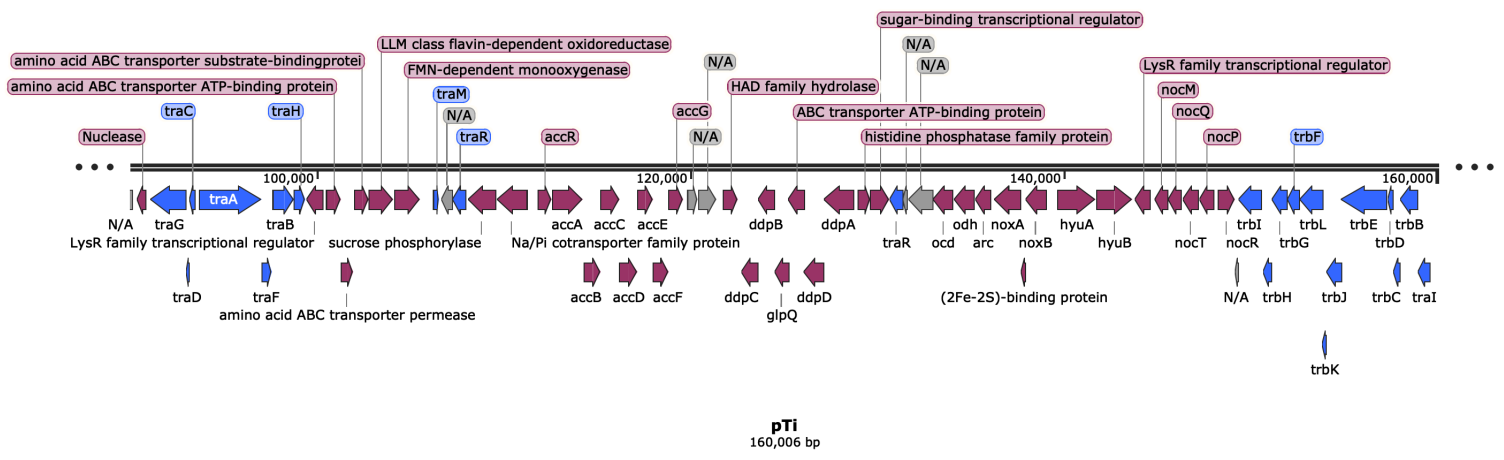

Figure 1: Example schematic of the gene organization of a bacterial conjugation system. 
bioRxiv preprint doi: https://doi.org/10.1101/2021.04.15.440092; this version posted April 16, 2021. The copyright holder for this preprint (which was not certified by peer review) is the author/funder, who has granted bioRxiv a license to display the preprint in perpetuity. It is made available under aCC-BY-NC 4.0 International license.

Approach 1: Use metagenome database to search for conjugative systems

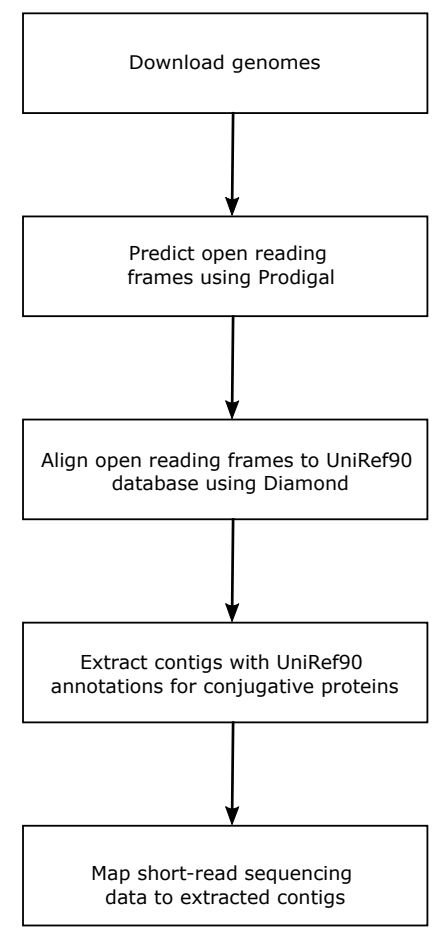

Approach 2: Assemble raw reads and search assemblies for conjugative systems

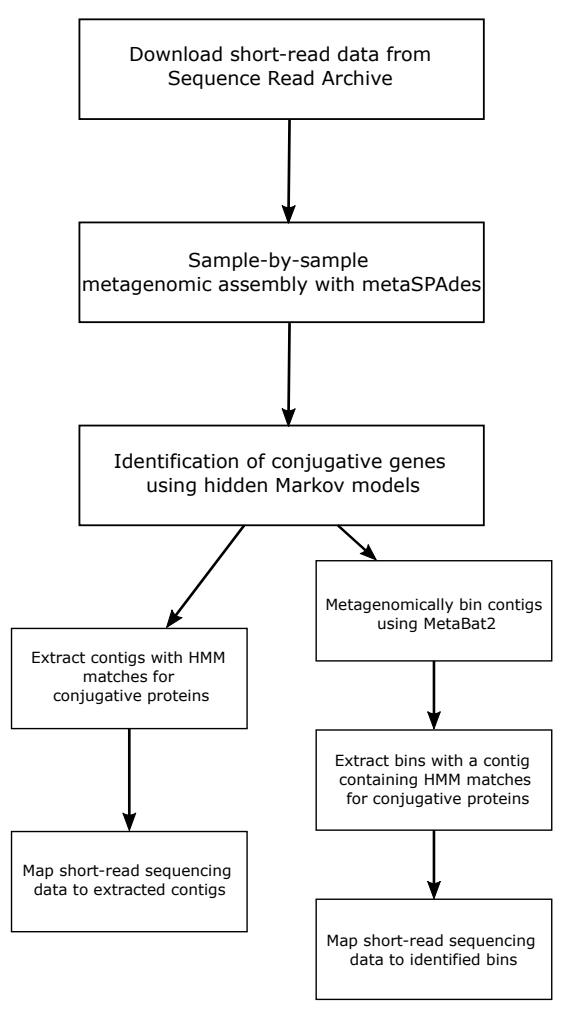

Figure 2: Overview of methods employed in this study. In the left panel is the workflow used to identify conjugative systems from previously assembled human gut bacterial genomes. The right panel outlines the workflow for the assembly of select North American samples and the use of pHMMs to identify the conjugative systems. 


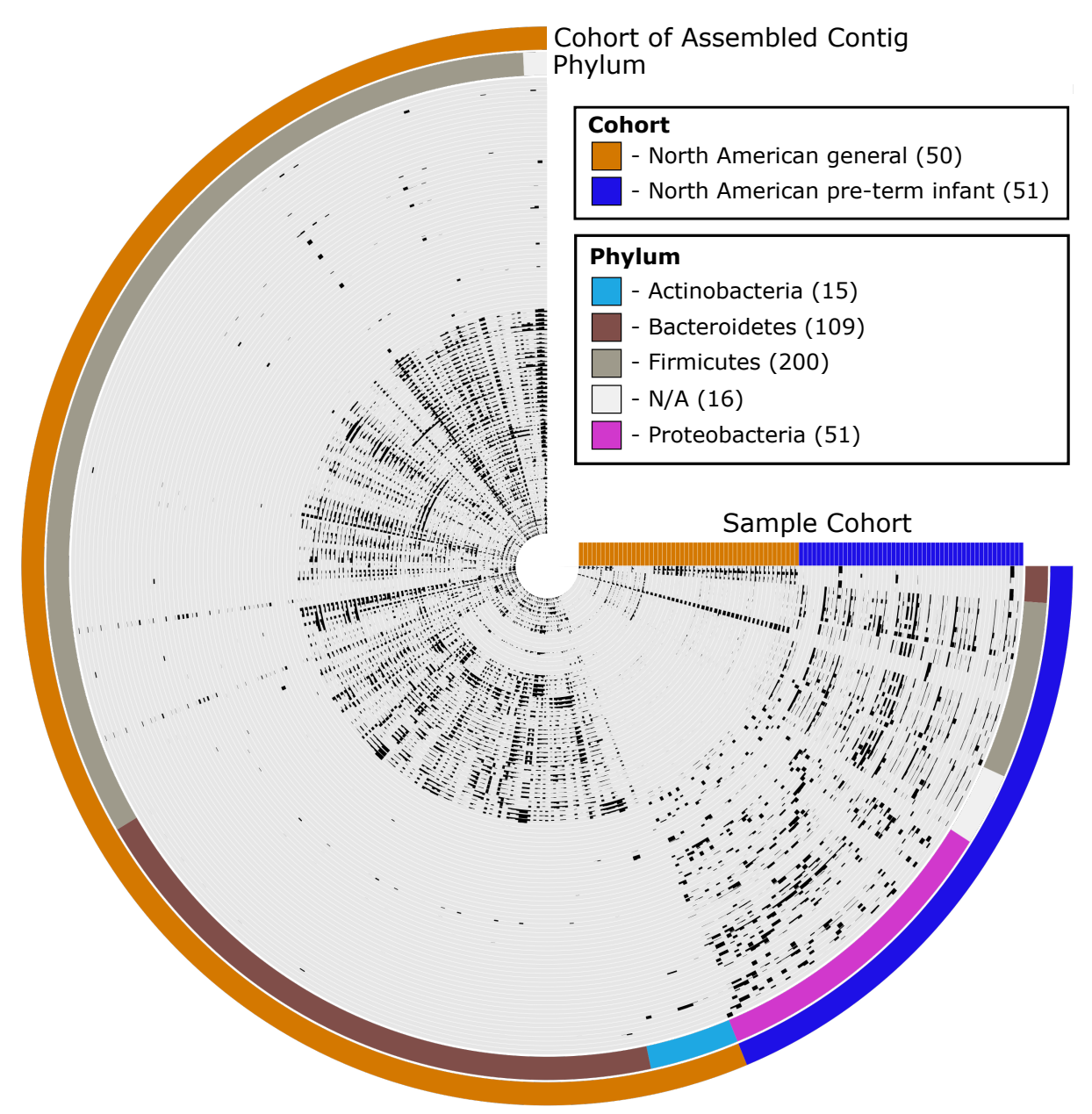

Figure 3: Anvi'o cladogram of potentially conjugative contigs from 51 North American pre-term infants samples and 50 general North American samples. Inner rings of the phylogram represent individual samples, second-most outer ring being the phylum of conjugative system as predicted by Kaiju, and the outermost ring represents the cohort that the conjugative contig was assembled from. Each slice of the circle phylogram are individual conjugative contig identified by pHMMs of conjugative proteins. For the inner plot, intensity of the position represents the mean coverage of the contig for a given sample proportional to the other conjugative systems. 


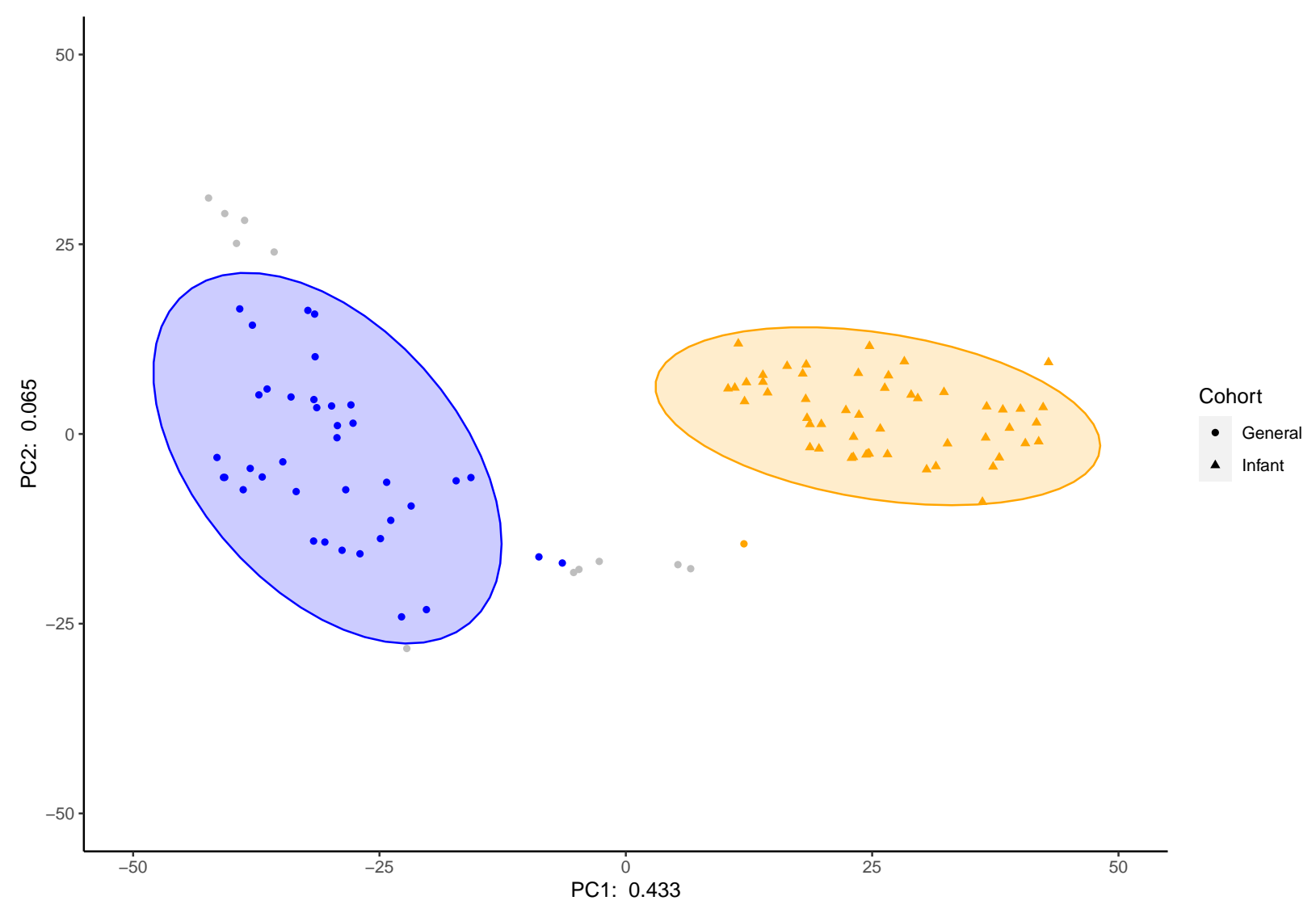

Figure 4: Clustering of the principal component coordinates of the CLR transformed relative abundances of the extracted conjugative regions from the assemblies of North American datasets. Ellipses represent a 95 percent confidence interval using a multivariate t-distribution. 


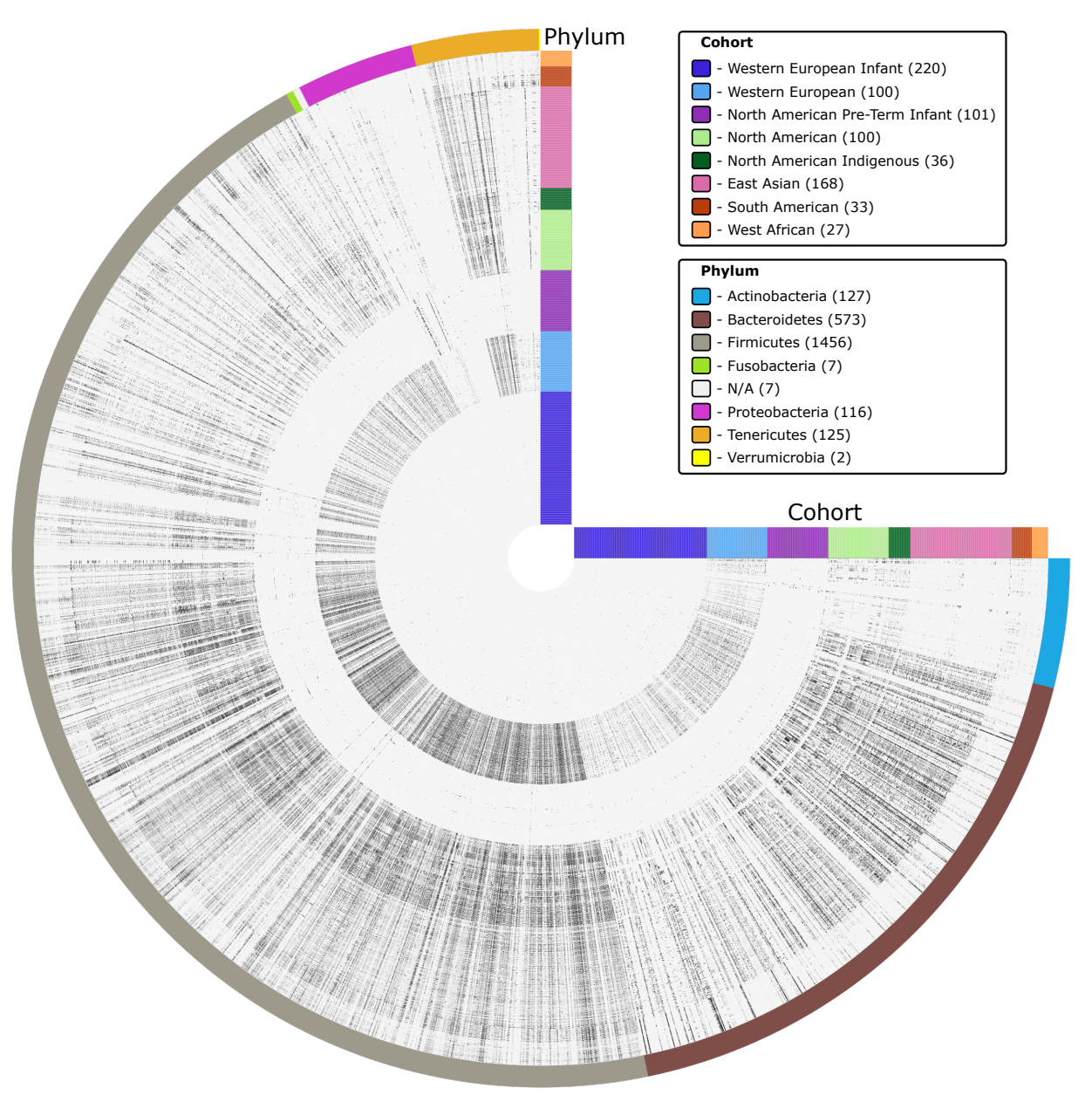

Figure 5: Anvi'o cladogram of potentially conjugative systems originating from 785 samples across 8 cohorts. Inner rings of the phylogram represent individual samples and the outermost ring being the phylum of conjugative system. Each slice of the circle phylogram are individual conjugative regions. For each point on the inner plot, the intensity of the black colouring represents the mean coverage of the system for a given sample proportional to the other conjugative systems. 


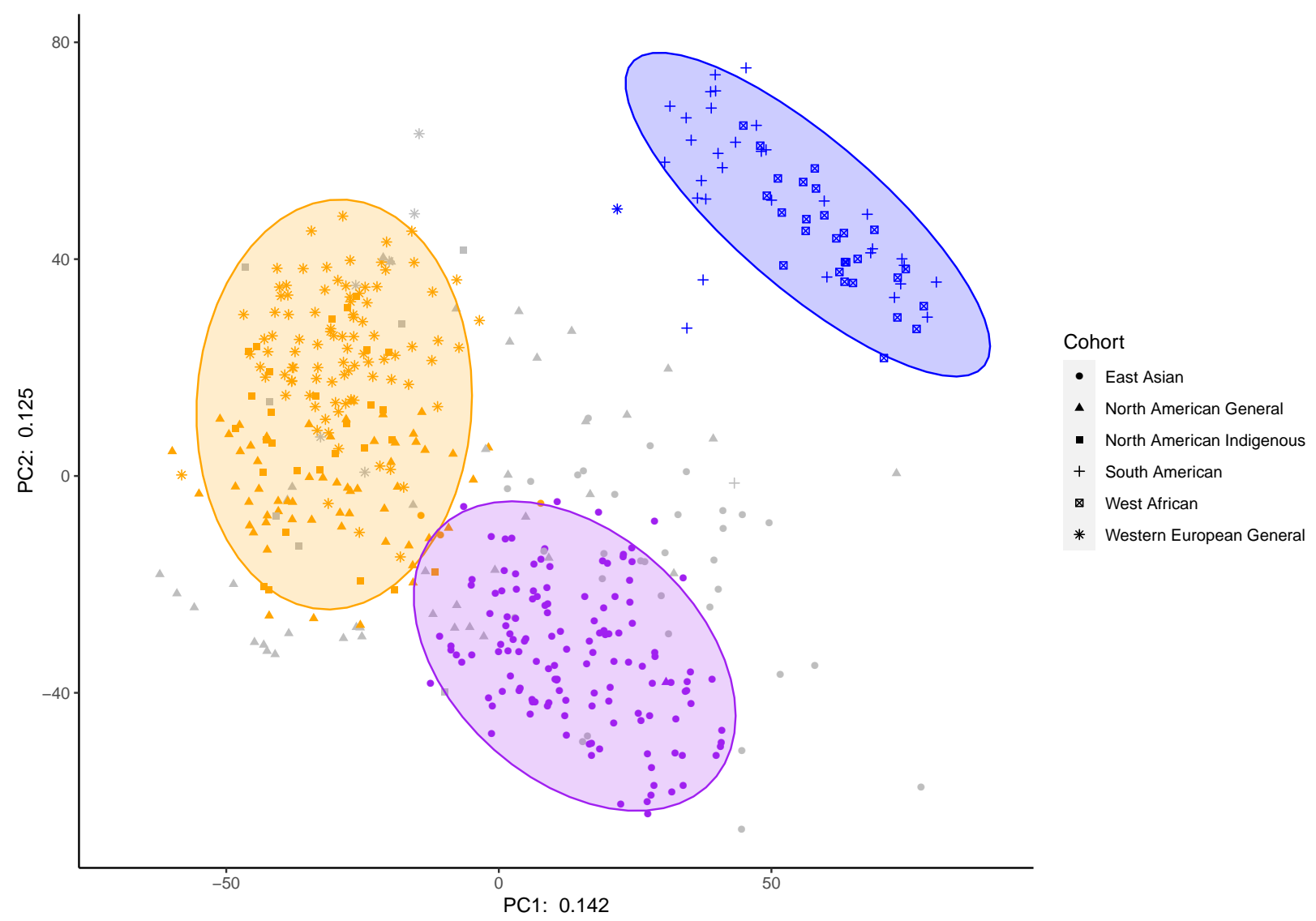

Figure 6: Clustering of the principal component coordinates of the CLR transformed relative abundances of the extracted conjugative regions from the genome database. Ellipses represent a 95 percent confidence interval using a multivariate t-distribution. 
bioRxiv preprint doi: https://doi.org/10.1101/2021.04.15.440092; this version posted April 16, 2021. The copyright holder for this preprint (which was not certified by peer review) is the author/funder, who has granted bioRxiv a license to display the preprint in perpetuity. It is made available under aCC-BY-NC 4.0 International license.

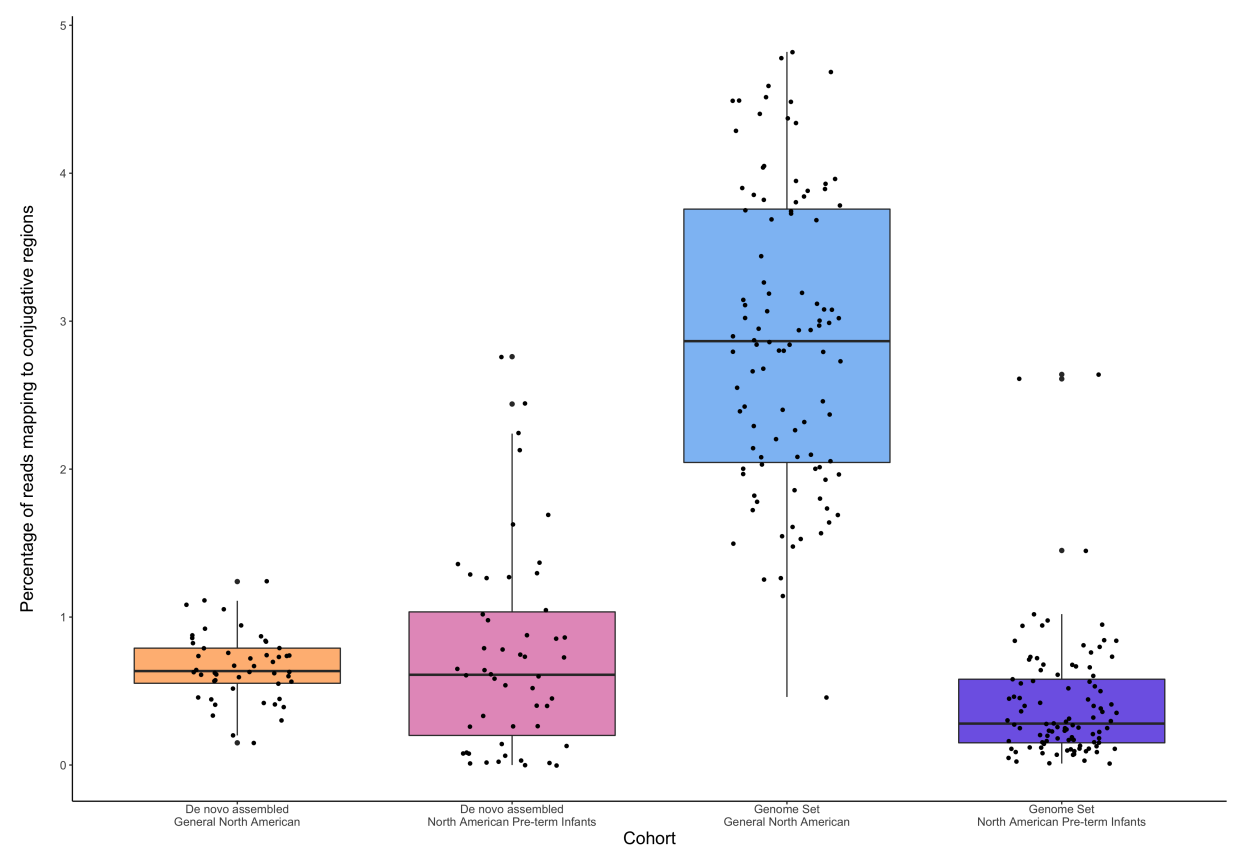

Figure 7: Plot of the percentage of total reads from North American datasets mapping to conjugative regions extracted from de novo assemblies and the genome set, separated by cohort.

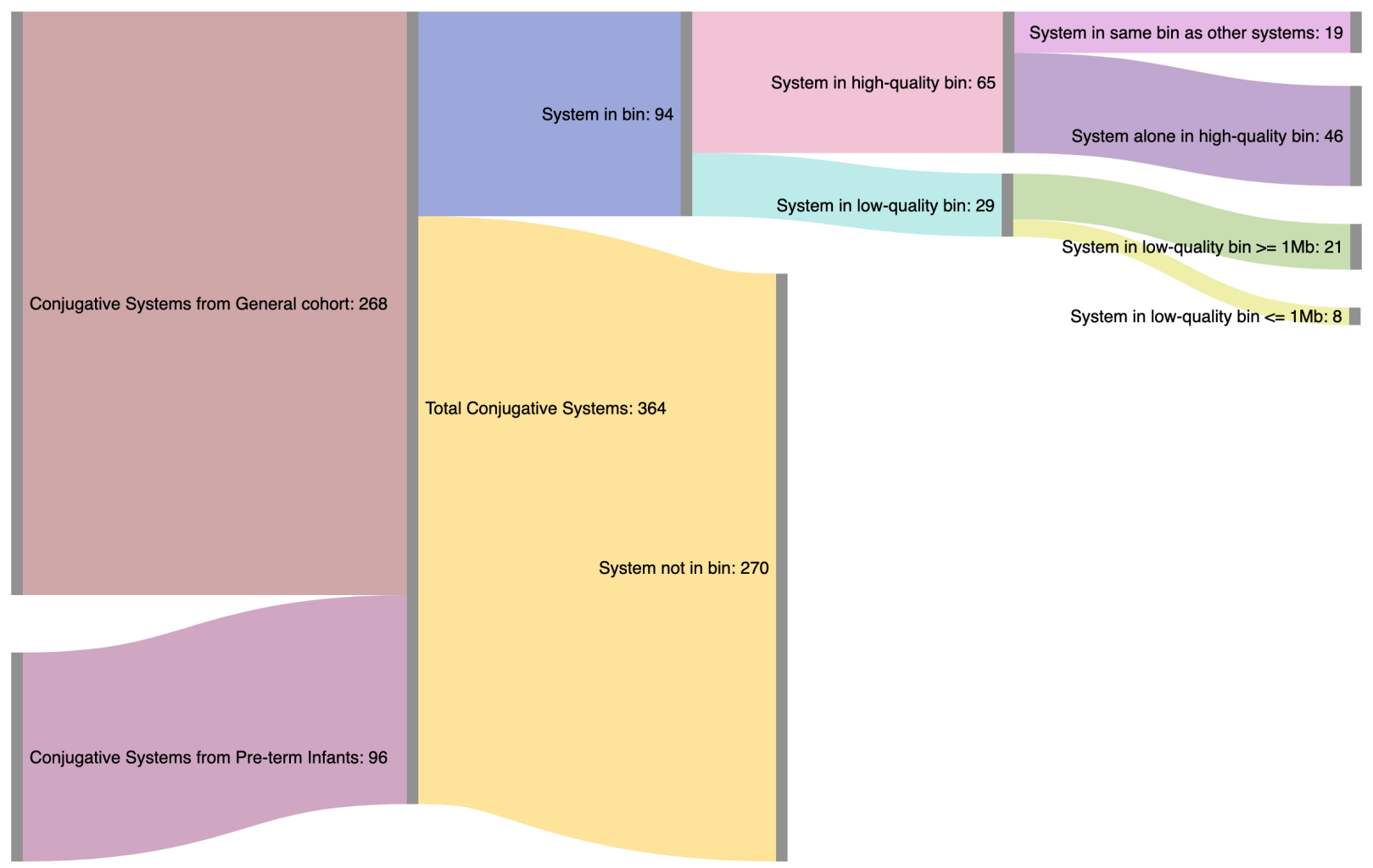

Figure 8: Sankey diagram representing the flow of 364 contigs containing conjugative systems into bins generated by MetaBAT2 from assembled data. 
Supplemental Table 1: Accession numbers and cohorts for samples used in de novo assembly workflow.

Supplemental Table 2: Accession numbers and cohorts for samples used in reference human gut metagenome set workflows.

Supplemental Table 3: Summary data for all predicted conjugative systems.

\section{Declarations}

\section{Ethics approval and consent to participate}

Not applicable.

\section{Consent for publication}

Not applicable.

\section{Availability of data and materials}

All code needed to reproduce the results are available on Github, https://github.com/bjoris33/ humanGutConj_mSystems

\section{Competing interests}

The authors declare that they have no competing interests.

\section{Funding}

Supported by CIHR Project Grant (PJT-159708) to D.R.E. and G.B.G. T.A.H. was supported by an NSERC PGS-D scholarship. B.R.J was supported by the Schulich School of Medicine Dean's Research Scholarship.

\section{Authors' Contributions}

BRJ designed the experiments, analyzed and interpreted the data, and wrote the manuscript. TSB analyzed the data. TAH interpreted the data. DRE designed the experiments, interpreted the data, and provided funding. GBG designed the experiments, interpreted the data, edited the manuscript, and provided funding. 


\section{Acknowledgements}

We thank Daniel Giguere for his input on the analyses and figures.

\section{References}

1. Smillie C, Garcillán-Barcia MP, Francia MV, Rocha EPC, Cruz F de la. Mobility of plasmids. Microbiol Mol Biol Rev. 2010;74:434-52.

2. Daccord A, Ceccarelli D, Burrus V. Integrating conjugative elements of the sxt/r391 family trigger the excision and drive the mobilization of a new class of vibrio genomic islands. Mol Microbiol. 2010;78:576-88.

3. Jiang X, Hall AB, Xavier RJ, Alm EJ. Comprehensive analysis of chromosomal mobile genetic elements in the gut microbiome reveals phylum-level niche-adaptive gene pools. PLoS One. 2019;14:e0223680.

4. Neil K, Allard N, Grenier F, Burrus V, Rodrigue S. Highly efficient gene transfer in the mouse gut microbiota is enabled by the incl2 conjugative plasmid tp114. Commun Biol. 2020;3:523.

5. Hamilton TA, Pellegrino GM, Therrien JA, Ham DT, Bartlett PC, Karas BJ, et al. Efficient inter-species conjugative transfer of a crispr nuclease for targeted bacterial killing. Nat Commun. 2019;10:4544.

6. Peters JM, Koo B-M, Patino R, Heussler GE, Hearne CC, Qu J, et al. Enabling genetic analysis of diverse bacteria with mobile-crispri. Nat Microbiol. 2019;4:244-50.

7. Citorik RJ, Mimee M, Lu TK. Sequence-specific antimicrobials using efficiently delivered rna-guided nucleases. Nat Biotechnol. 2014;32:1141-5.

8. Bikard D, Euler CW, Jiang W, Nussenzweig PM, Goldberg GW, Duportet X, et al. Exploiting crispr-cas nucleases to produce sequence-specific antimicrobials. Nat Biotechnol. 2014;32:1146-50.

9. Gomaa AA, Klumpe HE, Luo ML, Selle K, Barrangou R, Beisel CL. Programmable removal of bacterial strains by use of genome-targeting crispr-cas systems. mBio. 2014;5:e00928-13.

10. Francia MV, Varsaki A, Garcillán-Barcia MP, Latorre A, Drainas C, Cruz F de la. A classification scheme for mobilization regions of bacterial plasmids. FEMS Microbiol Rev. 2004;28:79-100.

11. Nash RP, Habibi S, Cheng Y, Lujan SA, Redinbo MR. The mechanism and control of DNA transfer by the conjugative relaxase of resistance plasmid pCU1. Nucleic Acids Res. 2010;38:5929-43.

12. Becker EC, Meyer RJ. Recognition of oriT for DNA processing at termination of a round of conjugal transfer. J Mol Biol. 2000;300:1067-77.

13. Fronzes R, Christie PJ, Waksman G. The structural biology of type IV secretion systems. Nat Rev Microbiol. 2009;7:703-14. 
14. Cabezón E, Ripoll-Rozada J, Peña A, Cruz F de la, Arechaga I. Towards an integrated model of bacterial conjugation. FEMS Microbiol Rev. 2015;39:81-95.

15. Bhatty M, Laverde Gomez JA, Christie PJ. The expanding bacterial type IV secretion lexicon. Res Microbiol. 164:620-39.

16. Guglielmini J, Cruz F de la, Rocha EPC. Evolution of conjugation and type IV secretion systems. Mol Biol Evol. 2013;30:315-31.

17. Guglielmini J, Quintais L, Garcillán-Barcia MP, Cruz F de la, Rocha EPC. The repertoire of ICE in prokaryotes underscores the unity, diversity, and ubiquity of conjugation. PLoS Genet. 2011;7:e1002222.

18. Shterzer N, Mizrahi I. The animal gut as a melting pot for horizontal gene transfer. Can J Microbiol. $2015 ; 61: 603-5$.

19. Kaufman JH, Terrizzano I, Nayar G, Seabolt E, Agarwal A, Slizovskiy IB, et al. Integrative and conjugative elements (ice) and associated cargo genes within and across hundreds of bacterial genera. bioRxiv. 2020. doi:10.1101/2020.04.07.030320.

20. Arredondo-Alonso S, Willems RJ, Schaik W van, Schürch AC. On the (im)possibility of reconstructing plasmids from whole-genome short-read sequencing data. Microb Genom. 2017;3:e000128.

21. Almeida A, Mitchell AL, Boland M, Forster SC, Gloor GB, Tarkowska A, et al. A new genomic blueprint of the human gut microbiota. Nature. 2019;568:499-504.

22. Krawczyk PS, Lipinski L, Dziembowski A. PlasFlow: Predicting plasmid sequences in metagenomic data using genome signatures. Nucleic Acids Res. 2018;46:e35.

23. Maguire F, Jia B, Gray KL, Lau WYV, Beiko RG, Brinkman FSL. Metagenome-assembled genome binning methods with short reads disproportionately fail for plasmids and genomic islands. Microb Genom. $2020 ; 6$.

24. Suzek BE, Wang Y, Huang H, McGarvey PB, Wu CH, UniProt Consortium. UniRef clusters: A comprehensive and scalable alternative for improving sequence similarity searches. Bioinformatics. 2015;31:926-32. 25. Bushnell B, Rood J, Singer E. BBMerge - accurate paired shotgun read merging via overlap. PLoS One. 2017;12:e0185056.

26. Bolger AM, Lohse M, Usadel B. Trimmomatic: A flexible trimmer for illumina sequence data. Bioinformatics. 2014;30:2114-20.

27. Nurk S, Meleshko D, Korobeynikov A, Pevzner PA. MetaSPAdes: A new versatile metagenomic assembler. Genome Res. 2017;27:824-34.

28. Eren AM, Esen ÖC, Quince C, Vineis JH, Morrison HG, Sogin ML, et al. Anvi'o: An advanced analysis and visualization platform for 'omics data. PeerJ. 2015;3:e1319. 
29. Eddy SR. Accelerated profile HMM searches. PLoS Comput Biol. 2011;7:e1002195.

30. Hyatt D, Chen G-L, Locascio PF, Land ML, Larimer FW, Hauser LJ. Prodigal: Prokaryotic gene recognition and translation initiation site identification. BMC Bioinformatics. 2010;11:119.

31. Langmead B, Salzberg SL. Fast gapped-read alignment with Bowtie 2. Nat Methods. 2012;9:357-9.

32. Menzel P, Ng KL, Krogh A. Fast and sensitive taxonomic classification for metagenomics with Kaiju. Nat Commun. 2016;7:11257.

33. Robertson J, Bessonov K, Schonfeld J, Nash JHE. Universal whole-sequence-based plasmid typing and its utility to prediction of host range and epidemiological surveillance. Microb Genom. 2020;6.

34. McInnes L, Healy J, Astels S. hdbscan: Hierarchical density based clustering. Journal of Open Source Software. 2017;2:205. doi:10.21105/joss.00205.

35. Kang DD, Li F, Kirton E, Thomas A, Egan R, An H, et al. MetaBAT 2: An adaptive binning algorithm for robust and efficient genome reconstruction from metagenome assemblies. PeerJ. 2019;7:e7359.

36. Parks DH, Imelfort M, Skennerton CT, Hugenholtz P, Tyson GW. CheckM: Assessing the quality of microbial genomes recovered from isolates, single cells, and metagenomes. Genome Res. 2015;25:1043-55.

37. Olm MR, Brown CT, Brooks B, Banfield JF. DRep: A tool for fast and accurate genomic comparisons that enables improved genome recovery from metagenomes through de-replication. ISME J. 2017;11:2864-8. 38. Buchfink B, Xie C, Huson DH. Fast and sensitive protein alignment using DIAMOND. Nat Methods. $2015 ; 12: 59-60$.

39. Li H, Handsaker B, Wysoker A, Fennell T, Ruan J, Homer N, et al. The sequence alignment/map format and SAMtools. Bioinformatics. 2009;25:2078-9.

40. Pasolli E, Asnicar F, Manara S, Zolfo M, Karcher N, Armanini F, et al. Extensive unexplored human microbiome diversity revealed by over 150,000 genomes from metagenomes spanning age, geography, and lifestyle. Cell. 2019;176:649-662.e20.

41. Milani C, Duranti S, Bottacini F, Casey E, Turroni F, Mahony J, et al. The first microbial colonizers of the human gut: Composition, activities, and health implications of the infant gut microbiota. Microbiol Mol Biol Rev. 2017;81.

42. Gibson MK, Wang B, Ahmadi S, Burnham C-AD, Tarr PI, Warner BB, et al. Developmental dynamics of the preterm infant gut microbiota and antibiotic resistome. Nat Microbiol. 2016;1:16024.

43. Moss EL, Maghini DG, Bhatt AS. Complete, closed bacterial genomes from microbiomes using nanopore sequencing. Nature Biotechnology. 2020. doi:10.1038/s41587-020-0422-6.

44. Giguere DJ, Bahcheli AT, Joris BR, Paulssen JM, Gieg LM, Flatley MW, et al. Complete and validated genomes from a metagenome. bioRxiv. 2020. doi:10.1101/2020.04.08.032540. 
bioRxiv preprint doi: https://doi.org/10.1101/2021.04.15.440092; this version posted April 16, 2021. The copyright holder for this preprint (which

was not certified by peer review) is the author/funder, who has granted bioRxiv a license to display the preprint in perpetuity. It is made available under aCC-BY-NC 4.0 International license.

45. Beaulaurier J, Zhu S, Deikus G, Mogno I, Zhang X-S, Davis-Richardson A, et al. Metagenomic binning and association of plasmids with bacterial host genomes using dna methylation. Nat Biotechnol. 2018;36:619. 


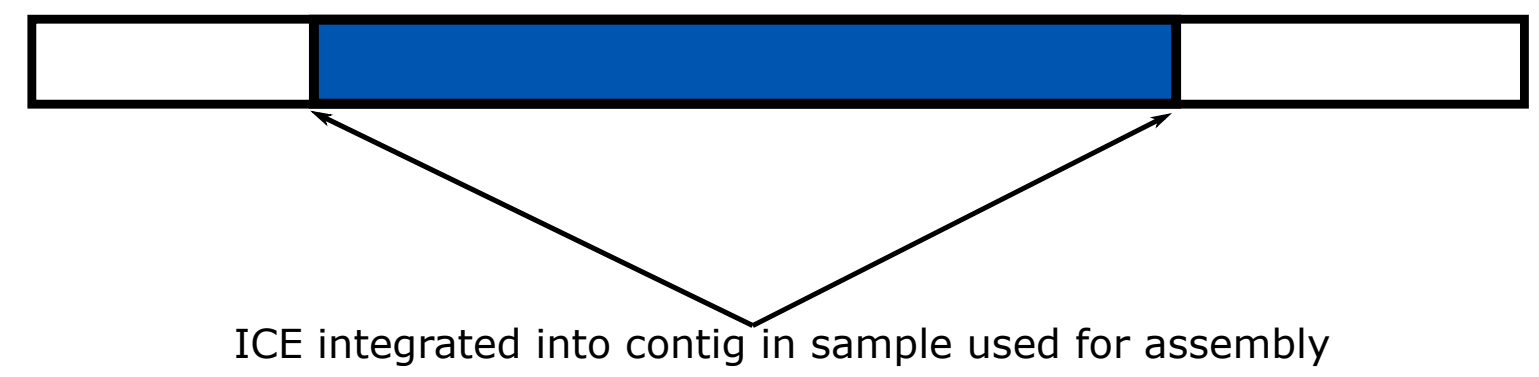

Mapping coverage in sample containing ICE:

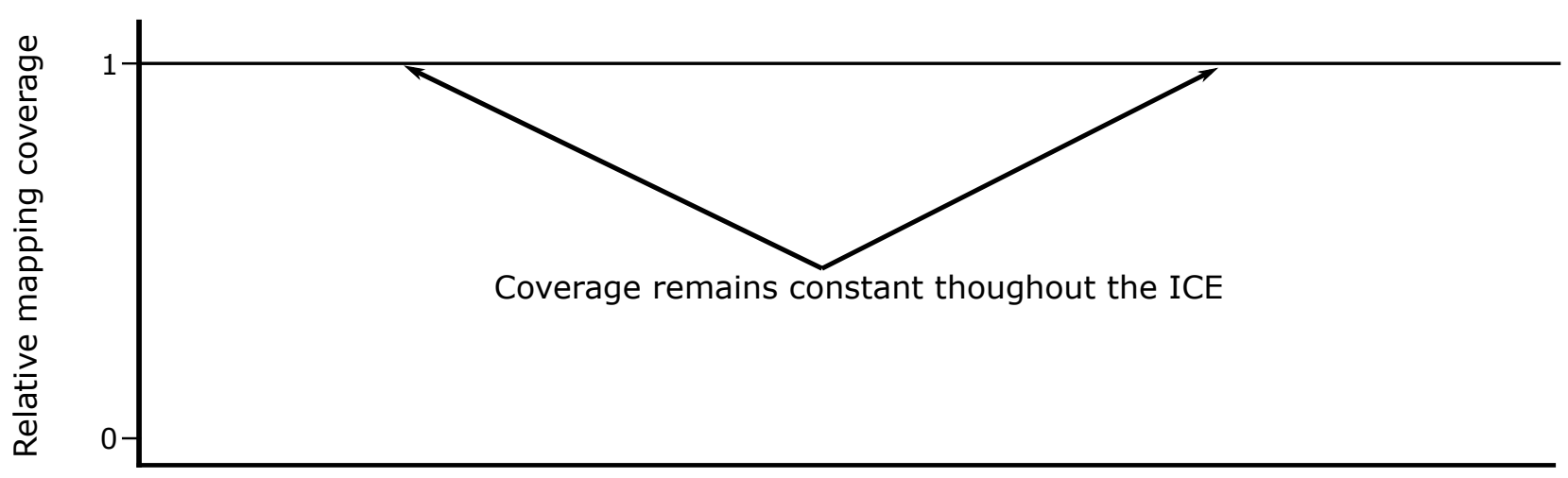

Position on contig

\section{Mapping coverage in sample lacking ICE:}

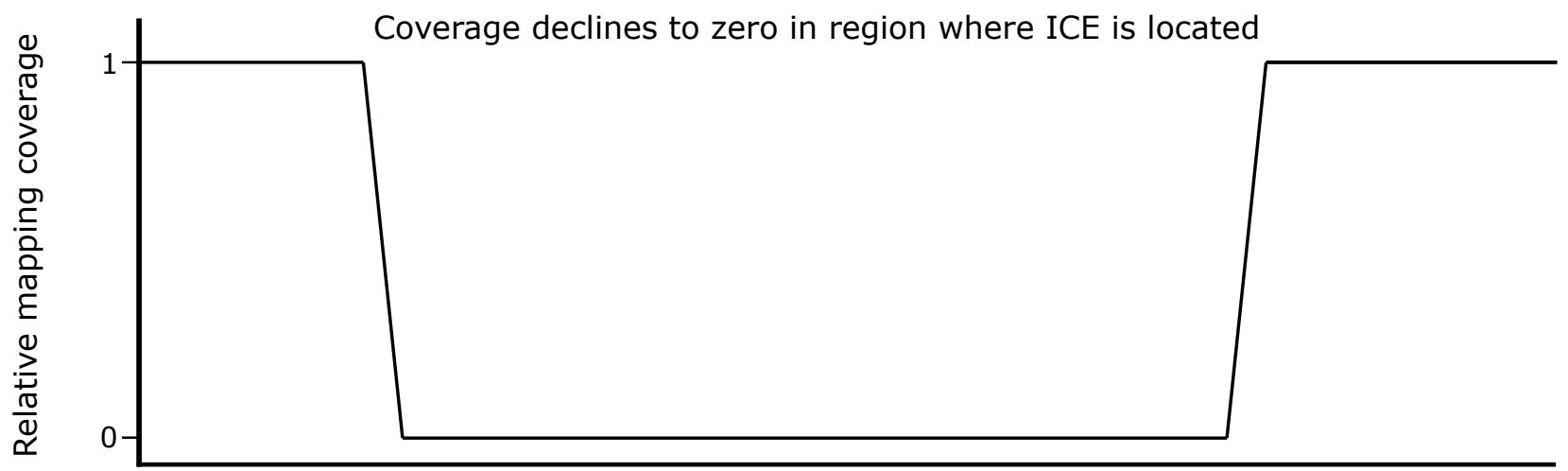

\section{Position on contig}

Figure 1: Conceptual diagram of the mapping coverage of an assembled integrative and conjugative element. The mapping coverage in the first plot shows an even mapping coverage across the contig because the ICE is present in the sample and the average mapping coverage of the contig would be an accurate metric. In the second plot, the ICE is missing in the sample and the mapping coverage falls to zero where the ICE is located on the contig. As a way to quantify the presence of the ICE, the average mapping coverage for the entire contig would be artificially high. Limiting the mapping to only the region containing the conjugative proteins solves this issue. 
bioRxiv preprint doi: https://doi.org/10.1101/2021.04.15.440092; this version posted April 16, 2021. The copyright holder for this preprint (which

was not certified by peer review) is the author/funder, who has granted bioRxiv a license to display the preprint in perpetuity. It is made available under aCC-BY-NC 4.0 International license.

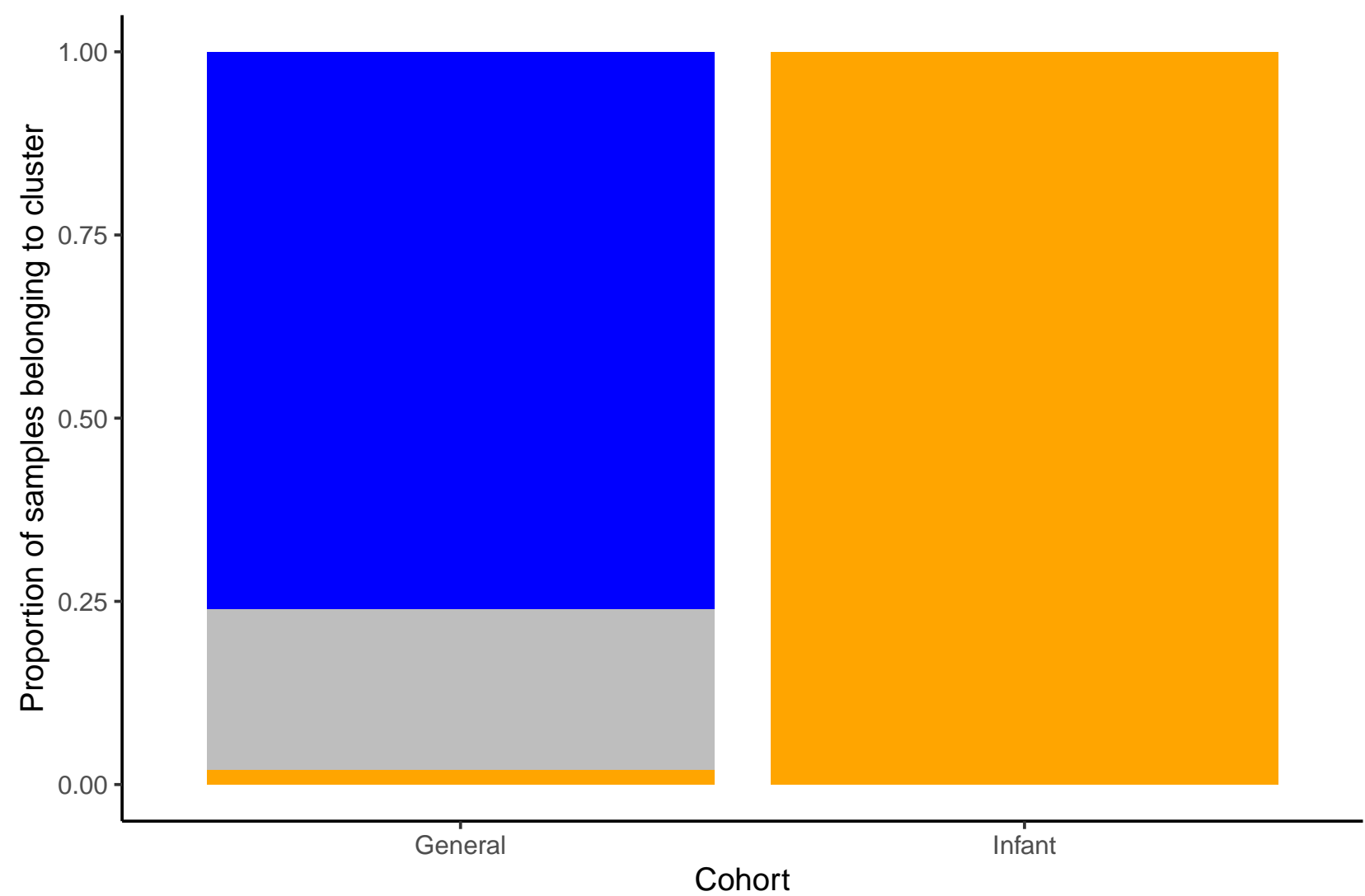

Figure 2: Stacked bar plot of the proportions of samples belonging to the hdbscan clusters from each cohort. 
bioRxiv preprint doi: https://doi.org/10.1101/2021.04.15.440092; this version posted April 16, 2021. The copyright holder for this preprint (which

was not certified by peer review) is the author/funder, who has granted bioRxiv a license to display the preprint in perpetuity. It is made available under aCC-BY-NC 4.0 International license.

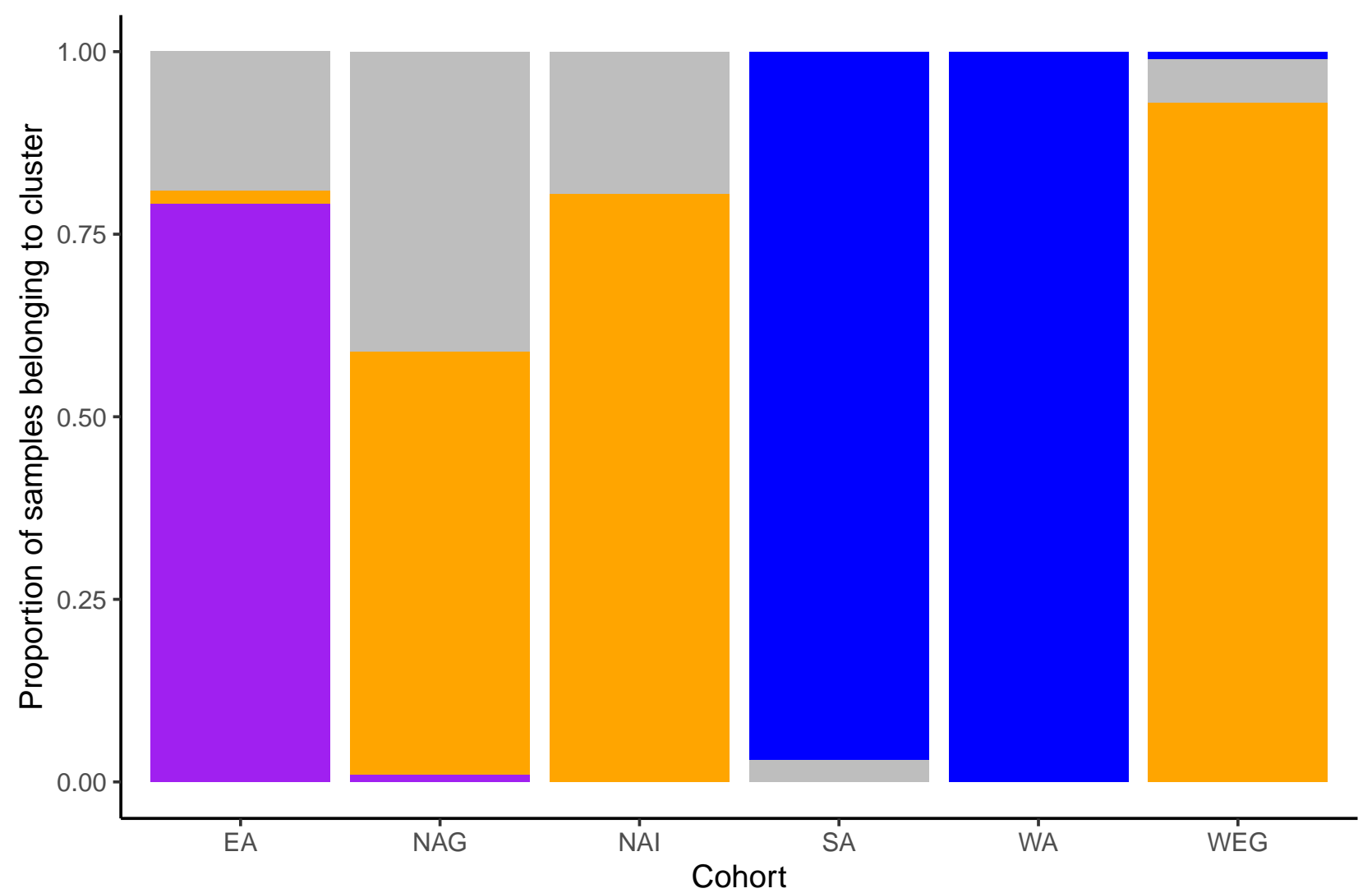

Figure 3: Stacked bar plot of the proportions of samples belonging to the hdbscan clusters from each cohort. 


\begin{tabular}{|c|c|c|}
\hline 131692 & pre-term & infant \\
\hline SRR3131694 & pre-term & infant \\
\hline SRR3131696 & pre-term & infant \\
\hline SRR3131698 & pre-term & infant \\
\hline SRR3131700 & pre-term & infant \\
\hline SRR3131702 & pre-term & infant \\
\hline SRR3131704 & pre-term & infant \\
\hline RR3131706 & pre-term & infant \\
\hline RR3131708 & pre-term & infant \\
\hline RR3131710 & pre-term & \\
\hline R3131712 & pre-term & \\
\hline R3131714 & pre-term & infant \\
\hline R3131716 & pre-term & infant \\
\hline R3131718 & pre-term & infant \\
\hline R3131720 & pre-term & infant \\
\hline RR3131722 & pre-term & infant \\
\hline RR3131724 & pre-term & infant \\
\hline RR3131726 & pre-term & infant \\
\hline RR3131728 & pre-term & infant \\
\hline RR3131730 & pre-term & infant \\
\hline RR3131732 & pre-term & infant \\
\hline R3131734 & pre-term & infant \\
\hline R3131736 & pre-term & infant \\
\hline R3131738 & pre-term & infant \\
\hline R3131740 & pre-term & infant \\
\hline R3131742 & pre-term & infant \\
\hline R3131744 & pre-term & infant \\
\hline R3131746 & pre-term & infant \\
\hline R3131748 & pre-term & infant \\
\hline R3131750 & pre-term & infant \\
\hline R3131752 & pre-term & infant \\
\hline 23131754 & pre-term & infant \\
\hline R3131756 & pre-term & infant \\
\hline 23131758 & pre-term & infant \\
\hline R3131760 & pre-term & infant \\
\hline R3131762 & pre-term & infant \\
\hline R3131764 & pre-term & infant \\
\hline R3131766 & pre-term & infant \\
\hline R3131768 & pre-term & infant \\
\hline 3131770 & pre-term & infant \\
\hline 3131772 & pre-term & infant \\
\hline R3131774 & pre-term & infant \\
\hline 3131776 & pre-term & infant \\
\hline 3131778 & pre-term & $\inf$ \\
\hline R3131780 & pre-term & infant \\
\hline R3131782 & pre-term & infant \\
\hline R3131784 & pre-term & infant \\
\hline R3131786 & pre-term & infant \\
\hline RR3131788 & pre-term & infant \\
\hline R3131790 & pre-term & infant \\
\hline R3131812 & pre-term & infant \\
\hline 25650110 & general & \\
\hline R5650111 & general & \\
\hline 5650112 & general & \\
\hline
\end{tabular}


bioRxiv preprint doi: https://doi.org/10.1101/2021.04.15.440092; this version posted April 16, 2021. The copyright holder for this preprint (which was not certified by peer review) is the author/funder, who has granted bioRxiv a license to display the preprint in perpetuity. It is made available under aCC-BY-NC 4.0 International license.

\begin{tabular}{|c|c|}
\hline SRR5 650113 & \\
\hline SRR5 650114 & genera \\
\hline SRR5 650115 & gener \\
\hline R5650116 & 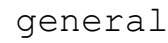 \\
\hline R56501. & gener \\
\hline R56501. & ne \\
\hline 6501 & ne \\
\hline 6501 & \\
\hline R565012 & $g e$ \\
\hline 501 & \\
\hline 65012 & ner \\
\hline 65012 & gene \\
\hline 565012 & ne. \\
\hline 565012 & ene \\
\hline 565012 & $n e$ \\
\hline 256501 & ener \\
\hline 256501 & ner \\
\hline 56501 & ener \\
\hline 56501 & nes \\
\hline 56501 & ne \\
\hline 56501 & ne. \\
\hline 2565013 & enes \\
\hline 56501 & ener \\
\hline 56501 & ener \\
\hline 256501 & ener \\
\hline 256501 & ener \\
\hline 56501 & ene: \\
\hline 256501 & ene: \\
\hline 56501 & ene: \\
\hline 56501 & ener \\
\hline 56501 & gener \\
\hline 25650144 & gener \\
\hline 25650145 & gener \\
\hline 5650146 & gener \\
\hline 25650147 & gener \\
\hline 5650148 & ener \\
\hline 5650149 & gener \\
\hline 5650150 & ener \\
\hline 5650151 & gener \\
\hline 5650152 & gener \\
\hline 5650153 & ner \\
\hline 5650154 & ner \\
\hline 5650155 & ne: \\
\hline 565015 & ne \\
\hline 6501 & he \\
\hline 501 & ene \\
\hline 6501 & 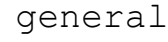 \\
\hline
\end{tabular}


bioRxiv preprint doi: https://doi.org/10.1101/2021.04.15.440092; this version posted April 16, 2021. The copyright holder for this preprint (which was not certified by peer review) is the author/funder, who has granted bioRxiv a license to display the preprint in perpetuity. It is made available under aCC-BY-NC 4.0 International license.

\begin{tabular}{|c|c|c|}
\hline 60132 & Western & European \\
\hline ERR260133 & Western & European \\
\hline ERR2 60134 & Western & European \\
\hline ERR2 60135 & Western & European \\
\hline ERR260136 & Western & European \\
\hline ERR260137 & Western & European \\
\hline ERR2 60138 & Western & European \\
\hline RR260139 & Western & European \\
\hline RR2 60140 & Western & European \\
\hline RR2601 & Western & European \\
\hline RR260142 & Western & European \\
\hline RR260143 & Western & European \\
\hline RR2 60144 & Western & European \\
\hline RR260145 & Western & European \\
\hline RR260146 & Western & European \\
\hline RR2 60147 & Western & European \\
\hline RRR2 60148 & Western & European \\
\hline RR260149 & Western & European \\
\hline RR2 60150 & Western & European \\
\hline RR260151 & Western & European \\
\hline RR2 60152 & Western & European \\
\hline RRR260153 & Western & European \\
\hline ERR260154 & Western & European \\
\hline RRR260155 & Western & European \\
\hline PRR260156 & Western & European \\
\hline RRR260157 & Western & European \\
\hline ERR260158 & Western & European \\
\hline ERR260159 & Western & European \\
\hline ERR260160 & Western & European \\
\hline RR2 60161 & Western & European \\
\hline ERR260162 & Western & European \\
\hline PRR260163 & Western & European \\
\hline ERR260164 & Western & European \\
\hline ERR2 6016: & Western & European \\
\hline ERR260166 & Western & European \\
\hline ERR2 60167 & Western & European \\
\hline ERR260168 & Western & European \\
\hline ERR2 60169 & Western & European \\
\hline ERR2 60170 & Western & European \\
\hline ERR2 60171 & Western & European \\
\hline ERR2 60172 & Western & European \\
\hline ERR2 60173 & Western & European \\
\hline ERR2 60174 & Western & European \\
\hline ERR260175 & West & European \\
\hline ERR260176 & Western & European \\
\hline ERR2 60177 & Western & European \\
\hline ERR260178 & Western & European \\
\hline ERR260179 & Western & European \\
\hline ERR2 60180 & Western & European \\
\hline ERR2 60181 & Western & European \\
\hline ERR2 60182 & Western & European \\
\hline ERR260183 & Western & European \\
\hline ERR26018 & Western & Europea \\
\hline ERR26018 & Western & Europea \\
\hline
\end{tabular}


bioRxiv preprint doi: https://doi.org/10.1101/2021.04.15.440092; this version posted April 16, 2021. The copyright holder for this preprint (which was not certified by peer review) is the author/funder, who has granted bioRxiv a license to display the preprint in perpetuity. It is made available under aCC-BY-NC 4.0 International license.

\begin{tabular}{|c|c|}
\hline ERR2 60186 & Western European \\
\hline RR2 60187 & Western European \\
\hline RR260188 & Western European \\
\hline RR260189 & Western European \\
\hline RR2 60190 & Western European \\
\hline ERR2 60191 & Western European \\
\hline ERR2 60192 & Western European \\
\hline ERR2 60193 & Western European \\
\hline ERR260194 & Western European \\
\hline ERR260195 & Western European \\
\hline RR260196 & Western European \\
\hline RR260197 & Western European \\
\hline RR2 601 & Western European \\
\hline RR2601 & Western European \\
\hline RR2 60200 & Western European \\
\hline RR260201 & Western European \\
\hline RR2 60202 & Western European \\
\hline RR2 60203 & Western European \\
\hline RR260204 & Western European \\
\hline RR260205 & Western European \\
\hline RR260206 & Western European \\
\hline RR260207 & Western European \\
\hline RR260208 & Western European \\
\hline RR260209 & Western European \\
\hline RR260210 & Western European \\
\hline RR260211 & Western European \\
\hline RR260214 & Western European \\
\hline RR260215 & Western European \\
\hline RR260216 & Western European \\
\hline RR260217 & Western European \\
\hline RR2 60218 & Western European \\
\hline RR2 60219 & Western European \\
\hline RR2 60220 & Western European \\
\hline RR260221 & Western European \\
\hline RR260222 & Western European \\
\hline RR2 60223 & Western European \\
\hline RR260224 & Western European \\
\hline RR2 60225 & Western European \\
\hline RR2 60226 & Western European \\
\hline RR2 $6022^{\circ}$ & Western European \\
\hline RR26022 & Western European \\
\hline R26022 & Western European \\
\hline ERR26023 & Western European \\
\hline ERR260231 & Western European \\
\hline ERR260232 & Western European \\
\hline ERR260233 & Western European \\
\hline SRR1761664 & South American \\
\hline SRR1761665 & South American \\
\hline SRR1761666 & South American \\
\hline SRR1761667 & South American \\
\hline SRR1761668 & South American \\
\hline SRR1761669 & South American \\
\hline SRR1761670 & South American \\
\hline SRR1 761671 & South American \\
\hline
\end{tabular}


SRR2912775 North American Indigenous SRR2912776 North American Indigenous SRR2912777 North American Indigenous SRR2912778 North American Indigenous SRR2912779 North American Indigenous SRR2912780 North American Indigenous SRR2912781 North American Indigenous SRR2912782 North American Indigenous SRR2912783 North American Indigenous SRR2 912784 North American Indigenous SRR2912785 North American Indigenous SRR2912786 North American Indigenous SRR2912787 North American Indigenous SRR2912788 North American Indigenous SRR2912789 North American Indigenous SRR2912790 North American Indigenous SRR2912791 North American Indigenous SRR2912792 North American Indigenous SRR2912793 North American Indigenous SRR2912794 North American Indigenous SRR2 912795 North American Indigenous SRR2912796 North American Indigenous SRR2912797 North American Indigenous SRR2912798 North American Indigenous SRR2912799 North American Indigenous SRR2912800 North American Indigenous SRR2912801 North American Indigenous SRR2912802 North American Indigenous SRR2912803 North American Indigenous SRR2912804 North American Indigenous SRR2912805 North American Indigenous SRR2912806 North American Indigenous SRR2912807 North American Indigenous SRR2912809 North American Indigenous SRR2912810 North American Indigenous SRR2912811 North American Indigenous SRR3131792 North American Pre-term Infant SRR3131794 North American Pre-term Infant SRR3131796 North American Pre-term Infant SRR3131798 North American Pre-term Infant SRR3131800 North American Pre-term Infant SRR3131802 North American Pre-term Infant SRR3131804 North American Pre-term Infant SRR3131806 North American Pre-term Infant SRR3131808 North American Pre-term Infant SRR3131810 North American Pre-term Infant SRR3131812 North American Pre-term Infant SRR3131814 North American Pre-term Infant SRR3131816 North American Pre-term Infant SRR3131818 North American Pre-term Infant SRR3131820 North American Pre-term Infant SRR3131822 North American Pre-term Infant SRR3131824 North American Pre-term Infant SRR3131826 North American Pre-term Infant 
bioRxiv preprint doi: https://doi.org/10.1101/2021.04.15.440092; this version posted April 16, 2021. The copyright holder for this preprint (which was not certified by peer review) is the author/funder, who has granted bioRxiv a license to display the preprint in perpetuity. It is made available under aCC-BY-NC 4.0 International license.

\begin{tabular}{|c|c|c|}
\hline 02 & st & \\
\hline SRR341603 & East & Asian \\
\hline SRR3 41604 & East & \\
\hline 605 & East & \\
\hline & & \\
\hline & & \\
\hline & & \\
\hline & & \\
\hline & & \\
\hline & & \\
\hline 416 & & \\
\hline 416 & & \\
\hline 416 & & \\
\hline 416 & & \\
\hline 416 & & \\
\hline 116 & ast & \\
\hline 116 & ast & \\
\hline 116 & ast & \\
\hline 416 & st & \\
\hline 116 & st & \\
\hline 16 & & \\
\hline 416 & ast & \\
\hline 3416 & ast & Asi \\
\hline 416 & ast & Asi \\
\hline 416 & st & Asi \\
\hline 416 & st & $\pi$ \\
\hline 116 & & \\
\hline 116 & & \\
\hline 116 & & 10 \\
\hline 316 & st & A \\
\hline 3416 & East & ASlá \\
\hline 3416 & East & ASI \\
\hline 416 & East & $A s$ \\
\hline 416 & st & As \\
\hline 416 & st & $A S$ \\
\hline 416 & st & $A s$ \\
\hline 316 & st & A \\
\hline 416 & st & A \\
\hline 416 & st & A \\
\hline 416 & st & \\
\hline 416 & st & \\
\hline 416 & st & \\
\hline 3416 & st & \\
\hline 341645 & st & As \\
\hline 341646 & East & $A s$ \\
\hline 341647 & East & Asi \\
\hline 341648 & East & Asi \\
\hline 341649 & East & Asi \\
\hline R34165 & East & Asi \\
\hline 23416 & $E \bar{a}$ & Asi \\
\hline 23416 & $\mathrm{E}$ & Asi \\
\hline 3116 & & As \\
\hline & & \\
\hline & & \\
\hline
\end{tabular}


bioRxiv preprint doi: https://doi.org/10.1101/2021.04.15.440092; this version posted April 16, 2021. The copyright holder for this preprint (which was not certified by peer review) is the author/funder, who has granted bioRxiv a license to display the preprint in perpetuity. It is made available under aCC-BY-NC 4.0 International license.

$\begin{array}{ll}\text { SRR341656 } & \text { East Asian } \\ \text { SRR341657 } & \text { East Asian } \\ \text { SRR341658 } & \text { East Asian } \\ \text { SRR341659 } & \text { East Asian } \\ \text { SRR341660 } & \text { East Asian } \\ \text { SRR341661 } & \text { East Asian } \\ \text { SRR341662 } & \text { East Asian } \\ \text { SRR341663 } & \text { East Asian } \\ \text { SRR341664 } & \text { East Asian } \\ \text { SRR341665 } & \text { East Asian } \\ \text { SRR341666 } & \text { East Asian } \\ \text { SRR341667 } & \text { East Asian } \\ \text { SRR341668 } & \text { East Asian } \\ \text { SRR341669 } & \text { East Asian } \\ \text { SRR341670 } & \text { East Asian } \\ \text { SRR341671 } & \text { East Asian } \\ \text { SRR341672 } & \text { East Asian } \\ \text { SRR341673 } & \text { East Asian } \\ \text { SRR341674 } & \text { East Asian } \\ \text { SRR341675 } & \text { East Asian } \\ \text { SRR341676 } & \text { East Asian } \\ \text { SRR341677 } & \text { East Asian } \\ \text { SRR341678 } & \text { East Asian } \\ \text { SRR341679 } & \text { East Asian } \\ \text { SRRR3441680 } & \text { East Asian } \\ \text { SRR34171705 } & \text { East Asian } \\ \text { SRR3417106 } & \text { East Asian } \\ \text { SRR341681 } & \text { East Asian } \\ \text { SRRR341708 } & \text { East Asian } \\ \text { SRR31682 } & \text { East Asian } \\ \text { SRR341683 } & \text { East Asian } \\ \text { SRR341684 } & \text { East Asian } \\ \text { SRR341685 } & \text { East Asian } \\ \text { SRR341686 } & \text { East Asian } \\ \text { SRR341687 } & \text { East Asian } \\ \text { SRR341688 } & \text { East Asian } \\ \text { SRR341689 } & \text { East Asian } \\ \text { SRR341690 } & \text { East Asian } \\ \text { SRR341691 } & \text { East Asian } \\ \text { SRR341692 } & \text { East Asian } \\ \text { SRR341693 } & \text { East Asian } \\ \text { SRR341694 } & \text { East Asian } \\ \text { SRR341695 } & \text { East Asian } \\ \text { SRR341696 } & \text { East Asian } \\ \text { SRR341697 } & \text { East Asian } \\ \text { SRR341698 } & \text { East Asian } \\ \text { SRR341699 } & \text { East Asian } \\ \text { Sast Ast Asian } & \text { East Astan } \\ \text { Sast Asian }\end{array}$


bioRxiv preprint doi: https://doi.org/10.1101/2021.04.15.440092; this version posted April 16, 2021. The copyright holder for this preprint (which was not certified by peer review) is the author/funder, who has granted bioRxiv a license to display the preprint in perpetuity. It is made available under aCC-BY-NC 4.0 International license.

\begin{tabular}{|c|c|c|}
\hline SRR3 41710 & East Asian & \\
\hline SRR3 41711 & East Asian & \\
\hline RR341712 & East Asian & \\
\hline SRR3 41713 & East Asian & \\
\hline RR341714 & East Asian & \\
\hline RR341715 & East Asian & \\
\hline SRR341716 & East Asian & \\
\hline RR341717 & East Asian & \\
\hline RR341718 & East Asian & \\
\hline SRR341719 & East Asian & \\
\hline RR341720 & East Asian & \\
\hline RR341721 & East Asian & \\
\hline SRR341722 & East Asian & \\
\hline SRR3 41723 & East Asian & \\
\hline RR341724 & East Asian & \\
\hline RR341725 & East Asian & \\
\hline SRR413615 & East Asian & \\
\hline SRR413626 & East Asian & \\
\hline SRR413631 & East Asian & \\
\hline SRR4 13632 & East Asian & \\
\hline SRR413633 & East Asian & \\
\hline $\operatorname{SRR} 413634$ & East Asian & \\
\hline SRR 413668 & East Asian & \\
\hline SRR413669 & East Asian & \\
\hline SRR 413670 & East Asian & \\
\hline SRR413671 & East Asian & \\
\hline SRR 413672 & East Asian & \\
\hline SRR 413724 & East Asian & \\
\hline SRR413725 & East Asian & \\
\hline SRR4 13726 & East Asian & \\
\hline SRR 413727 & East Asian & \\
\hline SRR 413730 & East Asian & \\
\hline SRR413731 & East Asian & \\
\hline SRR 5597270 & Western European & Infant \\
\hline SRR5 597271 & Western European & Infant \\
\hline SRR5597272 & Western European & Infant \\
\hline SRR5597273 & Western European & Infant \\
\hline SRR5 597274 & Western European & Infant \\
\hline SRR 5597275 & Western European & Infant \\
\hline SRR 5597276 & Western European & Infant \\
\hline SRR 5597277 & Western European & Infant \\
\hline SRR5 597278 & Western European & Infant \\
\hline SRR5597279 & Western European & Infant \\
\hline SRR5 597280 & Western European & Infant \\
\hline SRR5597281 & Western European & Infant \\
\hline SRR5 597282 & Western European & Infant \\
\hline SRR5 597283 & Western European & Infant \\
\hline SRR 5597284 & Western European & Infant \\
\hline SRR 5597285 & Western European & Infant \\
\hline SRR 5597286 & Western European & Infant \\
\hline SRR 5597287 & Western European & Infant \\
\hline SRR5 597288 & Western European & Infant \\
\hline SRR5 597289 & Western European & Infant \\
\hline SRR 5597290 & Western European & Infant \\
\hline
\end{tabular}


bioRxiv preprint doi: https://doi.org/10.1101/2021.04.15.440092; this version posted April 16, 2021. The copyright holder for this preprint (which was not certified by peer review) is the author/funder, who has granted bioRxiv a license to display the preprint in perpetuity. It is made available under aCC-BY-NC 4.0 International license.

\begin{tabular}{|c|c|c|}
\hline SRR 5621383 & Western European & Infant \\
\hline 25621384 & Western European & Infant \\
\hline 85 & Western European & Infant \\
\hline SRR5 621386 & Western European & Infant \\
\hline RR5621 & Western European & Infant \\
\hline 8 & Western European & Infant \\
\hline 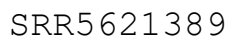 & Western European & Infant \\
\hline RR5621390 & Western European & Infant \\
\hline 6213 & Western European & Infant \\
\hline & Western European & Infant \\
\hline $\operatorname{RR} 5621393$ & Western European & Infant \\
\hline $\mathrm{R}$ & Western European & Infant \\
\hline & Western European & Infant \\
\hline $\operatorname{RR} 56213$ & Western European & Infant \\
\hline 621397 & Western European & Infant \\
\hline & Western European & Infant \\
\hline $\operatorname{RR} 56213$ & Western European & Infant \\
\hline $\operatorname{RR} 56214$ & Western European & Infant \\
\hline-1 & Western European & Infant \\
\hline $\operatorname{RR} 56214$ & Western European & Infant \\
\hline 6214 & Western European & Infant \\
\hline 22140 & Western European & Infant \\
\hline & Western European & Infant \\
\hline 406 & Western European & Infant \\
\hline RR5 621407 & Western European & Infant \\
\hline & Western European & Infant \\
\hline 6214 & Western European & Infant \\
\hline RR5621410 & Western European & Infant \\
\hline 14 & Western European & Infant \\
\hline 6 & Western European & Infant \\
\hline SRR 5621413 & Western European & Infant \\
\hline $02+4$ & Western European & Infant \\
\hline 62 & Western European & Infant \\
\hline 56 & Western European & Infant \\
\hline SRR 5621417 & Western European & Infant \\
\hline SRR 5621418 & Western European & Infant \\
\hline 56214 & Western European & Infant \\
\hline 5650021 & North American & \\
\hline 650022 & North American & \\
\hline SRR 56500 & North American & \\
\hline 5650024 & North American & \\
\hline SRR 5650025 & North American & \\
\hline SRR 5650026 & North American & \\
\hline 650027 & North American & \\
\hline SRR 5650028 & North American & \\
\hline SRR 5650029 & North American & \\
\hline SRR 5650030 & North American & \\
\hline SRR 5650031 & North American & \\
\hline SRR 5650032 & North American & \\
\hline SRR 5650033 & North American & \\
\hline SRR 5650034 & North American & \\
\hline $\mathrm{SRR}$ & North American & \\
\hline SRR 5650036 & North American & \\
\hline$R$ & North American & \\
\hline
\end{tabular}


bioRxiv preprint doi: https://doi.org/10.1101/2021.04.15.440092; this version posted April 16, 2021. The copyright holder for this preprint (which was not certified by peer review) is the author/funder, who has granted bioRxiv a license to display the preprint in perpetuity. It is made available under aCC-BY-NC 4.0 International license.

\begin{tabular}{|c|c|c|}
\hline 38 & th & $A n$ \\
\hline SRR5 650039 & North & American \\
\hline SRR 5650040 & North & American \\
\hline 650041 & North & American \\
\hline R5 650042 & North & American \\
\hline 650043 & North & American \\
\hline 5650044 & North & American \\
\hline 5650045 & North & American \\
\hline 5650046 & North & American \\
\hline 5650017 & North & American \\
\hline 5650048 & North & American \\
\hline 5650010 & North & American \\
\hline 50050 & North & American \\
\hline 57 & North & American \\
\hline 25650052 & North & American \\
\hline 650053 & North & American \\
\hline 650054 & North & American \\
\hline 650055 & North & American \\
\hline 650056 & North & American \\
\hline 650057 & North & American \\
\hline 650058 & North & American \\
\hline 650059 & North & American \\
\hline 650060 & North & American \\
\hline 5650061 & North & American \\
\hline 5650062 & North & American \\
\hline 5650063 & North & American \\
\hline 5650064 & North & American \\
\hline 5650065 & North & Ameri \\
\hline 5650066 & North & Amer \\
\hline 5650067 & North & Amer: \\
\hline 5650068 & North & Ameri \\
\hline 5650069 & North & Ameri \\
\hline 5650070 & North & Amer \\
\hline 5650071 & North & Amer \\
\hline 5650072 & North & Amer \\
\hline 5650073 & North & Amer \\
\hline 5650074 & Nor & Amer \\
\hline 5650075 & North & American \\
\hline 5650076 & North & American \\
\hline 5650077 & North & American \\
\hline 5650078 & North & Ameri \\
\hline 5650079 & North & Amer: \\
\hline 5650080 & North & Amer \\
\hline 5650081 & North & Amer \\
\hline 5650082 & North & Amer \\
\hline 650083 & North & Amer \\
\hline 650084 & North & Amer: \\
\hline 650085 & North & Amer \\
\hline 650086 & North & Amer \\
\hline 650087 & North & Ameri \\
\hline 650088 & North & Ameri \\
\hline 650089 & North & Amer \\
\hline 650090 & North & Amer: \\
\hline & & \\
\hline
\end{tabular}


bioRxiv preprint doi: https://doi.org/10.1101/2021.04.15.440092; this version posted April 16, 2021. The copyright holder for this preprint (which was not certified by peer review) is the author/funder, who has granted bioRxiv a license to display the preprint in perpetuity. It is made available under aCC-BY-NC 4.0 International license.

$\begin{array}{ll}\text { SRR5650092 } & \text { North American } \\ \text { SRR5650093 } & \text { North American } \\ \text { SRR5650094 } & \text { North American } \\ \text { SRR5650095 } & \text { North American } \\ \text { SRR5650096 } & \text { North American } \\ \text { SRR5650097 } & \text { North American } \\ \text { SRR5650098 } & \text { North American } \\ \text { SRR5650099 } & \text { North American } \\ \text { SRR5650100 } & \text { North American } \\ \text { SRR5650101 } & \text { North American } \\ \text { SRR5650102 } & \text { North American } \\ \text { SRR5650103 } & \text { North American } \\ \text { SRR5650104 } & \text { North American } \\ \text { SRR5650105 } & \text { North American } \\ \text { SRR5650106 } & \text { North American } \\ \text { SRR5650107 } & \text { North American } \\ \text { SRR5650108 } & \text { North American } \\ \text { SRR5650109 } & \text { North American } \\ \text { SRR5650110 } & \text { North American } \\ \text { SRR5650111 } & \text { North American } \\ \text { SRR5650112 } & \text { North American } \\ \text { SRR5650113 } & \text { North American } \\ \text { SRR5650114 } & \text { North American } \\ \text { SRR5650115 } & \text { North American } \\ \text { SRR5650116 } & \text { North American } \\ \text { SRR5650117 } & \text { North American } \\ \text { SRR5650118 } & \text { North American } \\ \text { SRR5650119 } & \text { North American } \\ \text { SRR5650120 } & \text { North American }\end{array}$


Source Length Phylum

adult_c_000000000801 adult c-000002956380 adult_c_000007050851 infant_c_co00000224801 infant c 000000205424 Proteobacteria

$\overline{\mathrm{P}} \mathrm{l}$ asmid
Incompatibility Group

De Novo Assembled16692 N/A

De Novo Assembled29102 N/A

De Novo Assembled26186 N/A

De Novo Assembled70278 N/A

De Novo Assembled116879

De Novo Assembled116830

infant_c_000000303989 De Novo
Plasmid $\quad$ Proteobacteria

adult_c_000008175705

adult_c_000008893813

adult_c_000003513025 Bácteroidetes

adult_c_000010613334

adult_c_000010993363

adult_c_000014022235

infant_c_000000304131

Proteobacteria

adult_c_000005638975

adult_c_000013226111

adult_c_000008893570

infant_c_co00000374357

adult_c_o00014234633

infant $\bar{c} 000000193721$

infant_c_000000466735

Proteobacteria

adult_c_000008418797

adult_c_000013226543

adult_c_000009903337

adult_c_000007050862

adult_c_000009903073

adult_c_000007869312

adult_c_000005639083

adult C 000008418239

infant_c_po0000045630

adult_c_ō00010612882

Actinobacteria

adult_c_000009903762

infant_c_o00000443464

adult_c_ $\overline{0} 00002454411$

adult_c_000010840580

adult_c_000000000515

infant_c_o00000401596

Proteobacteria

De Novo Assembled19378 N/A

De Novo Assembled77033 N/A

De Novo Assembled 153500

De Novo Assembled29291 N/A

De Novo Assembled115627

De Novo Assembled38860 N/A

De Novo Assembled21352 IncN

De Novo Assembled57539 N/A

De Novo Assembled 31783 N/A

De Novo Assembled141588

De Novo Assembled8493 N/A

De Novo Assembled15557 N/A

De Novo Assembled23740 N/A

De Novo Assembled 42634 N/A

De Novo Assembled17429 N/A

De Novo Assembled10363 N/A

De Novo Assembled 45172 N/A

De Novo Assembled25572 N/A

De Novo Assembled 62070 N/A

De Novo Assembled31256 N/A

De Novo Assembled38778 N/A

De Novo Assembled40430 N/A

De Novo Assembled33857 N/A

De Novo Assembled115232

De Novo Assembled30595 N/A

De Novo Assembled33857 N/A

De Novo Assembled51390 N/A

De Novo Assembled107190

De Novo Assembled24371 N/A

De Novo Assembled56260 N/A

De Novo Assembled 94616 IncFIB, IncFII, IncFII

infant_c_000000258786 De Novo A
Plasmid Proteobacteria

infant_c_000000381671
Proteobacteria

adult_c_000013454083

infant_c_c_000000443504

infant_c_000000460760

Proteobacteria

infant_c_000000100045

De Novo Assembled34649 N/A

De Novo Assembled75362 N/A

De Novo Assembled17351 N/A

De Novo Assembled30327 IncN

De Novo Assembled12877 N/A

Predicted Plasmid/ICE

ICE Bacteroidetes

ICE NA

ICE Bacteroidetes

ICE Firmicutes

InCFII, InCFIIA, InCFIB

IncFIB, IncFII, InCFIIA

ICE Firmicutes

ICE Firmicutes

$\mathrm{N} / \mathrm{A} \quad \mathrm{ICE}$

ICE Firmicutes

N/A ICE Firmicutes

ICE Firmicutes

Plasmid

ICE Bacteroidetes
N/A Firmicutes
N/A ICE Firmicutes
Plasmid $\quad$ Firmicutes
ICE Bacteroidetes
Plasmid NA
Plasmid

N/A Firmicutes

N/A Firmicutes

ICE Firmicutes

Plasmid Firmicutes

ICE Firmicutes

ICE Bacteroidetes

ICE Bacteroidetes

ICE Firmicutes

ICE Bacteroidetes

$\mathrm{N} / \mathrm{A} \quad \mathrm{ICE}$

ICE Bacteroidetes

ICE Bacteroidetes

N/A Firmicutes

N/A ICE Firmicutes

ICE Firmicutes

Plasmid

Plasmid

ICE Firmicutes

ICE Bacteroidetes

Plasmid

Plasmid Firmicutes 
adult_c_000012459043 adult_c_000013662038 infant_c_o00000303997 Proteobacteria adult_c_000001698700 adult_c_000009238857 adult_c_000008893666 Bacteroidetes adult_c 000000520680 infant_c_o00000174759 Proteobacteria

adult_c_000005503440 adult_c_000007050643 infant $\bar{c} 000000179675$ infant_c_000000263218 infant_c_000000401200 adult_c_o00013226115 infant_c_000000006892 adult_c $\bar{c}-\overline{0} 00012771082$ adult_c_000008693096 adult_c_000008893694 adult_c_000003513150 adult_c_000005639257 adult_c_000009906197 infant $\bar{c} 000000135411$ Proteobacteria

infant_C_000000350564 Firmicutes

adult_c_000010613001 adult_c_000001082546 adult_c_000005117488 adult_c_000009238704 adult_c_000012459167 adult_c_000009903689 adult_c_000001082227 adult_c_000011135113 adult_c_000007288282 infant_c_000000374099 Proteobacteria adult_c_000001699985 adult_c_000006007668 infant_c_o00000304100 Proteobacteria

adult_c_000002454352 Bácteroidetes

adult_c_000010613216 adult_c_000013455219 infant $\bar{c} 000000443434$ adult_c $\bar{c} 00002012993$ adult_c_000005117674 infant_c_co00000413404 Proteobacteria adult_c_000010252652 adult_c_000002454722
De Novo Assembled34522 N/A

De Novo Assembled36865 N/A

De Novo Assembled107799

De Novo Assembled24064 N/A

De Novo Assembled35388 N/A

De Novo Assembled103064

De Novo Assembled26481 N/A De Novo Assembled33756 IncN

De Novo Assembled61243 N/A

De Novo Assembled 64132 N/A

De Novo Assembled 46283 N/A

De Novo Assembled 9418 N/A

De Novo Assembled112299

De Novo Assembled31569 N/A

De Novo Assembled53838 N/A

De Novo Assembled 44787 N/A

De Novo Assembled51519 N/A

De Novo Assembled 96827 N/A

De Novo Assembled83231 N/A

De Novo Assembled24818 N/A

De Novo Assembled8543 N/A

De Novo Assembled $37340 \mathrm{~N} / \mathrm{A}$

De Novo Assembled57158 rep_cluster_1027 Plasmid

De Novo Assembled $66201 \mathrm{~N} / \mathrm{A}$

De Novo Assembled34288 N/A

De Novo Assembled49572 N/A

De Novo Assembled59031 N/A

De Novo Assembled22640 N/A

De Novo Assembled32405 N/A

De Novo Assembled80719 N/A

De Novo Assembled32386 N/A

De Novo Assembled $37582 \mathrm{~N} / \mathrm{A}$

De Novo Assembled 40147 IncFII

ICE Bacteroidetes

ICE Firmicutes

ICE Firmicutes

ICE Firmicutes

Plasmid Firmicutes

ICE Firmicutes

ICE Actinobacteria

ICE Bacteroidetes

ICE Bacteroidetes

Plasmid

De Novo Assembled11213 N/A ICE Firmicutes

De Novo Assembled37187 N/A ICE Firmicutes

De Novo Assembled29492 IncN Plasmid

De Novo Assembled112748

$\mathrm{N} / \mathrm{A} \quad \mathrm{ICE}$

De Novo Assembled38451 N/A

De Novo Assembled13773 N/A

De Novo Assembled107865

ICE Actinobacteria

Plasmid Firmicutes

$\mathrm{N} / \mathrm{A} \quad \mathrm{ICE} \quad \mathrm{NA}$

De Novo Assembled28007 N/A ICE Bacteroidetes

De Novo Assembled 32289 N/A ICE Bacteroidetes

De Novo Assembled67821 IncFII Plasmid

De Novo Assembled 32445 N/A

ICE Bacteroidetes

De Novo Assembled16059 N/A
Plasmid

Firmicutes 
adult_c_000008895263 adult_c_000002012951 adult_c_-000002808617 infant_c_o00000174731 adult_c $\bar{c}$ - 00003513241 adult_c_000010840695 adult c 000013094215 infant_c_co00000336129 Proteobacteria adult_c_000001698711 adult_c_000003212341 infant_c_co00000128696 Proteobacteria adult_c 000009903100 adult_c_000005118029 adult_c_000008893491 Bacteroidetes infant_c_000000286772 Proteobacteria infant_c_000000286787 Proteobacteria adult_c 000007869171 adult_c_000014022244 adult_c_000009238639 adult_c 000009903082 adult_c_000009903659 infant_c_o00000128700 adult $\bar{c} \overline{0} 00004735184$ adult_c_000012458878 Bácteroidetes adult_c_000008893965 infant_c_000000023493 adult_c $\bar{c} 00008893684$ infant_c_000000125885 Proteobacteria

infant_c_000000152023 Proteobacteria adult_c_000008895641 adult_c_000002955808 adult_c_000002454444 adult_c_000006501409 adult_c_000013661551 adult_c_000010840712 adult_c_000009904612 infant_c_co00000245980 adult_c_-o00012771341 adult_c_000009903079 adult_c_000013454101 infant_c_co00000135483 infant_c_000000460740 Proteobacteria infant_c_000000128584 Proteobacteria $\begin{array}{llll}\text { De Novo Assembled23741 N/A } & \text { ICE } & \text { Firmicutes } \\ \text { De Novo Assembled56733 N/A } & \text { ICE } & \text { Bacteroidetes } \\ \text { De Novo Assembled19542 N/A } & \text { N/A } & \text { Firmicutes } \\ \text { De Novo Assembled51224 N/A } & \text { ICE } & \text { Bacteroidetes } \\ \text { De Novo Assembled62013 N/A } & \text { ICE } & \text { Bacteroidetes } \\ \text { De Novo Assembled46579 N/A } & \text { ICE } & \text { Bacteroidetes } \\ \text { De Novo Assembled67660 N/A } & \text { ICE } & \text { Firmicutes }\end{array}$ De Novo Assembled71136 IncFIIA, IncFII Plasmid

De Novo Assembled23673 N/A ICE Firmicutes De Novo Assembled11179 N/A ICE Bacteroidetes De Novo Assembled19795 N/A Plasmid

De Novo Assembled60337 N/A ICE Bacteroidetes De Novo Assembled16546 N/A ICE Firmicutes De Novo Assembled229430 N/A ICE

De Novo Assembled48259 IncFII Plasmid

De Novo Assembled42384 IncFII, IncFIIA Plasmid

De Novo Assembled59560 N/A ICE Firmicutes

De Novo Assembled33311 N/A ICE Firmicutes

De Novo Assembled80023 N/A ICE Firmicutes

De Novo Assembled $61387 \mathrm{~N} / \mathrm{A}$ ICE Firmicutes

De Novo Assembled33280 N/A Plasmid Firmicutes

De Novo Assembled19484 N/A Plasmid NA

De Novo Assembled25317 N/A ICE Bacteroidetes

De Novo Assembled173394 N/A ICE

De Novo Assembled64224 N/A N/A Firmicutes

De Novo Assembled183154 N/A ICE NA

De Novo Assembled $98121 \mathrm{~N} / \mathrm{A}$ ICE Bacteroidetes

De Novo Assembled74291 IncFII Plasmid

De Novo Assembled61599 N/A Plasmid

De Novo Assembled19809 N/A

De Novo Assembled $47546 \mathrm{~N} / \mathrm{A}$

Plasmid Firmicutes

De Novo Assembled38559 N/A

ICE Firmicutes

N/A Firmicutes

De Novo Assembled17714 N/A

ICE Bacteroidetes

De Novo Assembled58641 N/A

De Novo Assembled43228 N/A

De Novo Assembled16464 N/A

De Novo Assembled11895 N/A

De Novo Assembled20975 N/A

De Novo Assembled 61764 N/A

De Novo Assembled 67740 N/A

De Novo Assembled21481 N/A

ICE

$I C E$

Firmicutes

Bacteroidetes

N/A Firmicutes

Plasmid Firmicutes

ICE Firmicutes

ICE Firmicutes

ICE Firmicutes

Plasmid Firmicutes

Plasmid

De Novo Assembled141568

$\mathrm{N} / \mathrm{A} \quad \mathrm{N} / \mathrm{A}$ 
infant_c_000000262443 Proteobacteria adult_c_000013665368 infant_c_co00000099905 Proteobacteria

adult_c_000013661289 adult_c_000002808141 infant_c_o00000374119 Proteobacteria adult_c_000000358633 infant_c_co00000358770 Proteobacteria adult_c_000008895425 adult c-000001698149 infant_c_co00000414145 Proteobacteria

adult_c_000002205632 adult_c_000013094287 adult_c_000004493597 adult_c_000001082312 infant_c_co00000174710 adult $\bar{c} \overline{0} 00013661550$ infant_c_c000000401206 adult_c_o00008896141 adult c 000007629373 infant_c_o00000466798 adult_c_ō00008175023 adult_c 000008418252 adult_c_000013662355 infant_c_co00000039988 adult_c_- $\overline{0} 00013662066$ adult_c_000008894078 adult_c_000009902917 adult_c_000013661363 adult c-000008175866 infant_c_o00000466760 adult_c_o00001697990 Bácteroidetes

adult_c_000010993709 adult_c_-000012458882 Bācteroidetes adult_c_000008894963 adult_c_000010435350 adult_c_000008895517 adult_c_000007050965 adult_c_000002955364 adult_c_000009238927 infant_c_000000262451 infant_c_000000020966 $\overline{\mathrm{NA}}$

adult_c 000013662340 adult_c_000002808176 infant_c_co00000393866 adult_c﹎o- 000012459381
De Novo Assembled43918 IncFIIA, IncFII Plasmid

De Novo Assembled9534 N/A N/A Firmicutes De Novo Assembled57596 N/A Plasmid

De Novo Assembled96892 N/A ICE Firmicutes De Novo Assembled89845 N/A ICE Firmicutes De Novo Assembled33840 IncFII, IncFII Plasmid

De Novo Assembled22343 N/A De Novo Assembled11488 N/A

N/A Firmicutes Plasmid

De Novo Assembled22015 N/A De Novo Assembled58646 N/A De Novo Assembled119890

$\begin{array}{ll}\text { N/A } & \text { Firmicutes } \\ \text { ICE } & \text { Bacteroidetes } \\ \text { N/A } & \text { ICE }\end{array}$

De Novo Assembled30359 N/A De Novo Assembled $47286 \mathrm{~N} / \mathrm{A}$

ICE Firmicutes ICE Firmicutes ICE Firmicutes ICE Firmicutes ICE Bacteroidetes ICE Bacteroidetes ICE Firmicutes ICE Firmicutes ICE Firmicutes Plasmid Firmicutes ICE Bacteroidetes ICE Bacteroidetes ICE Bacteroidetes Plasmid Firmicutes ICE Firmicutes ICE Bacteroidetes ICE Firmicutes ICE Firmicutes ICE Bacteroidetes Plasmid Firmicutes $\mathrm{N} / \mathrm{A} \quad \mathrm{ICE}$

De Novo Assembled17418 N/A De Novo Assembled143297

\section{N/A Actinobacteria}

$\mathrm{N} / \mathrm{A} \quad \mathrm{ICE}$

De Novo Assembled27851 N/A De Novo Assembled14598 N/A

De Novo Assembled20996 N/A

De Novo Assembled20627 N/A

De Novo Assembled104777

De Novo Assembled30401 N/A

De Novo Assembled $42131 \mathrm{~N} / \mathrm{A}$

ICE Firmicutes

N/A Firmicutes

ICE Firmicutes

$\mathrm{N} / \mathrm{A} \quad \mathrm{NA}$

N/A ICE Firmicutes

N/A Firmicutes

ICE Firmicutes

De Novo Assembled26734 IncFIIA, IncFII Plasmid

De Novo Assembled29139 N/A De Novo Assembled $60245 \mathrm{~N} / \mathrm{A}$ De Novo Assembled16646 N/A De Novo Assembled14601 N/A
ICE Firmicutes
ICE Actinobacteria
Plasmid NA
N/A Firmicutes 
adult_c_000009902758

adult_c_-000013661145

Bäcteroidetes

infant_c_000000045669

adult_c_ 000006501252

infant_c_o00000045576 Plasmid

Prot

De Novo Assembled120984

De Novo Assembled162159

De Novo Assembled17351 N/A

De Novo Assembled29980 N/A

obacteria $\begin{array}{lll}\text { N/A } & \text { ICE } & \text { Firmicutes } \\ \text { N/A } & \text { ICE }\end{array}$

ICE Bacteroidetes

ICE Bacteroidetes

adult_c_000001084269

adult_c_000009903018

adult_c_000005117362

infant_c_o00000263281

adult_c $\bar{c} \overline{0} 00008418315$

infant_c_o00000374084

Proteobacteria

adult_c_000008894303

adult_c_000008893599

De Novo Assembled6845 N/A

De Novo Assembled $66737 \mathrm{~N} / \mathrm{A}$

De Novo Assembled73110 N/A

De Novo Assembled8838 N/A

De Novo Assembled34171 N/A

De Novo Assembled $45874 \mathrm{~N} / \mathrm{A}$

De Novo Assembled $46714 \mathrm{~N} / \mathrm{A}$

De Novo Assembled 124528

infant_c_o00000245609 De Novo Assembled116687 $\overline{\mathrm{P}} \mathrm{i}$ asmid

Proteobacteria

adult_C_000001082544 De Novo Assembled34436 N/A ICE

infant_c_o00000099900

Proteobacteria

adult_c_000009796704

adult_c_000008894505

infant_c_000000381698

Proteobacteria

adult_c_000009904452

adult_c_000001082609

infant_c__o00000125876

Proteobacteria

adult_c_000007629525

adult_c_000009796510

adult_c_000013662810

infant_c_c_000000045650

adult_c $\bar{c} \overline{0} 00013661442$

infant_c_co00000262436

adult_c_ $\bar{c} 00000000253$

De Novo Assembled71603 IncFII

Plasmid Firmicutes

ICE Firmicutes

ICE Bacteroidetes

N/A Proteobacteria

ICE Firmicutes

Plasmid

ICE Firmicutes

N/A ICE Firmicutes

InCFIB, InCFII, InCFIIA

De Novo Assembled13577 N/A

De Novo Assembled39123 N/A

De Novo Assembled27397 N/A

ICE Firmicutes

ICE Firmicutes

Plasmid

De Novo Assembled18368 N/A Plasmid Firmicutes

De Novo Assembled30877 N/A ICE Actinobacteria

De Novo Assembled83321 IncFII, IncFII Plasmid

De Novo Assembled31651 N/A ICE Firmicutes

De Novo Assembled44783 N/A ICE Firmicutes

De Novo Assembled22028 N/A N/A Firmicutes

De Novo Assembled23965 N/A Plasmid Firmicutes

De Novo Assembled69550 N/A ICE Bacteroidetes

De Novo Assembled47883 N/A ICE Firmicutes

De Novo Assembled37154 N/A ICE Bacteroidetes

infant_c_000000413394 De Novo Assembled 92749 IncFIB, IncFIIA, IncFII

$\overline{\mathrm{P}} \mathrm{l}$ asmid $\quad$ Proteobacteria

infant_C_000000074962 De Novo Assembled18189 N/A

adult_c_ $\bar{C} 00008896282$

adult_c_000014234155

adult_c_000009239288

infant_c_co00000125966

adult_c_ $\overline{0} 00009902672$

adult_c_000009905953

infant_c_co00000135388

adult_c $\bar{C}$ - 00008895240

adult_c_000006501244

adult_c_000013661838

adult_c_000002955701

adult_c_000001082674

adult_c_000002454751

adult_c_000008895289

De Novo Assembled14813 N/A

De Novo Assembled156889

De Novo Assembled19013 N/A

De Novo Assembled33556 N/A

De Novo Assembled175108

De Novo Assembled 9200 N/A

De Novo Assembled 49775 N/A

De Novo Assembled24092 N/A

De Novo Assembled 31120 N/A

De Novo Assembled 43940 N/A

Plasmid

Plasmid

Firmicutes

N/A ICE Firmicutes

Plasmid Firmicutes

Plasmid Firmicutes

N/A ICE Firmicutes

N/A Firmicutes

Plasmid Firmicutes

ICE Bacteroidetes

ICE Firmicutes

ICE Bacteroidetes

De Novo Assembled54082 N/A

ICE

Firmicutes

De Novo Assembled27406 N/A

ICE

Firmicutes

De Novo Assembled15279 N/A

ICE

NA

De Novo Assembled23452 N/A

$I C E$

Firmicutes

De Novo Assembled49692 N/A

ICE

Firmicutes 
adult_c_000003513629 adult_c_000004735478 adult_c_000010993568 adult c-000013455746 adult_c_000001082172 adult_c_000003211468 adult c 000001083087 adult_c_000007050912 infant $\bar{c} 000000128685$ adult_c_ō00001699674 adult_c_000010613012 adult_c_000008895151 adult_c_000005117827 adult_c_000002808113 infant_c_co00000122023 Proteobacteria adult c 000009797086 infant_c_co00000135416 Proteobacteria adult_c_000000359312 adult_c_000003798815 adult_c-000012458990 adult_c_000008893944 adult_c_000012458901 adult_c 000008175666 adult_c_000001083021 adult_c_000013662409 infant_c_000000023497 adult_c_ $\overline{0} 00008176248$ adult_c_000009239399 adult_c_000002957117 adult_c_000014234156 Bacteroidetes

adult_C_000014234181 adult_c_000009238951 adult_c_000008893711 adult_c_000002955301 Bacteroidetes adult_c_000006007941 infant_c_co00000262355 adult_c_ 000002013135 adult_c_000002808107 adult_c_000002012989 adult_c_000014234297 adult_c_000003513217 infant_c_co00000099932 Proteobacteria adult c 000010434937 adult_c_000010840574 adult_c_000008175049 adult_c_000014684369 adult_c_000002956018 adult_c_000002454576 adult_c_000005639089
De Novo Assembled30276 N/A

De Novo Assembled10358 N/A

De Novo Assembled26581 N/A

De Novo Assembled10484 N/A

De Novo Assembled124710

De Novo Assembled68876 N/A

De Novo Assembled16545 N/A

De Novo Assembled23167 N/A

De Novo Assembled24007 N/A

De Novo Assembled 12686 N/A

De Novo Assembled 63409 N/A

De Novo Assembled25207 N/A

De Novo Assembled23886 N/A

De Novo Assembled139972

De Novo Assembled 64306 IncFII, IncFII

De Novo Assembled6941 N/A De Novo Assembled34465 IncN

De Novo Assembled9138 N/A

De Novo Assembled24351 N/A

De Novo Assembled $44258 \mathrm{~N} / \mathrm{A}$

De Novo Assembled65551 N/A

De Novo Assembled 92984 N/A

De Novo Assembled20411 N/A

De Novo Assembled17602 N/A

De Novo Assembled27851 N/A

De Novo Assembled109183

De Novo Assembled11170 N/A

De Novo Assembled16689 N/A

De Novo Assembled18579 N/A

De Novo Assembled151741

De Novo Assembled100984

De Novo Assembled28644 N/A

De Novo Assembled 92672 N/A

De Novo Assembled144197

De Novo Assembled17932 N/A

De Novo Assembled141698

De Novo Assembled12565 N/A

De Novo Assembled167082

De Novo Assembled29321 N/A

De Novo Assembled39017 N/A

De Novo Assembled 65632 N/A

De Novo Assembled34649 N/A

De Novo Assembled81995 N/A

De Novo Assembled120864

De Novo Assembled54704 N/A

De Novo Assembled50193 N/A

De Novo Assembled37926 N/A

De Novo Assembled22599 N/A

De Novo Assembled37566 N/A
Plasmid

Plasmid

ICE Actinobacteria

Plasmid Firmicutes

ICE Firmicutes

Plasmid Firmicutes

N/A ICE Firmicutes

ICE Bacteroidetes

ICE Firmicutes

ICE Bacteroidetes

Plasmid Firmicutes

ICE Firmicutes

ICE Actinobacteria

ICE Firmicutes

ICE Bacteroidetes

N/A ICE Firmicutes Plasmid

Firmicutes

Plasmid Firmicutes

ICE Bacteroidetes

ICE Firmicutes

ICE Firmicutes

ICE Firmicutes

ICE Bacteroidetes

ICE Firmicutes

ICE Firmicutes

N/A ICE NA

ICE Bacteroidetes

ICE Bacteroidetes

ICE Firmicutes

$\mathrm{N} / \mathrm{A} \quad \mathrm{ICE}$

N/A ICE Firmicutes

ICE Bacteroidetes

ICE Bacteroidetes

$\mathrm{N} / \mathrm{A} \quad \mathrm{ICE}$

ICE Firmicutes

N/A ICE Firmicutes

ICE Bacteroidetes

N/A ICE Firmicutes

ICE Bacteroidetes

ICE Bacteroidetes

ICE Bacteroidetes

Plasmid

ICE Firmicutes

N/A ICE Firmicutes

ICE Actinobacteria

ICE Firmicutes

ICE Bacteroidetes

ICE Bacteroidetes

ICE Bacteroidetes 
infant_c_000000381620 adult_c_ $\overline{0} 00001082753$ adult_c_000010612878 Bacteroidetes adult_c_000013454543 adult_c_000004735154 adult c 000001699266 adult_c_000013661185 adult_c_000013094138 adult_c_000004174691 adult_c_000013661873 adult_c_000009238592 Bácteroidetes

adult_c 000009796433 adult_c_000000000329 adult_c_000013661385 infant_c_o00000094061 infant_c_000000304115 Proteobacteria adult_c_000009239534 infant_c_co00000014380 Proteobacteria

infant_c_000000414188 Proteobacteria adult_c 000013454086 adult_c_000002955876 adult_c_000007050585 Bácteroidetes adult_C_000002454588 adult_c_000009238662 infant_c_co00000045570 adult_c_ō00003211379 Bacteroidetes

adult_c 000009903506 adult c 000008893658 adult_c 000003211643 infant_c_000000350544 adult $\bar{c} \overline{0} 00001698238$ adult_c_000004174622 adult_c_000002955253 infant_c_000000179771 Proteobacteria adult_c_000010613291 adult_c_000001082153 infant_c_o00000023502 $\overline{\mathrm{P}} \mathrm{l}$ àsmid Prot adult_c_000002454345 Bacteroidetes

adult_c_000003211382 Bácteroidetes adult_c 000001704041 adult_c_000008894504 infant_c_o00000135456 adult_c﹎ō00002205865
De Novo Assembled74815 N/A De Novo Assembled24836 N/A De Novo Assembled115717

De Novo Assembled24016 N/A De Novo Assembled28396 N/A De Novo Assembled15581 N/A De Novo Assembled130772

De Novo Assembled297288

De Novo Assembled15470 N/A

De Novo Assembled 42969 N/A

De Novo Assembled131124

De Novo Assembled169754

De Novo Assembled31978 N/A

De Novo Assembled77068 N/A

De Novo Assembled20239 N/A

De Novo Assembled26240 N/A

De Novo Assembled14669 N/A

De Novo Assembled161217

De Novo Assembled54211 IncFII, IncFIIA Plasmid

De Novo Assembled74611 N/A De Novo Assembled 44035 N/A De Novo Assembled165180

ICE Firmicutes

ICE Firmicutes

$\mathrm{N} / \mathrm{A} \quad \mathrm{ICE}$

De Novo Assembled22063 N/A

De Novo Assembled71274 N/A

De Novo Assembled107995

De Novo Assembled185861

De Novo Assembled38356 N/A

De Novo Assembled105559

De Novo Assembled32289 N/A

De Novo Assembled101395

De Novo Assembled46983 N/A

De Novo Assembled32193 N/A

De Novo Assembled 942574

De Novo Assembled29144 IncN

Plasmid

ICE Firmicutes

$\mathrm{N} / \mathrm{A}$ ICE NA

$\mathrm{N} / \mathrm{A} \quad \mathrm{ICE}$

ICE Firmicutes

N/A ICE Firmicutes

ICE Bacteroidetes

N/A ICE Firmicutes

ICE Firmicutes

N/A Firmicutes

N/A ICE Firmicutes

Plasmid

De Novo Assembled32261 N/A ICE Bacteroidetes

De Novo Assembled171495 N/A ICE Firmicutes

De Novo Assembled 66584 rep_cluster_398,IncP obacteria

De Novo Assembled173864

De Novo Assembled168765

$\mathrm{N} / \mathrm{A} \quad \mathrm{ICE}$

$\mathrm{N} / \mathrm{A} \quad \mathrm{ICE}$

De Novo Assembled4786 N/A

De Novo Assembled39125 N/A

De Novo Assembled25807 N/A

De Novo Assembled16855 N/A
Plasmid

ICE Firmicutes

ICE Firmicutes

ICE Firmicutes 
adult_c_000009239549 adult_c_000001082145 infant_c_o00000245907

infant_c_000000381632 Proteobacteria adult_c_000013094433 adult_c_000013225982 adult_c_000014234441 adult_c_000008893979 adult_c_000006501488 adult_c_000005503414 adult_c_000007629582 adult_c_000002955819 infant_c_o00000205471 adult_c $\bar{c}$ 000008693386 adult_c_000009903260 adult_c_000003798789 adult_c_000000000074 infant_c_c000000286907 adult_c_ $\overline{0} 00009239761$ adult_c_- 000004174578 adult_c_000008894223 adult_c_000009903210 adult_c_000010612941 adult_c 000013661766 adult_c_000007629995 infant_c__000000179786 Proteobacteria adult_c_000003513463 adult_c_000014234210 adult_c_000009903212 adult_C_000007869127 adult_c_000013661423 infant_c_co00000443473 infant_c_000000414439 infant_c_000000174700 $\overline{\mathrm{P}} \mathrm{l} \overline{\mathrm{s} m i d}$

Prot adult_c_000008174984 adult_c_000008174892 adult_c_000004174614 adult_C_000003211523 adult_c_000008174901 adult_c_000011259081 adult_c_000001698339 infant_c_o00000205574 adult_c $\bar{c}-\overline{0} 00014234709$ adult_c_000002454450 infant_c_000000006864 infant C 000000443438
De Novo Assembled14447 N/A

De Novo Assembled207820

De Novo Assembled14359 N/A

De Novo Assembled57596 N/A

De Novo Assembled29755 N/A

De Novo Assembled60337 N/A

De Novo Assembled22864 N/A

De Novo Assembled 63029 N/A

De Novo Assembled15048 N/A

De Novo Assembled131634

De Novo Assembled27525 N/A

De Novo Assembled 47210 N/A

De Novo Assembled53525 N/A

De Novo Assembled15393 N/A

De Novo Assembled 49262 N/A

De Novo Assembled26456 N/A

De Novo Assembled72362 N/A

De Novo Assembled17517 N/A

De Novo Assembled12460 N/A

De Novo Assembled80313 N/A

De Novo Assembled 49828 N/A

De Novo Assembled52753 N/A

De Novo Assembled83231 N/A

De Novo Assembled 47674 N/A

De Novo Assembled15478 N/A

De Novo Assembled27381 N/A

De Novo Assembled40178 N/A De Novo Assembled68925 N/A De Novo Assembled52550 N/A De Novo Assembled86274 N/A De Novo Assembled72635 N/A De Novo Assembled24277 N/A De Novo Assembled11927 N/A De Novo Assembled138940 eobacteria

De Novo Assembled66637 N/A De Novo Assembled 97068 N/A De Novo Assembled40601 N/A De Novo Assembled 49769 N/A De Novo Assembled $90964 \mathrm{~N} / \mathrm{A}$ De Novo Assembled30473 N/A De Novo Assembled38321 N/A De Novo Assembled23395 N/A De Novo Assembled14234 N/A De Novo Assembled 37558 N/A De Novo Assembled73148 N/A De Novo Assembled 92163 IncFII, IncFIB, IncFIIA Plasmid Proteobacteria

adult_c_000001082340 De Novo Assembled53565 N/A infant_c_o00000006854 De Novo Assembled87412 InCFII, IncFIA, rep_cluster_959 Plasmid adult_C_000002957226 De Novo Àssembled17755 N/A
ICE Firmicutes

N/A ICE Firmicutes

Plasmid NA

Plasmid

N/A Firmicutes

ICE Bacteroidetes

ICE Bacteroidetes

ICE Firmicutes

N/A Bacteroidetes

N/A ICE Firmicutes

ICE Firmicutes

ICE Firmicutes

ICE NA

ICE Firmicutes

N/A Firmicutes

ICE Bacteroidetes

ICE Firmicutes

Plasmid Firmicutes

N/A Firmicutes

ICE Bacteroidetes

ICE Firmicutes

ICE Firmicutes

ICE Bacteroidetes

ICE Firmicutes

Plasmid Firmicutes

Plasmid

ICE Bacteroidetes
N/A Firmicutes
ICE Firmicutes
ICE Bacteroidetes
N/A Firmicutes
Plasmid Firmicutes
Plasmid Firmicutes
InCFI, InCFIIA

ICE Bacteroidetes

ICE Bacteroidetes

ICE Bacteroidetes

ICE Firmicutes

ICE Actinobacteria

ICE Firmicutes

ICE Bacteroidetes

Plasmid NA

Plasmid Firmicutes

ICE Firmicutes

ICE NA

ICE Actinobacteria

Proteobacteria

ICE Firmicutes 
infant_c_000000128663 De Novo Assembled32640 N/A Plasmid

Proteobacteria

UMGS221_SRR1449329_bin.5_Tenericutes_Mollicutes_NA_NA_NA_NODE_3_length_12 $7005 \mathrm{cov} 7.53875$ Genome Set 127005 N/A I $\overline{C E}$ Tenericutes

14207_7_64_Firmicutes_Clostridia_Clostridiales_NA_NA_.14207_7_64.4

Génome set $2666 \overline{2} 5 \quad$ N/A ${ }^{-}$ICE Firmicutes

NZ_AEXR01000016.1_Eggerthella_sp._HGA1_contig00024_whole_genome_shotgun_ sequence Genome set $222459^{-}$N/A ICE Actinobacteria

12718_789 Actinobacteria_Actinobacteria_Coriobacteriales_Coriobacteriace

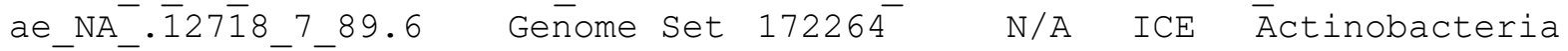
18048_2_85_Firmicutes_Clostridia_Clostridiales_Peptostreptococcaceae_Clos

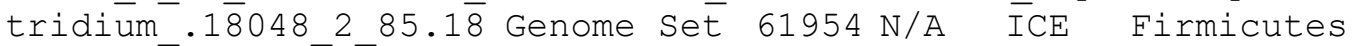

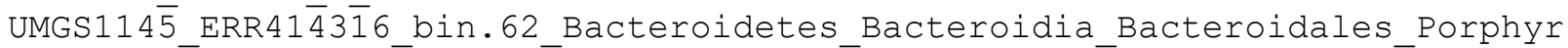
omonadacēee_NA_NODE_110_lēength_96759_coV_44.976857 Genome set $96 \overline{759}$ N/A

ICE Bacteroidetes

UMGS426_SRR5032278_bin.10_Firmicutes_Clostridia_Clostridiales_Oscillospir

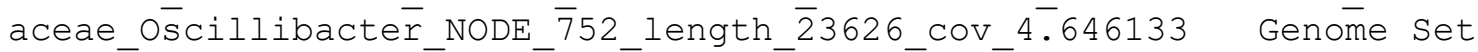

$23626 \mathrm{~N} / \mathrm{A} \quad \mathrm{ICE} \quad$ Firmicutes

UMGS147_SRR5091520_bin.30_Firmicutes_Clostridia_Clostridiales_Lachnospira ceae_Ros̄eburia_NODĒ_298_lēength_51670_Cov_25.6833̄87 Genome Set 51670 N/A

ICE Firmicutes

NZ_JH590969.1_Erysipelotrichaceae_bacterium_6_1_45_genomic_scaffold_super cont1.2_wholē_genome_shotgun_sequence Genome $\overline{\text { Set }} 120764 \overline{6}$ N/A ${ }^{-}$ICE Tenericutes

UMGS1468 ERR1293763 bin.7 Tenericutes Mollicutes Erysipelotrichales Erysi pelotrichaceae_Solobacterium_NODE_756_length_15276_cov_3.311609

Genome Set 15276 N/A N/A Tenericutes

NZ_GG657561.1_Holdemania_filiformis_DSM_12042_genomic_scaffold_Scfld10_W hole_genome_shotgun_sequence Genome $\overline{S e t}^{-} 68466 \overline{8} \quad \mathrm{~N} / \overline{\mathrm{A}}$ ICE

Tenerícutes

UMGS985_ERR414362_bin.39_Firmicutes_Clostridia_Clostridiales_Lachnospirac

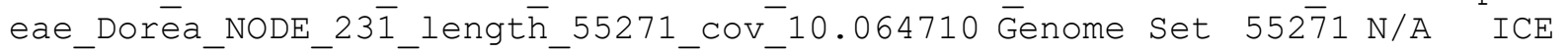

Firmicutes

UMGS1144_SRR5032340_bin.7_Firmicutes_Clostridia_Clostridiales_NA_NA_NODE 140_length 45014 cov $17.3 \overline{3} 7285$ Geñome set $4 \overline{5014}$ N/A N/A Firmicutes UMGSי 803 SRR $51274 \overline{1} 1$ bin.10_Firmicutes_Clostridia_Clostridiales_Peptostrept ococcacēae_Clostrid̄ium_NOD̄E_1440_length_9658_cov_8.665209Genome Set 9658

N/A ICE Firmicutes

14207_7_23_Bacteroidetes_Bacteroidia_Bacteroidales_Bacteroidaceae_Bactero ides_.1 $1 \overline{4} 20 \overline{7} 7$ 7_23.47 Génome set $28 \overline{5} 31 \mathrm{~N} / \mathrm{A}$ ICE Bacteroidetes NZ_A $\bar{B} I X 0200 \overline{0} 0 \overline{0} 2.1$ Bifidobacterium_dentium_ATCC_27678_B_dentium-

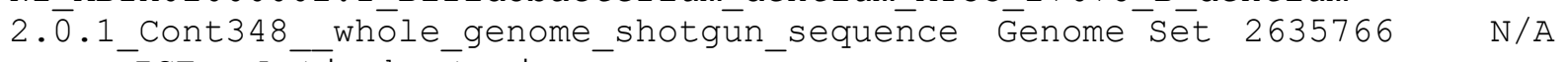
ICE Actinobacteria

NZ_DS996921.1_[Bacteroides]_pectinophilus_ATCC_43243_Scfld_02_1_genomic_s caffold_whole_genome_shotgun_sequence Genome $\bar{e}^{-}$Set $252838 \overline{1}^{-}-\overline{\mathrm{N}} / \mathrm{A}$ ICE Firmicutes

NZ_GL988014.1_Fusobacterium_sp._D12_genomic_scaffold_supercont2.3_whole_ geñome_shotguñ_sequence - Genome Set $195 \overline{4} 08 \quad$ N/A ICE

Fusobacteria

20298_3_64_Firmicutes_Clostridia_Clostridiales_Ruminococcaceae_NA_.20298_ 3_64.10- Genome Set 125256 N/A ICE Firmicutes

UM̄GS231_SRR4033073_bin.3_Firmicutes_Clostridia_Clostridiales_Ruminococcac eae_NA_NODE_1_length_437286_COV_34.278775 Genome Set $437 \overline{2} 86$ N/A $\overline{\text { ICE }} \overline{\text { Firmicutes }}$ 
UMGS618_SRR4408228_bin.21_Firmicutes_Clostridia_Clostridiales_NA_NA_NODE_ 408 lenğth_26456_cov_10.44488 Genome Set 26456 N/A ICE Firmicūtes UMG $\bar{S} 106$ SRR $60544 \overline{9} 3$ bìn.13_Firmicutes_Clostridia_Clostridiales_NA_NA_NODE_ 2_length_236515_cov $20.27 \overline{6} 787$ Genome Set $2 \overline{3} 6515 \quad$ N/A - I $\bar{C} E$ Firmicutes

20298_2_28_Firmicutes_Clostridia_Clostridiales_NA_NA_.20298_2_28.1 Genome set $1109 \overline{6} 66 \quad$ N/A ICE Firmicutes

14207_7_54_Firmicutes_Clostridia_Clostridiales_NA_NA_.14207_7_54.1 Genome set $1366 \overline{1} 17 \quad$ N/A ${ }^{-}$ICE Firmicütes

NZ_CAPH01000018.1_Alistipes_ihumii_AP11_whole_genome_shotgun_sequence Genome Set $279520-$ N/A ICE Bacteroidetes

UMGS792_SRR5580076_bin.53_Firmicutes_Clostridia_Clostridiales_NA_NA_NODE_

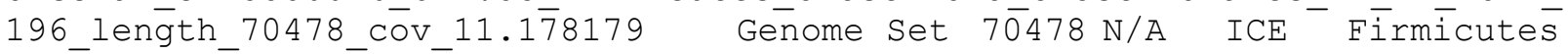
UMG $\bar{S} 1776$ SRR $5558 \overline{1} 95$ bin.42 Firmicutes Clostridia Clostridiales NA NA NODE

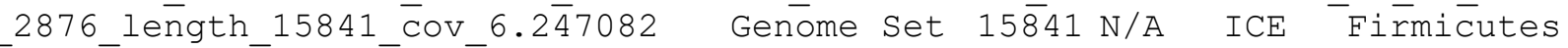

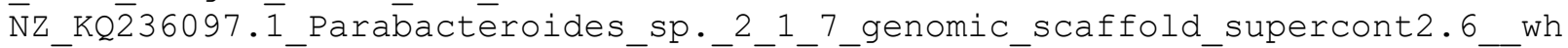
olè_genome_shōtgun_sequence Genome set 565040 N/A ICE

Bacteroidetes

NZ_GG705175.1_Bacteroides_sp._2_1_22_genomic_scaffold_supercont1.3_whole _gènome_shotgün_sequence Geñome set $1143 \overline{6} 23$ N/A ICE

Bàcteroidetes

13414_6_68_Firmicutes_Clostridia_Clostridiales_Lachnospiraceae_NA_.13414 6 68.5 - Genome Set 283444 - N/A ICE Firmicutes

20̄298_3_65_Firmicutes_Clostridia_Clostridiales_Lachnospiraceae_NA_.20298_ $365 . \overline{5}-$ Genome Set $263814-$ N/A ICE Firmicutes

NZ__HE978601.1_Brevibacterium_sp._JC43_genomic_scaffold_scaffold00002_wh olē_genome_shötgun_sequence Genome set $59115 \overline{5} \quad \mathrm{~N} / \overline{\mathrm{A}}$ ICE

Actinobactería

NZ_GL520136.1_Erysipelotrichaceae_bacterium_3_1_53_genomic_scaffold_super

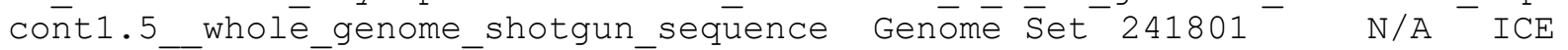
Tenericutes

20298_3_62_Firmicutes_Clostridia_Clostridiales_Peptostreptococcaceae_Clos tridium_.20̄298_362.4 Genome SeE $367400 \quad \bar{N} / A$ ICE Firmicutes UMGS846_ERR11906 $\overline{3} 9$ _bin.35_Bacteroidetes_Bacteroidia_Bacteroidales_Prevote

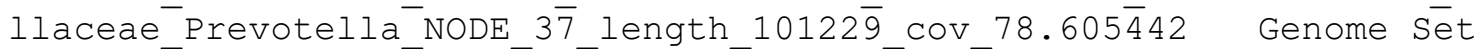
$1 \overline{0} 1229 \quad \mathrm{~N} / \mathrm{A} \quad \overline{\mathrm{I}} \mathrm{CE}$ Bacteroidetes

20287_6_63_Firmicutes_Clostridia_Clostridiales_Lachnospiraceae_NA_.20287_ 6_63. $\overline{2}^{-}$- Genome Set 13507 N/A ${ }^{-}$ICE Firmicütes

UMMGS651_SRR2912799 bin.35_Actinobacteria_Actinobacteria_Coriobacteriales Coriobacteriaceae_Collinsēlla_NODE_607_lèngth_30769_cov_26.239435

Genome set 30769 N/A N/A Āctinobacteria

UMGS1413_SRR5579982_bin.24_Firmicutes_Clostridia_Clostridiales_RuminococC

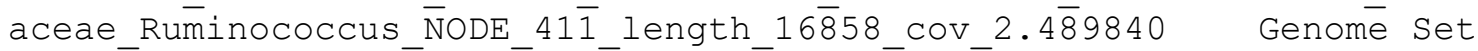

$16858 \mathrm{~N} / \mathrm{A} \quad \mathrm{IC} E$ Firmicutes

NZ_FCOY01000006.1_Xanthomonas_sp._SN8_genome_assembly_contig_contig0000 6_whole_genome_shotgun_sequence - Genome Set $^{-} 1618547$ - IncP ICE

Proteobacteria

20298_3_65_Firmicutes_Clostridia_Clostridiales_Lachnospiraceae_NA_.20298_ $365 . \overline{2} 7^{-}$Genome Set $33740 \mathrm{~N} / \mathrm{A}$ ICE Firmicutes

UMGGS901_SRR6028340_bin.15_Bacteroidetes_Bacteroidia_Bacteroidales_Bactero

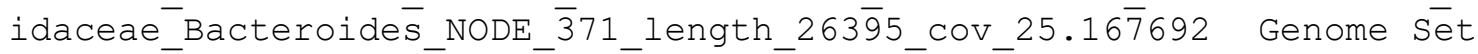

$2 \overline{6} 395 \mathrm{~N} / \mathrm{A} \quad \mathrm{ICE}$ Băcteroidetes

14207_7_9_Firmicutes_Clostridia_Clostridiales_Lachnospiraceae_Lachnospira _.14207_ 7 - 9.16 Genome Set $32 \overline{37}$ N/A N/A Firmicutes 
8080_1_78_Firmicutes_Clostridia_Clostridiales_Peptostreptococcaceae_Clost ridium_ERS̄138176.8080_1_78.7 Genome Set $26019 \overline{9} \quad$ N/A ICE Firmícutes UMGS170̄2 SRR5106295 bin.36 Firmicutes Clostridia Clostridiales NA NA NODE 64 length 122680 cōv $5.25 \overline{6} 813$ Genome set $12 \overline{2} 680$ N/A ICE

Firmicutes

13414_6_53_Firmicutes_Clostridia_Clostridiales_Ruminococcaceae_Faecalibac terium_.134 14 6 $53.17^{-}$Genome Set $56887 \mathrm{~N} / \mathrm{A}$ ICE Firmicutes

UMGS $47 \overline{2}$ ERR15 $\overline{7} \overline{6} \overline{19}$ bin. 63 Firmicutes Clostridia Clostridiales Lachnospira ceae_Ros̄eburia_NODE_153_lēength_92445_COV_49.202782 Genome Set 92445 N/A

ICE Firmicutes

UMGS971_ERR1620373_bin.39_Bacteroidetes_Bacteroidia_Bacteroidales_Porphyr omonadaceae_Butyricimonas_NoDE_1335_lenḡth_19849_cov__5.154643 Geñome Set $19849 \overline{\mathrm{N}} / \mathrm{A}$ Plasmid - Bacteröidetes

NZ GL891981.1 Dysgonomonas gadei ATCC BAA-

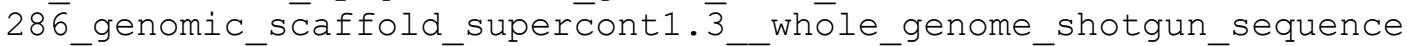

Genome set $467 \overline{0} 11 \quad$ N/A ICE Bacteroidetes

NZ_KQ236092.1_Parabacteroides_sp._2_1_7_genomic_scaffold_supercont2.1_wh olēegenome_shōtgun_sequence Genome $\overline{S e t}^{-}-15589-$ N/A ICE

Bacteroidetes

20287_6_53_Bacteroidetes_Bacteroidia_Bacteroidales_Bacteroidaceae_Bactero

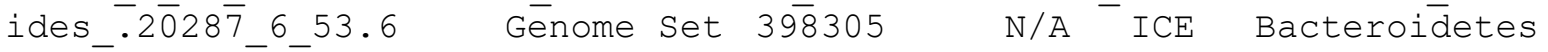
$1420 \overline{7}$ 6_3_Bācteroidetes_Bacteroidia_Bacteroidales_Porphyromonadaceae_NA_. 142076_3.32 Genome Set 23952 N/A N/A Bacteroidetes

NZ_LN $\overline{8} 6 \overline{5} 153.1$ Eubacterium_sp._SB2_genome_assembly_Eubacterium_massiliense scaffold scäffold00009 whole_genome_shotgun sequence Genome set
$2375 \overline{8} 04$
$\mathrm{N} / \mathrm{A}$
Firmicutes

14207_7_37_Firmicutes_Clostridia_Clostridiales_Lachnospiraceae_Dorea_.142 $077^{7} \overline{3} 7^{-} .19^{-}$Genome Set 80552 N/A ${ }^{-}$ICE Firmicutes

UMGGS 458 SRR5056748_bin.93_Firmicutes_Clostridia_Clostridiales_Eubacteriac eae_Eubācterium_NODE_6627_length_7350_cov_4.081014 Genome Set 7350 N/A

N/A Firmicutes

13470_2_58_Firmicutes_Clostridia_Clostridiales_NA_NA_.13470_2_58.9

Genome set $1706 \overline{7} 4 \quad$ N/A ${ }^{-}$ICE Firmicütes

NZ_GG705173.1_Bacteroides_sp._2_1_22_genomic_scaffold_supercont1.1_whole gēnome shotgünsequence Genome Set $1674 \overline{6} 41$ N/A ICE

Bacteroidetes

18048_2_85_Firmicutes_Clostridia_Clostridiales_Peptostreptococcaceae_Clos

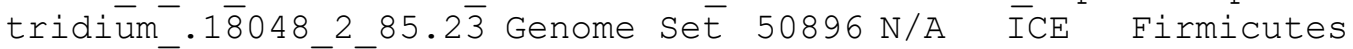

21673 4_87_Firmicutes_Clostridia_Clostridiales_Lachnospiraceae_Dorea_.216

$73487.13^{-}$Genome Set $53889 \mathrm{~N} / \mathrm{A}^{-}$ICE Firmicütes

14 $\overline{2} 0 \overline{7}$ 7_37_Firmicutes_Clostridia_Clostridiales_Lachnospiraceae_Dorea_.142

07 7_37.6- Genome Set 170289 - N/A ICE Firmicutes

NZ_AC $\bar{C} Y 01000004.1$ Roseburia_inulinivorans_DSM_16841_R_inulinivorans-

1.0.1_Cont4.1_whöle_genome_shotgun_sequeñce Genome $\overline{\text { Set }} 61552$ N/A ICE Firmicutes

20298_3_69_Firmicutes_Clostridia_Clostridiales_Ruminococcaceae_NA_.20298_ $369 . \overline{7}$ - Genome Set 223310 N/A ICE Firmicutes

UM̄GS122_SRR1779117_bin.2_Firmicutes_Bacilli_Bacillales_Paenibacillaceae_P

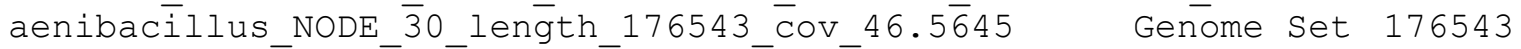

N/A ICE Firmicutes

21673_4_36_Firmicutes_Clostridia_Clostridiales_NA_NA_.21673_4_36.1

Genome set $8134 \overline{6} 5$ N/A ${ }^{-}$ICE Firmicutes 
NZ_DS981486.1_Bacteroides_coprocola_DSM_17136_Scfld_02_59_genomic_scaffol d_whole_genome_shotgun_seqquence Genome set $80142 \overline{\mathrm{N}} / \overline{\mathrm{A}} \overline{\mathrm{I}} \mathrm{CE}$

\section{Bacteroidetes}

18048267 Firmicutes Clostridia Clostridiales Lachnospiraceae Blautia.1 $8048 \overline{2} \overline{6} 7 . \overline{4}$ Genome Set $385361-$ N/A ICE Firmicutes

UMGS519_ERR1018206_bin.15_Actinobacteria_Actinobacteria_Coriobacteriales_ Coriobacteriaceae_Collinsēlla_NODE_679_lēngth_17298_cov_5.935800

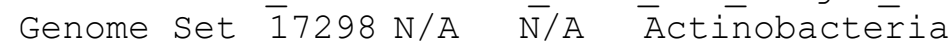

UMGS1188_SRR2155371_bin.11_Firmicutes_Clostridia_Clostridiales_NA_NA_NODE _35_length_110837_cov_11.6̄̄2636 Genome Set $11 \overline{0} 837$ N/A ICE

Firmicutes

NZ_HE978580.1_Peptostreptococcaceae_bacterium_ph1_genomic_scaffold_scaff

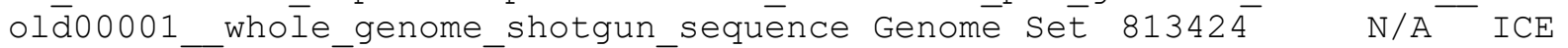

Firmicutes

UMGS1650 SRR585761 bin.1 Firmicutes Clostridia Clostridiales Peptostrepto coccaceae_Clostridium_NOD̄E_4_length_101668_cov_7.93269 Genome Set
$101 \overline{6} 68$
N/A N/A Firmicutes

UMGS1709_SRR1761698_bin.37_Firmicutes_Clostridia_Clostridiales_Lachnospir

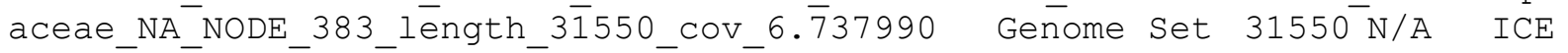
Firmicutes

21673_4_3_Firmicutes_Clostridia_Clostridiales_Lachnospiraceae_NA_.21673_4 3.2 Genome set $914 \overline{7} 67 \quad \mathrm{~N} / \overline{\mathrm{A}}$ ICE Firmicutes

UMGGS1069_SRR6028370_bin.24_Verrucomicrobia_Opitutae_Opitutales_NA_NA_NODE _8_length_169942_cov_10.467729 Genome set $16994 \overline{2}$ N/A ICE

Verrucomicrobia

UMGS493_SRR5279266_bin.8_Bacteroidetes_Bacteroidia_Bacteroidales_Prevotel laceae_Prevotella_NoDE_8 8 _length_57000_COV_38.1881- Genome Set $5 \overline{7} 000$ N/A

ICE Bacteroidetes

14207_7_89_Firmicutes_Clostridia_Clostridiales_NA_NA_.14207_7_89.31

Genome Set $3955 \overline{5}$ N/A ICE ${ }^{-}$Firmicutes

UMGS670_ERR1190964_bin.38_Firmicutes_Clostridia_Clostridiales_NA_NA_NODE_

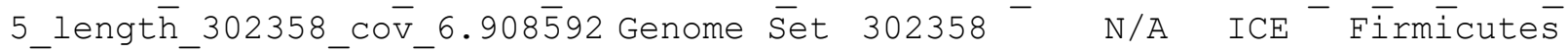

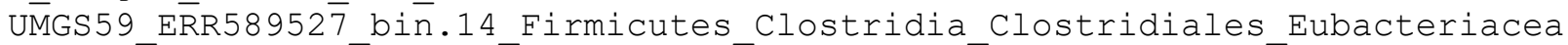

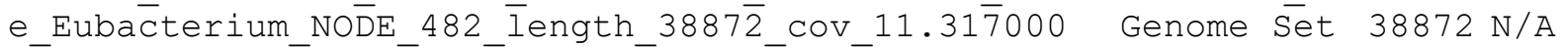

N/A Firmicutes

UMGS1292 ERR321194 bin.11 Bacteroidetes Bacteroidia Bacteroidales Rikenel laceae_Āistipes_NŌDE_1_lēength_282262_cov_8.46361 Genome Set $28 \overline{2} 262$

$\bar{N} / \mathrm{A} \quad \mathrm{ICE}$ Bacteroidetes

NZ_JH414767.1_Tannerella_sp._6_1_58FAA_CT1_genomic_scaffold_supercont1.2_ _whole_genome_shotgun_sequence ${ }^{-}$Genome Set $6958 \overline{11}$ N/A ICE Bacteroidetes

13470_2_92_Firmicutes_Clostridia_Clostridiales_Lachnospiraceae_NA_.13470_

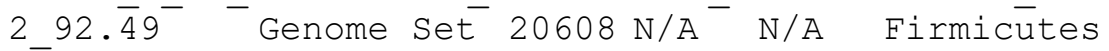

UMMGS1032_ERR414261_bin.23_Tenericutes_NA_NA_NA_NA_NODE_829_length_23068_C ov $5.813 \overline{9} 75$ Genome Set 23068 N/A Plasmid - Tenéricutes

NZ_DS499711.1_Clostridium_scindens_ATCC_35704_Scfld_02_33_genomic_scaffol d_whole_genome_shotgun_séquence Genome set 95416 N/A ICE Firmicutes UMGS1036_ERR011̄158_bin.10_Bacteroidetes_Bacteroidia_Bacteroidales_Prevote llaceae_Prevotella_NODE_1433_length_29192__Cov_6.5401-Genome Set $29 \overline{1} 92$ N/A

ICE Bacteroidetes

UMGS1005_SRR4408017_bin.22_Bacteroidetes_Bacteroidia_Bacteroidales_Porphy romonadac̄eae_Parabac̄teroides_NODE_6935_lēength_2246_cóv_7.700593

Genome Set 2246 N/A ICE Bacteroidetes 
NZ_JH379444.1_Prevotella_stercorea_DSM_18206_genomic_scaffold_Scfld979_w hole genome_shotgun sequēence Genome Set $5936 \overline{1}$ N/A ICE Bactēroidetes 14207_7_1_Fírmicutes_Clostridia_Clostridiales_Lachnospiraceae_Lachnospira $.142 \overline{0} \overline{7}^{-} 7^{-} 1.2$ Genome Set $46 \overline{1132}$ N/A N/A Firmicutes

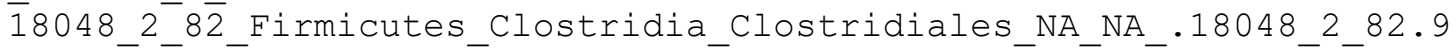

Genome set $8922 \overline{1}$ N/A ICE Firmicutes

13414_6_43_Firmicutes_Clostridia_Clostridiales_Ruminococcaceae_NA_.13414_ $643 . \overline{2}-$ Genome Set 673861 N/A ICE Firmicutes

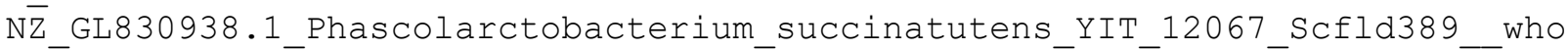
le_genome_shotgun_sequence Genome Set 76851 N/A ICE Firmicutes UMGGS96_SRR̄769526_bin.2_Firmicutes_Clostridia_Clostridiales_Lachnospiracea e_NA_NŌDE_236_leñgth_13506_cov_8.0̄5784 Genome set $13506 \mathrm{~N} / \mathrm{A}$ ICE

Firmicutes

UMGS800 ERR1293918 bin.21 Bacteroidetes Bacteroidia Bacteroidales Porphyr omonadaceae_NA_NODE_11_length_167619_cov_z22.900814 Genome set $16 \overline{7} 619$

N/A ICE Bacteroidetes

UMGS1038_ERR525948_bin.22_Tenericutes_Mollicutes_Erysipelotrichales_Erysi pelotrichaceae_Massiliomicrobiota_NODE_2035_length_7700_cov_4.8361

Genome Sēt 7700 N/A Plasmid - Tenēricutes

UMGS501_ERR1913117_bin.32_Bacteroidetes_Bacteroidia_Bacteroidales_Porphyr omonadaceae_NA_NODE_4_lenğth_275284_cov_10.250108 Genome Set $27 \overline{5} 284$

N/A ICE Bacteroidetés

13470_2_67_Bacteroidetes_Bacteroidia_Bacteroidales_Porphyromonadaceae_Par abactēeroides_.13470_2_67.7 Genome Set 301546 N/A ICE

Bacteroidetes

UMGS1628_SRR5056930_bin.3_Firmicutes_Clostridia_Clostridiales_Lachnospira ceae_NA_NODE_2272_lēength_1̄3761_COV_4.154677 Genome Set $1376 \overline{1}$ N/A ICE

Firmicutes

14207_7_54_Firmicutes_Clostridia_Clostridiales_NA_NA_.14207_7_54.13

Genome set $6533 \overline{1}$ N/A ICE Firmicutes

12718_7_47_Firmicutes_Clostridia_Clostridiales_NA_NA_.12718_7_47.2

Genome Set $4908 \overline{4} 6 \quad$ N/A ${ }^{-}$ICE Firmicutes

UMGS329_SRR6028346_bin.15_Bacteroidetes_Bacteroidia_Bacteroidales_NA_NA_N

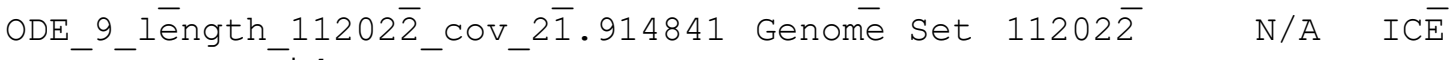

Bacteroidetes

UMGS1440_SRR1761678_bin.28_Firmicutes_Clostridia_Clostridiales_Peptostrep tococcacēae_Clostrī̄ium_Nō̄EE_2_length_356471_cov_49.212914 Genome Set $35647 \overline{1} \quad$ N/A ICE Firmicutes

14207_7_89_Firmicutes_Clostridia_Clostridiales_NA_NA_.14207_7_89.16

Genome set $7870 \overline{1}$ N/A ICE ${ }^{-}$Firmicutes

UMGS144_SRR3737013_bin.8_Proteobacteria_Gammaproteobacteria_Aeromonadales _Succinivibrionaceàe_Succinatimonas_NODE_72_length_108762_Cov_41.565603
Genome Set $108 \overline{7} 62$
$\mathrm{N} / \mathrm{A}$
N/A Proteobactēria

13414_6_47_Firmicutes_Clostridia_Clostridiales_Lachnospiraceae_Lachnobact

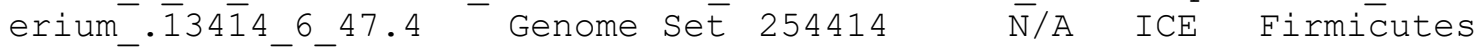

UMGS5 $\overline{6} 8$ ERR1 $\overline{3} 0 \overline{5} 892$ bin.4_Firmicutes_Clostridia_Clostridiales_NA_NA_NODE_3 65_length_54717_cov_69.941093 Genome set 54717 N/A ICE Ē Firmicutēs UMḠS505_ERRR12937̄07_bin.3_Firmicutes_Clostridia_Clostridiales_Ruminococcac

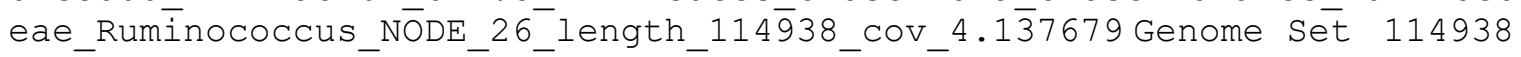

N/A ICE Firmicutes

20287_6_9_Bacteroidetes_Bacteroidia_Bacteroidales_Bacteroidaceae_Bacteroi des_. $20 \overline{2} 8 \overline{7} \_6 \_9.11$ Genome ${ }^{-}$Set $193709^{-}$N/A ICE $^{-}$Bacteroidetes 
UMGS781_ERR1305892_bin.56_Proteobacteria_Deltaproteobacteria_Desulfovibri

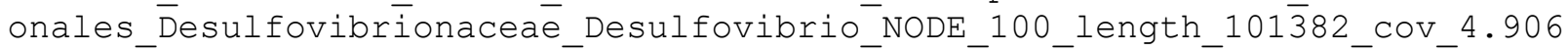
876 Genome set 101382 - N/A ICE Proteōbacteria UMGS1989 ERR1190931 bin.4 Firmicutes Clostridia Clostridiales NA NA NODE 1474 lenḡth 21870 Cov $3.2 \overline{2} 6496$ Geñome set $2 \overline{1} 870$ N/A ICE Firmícutes

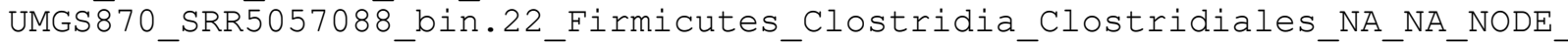
112_lenḡth_85141_cov_8.498895 Geñome set $8 \overline{5141}$ N/A ICE Firmícutes NZ_GL892010.1_Dysgonomonas_mossii_DSM_22836_genomic_scaffold_supercont1.7 whole_genoméshotgun_sequence Genome Set $18261 \overline{7}$ N/A ICE Bäcteroidetes

UMGS40_SRR2912796_bin.41_Firmicutes_Clostridia_Clostridiales_Ruminococcac eae_Ruminococcus_NoDE_24_length_141787_COV_18.9̄92257 Genome Set $141787 \quad$ N/A I $\bar{C} E$ Firmicutes

UMGS253 ERR1293894 bin.25 Firmicutes Clostridia Clostridiales Ruminococca ceae_Faēcalibacterīum_NODE_160_length̄58565_cov_12.603418Genome Set $58565 \mathrm{~N} / \mathrm{A} \quad \mathrm{ICE}$ Firmicutes

13414_6_37_Firmicutes_Clostridia_Clostridiales_NA_NA_.13414_6_37.4 Genome Set $1499 \overline{7} 8$ N/A ICE Firmicutes

NZ_HE978611.1_Brevibacterium_sp._JC43_genomic_scaffold_scaffold00012_wh olèe_genome_shötgun_sequence Genome Set 62517 N/A Plasmid Actiñobactería

UMGS2042_SRR341641_bin.4_Bacteroidetes_Bacteroidia_Bacteroidales_Bacteroi daceae_Bacteroides_NODE_490_length_17164_Cov_2.2363i1 Genome Set $\overline{17} 164 \mathrm{~N} / \mathrm{A} \quad \mathrm{I} \overline{\mathrm{C}} \mathrm{E}$ Bactēroidetes

UMGS1097_ERR525737_bin.51_Firmicutes_Clostridia_Clostridiales_Lachnospira

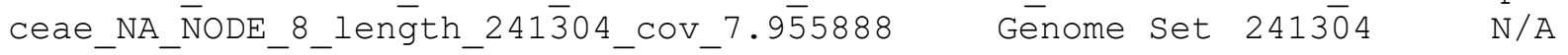
ICE Firmicutes

UMGS 859 ERR 414316 bin.12_Proteobacteria_Alphaproteobacteria_Rickettsiales _NA_NA_NODE_14_length_225374_COV_40.173319 Genome set $22 \overline{5} 374$ N/A ICE Proteobacteria

UMGS365_SRR5558121_bin.15_Firmicutes_Clostridia_Clostridiales_NA_NA_NODE_ 98_length_115411_cō_8.770̄944 Geñome set $1 \overline{15411}$ N/A ${ }_{\text {I }} \overline{\mathrm{CE}}$ Firmicutes

UMGS1399_SRR4408050_bin.11_Bacteroidetes_Bacteroidia_Bacteroidales_Bacter

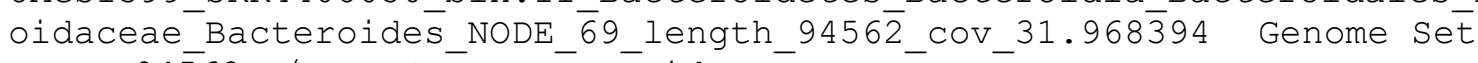
$94 \overline{5} 62 \mathrm{~N} / \mathrm{A} \quad \mathrm{ICE}^{-}$Bacteroidetes

UMGS1132_ERR1620269_bin.14_Firmicutes_Clostridia_Clostridiales_NA_NA_NODE 355_lenğth_34204_Cov_7.385926 Genome Set $34 \overline{204}$ N/A ICE Firmicutes

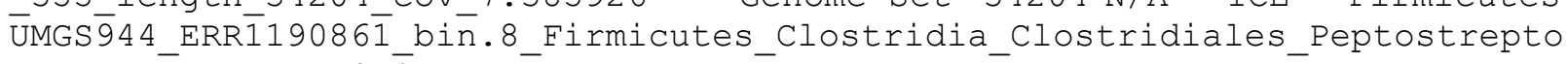

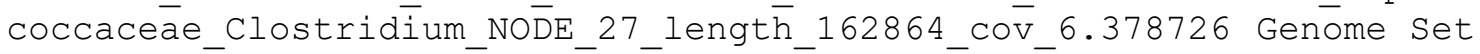
$162864 \quad$ N/A ${ }_{\text {ICE }}{ }^{2}$ Firmicutes

20298_3_4_Proteobacteria_Gammaproteobacteria_Enterobacteriales_Enterobact eriacéae_citrobacter_.20298_3_4.9 Genome Set 149896 IncFI $\bar{I}$ Plāsmid Proteobactēéia

21673_4_33_Bacteroidetes_Bacteroidia_Bacteroidales_Porphyromonadaceae_Par abactēröidés_.21673_4_33.7 Genome set $269567-$ N/A ICE Bacteroidetes

NZ_HG726020.1_Bacteroides_neonati_strain_MS4_whole_genome_shotgun_sequen ce Genome Set $1480366^{-}$N/A ICE Bacteroidetes 21673_4_19_Firmicutes_Clostridia_Clostridiales_Lachnospiraceae_Blautia_.2 1673_4 $\overline{1} 9 . \overline{9}$ Genome Set 175874 N/A ICE Firmicutes NZ_KI $9 \overline{6} 5381.1$ Fusobacterium_nucleatum_13_3C_genomic_scaffold_adlCxsupercont1.1_whole_genome_shotgun_sequence Genome set 1700382 N/A ICE Fusobacteria 
12718_7_89_Actinobacteria_Actinobacteria_Coriobacteriales_Coriobacteriace

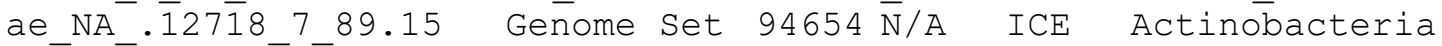
UMGGS1450 ERR $\overline{2} 2 \overline{1} 427$ bin. 5 Bacteroidetes Bacteroidia Bacteroidales Bacteroi daceae Bācteroides NODE 668 length $884 \overline{5} \mathrm{COV} 2.1856 \overline{7}$ Genome set $8 \overline{8} 45$ N/A

$\overline{\mathrm{N}} / \mathrm{A} \quad$ Bacteroidetes

UMGS639_DRR042328_bin.21_Actinobacteria_Actinobacteria_Coriobacteriales_C oriobactétiaceae_Collinsēlla_NODE_110_lēngth_130575_cō̄ 20.622194

Genome Set $130575 \quad \bar{N} / \mathrm{A}{ }^{-}{ }_{\mathrm{ICE}}^{-}$Actiñobactēria

UMGS1502_SRR3992965_bin.54_Actinobacteria_Actinobacteria_Coriobacteriales _Coriobacteriaceae_NA_NODE_4333_length_10732_cov_3.84082 $\overline{6}$ Genome Set

$10732 \mathrm{~N} / \mathrm{A}$ Pİasmid - Actinobacteria

UMGS219_ERR321453 bin.7 Bacteroidetes Bacteroidia_Bacteroidales Bacteroid aceae Bäcteroides NODE $\overline{1}$ length $12762 \overline{6}$ cov $3.5547 \overline{8}$ Genome set $\overline{1} 27626$

N/A ICE Bacterōidetes

21673_4_87_Firmicutes_Clostridia_Clostridiales_Lachnospiraceae_Dorea_.216 734 87.7 Genome Set 131246 N/A ICE Firmicutes

UMGGST1436 ERR1190898 bin.13 Firmicutes Clostridia Clostridiales Lachnospir

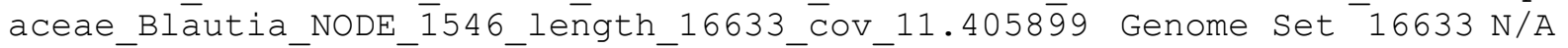

ICE Firmicutes

18048_1_72_Firmicutes_Clostridia_Clostridiales_Lachnospiraceae_Blautia_.1 $8048 \overline{1} \overline{7} 2 . \overline{8}$ Genome Set $195242-$ N/A ICE Firmicutes

$2167 \overline{3} \overline{4}$ 19_Firmicutes_Clostridia_Clostridiales_Lachnospiraceae_Blautia_.2 1673 4 $\overline{1} 9 . \overline{2} 0$ Genome set $702 \overline{11}$ N/A ICE Firmicutes

UMGS40_SRR2912796_bin.41_Firmicutes_Clostridia_Clostridiales_Ruminococcac

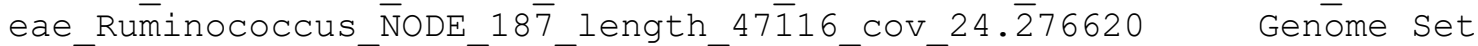

$$
47116 \mathrm{~N} / \mathrm{A}-\mathrm{N} / \mathrm{A}-\mathrm{Fi} \overline{\mathrm{rm}} \mathrm{icutes}
$$

NZ_GG704863.1_Enterobacter_cancerogenus_ATCC_35316_Scfld0_whole_genome_s hotgun sequence Genome Sēt 2235134 N/A ICE Proteobacteria 20287_6_88_Firmicutes_Clostridia_Clostridiales_Lachnospiraceae_NA_.20287_ $688 . \overline{1} 5^{-}$- Genome Set $133211-$ N/A ICE Firmicutes

NZ_GL636544.1_Sutterella_wadsworthensis_3_1_45B_genomic_scaffold_supercon t1.5_whole_genome_shotgün_sequence Gēome Set $226 \overline{673}$ N/A N/A

Proteobactería

UMGS486_ERR911961_bin.4_Firmicutes_Clostridia_Clostridiales_NA_NA_NODE_28 9 length 91142 Cov $22.4 \overline{1} 8775$ Genome Set 91142 N/A ICE Firmicutés

13414_6_53_Firmicutes_Clostridia_Clostridiales_Ruminococcaceae_Faecalibac terium . $13 \overline{4} 14653.31^{-}$Genome Set 11580 N/A ICE Firmicutes

UMGS $24 \overline{7}$ ERR32 $\overline{1} 4 \overline{7} 0$ bin.7_Bacteroidetes_Bacteroidia_Bacteroidales_Porphyrom onadaceāe_NA_NODE_3_lenḡth_200868_cov_14.7962 Genome set $200868^{-}$N/A

ICE $\overline{\text { Bacteroidetes }}$

UMGS83_ERR911953_bin.80_Firmicutes_Clostridia_Clostridiales_NA_NA_NODE_5 length $319042 \mathrm{COV}$ 13.719̄537 Genome Set $31904 \overline{2}$ N/A ICE E Firmicutes

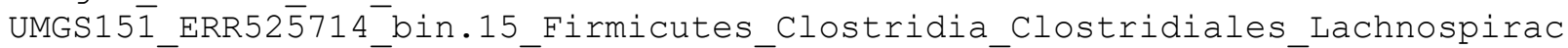

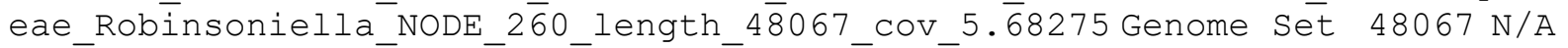
ICE Firmicutes

UMGS315_SRR5127596_bin.10_Actinobacteria_Actinobacteria_Coriobacteriales_ Coriobacteriaceae_Collinsēlla_NODE_1_length_138453_cov_1̄0.931422

Genome Set $\overline{1} 38453 \quad \bar{N} / \mathrm{A} \quad \overline{\mathrm{I}} \overline{\mathrm{CE}}$ Actinobactēria

13414_6_50_Firmicutes_Clostridia_Clostridiales_Lachnospiraceae_Dorea_.134 14 6_50.8- Genome Set 145632 N/A ICE Firmicutes

NZ_JḦ376421.1_Clostridium_citroniae_WAL-

17108_genomic_scaffold_supercont1.2_whole_genome_shotgun_sequence Genome set $69445 \overline{2} \quad$ N/A ICE Firmicutes 
21673_4_71_Firmicutes_Clostridia_Clostridiales_Peptostreptococcaceae_Clos tridium_.21673_471.6 Genome Set 152027 N/A ICE Firmicutes UMGS2027 SRR17 $\overline{6} 1 \overline{7} 21$ bin.40 Bacteroidetes Bacteroidia Bacteroidales Prevot

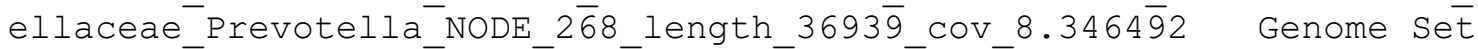

$36 \overline{9} 39 \mathrm{~N} / \mathrm{A} \quad \mathrm{ICE} \quad$ Bäcteroidetes

UMGS1003_ERR525813 bin.62 Bacteroidetes Bacteroidia_Bacteroidales Prevote

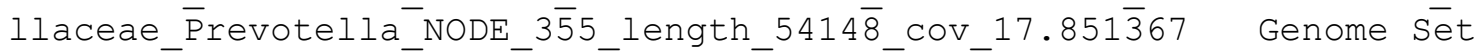

$5 \overline{4} 148 \mathrm{~N} / \mathrm{A} \quad \mathrm{I} \overline{\mathrm{C}} \mathrm{E}$ Bacteroidetes

NZ_JH992945.1_Bacteroides_oleiciplenus_YIT_12058_genomic_scaffold_superco

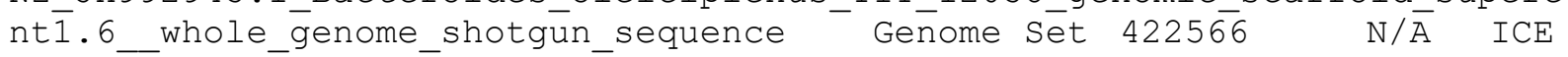

Bacteroidetes

UMGS709_SRR2992911_bin.12_Firmicutes_Clostridia_Clostridiales_Oscillospir

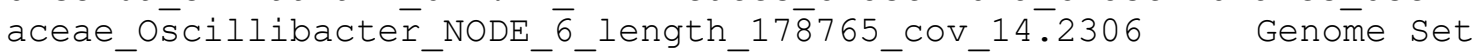

$178765 \quad \mathrm{~N} / \overline{\mathrm{A}} \quad \mathrm{ICE}-$ Firmicutes

UMGS568_ERR1305892_bin.4 Firmicutes_Clostridia_Clostridiales_NA_NA_NODE_8 2_length_109425_cov_66.307177 Genome Set $\overline{109425}$ N/A $\overline{\text { ICE }}$

Firmicutes

UMGS1201_ERR1620325_bin.78_Bacteroidetes_Bacteroidia_Bacteroidales_Porphy romonadaceae_Parabacteroidès_NODE_1052_lēength_24300_cov_5.163374

Genome Set 24300 N/A ICE Bacteroidetés

UMGS767_ERR1190550_bin.24_Proteobacteria_Betaproteobacteria_Burkholderial es_Suttēerellaceae_sutterē̄la_NODE_502_length_34214_cov_9.469891

Genome Set 34214 N/A N/A Proteobacteria

21673_4_3_Firmicutes_Clostridia_Clostridiales_Lachnospiraceae_NA_.21673_4 3.3 Genome set $282 \overline{0} 87 \quad$ N/A $\quad$ ICE Firmicutes

21673_4_71_Firmicutes_Clostridia_Clostridiales_Peptostreptococcaceae_Clos

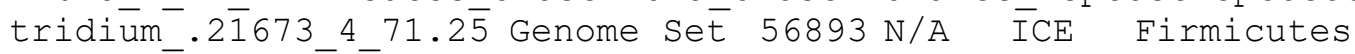

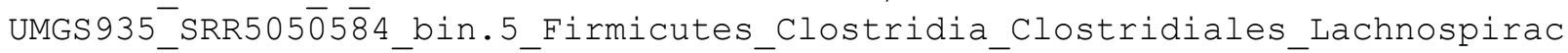

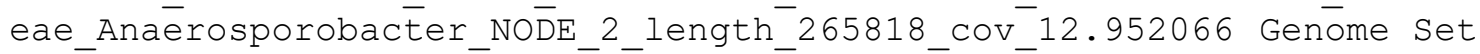

$265818 \quad$ N/A ICE Firmicutes

13414_6_17_Firmicutes_Clostridia_Clostridiales_Lachnospiraceae_Ruminococc us. $1 \overline{3} 4 \overline{1} 4 \overline{6} 17.1$ Genome Set $497 \overline{25} 2$ N/A $\overline{\text { ICE Firmicutes }}$

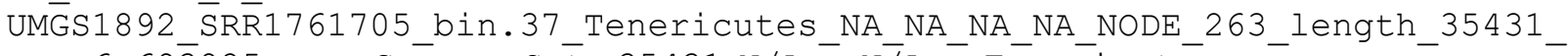

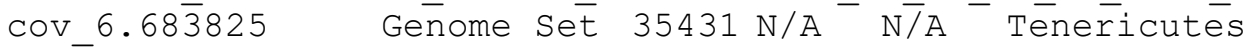

UMGS̄I115_SRR3992981_bin.40_Firmicutes_Clostridia_Clostridiales_NA_NA_NODE 2512_leñgth_13477_cov_10.5̄75746 Genome set $13 \overline{4} 77$ N/A ICE Firmicutes 12718_7_73_Firmicutes_C̄lostridia_Clostridiales_Lachnospiraceae_NA_.12718_ $773 . \overline{1}$ - Genome set 1695398 N/A ICE Firmicutes

20287_6_37_Firmicutes_Clostridia_Clostridiales_NA_NA_.20287_6_37.27

Genome Set $3850 \overline{3}$ N/A ICE Firmicutes

NZ_DS483492.1_Faecalibacterium_prausnitzii_M21_2_Scfld_02_13_genomic_scaf fold_whole_genome_shotgun_sequence Genome ${ }^{-} \bar{e} \overline{e t} 47 \overline{391} \overline{\mathrm{N}} / \mathrm{A}^{-}$ICE Firmicutes

UMGS233_SRR6028305_bin.9_Firmicutes_Clostridia_Clostridiales_Ruminococcac eae_NA_NoDE_111_length_37̄121_COV_14.159823 Genome set $371 \overline{2} 1 \mathrm{~N} / \mathrm{A}$ ICE Firmicutes

UMGS229_ERR414321_bin.1_Firmicutes_Clostridia_Clostridiales_Peptostreptoc

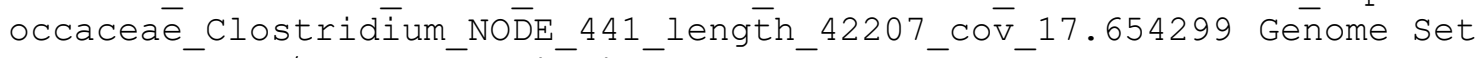
$42 \overline{2} 07 \mathrm{~N} / \mathrm{A} \quad \mathrm{ICE}^{-}$Firmicutes

13470_2_81_Firmicutes_Clostridia_Clostridiales_Lachnospiraceae_Blautia_.1 $3470 \overline{2} \overline{8} 1 . \overline{1} 4$ Genome set $863 \overline{12} \mathrm{~N} / \mathrm{A}$ ICE Firmicutes UMGS2 $8 \overline{2}$ _ERR525699_bin.23_Bacteroidetes_Bacteroidia_Bacteroidales_Porphyro monadacēae_Parabac̄teroidēs_NODE_241_length_62404_cōv_5.943191 Gēnome Set $6240 \overline{4} \mathrm{~N} / \mathrm{A} \quad$ ICE Bacteroì̄etes 
UMGS1469_ERR688640_bin.60_Tenericutes_Mollicutes_Erysipelotrichales_Erysi pelotrichaceae_Solōbacterīum_NoDE_494_length_4667̄3_cov_6.660775

Genome Set 46673 N/A Plasmid - Tenericutes

14207_7_43_Firmicutes_Clostridia_Clostridiales_Lachnospiraceae_NA_.14207_ $743 . \overline{1}-$ Genome Set 481317 - N/A ICE Firmicutes

13̄470_2_81_Firmicutes_Clostridia_Clostridiales_Lachnospiraceae_Blautia_.1 $3470 \overline{2} \overline{8} 1 . \overline{7}$ Genome Set $132173-$ N/A ICE Firmicutes

NZ_LTT575475.1_Paenibacillus_sp._Marseille-

P2 $\overline{4} 72$ _whole_ḡenome_shotgun_sequence Genome set 34735 N/A N/A Firmicutes

13414_6_17_Firmicutes_Clostridia_Clostridiales_Lachnospiraceae_Ruminococc us_. $1 \overline{3} 4 \overline{1} 4 \bar{\sigma}_{17.5}$ Genome set $182 \overline{4} 75 \quad \mathrm{~N} / \mathrm{A} \quad \overline{\mathrm{IC}} \mathrm{E}$ Firmicutes

NZ_HE978582._._Peptostreptococcaceae_bacterium_ph1_genomic_scaffold_scaff

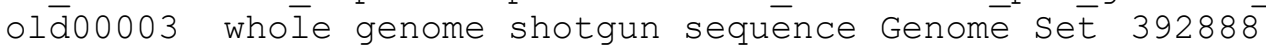

rep_cluster_1663 ICE Firmicutes

20298_3_81_Firmicutes_Clostridia_Clostridiales_Lachnospiraceae_Blautia_.2 $0298 \overline{3} \overline{8} 1 . \overline{1} 0 \quad$ Genome Set $132 \overline{354} \quad$ N/A ICE Firmicutes

UMGST772_ERR321392_bin.13_Tenericutes_NA_NA_NA_NA_NODE_63_length_25294_COV

$2.5733 \overline{2}$ Genome Set $2 \overline{5} 294 \mathrm{~N} / \mathrm{A}$ N/A Tenericutes

21673_4_71 Firmicutes_Clostridia_Clostridiales_Peptostreptococcaceae_Clos tridium_.21673_471.9 Genome Set 112035 N/A ICE Firmicutes

UMGS637_SRR527 $\overline{5} 4 \overline{5} 4$ bin.27 Firmicutes_Clostridia_Clostridiales Ruminococca ceae_NA_NODE_2010_Iength_6543_cov_15.7981 Genome set $6543^{-}$N/A N/A

Firmicutes

UMGS39 ERR525754 bin.18 Firmicutes Clostridia Clostridiales Eubacteriacea

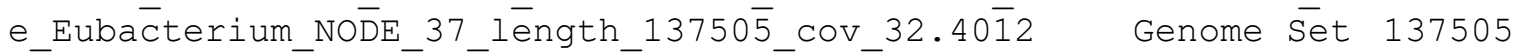
N/A ICE Firmicutes

NZ_FMIZ01000005.1_Ruminococcus_sp._Marseille-

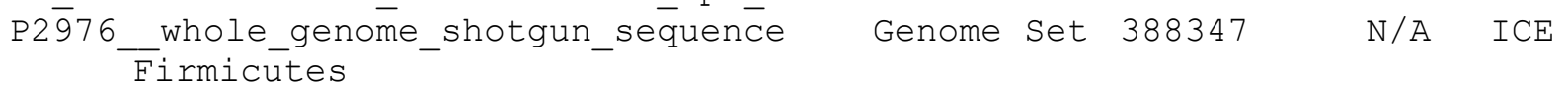

UMGS1730_SRR5275432_bin.16_Firmicutes_Clostridia_Clostridiales_NA_NA_NODE _8988_length_2871_cov_5.359̄020 Genome set $28 \overline{71}$ N/A Plasmìd

Firmicutes

NZ_FCOY01000004.1_Xanthomonas_sp._SN8_genome_assembly_contig_contig0000 4_whole_genome_shotgun_sequence Genome Set 131009 N/A ICE

Proteobacteria

UMGS156_SRR5558393_bin.8_Bacteroidetes_Bacteroidia_Bacteroidales_Prevotel laceae_Prevotella_NoDE_295_length_33189_COV_8.602682 Genome Set

$\overline{3} 3189 \mathrm{~N} / \mathrm{A}$ ICE Bacteroidē̄es

UMGS1869_ERR414339_bin.30_Bacteroidetes_Bacteroidia_Bacteroidales_Bactero idaceae_Bacteroides__NODE_T1424_length_22052_cov_5.617 675 Genome Set

$2 \overline{2} 052 \mathrm{~N} / \mathrm{A} \quad \mathrm{ICE}$ Bacteröidetes

NZ_AKCB01000003.1_Coprobacillus_sp._D6_cont1.3_whole_genome_shotgun_sequ ence Genome Set $\overline{3} 97244 \quad$ N/A $\overline{\text { ICE }}$ Tenericutes

13414_6_50_Firmicutes_Clostridia_Clostridiales_Lachnospiraceae_Dorea_.134 $146 \overline{50.1}$ - Genome Set 424995 N/A ICE Firmicutes

$14 \overline{2} 0 \overline{7} 7$ 75 Firmicutes_Clostridia_Clostridiales_Ruminococcaceae_Ruminococc us_.1 $\overline{4} 2 \overline{0} 7 \overline{7}[57.1$ Genome Set $333 \overline{975}$ N/A ICE Firmicutes

NZ_CBYL010000041.1_Clostridium_jeddahense_strain_JCD_whole_genome_shotgu n_sequence Genome Set 32365 N/A N/A Firmicutēs

UMMGS1433_SRR6028647_bin.11_Bacteroidetes_Bacteroidia_Bacteroidales_Prevot

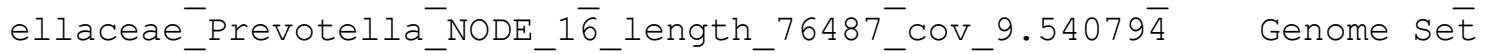

$76 \overline{4} 87 \mathrm{~N} / \mathrm{A} \quad \mathrm{ICE} \quad$ Bäctēeroidetēes 
UMGS1402_ERR209831_bin.1_Tenericutes_NA_NA_NA_NA_NODE_3_length_87560_COV_ 5.002148 Genome Set $8 \overline{7} 560 \mathrm{~N} / \mathrm{A} \quad \mathrm{N} / \overline{\mathrm{A}}$ Teñericutes

UMGS35_ERR525726_bin.20_Firmicutes_Clostridia_Clostridiales_Peptostreptoc

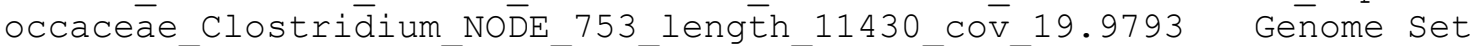
$11 \overline{4} 30 \mathrm{~N} / \mathrm{A} \quad \mathrm{N} / \mathrm{A}^{-}$Firmicūtes

UMGS1681_ERR1620362_bin.35_Firmicutes_Clostridia_Clostridiales_Peptostrep

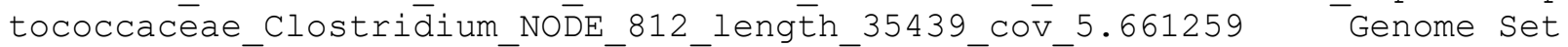

$35439 \overline{\mathrm{N}} / \mathrm{A} \quad \mathrm{N} / \mathrm{A} \quad \overline{\mathrm{F}} \mathrm{irmi} \overline{\mathrm{cut}} \overline{\mathrm{s}}$

21673_4_36_Firmicutes_Clostridia_Clostridiales_NA_NA_.21673_4_36.5

Genome Set $1786 \overline{5} 1 \quad$ N/A ${ }^{-}$ICE Firmicutes

13414_6_39_Firmicutes_Clostridia_Clostridiales_Ruminococcaceae_NA_.13414 6_39. $\overline{2}^{-}$- Genome Set 469987 - N/A ICE Firmicutes

UMMGS1208 ERR589513 bin.7 Bacteroidetes Bacteroidia Bacteroidales Prevotel laceae Prevotella NODE $1 \overline{7} 31$ length $182 \overline{4} 8 \mathrm{cov} 5.069 \overline{4} 77$ Genome Set

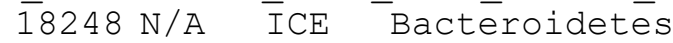

UMGS998 ERR1620304 bin.12 Proteobacteria Deltaproteobacteria Desulfovibri onales_Desulfovibrionaceaé_Desulfovibrio_NODE_209_length_48038_cov_5.5030 32 Genome set $48038 \mathrm{~N} / \mathrm{A}^{-}$ICE Proteōbacteria

14207_7_43_Firmicutes_Clostridia_Clostridiales_Lachnospiraceae_NA_.14207_ $743 . \overline{3} 0^{-}{ }^{-}$Genome Set $41454 \mathrm{~N} / \mathrm{A}^{-}$ICE Firmicütes

UM̄GS514_ERR1620277_bin.40_Actinobacteria_Actinobacteria_Coriobacteriales_ Coriobaçteriaceae_C̄ollinsēlla_NODE_478_lēngth_38943_cov_4.130966

Genome set $38943 \mathrm{~N} / \mathrm{A} \quad \overline{\mathrm{ICE}} \overline{\mathrm{A}} \mathrm{ctin}$ obacteria

UMGS160 SRR2992962 bin.4 Firmicutes Clostridia Clostridiales Ruminococcac

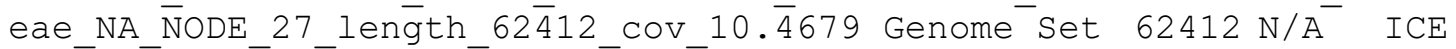

Firmicutess

UMGS1513_SRR5275432_bin.5_Tenericutes_NA_NA_NA_NA_NODE_22_length_179350_C ov 59.0833399 Geñome Sét $179350-\bar{N}^{-}{ }^{-} \overline{I C E}^{-}$Tenericutes

UMGGS192_ERR1018298_bin.4_Firmicutes_Clostridia_Clostridiales_Eubacteriace

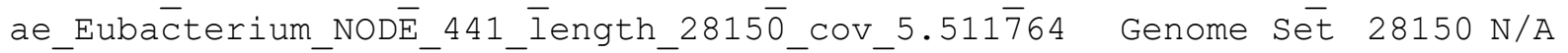

ICE Firmicutes

11861_6_65_Firmicutes_Clostridia_Clostridiales_Ruminococcaceae_NA_.11861_ 6 65.28 ${ }^{-}$Genome Set 34462 N/A ${ }^{-}$N/A Firmicütes

UMMGS1205 ERR209515 bin.8 Bacteroidetes Bacteroidia Bacteroidales Bacteroi

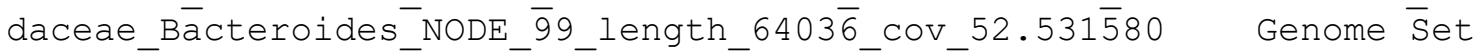

$\overline{6} 4036 \mathrm{~N} / \mathrm{A} \quad \mathrm{IC} E$ Bacteroidetes

UMGS261_ERR1190811_bin.41_Firmicutes_Clostridia_Clostridiales_NA_NA_NODE_

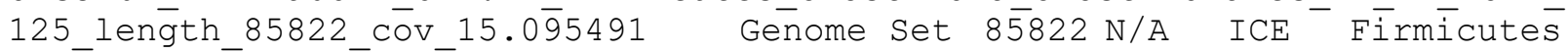
12718_7_72_Firmicutes_Clostridia_Clostridiales_NA_NA_.12718_7_72.7

Genome Set $1730 \overline{79}$ N/A ICE Firmicutes

NZ_FLKP01000002.1_Christensenella_timonensis_strain_Marseille-

P2 437 whole_genome_shotgun_sequeñee Genome set 2314156 N/A ICE Firmicutes

UMGS934_ERR1912962_bin.10_Firmicutes_Clostridia_Clostridiales_NA_NA_NODE_

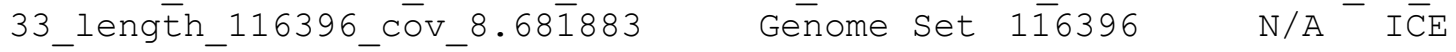

Firmicutes

UMGS1734_ERR688506_bin.12_Firmicutes_Clostridia_Clostridiales_Peptostrept

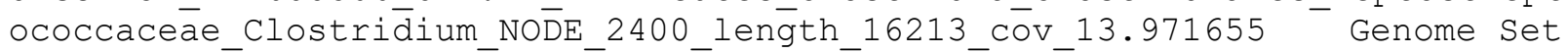
$1621 \overline{3} \mathrm{~N} / \mathrm{A}$ Plasmid $\overline{\mathrm{A}}$ - Firmicutes

NZ_DS995474.1_Collinsella_stercoris_DSM_13279_Scfld1_genomic_scaffold_wh olēe_genome_shōtgun_sequence Genome Set $81351 \overline{7} \quad \overline{\mathrm{N}} / \mathrm{A} \quad \mathrm{ICE}$ Actinobacteria 
NZ_DS990266.1_Clostridiales_bacterium_1_7_47_FAA_supercont2.7_genomic_sca ffōld_whole_ḡenome_shotgun_sequence - Génome sēt 55423 N/A ICE Firmicutes

21673471 Firmicutes Clostridia Clostridiales Peptostreptococcaceae Clos tridium_.21673_4_71.20 Genome Set 74436 N/A N $/ A$ Firmicutes

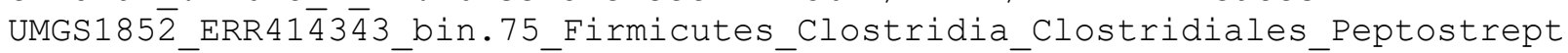

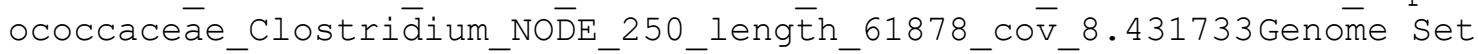

$6187 \overline{8} \mathrm{~N} / \mathrm{A} \quad \mathrm{N} / \mathrm{A}$ Firmicutes

20298_3_39_Firmicutes_Clostridia_Clostridiales_NA_NA_.20298_3_39.30

Genome Set $1800 \overline{4}$ N/A N/A Firmicutes

NZ_KB976103.1_Butyricicoccus_pullicaecorum_1.2_genomic_scaffold_acBRa-

supercont1.1_whole_genome_shotgun_sequencē Genome Sēt $186840 \overline{9}$ N/A ICE Firmicutes

UMGS1772_SRR341608_bin.22_Firmicutes_Clostridia_Clostridiales_Lachnospira

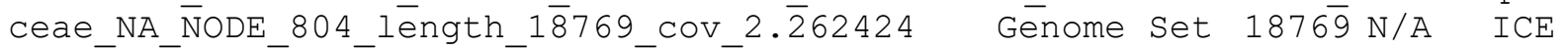

Firmicutes

UMGS218_SRR5580022_bin.2_Bacteroidetes_Bacteroidia_Bacteroidales_Bacteroi daceae_Bacteroides_NODE_627_length_12530_cov_12.007468 Genome Set

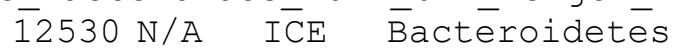

UMGS1730_SRR5275432_bin.16_Firmicutes_Clostridia_Clostridiales_NA_NA_NODE

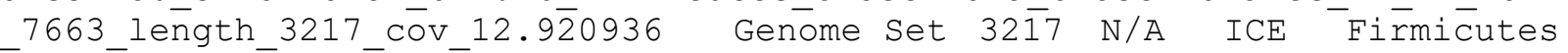
13470_2_62_Bācterōidē̄es_Bacteroidia_Bacteroidales_Bacteroidaceae_Bactero ides_.13 $47 \overline{0} \_2$ 262.1 Genome set $71 \overline{4} 338$ N/A ICE Bacteroidetes 21673_4_77_Firmicutes_Clostridia_Clostridiales_Peptostreptococcaceae_Clos tridium_.21673_477.3- Genome Set 732612 N/A ICE Firmicutes

UMGS302_ERR68850̄5_bin.28_Actinobacteria_Actinobacteria_Coriobacteriales_C oriobacteriaceae_Collinsēlla_NODE_9_length_179201_Cov_ō.282518 Genome Sēt $179201 \quad$ N/A ICE Actiñobacteria

NZ_ACII02000003.1_Ruminococcus_sp._5_1_39BFAA_acpfZ-

supercont2.3_whole_genome_shotgun_sequence Genome set 3112511 N/A ICE Firmicutes

UMGS1659_ERR1293936_bin.10_Firmicutes_Clostridia_Clostridiales_Lachnospir

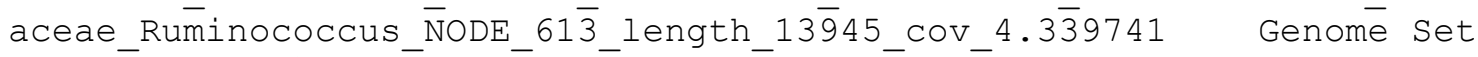
$13945 \mathrm{~N} / \mathrm{A} \quad \mathrm{N} / \mathrm{A}$ Firmicutes

NZ_DS264263.1_Eubacterium_ventriosum_ATCC_27560_Scfld021_genomic_scaffold whole_genomé_shotgun_sequence Genome Set $2 \overline{5} 26$ N/A Plasmid Firmicutès

18048_1_69_Firmicutes_Clostridia_Clostridiales_Lachnospiraceae_NA_.18048_ 1_69.17 - Genome Set $77872 \mathrm{~N} / \mathrm{A}$ ICE Firmicutes

Nz̄_CVPE01000004.1_Bacillaceae_bacterium_mt6_genome_assembly_Rubeoparvulum _mássiliensis_coñtig_contig00004_wholèegenome_shōtgun_sequence

Genome Set $5351 \overline{1} 6$ N/A ICE Firmicutes

UMGS218_SRR5580022_bin.2_Bacteroidetes_Bacteroidia_Bacteroidales_Bacteroi daceae_Bacteroides_NODE_7_length_147301_COV_6.314786 Genome Set $147301 \quad \mathrm{~N} / \mathrm{A} \quad \overline{\mathrm{I}} \overline{\mathrm{E}}$ Bacteroidetes

13470_2_93_Firmicutes_Clostridia_Clostridiales_Lachnospiraceae_NA_.13470_ 2.93.29 Genome Set $37545 \mathrm{~N} / \mathrm{A}$ ICE Firmicutes 13470_2_92_Firmicutes_Clostridia_Clostridiales_Lachnospiraceae_NA_.13470_ 2_92.18- Genome Set 92661 N/A ICE Firmicütes NZ__JH815242.1_Ralstonia_sp._5_2_56FAA_genomic_scaffold_supercont2.7_whol e_genome_shotgun_sequence - Genome set 36381 rep_cluster_1864 Plasmid Proteobacteria 
UMGS37_ERR1578662_bin.38_Firmicutes_Clostridia_Clostridiales_Lachnospirac

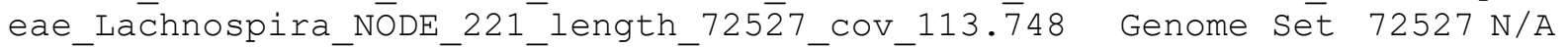
ICE Firmicutes

20287_6_88_Firmicutes_Clostridia_Clostridiales_Lachnospiraceae_NA_.20287_ $688 . \overline{1}-$ Genome Set 771986 N/A ICE Firmicutes

13̄14_6_39_Firmicutes_Clostridia_Clostridiales_Ruminococcaceae_NA_.13414_ $639 . \overline{3}-$ Genome Set $446566-$ N/A ICE Firmicutes

UMMGS1436_ERR1190898_bin.13_Firmicutes_Clostridia_Clostridiales_Lachnospir aceae_Blāutia_NODE_249_lenḡth_74275_cov_8.936486 ${ }^{-}$Genome set 74275 N/A ICE Firmicutes

NZ_KB905466.1_Bacteroides_salyersiae_WAL_10018_=_DSM_18765_=_JCM_12988_ge

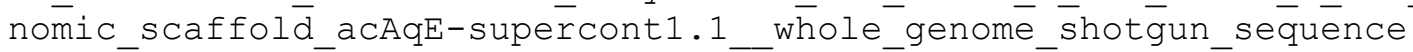
Genome set $3080894 \quad$ N/A $\bar{I} C E$ Bacteroìdetes

12718_7_72_Firmicutes_Clostridia_Clostridiales_NA_NA_.12718_7_72.3 Genome set $3372 \overline{6} 9 \quad$ N/A ICE Firmicutes

20298_3_81_Firmicutes_Clostridia_Clostridiales_Lachnospiraceae_Blautia_.2 $0298 \overline{3} \overline{8} 1 . \overline{1}$ Genome Set $605888-$ N/A ICE Firmicutes 13470 $\overline{2}$ _93_Firmicutes_Clostridia_Clostridiales_Lachnospiraceae_NA_.13470_ 2 93. ${ }^{-}$- Genome Set 256258 N/A ICE Firmicutes

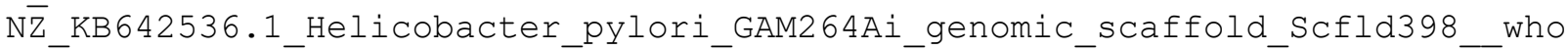
le_genome_shotgun_sequence Genome set $36 \overline{700 ~ N / A}$ N/A Próteobacteria UMGGS795_SRRR127589̄_bin.24_Firmicutes_Clostridia_Clostridiales_Ruminococca

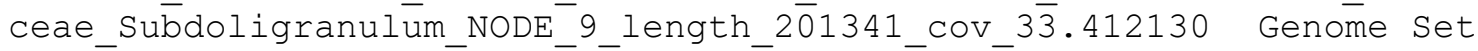
$201341 \quad \mathrm{~N} / \mathrm{A}^{-}$ICE $\overline{-}^{-}$Firmicutes

UMGS1038_ERR525948_bin.22_Tenericutes_Mollicutes_Erysipelotrichales_Erysi pelotrichaceae_Massiliomicrobiota_NODE_468_length_22553_cov_4.89444

Genome sēt 22553 N/A ICE Tenericutes

13414_6_33_Firmicutes_Clostridia_Clostridiales_Lachnospiraceae_NA_.13414_ $633 . \overline{1} 2^{-}$Genome Set 139588 N/A ICE Firmicutes

13414_6_67_Firmicutes_Clostridia_Clostridiales_Lachnospiraceae_NA_.13414_ 6_67. $\overline{3}_{-}$- Genome Set 198237 N/A ICE Firmicutes

UMMGS472_ERR1578619_bin.63_Firmicutes_Clostridia_Clostridiales_Lachnospira

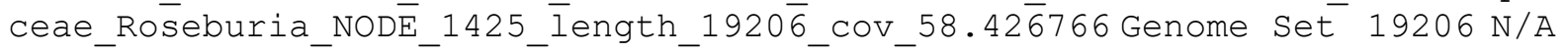
ICE Firmicutes

20298_3_64_Firmicutes_Clostridia_Clostridiales_Ruminococcaceae_NA_.20298_ 3_64.5 - Genome Set 228113 - N/A ICE Firmicutes

UMMGS800_ERR1293918_bin.21_Bacteroidetes_Bacteroidia_Bacteroidales_Porphyr omonadaceae_NA_NODE_ 37 length_103190_coV_21.209124 Genome Set $10 \overline{3} 190$

N/A ICE Bacteroidetes

NZ_JH379434.1_Prevotella_stercorea_DSM_18206_genomic_scaffold_Scfld803_W hole_genome_shotgun_sequēence Genome set $3802 \overline{6}$ N/A ICE Bactēeroidetes UMGS1735_ERR525802_bin.17_Firmicutes_Clostridia_Clostridiales_Ruminococca ceae_NA_N̄ODE_345_lēength_29̄864_cov_12.8401 Genome set $2986 \overline{4}$ N/A ICE Firmicutes

NZ_GG657553.1_Holdemania_filiformis_DSM_12042_genomic_scaffold_Scfld2_wh olē_genome_shotgun_sequence Genome set $54354 \overline{6}$ N/A ICE Tenericutes

13414_6_2_Firmicutes_Clostridia_Clostridiales_NA_NA_.13414_6_2.6 Genome set $229 \overline{4} 62 \quad$ N/A $\quad$ ICE Firmicutēes

UMGS1064_ERR321174_bin.8_Bacteroidetes_Bacteroidia_Bacteroidales_Porphyro monadaceāe_Parabactereroidēs_NODE_562_leñgth_11536_cōv_4.26017 Gēnome Set $1153 \overline{6} \mathrm{~N} / \mathrm{A} \quad$ ICE Bacteroīetes

NZ_LN877937.1_Gabonia_massiliensis_strain_GM3_whole_genome_shotgun_seque nce Genome set $2135 \overline{7} 0 \quad$ N/A $\overline{I C E}$ Bäcteroidetes 
13414_6_67_Firmicutes_Clostridia_Clostridiales_Lachnospiraceae_NA_.13414 6 67.57- Genome Set 630 N/A N/A Firmicutes

UMMGS381_ERR1190860_bin.15_Actinobacteria_Actinobacteria_Coriobacteriales_ Coriobacteriaceae $\bar{C}$ ollinsēlla NODE 46 length 87708 cov $\overline{2} 6.695709$

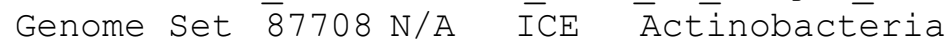

NZ_GG657592.1_Clostridium_asparagiforme_DSM_15981_genomic_scaffold_Scfld6 whole_genomé_shotgun_sequence Genome set $335 \overline{6} 774 \quad \bar{N} / \mathrm{A} \quad$ ICE

Firmicutes

13414_6_66_Firmicutes_Clostridia_Clostridiales_Lachnospiraceae_Blautia_.1 $3414 \overline{6} \overline{6} 6 . \overline{2} 3$ Genome set $413 \overline{75} \mathrm{~N} / \mathrm{A}$ ICE Firmicutes

20298_ 3 57 Actinobacteria_Actinobacteria_Coriobacteriales_Coriobacteriace ae_NA.$\overline{2} 02 \overline{9} 8$ 3 57.9 Geñome set 126302 N/A ICE Āctinobacteria UMGGS709_SRR2 $\overline{9} 9 \overline{2} 911$ bin.12_Firmicutes_Clostridia_Clostridiales_Oscillospir aceae Oscillibacte $\bar{r}$ NODE 3139 length 3595 cov $2 \overline{0} .7613$ Genome set 3595 N/A Plasmid Firmicutes

13470_2_64_Bacteroidetes_Bacteroidia_Bacteroidales_Bacteroidaceae_Bactero ides_.13470̄ 2_64.11 Gēnome set $10 \overline{1} 949$ N/A ICE Bacteroīetes NZ_KI 271072.1 _Eubacterium_ramulus_ATCC_29099_genomic_scaffold_Scaffold102 7_whole_genome_shotgun_sequence Genome Set 109213 N/A ICE Firmicutes

21673_4_22_Firmicutes_Clostridia_Clostridiales_Ruminococcaceae_Ruminococc us_.21 $6 \overline{7} 3 \overline{4} 22.3$ Genome Set $326 \overline{724}$ N/A $\overline{\text { ICE }}$ Firmicutes

NZ_JH6010990.1_Megamonas_funiformis_YIT_11815_supercont1.1_whole_genome_s hotgun sequence Genome Set 1636111 N/A ICE Firmicutes

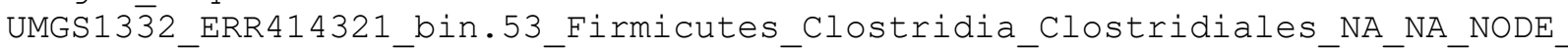

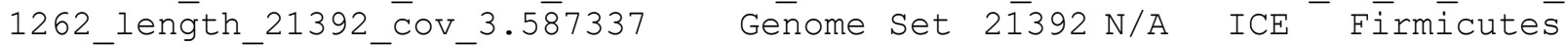
NZ A $\bar{C} F Y 01000057.1$ Roséburia inulinivorans DSM 16841 R inulinivorans1.0.1_Cont70.1_whole_genome_shotgun_sequence Genome $\overline{\text { Set }} 67763$ N/A ICE Firmicutes

UMGS1377_ERR1305905_bin.49_NA_NA_NA_NA_NA_NODE_113_length_96914_COV_14.42 1881 Geñome set $96 \overline{9} 14 \mathrm{~N} / \mathrm{A}^{-} \overline{\mathrm{ICE}}{ }^{-} \overline{\mathrm{NA}}$

14207_7_1_Firmicutes_Clostridia_Clostridiales_Lachnospiraceae_Lachnospira $.142 \overline{0} 7^{-} 7^{-} 1.8$ Genome Set $17 \overline{6373}$ N/A ICE Firmicutes

UMMGS1_SRRR5275454_bin.24_Firmicutes_Clostridia_Clostridiales_Ruminococcac eae_Anāeromassilib̄acillus _NODE_17_lēength_211925̄cov_26.8923 - Genome Set $211925 \quad$ N/A ICE Firmicutes

NZ_DS499707.1_Clostridium_scindens_ATCC_35704_Scfld_02_29_genomic_scaffol d_whole_genome_shotgun_sequence $\overline{\text { Genome }}$ Set $\overline{132727}-\overline{\mathrm{N}} / \mathrm{A}$ ICE

Firmicutes

NZ_AKCA01000001.1_Bifidobacterium_bifidum_NCIMB_41171_cont1.1_whole_geno me_shotgun_sequence Genome Set ${ }^{-} 2216395$ N/A ICE Actinobacteria UMGSS03_SRR5127411_bin.10_Firmicutes_Clostridia_Clostridiales_Peptostrept

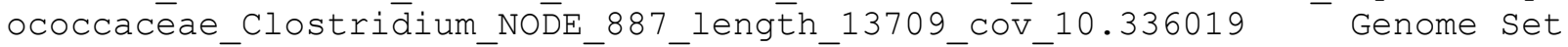
$1370 \overline{9} \mathrm{~N} / \mathrm{A} \quad \mathrm{ICE}$ Firmicutēs

UMGS 456 ERR209453 bin.40 Firmicutes Clostridia Clostridiales Ruminococcac

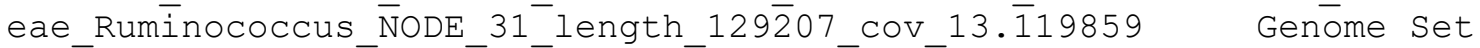
$129207 \quad$ N/A ICE Firmicutes

UMGS44_SRR5963149_bin.7_Firmicutes_Clostridia_Clostridiales_Clostridiales _Family_XI_Peptonīphilus_s_NODE_5_length_495313_Cov_32.765189 Genome Set $4 \overline{953 \overline{13}} \quad$ N/A I $\bar{C} E$ Firmicutes

NZ_GL834368.1_Clostridium_symbiosum_WAL-

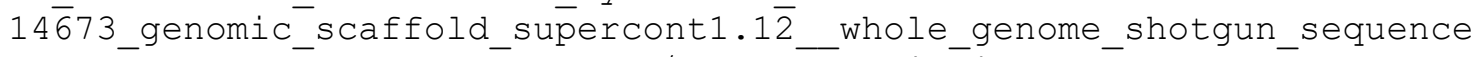
Genome set $10678 \overline{7} \quad$ N/A ICE Firmicutes 
UMGS1212 ERR525936 bin.27 Firmicutes_Clostridia_Clostridiales Ruminococca

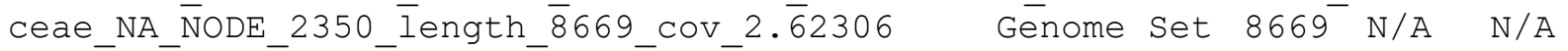
Firmicütes

UMGS1377 ERR1305905 bin.49 NA NA NA NA NA NODE 1108 length 33341 cov 14.2 42144 Geñome set $33 \overline{3} 41 \mathrm{~N} / \mathrm{A}^{-} \overline{\mathrm{ICE}}^{-} \overline{\mathrm{NA}}$

NZ_GL883855.1_Paraprevotella_xylaniphila_YIT_11841_genomic_scaffold_Scfld 99_whole_genome_shotgun_sequence Genome Set 2045 N/A ICE

Bacteroidetes

14207_7_43_Firmicutes_Clostridia_Clostridiales_Lachnospiraceae_NA_.14207_ $743 . \overline{1} 4^{-}$- Genome Set $70712 \mathrm{~N} / \mathrm{A}^{-}$ICE Firmicutes

20̄287_6_4_Proteobacteria_Betaproteobacteria_Burkholderiales_Alcaligenacea e_Achromob̄acter_.20287_6_4.4 Genome set $710 \overline{1} 99 \quad$ IncP, IncQ2 ICE Proteobacteria

UMGS1144_SRR5032340_bin.7_Firmicutes_Clostridia_Clostridiales_NA_NA_NODE_ 4_length_260214_cov_14.297806 Genome Set $2 \overline{6} 0214 \quad$ N/A ${ }^{-}$I $\bar{C} E$ Firmicutes

UMGS1963_DRR042312_bin.16_Actinobacteria_Actinobacteria_Coriobacteriales_ Coriobacteriaceae_Eggerthēlla_NODE_1191_Ēength_18493_cov_3.711804

Genome set $\overline{1} 8493 \mathrm{~N} / \mathrm{A}$ ICE $\bar{A} c t i n \overline{o b a c t e r i a}$

14207_7_23_Bacteroidetes_Bacteroidia_Bacteroidales_Bacteroidaceae_Bactero

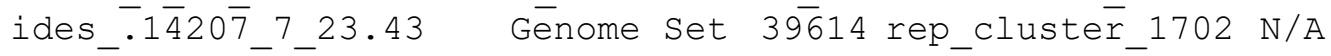

Bacteroìdetes

NZ_GG705181.1_Bacteroides_sp._2_1_22_genomic_scaffold_supercont1.9_whole genome_shotgun_sequence Genome Set $9075 \overline{5}$ N/A ICE Bacteroidetes

UMGS1711̄_SRR5558284_bin.37_Firmicutes_Clostridia_Clostridiales_Peptostrep tococcacēae_Clostrid̄ium_NODE_47_length_168564_cov_8.303519 Genome Set $16856 \overline{4} \quad$ N/A $\bar{N} / A \quad$ Firmicutes

UMGS968_ERR525795_bin.39 Firmicutes_Clostridia_Clostridiales Ruminococcac eae_NA_NODE_1183_Iength_15116_cov_4.097669 Genome Set $151 \overline{1} 6$ N/A ICE Firmicutes

UMGS622_ERR321346_bin.18_Bacteroidetes_Bacteroidia_Bacteroidales_Bacteroi daceae_Bacteroides_NoDE_T55_length_11955_cov_8.86403 Genome Set

$\overline{1} 1955 \mathrm{~N} / \mathrm{A} \quad \mathrm{IC} E \quad \bar{B}$ actēroidetés

13414_6_37_Firmicutes_Clostridia_Clostridiales_NA_NA_.13414_6_37.12 Genome set $8998 \overline{6}$ N/A ICE Firmicutes

NZ_DS996841.1_Eubacterium_biforme_DSM_3989_Scfld5_genomic_scaffold_whole _genome_shotgün_sequence_Genome set $70 \overline{8} 572$ N/A ICE

Tenericutes

NZ_ACFY01000054.1_Roseburia_inulinivorans_DSM_16841_R_inulinivorans-

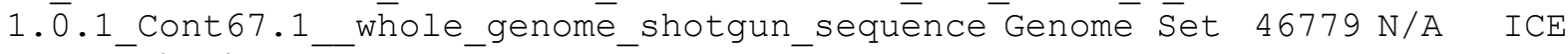
Firmicutes

NZ_LN869528.1_Intestinimonas_sp._GD2_genome_assembly_Intestinimonas_massi liēensis_scaffold_scaffold00006_whole_genome_shotgun_sequence Genome set $804651 \quad \overline{\mathrm{N}} / \mathrm{A} \quad \mathrm{ICE} \quad$ Firmicutes

UMGS961_SRR5057100_bin.26_Firmicutes_Clostridia_Clostridiales_Clostridiac

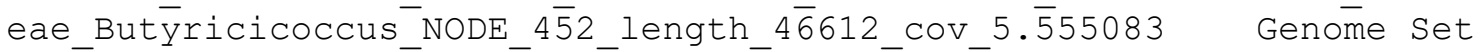
46612 N/A ICE Firmicutes

UMGS17_SRR3737013_bin.48_Firmicutes_Clostridia_Clostridiales_Peptostrepto

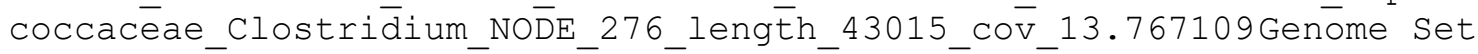
$430 \overline{15} \mathrm{~N} / \mathrm{A}$ ICE Firmicutes

UMGS218_SRR5580022_bin.2_Bacteroidetes_Bacteroidia_Bacteroidales_Bacteroi daceae_Bacteroides_NODE_133_length_36504_COV_6.291048 Genome $\overline{\text { Set }}$ $\overline{3} 6504 \mathrm{~N} / \mathrm{A} \quad \mathrm{IC} E \quad \bar{B}$ acteroidetes 
13414_6_50_Firmicutes_Clostridia_Clostridiales_Lachnospiraceae_Dorea_.134 $146 \overline{50.2}$ - Genome Set 378707 - N/A ICE Firmicutes NZ_KË150409.1_Lachnospiraceae_bacterium_3_1_57FAA_CT1_acsNUsupercont1.5_whole_genome_shötgun_sequence ${ }^{-}$Genome Set 184717 N/A ICE Firmicutes

18048 2_67 Firmicutes_Clostridia_Clostridiales_Lachnospiraceae_Blautia_.1 $8048 \overline{2} \overline{6} 7 . \overline{2}$ Genome Set 443033 - N/A ICE Firmicutes

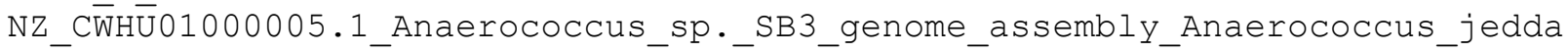
heñse_contig_contig00005_whole_e_enomé_shotgün_sequence Genome set 1226270 rep_cluster_1663 ICE Firmicutes

UMGS456_ERR209453_bin.40_Firmicutes_Clostridia_Clostridiales_Ruminococcac

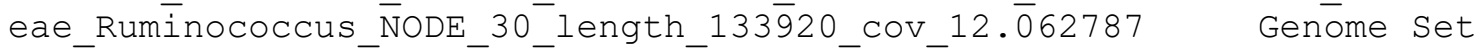
$133920 \quad$ N/A I $\overline{C E}$ Firmicutes

UMGS1140 ERR1018283 bin.51 Tenericutes Mollicutes Erysipelotrichales Erys

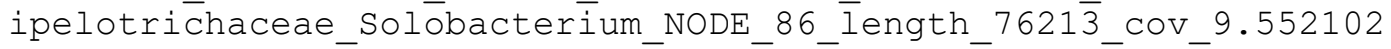

Genome Set 76213 N/A ICE Tenericutes

NZ_HE611017.1_Senegalimassilia_anaerobia_JC110_type_strain_JC110T_genom ic_scaffold_scaffold00004_whöle_genome_shotgun_sequenceGeñome set $264112 \quad$ N/A ICE Actinobacteria

UMGS179_ERR589512_bin.12 Firmicutes_Clostridia_Clostridiales Ruminococcac eae_NA_NoDE_319_lēength_65025_cov_23.740326 Genome set $650 \overline{2} 5$ N/A ICE Firmicutes

21673_4_80_Firmicutes_Clostridia_Clostridiales_Peptostreptococcaceae_Clos

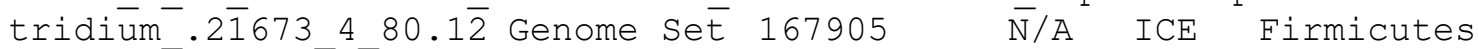
UMGS 963 SRR $430 \overline{5} 0 \overline{4} 1$ bin. 1 Bacteroidetes Bacteroidia Bacteroidales Bacteroi

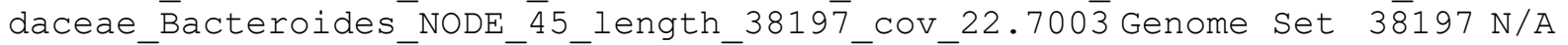

ICE Bacteroidetes

NZ_JH376420.1_Clostridium_citroniae_WAL-

171̄08_genomic_scaffold_supercont1.1_whole_genome_shotgun_sequence Genome set $21947 \overline{8} 5$ N/A ICE Firmicutes

NZ_GG705182.1_Bacteroides_sp._2_1_22_genomic_scaffold_supercont1.10_whol e_genome_shotgun_sequence Genome set $7386 \overline{9}$ rep_cluster_1702 ICE

Bacteroidetes

13414_6_32_Firmicutes_Clostridia_Clostridiales_Lachnospiraceae_Blautia_.1 $3414 \overline{6} \overline{3} 2 . \overline{1}$ Genome Set $593637-$ N/A ICE Firmicutes

UMGST1433_SRR6028647_bin.11_Bacteroidetes_Bacteroidia_Bacteroidales_Prevot

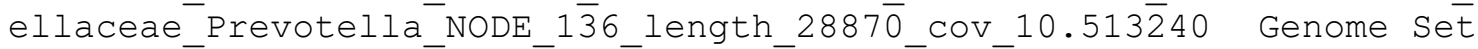
$28 \overline{8} 70 \mathrm{~N} / \mathrm{A} \quad \mathrm{ICE} \quad$ Băcteroidetes

13470_2_71_Tenericutes_Mollicutes_Erysipelotrichales_Erysipelotrichaceae_ NA_.1 $\overline{3} 4 \overline{7} 0 \overline{2}_{2} 71.3$ Genome Set $2439 \overline{95}$ N/A ICE Tenericutes

UMGGS1598_ERR $\bar{E} 414334$ bin.22_Bacteroidetes_Bacteroidia_Bacteroidales_Prevote

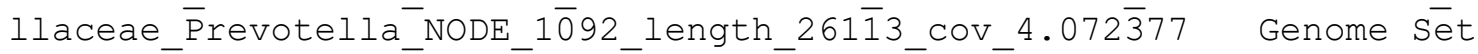

$2 \overline{6} 113 \mathrm{~N} / \mathrm{A} \quad \mathrm{N} / \mathrm{A} \quad \bar{B} \mathrm{acte} \overline{\mathrm{r}} \mathrm{i} i \mathrm{det} \overline{\mathrm{s}}$

UMGS1056_SRR3992965_bin.74_Firmicutes_Clostridia_Clostridiales_NA_NA_NODE 1785 length_19243_Cov_5.429354 Genome Set $19 \overline{2} 43$ N/A ICE Firmicutes UMMGS200_SRR6028624_bin.16_Firmicutes_Clostridia_Clostridiales_Ruminococca

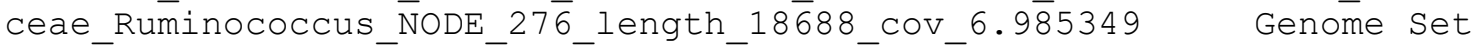
18688 N/A Plasmid - Firmicutes

UMGS1601 SRR2155375 bin.1 Firmicutes Clostridia Clostridiales Ruminococca

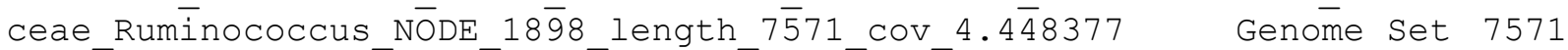
N/A ICE Firmicutes

UMGS1217_SRR1761720_bin.9_Tenericutes_NA_NA_NA_NA_NODE_44_length_92739_CO

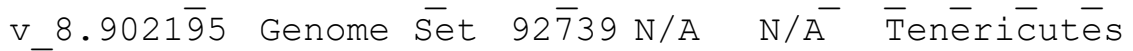


12718_7_72_Firmicutes_Clostridia_Clostridiales_NA_NA_.12718_7_72.5 Genome Set $2555 \overline{0} 3 \quad$ N/A - ICE Firmicutes

NZ_ADDR02000002.1_Desulfovibrio_sp._3_1_syn3_acqcX-

supercont2.2 whole genome shotgun sequence- Genome set 721572 N/A ICE Proteobacteria

20298_3_71_Firmicutes_Clostridia_Clostridiales_NA_NA_.20298_3_71.2 Genome set $9090 \overline{1} 1 \quad$ N/A ICE Firmicutes

NZ_JH594596.1_Odoribacter_laneus_YIT_12061_supercont1.1_whole_genome_sho tgünsequence ${ }^{-}$Genome set $2957506^{-}$N/A ICE Bacteroidetes

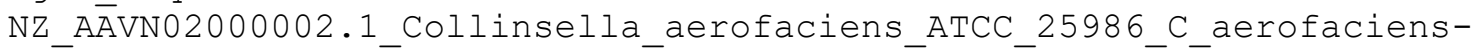

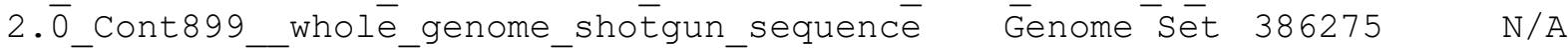
ICE Actinobacteria

UMGS1677_ERR1190831_bin.20_Firmicutes_Clostridia_Clostridiales_Peptostrep

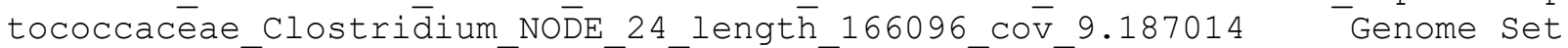
$16609 \overline{6} \quad$ N/A N/A Firmicutes

UMGS1710 ERR1293845 bin.21 Bacteroidetes Bacteroidia Bacteroidales Bacter

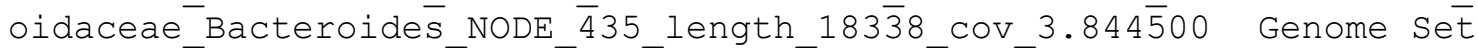
$18 \overline{3} 38 \mathrm{~N} / \mathrm{A} \quad \mathrm{ICE}^{-}$Bacterōidetes

NZ_ABJL02000003.1_Bacteroides_intestinalis_DSM_17393_B_intestinalis-

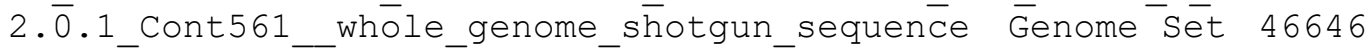

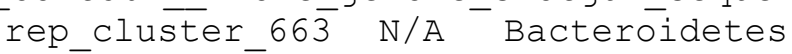

18048 2_90 Bacteroidetes_Bacteroidia_Bacteroidales_Porphyromonadaceae_odo

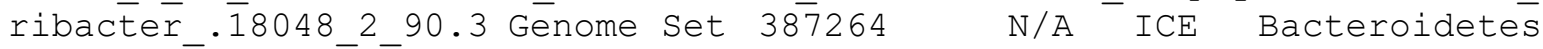
14207_7_10_Firmicutes_Clostridia_Clostridiales_Lachnospiraceae_NA_.14207_ $710 . \overline{4}-$ Genome Set 362551 N/A ICE Firmicutes

21673_4_12_Firmicutes_Clostridia_Clostridiales_Lachnospiraceae_NA_.21673_ $412 . \overline{1} 4^{-}$Genome Set $115548-$ N/A ICE Firmicutes UMMGS780_ERR1913071_bin.12 Bacteroidetes Bacteroidia Bacteroidales Prevote

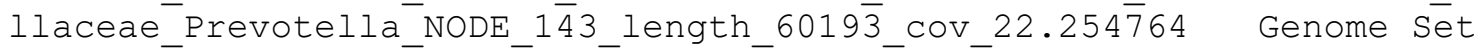
$6 \overline{0193} \mathrm{~N} / \mathrm{A} \quad \mathrm{IC} E$ Bactéroidetēs

UMGS1762_SRR1761711_bin.32_Bacteroidetes_Bacteroidia_Bacteroidales_Prevot

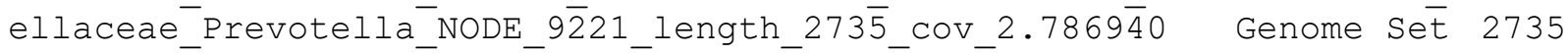
N/A N/A Bacteroìidetes

14207_7_56_Firmicutes_Clostridia_Clostridiales_NA_NA_.14207_7_56.4 Genome set $2847 \overline{6} 9 \quad$ N/A ${ }^{-}$ICE Firmicütes

UMGS1834_SRR5127700_bin.13_Actinobacteria_Actinobacteria_Coriobacteriales _Coriobacteriaceae_Collinsēlla_NODE_1210_İength_8641_Cov_5.730142

Genome set $8 \overline{6} 41$ N/A N/A Actinobacteria

UMGS373_ERR414242_bin.31_Bacteroidetes_Bacteroidia_Bacteroidales_Prevotel

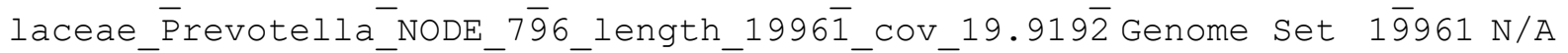
ICE Bacteroidetes

UMGS1622_ERR414356_bin.21_Firmicutes_Clostridia_Clostridiales_Lachnospira ceae_Blautia_NODE_465_length_56872_cov_9.165567 ${ }^{-}$Genome Set 56872 N/A ICE Firmicutes

NZ_JH376577.1_Paraprevotella_clara_YIT_11840_Scfld0_whole_genome_shotgun sēquence Genome set 96238 N/A $\overline{I C E}$ Bactēroidetes UMGS792_SRR5580076_bin.53_Firmicutes_Clostridia_Clostridiales_NA_NA_NODE_ 45_length_147092_cō_9.918̄831 Genome set $1 \overline{4} 7092$ N/A - I $\overline{C E}$ Firmicutes

NZ_DS995509.1_Bacteroides_eggerthii_DSM_20697_Scfld1_genomic_scaffold_wh olēegenome_shōtgun_sequence Genome Set $13348 \overline{9} 6 \quad \bar{N} / \mathrm{A}$ ICE Bacteroidetes

13470_2_93_Firmicutes_Clostridia_Clostridiales_Lachnospiraceae_NA_.13470_ 2_93.1. $7^{-}{ }^{-}$Genome Set $^{-} 72622$ N/A ${ }^{-}$ICE Firmicütes 
UMGS904_SRR1761720_bin.5_Firmicutes_Clostridia_Clostridiales_Lachnospirac eae_Lachnobacterium_NoDE_69_length_72416_cov_9.436133 Genome Set

$72416 \mathrm{~N} / \mathrm{A} \quad \mathrm{IC} \overline{\mathrm{E}} \quad \mathrm{F} \overline{\mathrm{i}} \mathrm{rm} \overline{\mathrm{i}} \mathrm{cutes}$

UMGS698 ERR414252 bin.16 Bacteroidetes Bacteroidia Bacteroidales Bacteroi daceae_Bacteroides_NODE_78_length_1170222_cov_32.496636 Genome Set
$\overline{1} 17022$
$\mathrm{N} / \mathrm{A} \quad \overline{\mathrm{I}} \mathrm{CE}-$ Bacteroidetes

13414_6_44_Firmicutes_Clostridia_Clostridiales_Lachnospiraceae_Lachnobact erium.$\overline{1} 34 \overline{1} 4644.49$ Genome SeẼ 9273 N/A ICE Firmicutes

NZ_HE $978639 . \overline{1}$ Àeromicrobium_sp._JC14_genomic_scaffold_scaffold00004_who le_genome_shotgun_sequence Geñome Set $7714 \overline{8} \mathrm{~N} / \mathrm{A}$ N/A Actinobacteria UMGGS1573_ËRR321203_bin.19_Tenericutes_NA_NA_NA_NA_NODE_1639_length_5299_C ov $3.417 \overline{6} 20$ Genome Set $52 \overline{9} 9$ N/A ICE $\bar{T}$ Tenericutēs

14207_7_95_Firmicutes_Clostridia_Clostridiales_Ruminococcaceae_NA_.14207_ 7 95. ${ }^{-}$- Genome Set 457756 N/A ICE Firmicutes

14̄207_6_9_Bacteroidetes_Bacteroidia_Bacteroidales_Bacteroidaceae_Bacteroi des_. $\overline{1} 4 \overline{2} 0 \overline{7} \_6$ 9.10Genome Set $123469^{-}$N/A ICE ${ }^{-}$Bacteroidetes

$142 \overline{0} 7770^{-}$Băcteroidetes Bacteroidia Bacteroidales Rikenellaceae Alistipe

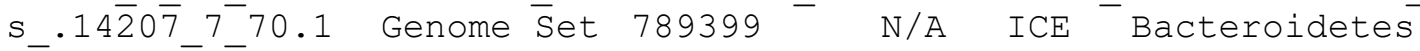

UMGS920_SRR6028474_bin.9_Firmicutes_Clostridia_Clostridiales_Lachnospirac

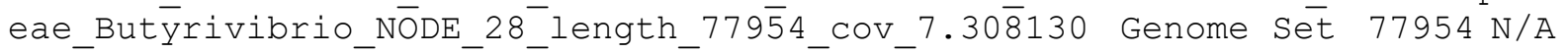

ICE Firmicutes

13414_6_44_Firmicutes_Clostridia_Clostridiales_Lachnospiraceae_Lachnobact erium_.13414_6_44.41-Genome Set 12829 N/A ICE Firmicutes

21673_4_71_Firmicutes_Clostridia_Clostridiales_Peptostreptococcaceae_Clos

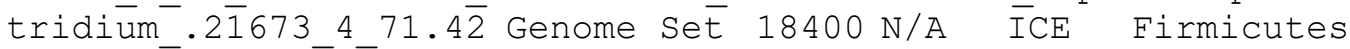

20298_3-66_Firmicutes_Clostridia_Clostridiales_Clostridiales_Family_XI_Pe

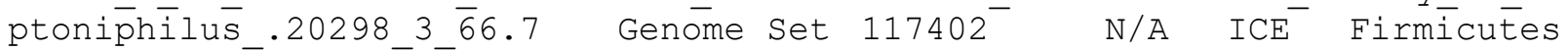
UMGS199_SRR 408188 bin.8 Firmicutes Clostridia Clostridiales Lachnospirac

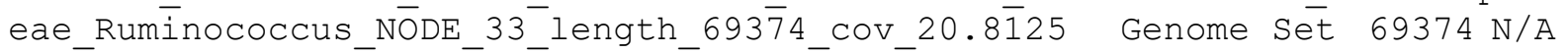

ICE Firmicutes

UMGS624_ERR011095_bin.1_Bacteroidetes_Bacteroidia_Bacteroidales_Bacteroid aceae_Bacteroides_NODE_26_length_69394_Cov_8.11064 Genome set $\overline{69394}$ N/A ICE Bacteroidetes

14207_7_56_Firmicutes_Clostridia_Clostridiales_NA_NA_.14207_7_56.15 Genome set $1091 \overline{0} 6 \quad$ N/A ${ }^{-}$ICE Firmicütes

UMGS1226_SRR5057015_bin.98_Firmicutes_Clostridia_Clostridiales_NA_NA_NODE 242_lenḡth_88696_Cov_7.526̄754 Genome set $88 \overline{696}$ N/A ICE Firmicutes 20298_3_64_Firmicutes_Clostridia_Clostridiales_Ruminococcaceae_NA_.20298_ 364.1. ${ }^{-}$Genome Set $64331 \mathrm{~N} / \mathrm{A}^{-}$ICE Firmicutes

$1 \overline{2} 718$ 7_29 Bacteroidetes_Bacteroidia_Bacteroidales_Bacteroidaceae_Bactero

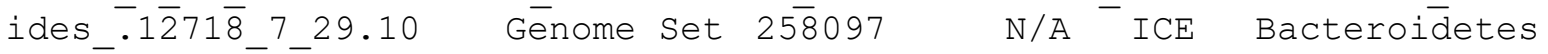
UMGS 2007 ERR $\overline{2} \overline{0} 9576$ bin.9_Bacteroidetes_Bacteroidia_Bacteroidales_Bacteroi

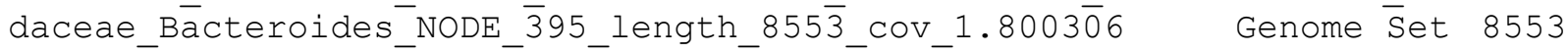

$\overline{\mathrm{N}} / \mathrm{A} \quad$ ICE Bacteroidetes

UMGS802_ERR1305895_bin.91_Firmicutes_Clostridia_Clostridiales_Ruminococca

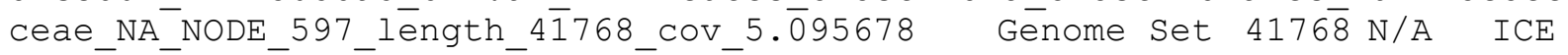

Firmicutes

NZ_JH376598.1_Paraprevotella_clara_YIT_11840_Scfld35_whole_genome_shotgu n_sequence Genome set 76759 N/A $\overline{\text { ICE }}$ Bactēeroidetes UMMGS1511_ERR1018258_bin.18_Bacteroidetes_Bacteroidia_Bacteroidales_Porphy

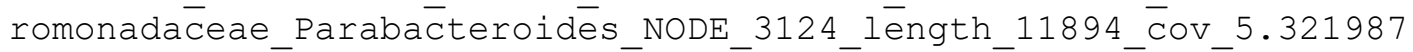

Genome Set 11894 N/A ICE Bacteroidetes 
UMGS1083_SRR2912778_bin.6_Actinobacteria_Actinobacteria_Coriobacteriales_ Coriobacteriaceae_Cōllinsēella_NODE_982_lēength_10929_cov_13.004046

Genome set $\overline{1} 0929$ N/A N/A Āctinobacteria

NZ GL538349.1 Faecalibacterium cf. prausnitzii KLE1255 genomic scaffold S cfīd712_wholèegenome_shotgun_sequence Genome- Set $26 \overline{688}$ N/A N/A

Firmicutes

20298_3_65_Firmicutes_Clostridia_Clostridiales_Lachnospiraceae_NA_.20298_ $365 . \overline{2} 6^{-}$Genome Set $38789 \mathrm{~N} / \mathrm{A}^{-}$ICE Firmicutes

UMMGS82_ERR525898_bin.19_Firmicutes_Clostridia_Clostridiales_Peptostreptoc

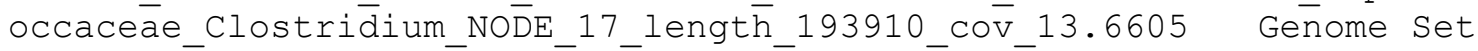
$19 \overline{3} 910$
N/A $\quad$ ICE $\bar{E}$ Firmicutes

13414_6_60_Firmicutes_Clostridia_Clostridiales_NA_NA_.13414_6_60.15 Genome set $5496 \overline{4}$ N/A ICE Firmicutes

13470264 Bacteroidetes Bacteroidia Bacteroidales Bacteroidaceae Bactero

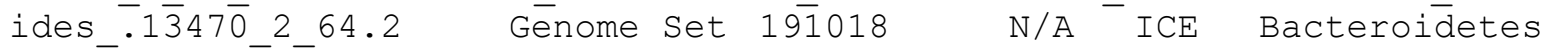
NZ_GG657591.1_Clostridium_asparagiforme_DSM_15981_genomic_scaffold_SCfld5 whole_genomé_shotgun_sequence Genome set $115 \overline{3} 127 \quad \overline{\mathrm{N}} / \mathrm{A} \quad \mathrm{ICE}^{-}$ Firmicutes

20298_3_36_Firmicutes_Clostridia_Clostridiales_Clostridiales_Family_XI_Pe

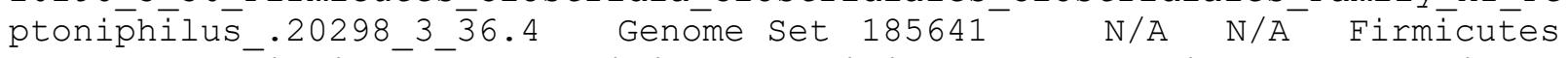
18048_1_72_Eirmicutes_clostridia_Clostridiales_Lachnospiraceae_Blautia_.1 $8048 \overline{1} \overline{7} 2 . \overline{7}$ Genome Set $199243-$ N/A ICE Firmicutes 13470_ㄷ﹎.92_Firmicutes_Clostridia_Clostridiales_Lachnospiraceae_NA_.13470_ 2 292. ${ }^{2}-$ Genome Set 211300 N/A ICE Firmicutes

NZ̄_KE150238.1_Bilophila_wadsworthia_3_1_6_genomic_scaffold_acCls-

supercont2.1_whole_genome_shotgun_sequence Genome set $3 \overline{1} 69074$ N/A

ICE Proteobacteria

21673_4_12_Firmicutes_Clostridia_Clostridiales_Lachnospiraceae_NA_.21673_ $412 . \overline{2}{ }^{-}-$Genome Set $345794-$ N/A ICE Firmicutes

UMMGS1356_ERR505088_bin.30_Firmicutes_Clostridia_Clostridiales_Peptostrept

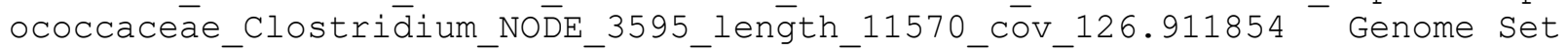
$1157 \overline{0} \mathrm{~N} / \mathrm{A}$ ICE Firmicutes

NZ_DS483486.1_Faecalibacterium_prausnitzii_M21_2_Scfld_02_7_genomic_scaff $\circ \bar{d}$ _whole_geñome_shotgun_sequence Genome Set $\overline{13} \overline{4} 67 \mathrm{~N} / \bar{A} \overline{\mathrm{N}} / \overline{\mathrm{A}}$ Firmicutes

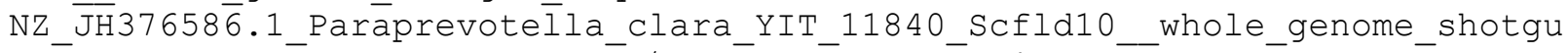
n_sequence Geñome set $60713 \overline{\mathrm{N}} / \mathrm{A}$ ICE Bactēeroidetes

NZ_KI391948.1_Ruminococcaceae_bacterium_D16_genomic_scaffold_acsPysupercont2.2_whole_genome_shōtgun_sequence Genome Set $992 \overline{6} 87$ N/A ICE Firmicutes

14207_6_13_Bacteroidetes_Bacteroidia_Bacteroidales_Bacteroidaceae_Bactero

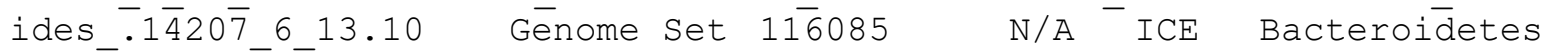

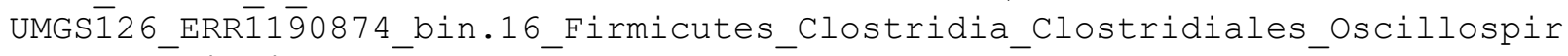
aceae_Oscillibacter_NODE_1059_length_29136_cov_ī12.198962 Genome Set

$29136 \mathrm{~N} / \mathrm{A} \quad \mathrm{ICE} \quad$ Firmicūtes

14207_6_9_Bacteroidetes_Bacteroidia_Bacteroidales_Bacteroidaceae_Bacteroi des_. $\overline{1} 4 \overline{2} 0 \overline{7} \_6$ 9.18Genome Set 76947 N/A ICE Bacteroidetes

20287_6_88_Firmicutes_Clostridia_Clostridiales_Lachnospiraceae_NA_.20287_ 6_88. $\overline{6}$ - Genome Set 304030 - N/A ICE Firmicutes

UMMGS594_SRR5963240_bin.13_Firmicutes_Clostridia_Clostridiales_Clostridial

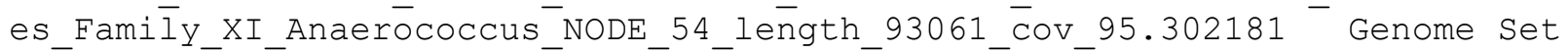
$930 \overline{61} \overline{\mathrm{N}} / \mathrm{A} \quad$ ICE Firmicutes

UMGS881_SRR4033077_bin.13_Firmicutes_Clostridia_Clostridiales_Lachnospira ceae_Psēudobutyrivibrio_NŌDE_17_length_71722_COV̄_19.268785 - Genome Set 71722 N/A ICE Firmicutēes 
UMGS126_ERR1190874_bin.16_Firmicutes_Clostridia_Clostridiales_Oscillospir

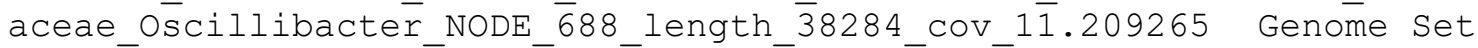

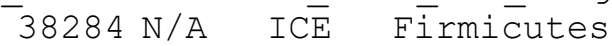

20298363 Bacteroidetes Bacteroidia Bacteroidales Rikenellaceae Alistipe

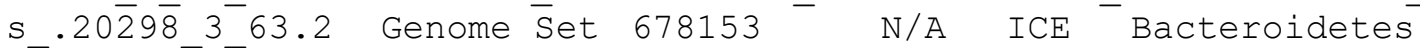

UMGS1759_ERR209240_bin.10_Firmicutes_Clostridia_Clostridiales_Lachnospira

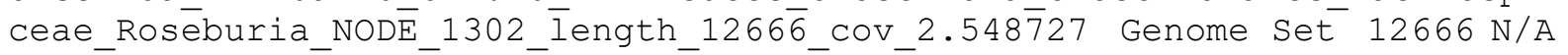

ICE Firmicutes

14207_7_56_Firmicutes_Clostridia_Clostridiales_NA_NA_.14207_7_56.26

Genome Set $3075 \overline{7}$ N/A N/A Firmicutes

NZ_CCYK01000002.1_Pseudomonas_sp._CB1_genome_assembly_Pseudomonas_massili ens̄is_CB1_contig_contig00002_whōle_genome_shotgun_sēquence Geñome set $139 \overline{806} \quad \overline{\mathrm{N}} / \mathrm{A} \quad \mathrm{N} / \mathrm{A} \quad \overline{\text { Proteobacteria }}$

NZ KE993597.1 Clostridium sp. ATCC 29733 genomic scaffold Scaffold30 who le_genomesshōtgun sequence Genome set $\overline{5} 3479 \mathrm{~N} / \overline{\mathrm{A}}$ ICE $\overline{\text { Firmicutes }}$

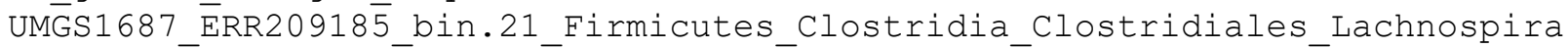

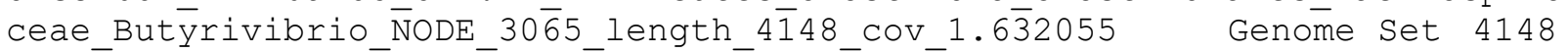
N/A N/A Firmicutes

UMGS664_ERR209886_bin.11_Firmicutes_Clostridia_Clostridiales_Ruminococcac

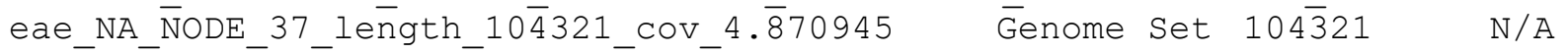

ICE Firmicutes

UMGS695_ERR1913046_bin.9_Tenericutes_Mollicutes_Erysipelotrichales_Erysip elotrichaceae_Solob̄acterium_NODE_903_length_1693̄1_cov_5.409635 Genome Set $16931 \mathrm{~N} / \overline{\mathrm{A}}$ Plasmid Tenericütes

18048_1_69_Firmicutes_Clostridia_Clostridiales_Lachnospiraceae_NA_.18048_ $169 . \overline{7}-$ Genome Set 145375 - N/A ICE Firmicutes

NZ_LN898230.1_Tissierellia_bacterium_GD9_genome_assembly_Micromassilia_ti moñensis_scaffold_scaffold̄00008_whole_genome_shotgun_sequence Genome Set 1049559 N/A ICE Firmicutes

NZ_GG662014.1_Coprococcus_comes_ATCC_27758_genomic_scaffold_Scfld9_whole _gēnome_shotgün_sequence - Geñome Set $89 \overline{1565}{ }_{\text {N }}$-A ICE Firmicutes 20298_3_35_Firmicutes_Clostridia_Clostridiales_Lachnospiraceae_Blautia_.2 $0298 \overline{3} \overline{3} 5 . \overline{6}$ Genome Set 158206 - N/A ICE Firmicutes

UMGS $\overline{6} 3 \overline{6}$ _ERR1305895_bin.68_Tenericutes_Mollicutes_NA_NA_NA_NODE_868_length 33424 COV_12.07608̄9 Geñome Set $334 \overline{2} 4$ N/A N/A Tenericutes

UMGS595_SRR̄5127461_bin.3_Actinobacteria_Actinobacteria_Coriobacteriales_C oriobacteriaceae_Cöllinsēlla_NODE_2_lenḡth_139026_cov_- 8.832699 Genome sēt 139026

N/A ICE Actinobacteria

NZ_JH414700.1_Subdoligranulum_sp._4_3_54A2FAA_genomic_scaffold_supercont1 .3_whole_genome_shotgun_sequence Genome set 325285 - N/A ICE Firmicutes

UMGS1054_SRR5056649_bin.8_Firmicutes_Clostridia_Clostridiales_NA_NA_NODE_ 7693 lenğth_5966_cov_10.379124 Genome Set $5 \overline{9} 66$ N/A ICE F UMGST̄073_ERR $1190 \overline{9} 46$ bin.14_Bacteroidetes_Bacteroidia_Bacteroidales_Prevot

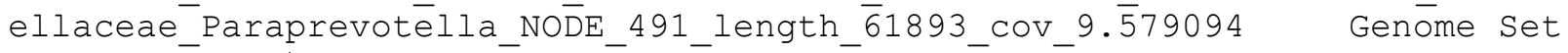

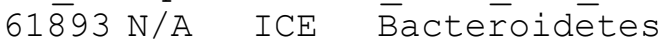

11861_6_65_Firmicutes_Clostridia_Clostridiales_Ruminococcaceae_NA_.11861_ 6_65.10- Genome Set 163406 N/A ICE Firmicutes

200298_3_8_Tenericutes_Mollicutes_Erysipelotrichales_Erysipelotrichaceae_N A_.20 $\overline{2} 9 \overline{8}$ 3 8.4 Genome Set $2182 \overline{18}$ N/A ICE Tenericutes UMGGS462_ERRT1305881_bin.83_Firmicutes_Clostridia_Clostridiales_NA_NA_NODE_ 27_length_226082_cov_9.547497 Genome Set $2 \overline{2} 6082$ N/A I $\overline{C E}$

Firmicutes 
UMGS285_SRR1201570_bin.8_Firmicutes_Clostridia_Clostridiales_NA_NA_NODE_7 0_length̄63969_cov_10.3672 Genome Set $63969 \overline{\mathrm{N}} / \mathrm{A}$ ICE Firmicutes UMMGS1869 ERR414339-bin.30 Bacteroidetes Bacteroidia Bacteroidales Bactero idaceae Bacteroides NODE $\overline{3} 17$ length $580 \overline{3} 7 \mathrm{cov} 6.232 \overline{3} 31$ Genome Set $5 \overline{8} 037 \mathrm{~N} / \mathrm{A} \quad \mathrm{IC} \overline{\mathrm{E}}$ Băcteroidetes 20287653 Bacteroidetes_Bacteroidia_Bacteroidales_Bacteroidaceae Bactero ides $.2 \overline{0} 28 \overline{7} 653.10$ Genome set 296783 N/A ICE Bacteroīetes UMGST2027_SRR̄1761721_bin.40_Bacteroidetes_Bacteroidia_Bacteroidales_Prevot

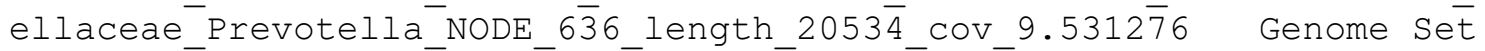
$20 \overline{5} 34 \mathrm{~N} / \mathrm{A} \quad \mathrm{IC} \overline{\mathrm{E}}$ Bācteroidetes

NZ_LT594610.1_Butyricimonas_sp._Marseille-

P2 440 _whole_ḡenome_shotgun_sequence Genome set 2198421 N/A ICE Bacteroidetes

14207613 Bacteroidetes Bacteroidia Bacteroidales Bacteroidaceae Bactero ides_.14 $20 \overline{7} \_6 \_13.27$ Genome set $59 \overline{5} 53$ N/A ICE Bacteroidetes NZ_DS547021.1_Clostridium_sp._SS2_1_Scfld_03_25_genomic_scaffold_whole_g enōme shotgun sequence Geñome Set $1 \overline{4} 1704^{-}{ }^{-}$N/A ICE ${ }^{-}$Firmicutes UMGS536_SRR5275432_bin.34_Firmicutes_Clostridia_Clostridiales_Ruminococca

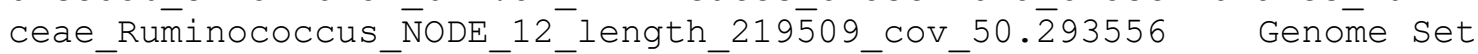
$219509 \quad \overline{\mathrm{N}} / \mathrm{A} \quad$ ICE Firmicutes

UMGS1869_ERR414339_bin.30_Bacteroidetes_Bacteroidia_Bacteroidales_Bactero

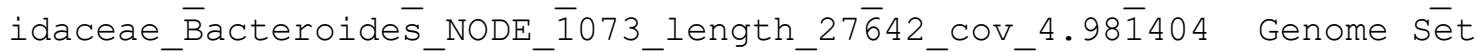
$27642 \mathrm{~N} / \mathrm{A} \quad \mathrm{ICE} \quad$ Băcteröidetes

UMGS1630_SRR453564_bin.8_Firmicutes_Clostridia_Clostridiales_Peptostrepto

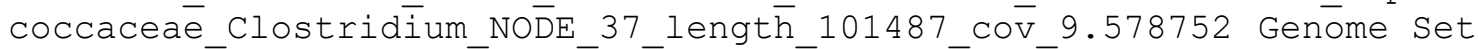
$101 \overline{4} 87$
$\mathrm{N} / \mathrm{A}-\mathrm{ICE}^{-}$Firmicutes

20287_6_4_Proteobacteria_Betaproteobacteria_Burkholderiales_Alcaligenacea e_Achromob̄acter_.20287_6_4.7 Genome set $400 \overline{1} 92$ rep_cluster_10 ICE

Proteobacteria

13414_6_17_Firmicutes_Clostridia_Clostridiales_Lachnospiraceae_Ruminococc us_. $1 \overline{3} 4 \overline{1} 4 \bar{\sigma}_{-} 17.18$ Genome Set $617 \overline{45} \mathrm{~N} / \mathrm{A}$ ICE Firmicutes

13470_2_93 $\overline{3}$ Firmicutes_Clostridia_Clostridiales_Lachnospiraceae_NA_.13470_ 2 293.5 $2^{-}$- Genome Set 2402 N/A ${ }^{-}$Plasmid Firmicutes

NZ_JH376872.1_Clostridium_clostridioforme_2_1_49FAA_genomic_scaffold_supe rcont1.3_whole_genome_shötgun_sequence Gēomōe set 722831 - N/A ICE Firmicutes

20298_3_65_Firmicutes_Clostridia_Clostridiales_Lachnospiraceae_NA_.20298_ 3_65. $\overline{8}^{-}-$Genome Set $193131-$ N/A ICE Firmicutes

UM̄GS12_SRR5106488_bin.11_Proteobacteria_Betaproteobacteria_Burkholderiale

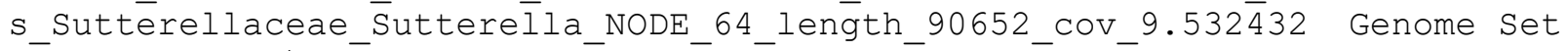
$90652 \mathrm{~N} / \mathrm{A}$ ICE Protēobacterēia

20298_3_48_Firmicutes_Clostridia_Clostridiales_Lachnospiraceae_Blautia_.2 $0298 \overline{3} \overline{4} 8 . \overline{1} 1 \quad$ Genome set $229 \overline{996} \quad$ N/A ICE Firmicutes

UMGST̄436_ERR1190898_bin.13_Firmicutes_Clostridia_Clostridiales_Lachnospir aceae_Bläutia_NODE_$\overline{7} 18$ _lenğth_35044_Cov_12.739690 Genome set $\overline{0} 35044$ N/A ICE Firmicutes

20287_6_37_Firmicutes_Clostridia_Clostridiales_NA_NA_.20287_6_37.5 Genome set $1507 \overline{3} 4 \quad$ N/A - ICE Firmicutes

UMGS26_ERR1953518_bin.80_Actinobacteria_Actinobacteria_Coriobacteriales_C oriobaçteriaceae_Ēggerthēlla_NODE_30_leñgth_230818_cov_5.550955 Genome Set 230818 N/A ICE Actinobactēria

13414_6_2_Firmicutes_Clostridia_Clostridiales_NA_NA_.13414_6_2.3 Genome set $398 \overline{1} 65$ N/Ā ICE Firmicutés 
UMGS83_ERR911953_bin.80_Firmicutes_Clostridia_Clostridiales_NA_NA_NODE_7_ length_307653_cov_12.780489 Genome set $30765 \overline{3} \quad \mathrm{~N} / \mathrm{A}$ ICE E Firmicutes $20298 \overline{3} 4$ Proteobācteria Gammaproteobacteria Enterobacteriales Enterobact

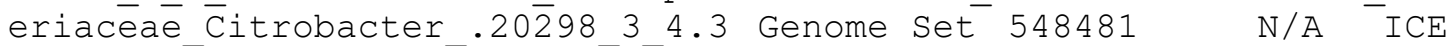

Proteobacteria

UMGS1117_SRR5127624_bin.20_Actinobacteria_Actinobacteria_Coriobacteriales _Coriobacteriaceae_Collinsēella_NODE_236_lēength_38547_cov_7.410553

Genome set $3 \overline{8} 547 \mathrm{~N} / \mathrm{A}$ ICE ACtinōbacteria

12718_7_84_Firmicutes_Clostridia_Clostridiales_Ruminococcaceae_NA_.12718_

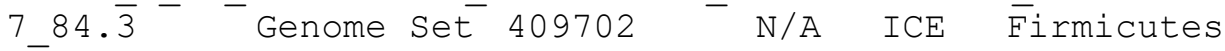

21673_4_3_Firmicutes_Clostridia_Clostridiales_Lachnospiraceae_NA_.21673_4

3.9 Genome set $121 \overline{9} 09$ N/Ā ICE Firmicutes

18048_2_82_Firmicutes_Clostridia_Clostridiales_NA_NA_.18048_2_82.7

Genome Set $9473 \overline{6}$ N/A ICE ${ }^{-}$Firmicutes

18048 2_67_Firmicutes_Clostridia_Clostridiales_Lachnospiraceae_Blautia_.1 $8048 \overline{2} \overline{6} 7 . \overline{5}$ Genome Set 317204 N/A ICE Firmicutes

1341 $\overline{4}$ 6_2_Firmicutes_Clostridia_Clostridiales_NA_NA_.13414_6_2.9

Génome set $125 \overline{0} 26 \quad$ N/A $\quad$ ICE Firmicutēs

20298_3_64_Firmicutes_Clostridia_Clostridiales_Ruminococcaceae_NA_.20298_

3_64. ${ }^{-}-$Genome Set 367086 - N/A ICE Firmicutes

18̄048_2_85_Firmicutes_Clostridia_Clostridiales_Peptostreptococcaceae_Clos tridium_.18048_2_85.7 Genome Set 136556 N/A ICE Firmicutes

UMGS177 $\overline{8}$ ERR11 $\overline{9} 0 \overline{9} 62$ bin.65_Firmicutes_Clostridia_Clostridiales_RuminococC

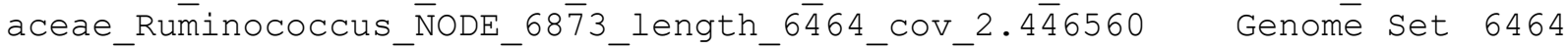

N/A ICE Firmicutes

NZ_DS995508.1_Bacteroides_eggerthii_DSM_20697_Scfld0_genomic_scaffold_wh olē_genome_shōtgun_sequence Genome $\overline{\text { Set }}{ }^{-66039 \overline{4}}$ N/A ICE

Bacteroidetes

14207_7_32_Firmicutes_Clostridia_Clostridiales_NA_NA_.14207_7_32.1

Genome set $1730 \overline{8} 4 \quad$ N/A ICE Firmicutes

20298_3_66_Firmicutes_Clostridia_Clostridiales_Clostridiales_Family_XI_Pe ptoniphīius_.20298_3_66.6 Genome Set 117522 N/A ICE Firmicutes 18048_1_72_Eirmicutes_Clostridia_Clostridiales_Lachnospiraceae_Blautia_.1 $8048 \overline{1}_{\overline{1}} \overline{7} 2 . \overline{3}$ Genome Set 400443 N/A ICE Firmicutes

$1347 \overline{0} \overline{2} 62$ Bacteroidetes Bacteroidia Bacteroidales Bacteroidaceae Bactero ides_.13470_2_62.9 Gēnome set 110983 N/A ICE Bacteroidetes

21673_4_77_Firmicutes_Clostridia_Clostridiales_Peptostreptococcaceae_Clos tridium-.21673 4 77.4- Genome Set 184413 N/A ICE Firmicutes NZ_LN913̄001.1_Ruminococcus_sp._AT10_genome_assembly_Ruminococcus_phoceens is_scaffold_scaffold00005_whöle_genome_shotgun_sequence Genome set
$216494 \overline{8}$
$\mathrm{N} / \mathrm{A} \quad \mathrm{ICE}$
Firmicutes

NZ_KI392031.1_Oxalobacter_formigenes_HOxBLS_genomic_scaffold_supercont2.2 whole_genomé_shotgun_sequence Geñome Set $18062 \overline{0} 3$ N/A ${ }^{-}$ICE

Proteobacteria

NZ_JH376605.1_Paraprevotella_clara_YIT_11840_Scfld59_whole_genome_shotgu n_sequence Genome Set $17704 \overline{1} \quad \bar{N} / A-{ }^{-}$ICE - Bacteroidetes

UMGGS19_ERR321453_bin.7_Bacteroidetes_Bacteroidia_Bacteroidales_Bacteroid aceae_Bäcteroides_NODE_ż_length_68502_Cov_2.63624 Genome Set $\overline{68502}$ N/A

ICE Bacteroidetes

20287_6_22_Firmicutes_Clostridia_Clostridiales_Ruminococcaceae_Ruminococc us_.202 $\overline{8} 7 \overline{6}_{6} 22.8$ Genome Set $875 \overline{45}$ N/A N/A Firmicutes

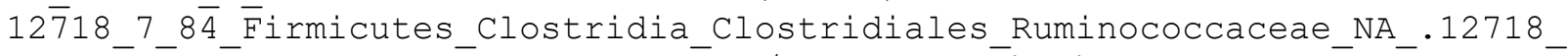

7_84. $\overline{8}-$ Genome Set $147599-$ N/A ICE Firmicutes 
NZ_CVPE01000006.1_Bacillaceae_bacterium_mt6_genome_assembly_Rubeoparvulum _mássiliensis_coñtig_contig00̄006_wholēe_geñome_shōtgun_sequence

Genome set $1233 \overline{1} 24 \quad$ N/A ICE Firmicutes

NZ DS995511.1 Bacteroides eggerthii DSM 20697 Scfld3 genomic scaffold wh olē_genome_shötgun_sequence Genome set $98985 \overline{8} \quad$ N/A ICE

Bacteroidetes

8080_1_78_Firmicutes_Clostridia_Clostridiales_Peptostreptococcaceae_Clost

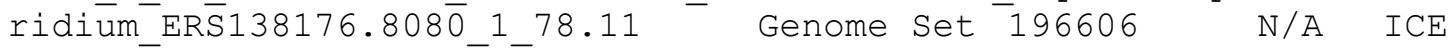

Firmicutes

8080_1_80_Tenericutes_Mollicutes_Erysipelotrichales_Erysipelotrichaceae_S olobācterium_ERS138178.8080_1_80.9Genome Set $12698 \overline{4}$ N/A ICE

Tenericutes

NZ_JH992940.1_Bacteroides_oleiciplenus_YIT_12058_genomic_scaffold_superco ntİ.1_whole_genome_shotgün_sequence - Geñome sēt $19470 \overline{8} 3$ N/Ā ICE Bacteroidetes

UMGS2010 ERR209156 bin.11 Firmicutes Clostridia Clostridiales Lachnospira

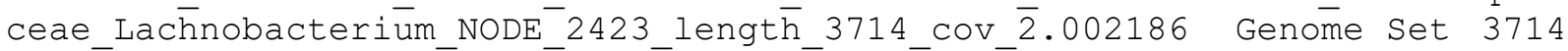
N/A N/A Firmicutes

NZ_GL834359.1_Clostridium_symbiosum_WAL-

14 $\overline{6} 73$ _genomic_scaffold_supercont1.3_whole_genome_shotgun_sequence Genome set $64796 \overline{5}$ N/A ICE Firmicutes

UMGS1699_SRR341590_bin.30_Firmicutes_Clostridia_Clostridiales_Peptostrept

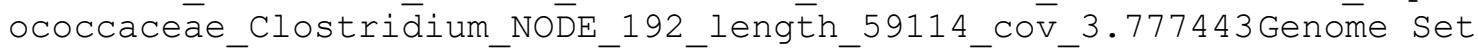
$5911 \overline{4} \mathrm{~N} / \mathrm{A} \quad \mathrm{N} / \mathrm{A} \quad$ Firmicutés

14207_7_86_Firmicutes_Clostridia_Clostridiales_NA_NA_.14207_7_86.3 Genome set $4686 \overline{1} 7 \quad$ N/A ICE Firmicutes

NZ_GG657557.1_Holdemania_filiformis_DSM_12042_genomic_scaffold_Scfld6_wh olēegenome_shötgun_sequeñce Genome $\overline{\text { Set }}{ }^{-} 41188 \overline{8} \quad \mathrm{~N} / \overline{\mathrm{A}}$ ICE

Tenericutes

11861_6_58_Firmicutes_Clostridia_Clostridiales_Lachnospiraceae_Blautia_.1 $1861 \overline{6} \overline{5} 8 . \overline{4}$ Genome Set $264389-$ N/A ICE Firmicutes

NZ_JḦ376873.1_Clostridium_clostridioforme_2_1_49FAA_genomic_scaffold_supe rcōnt1.4_whole_genome_shōtgun_sequence Gēenomē set $366225^{-}$N/A ICE Firmicutes

NZ_GG704769.1_Subdoligranulum_variabile_DSM_15176_genomic_scaffold_Scfld0

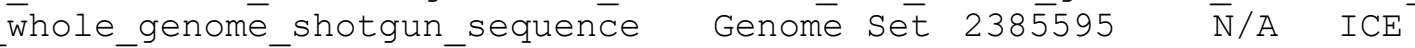
Firmicutes

NZ_JH594448.1_Eubacterium_sp._3_1_31_genomic_scaffold_supercont1.1_whole _genome_shotgun_sequence Genome Set 2298056 N/A ICE

Tenericutes

NZ_KI271191.1_Eubacterium_ramulus_ATCC_29099_genomic_scaffold_Scaffold899 whole_genoméeshotgun_sequence Genome Set 46574 N/A ICE Firmicutes $\overline{U M} G S 157 \overline{7}$ ERR1305905_biñ.1_Firmicutes_Clostridia_Clostridiales_NA_NA_NODE_

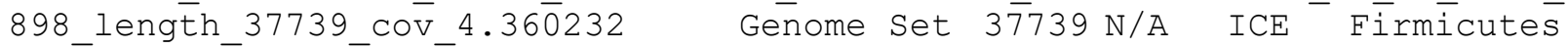

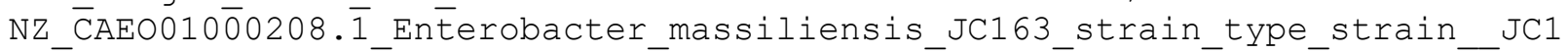
63_whole_genome_shotgun_sequeñce Genome Set $318^{-}$N/A ${ }^{-}$Plasmid

Proteobacteria

UMGS1099_ERR526047_bin.23 Firmicutes_Clostridia_Clostridiales_NA_NA_NODE_ 4608 lenḡth $6813 \mathrm{Cov} 9.42 \overline{7} 937$ Geñome set $6 \overline{813}$ N/A ICE Fírmícutes UMGST 1624 ERR $6886 \overline{4} 4$ bin. 6 Bacteroidetes Bacteroidia Bacteroidales_Prevotel

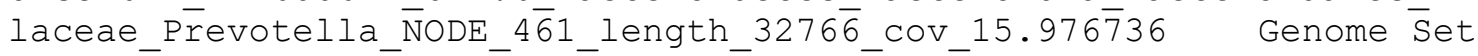

$\overline{3} 2766 \mathrm{~N} / \mathrm{A} \overline{\mathrm{I}} \mathrm{CE}$ Bacteroidēes 
UMGS1208_ERR589513_bin.7_Bacteroidetes_Bacteroidia_Bacteroidales_Prevotel

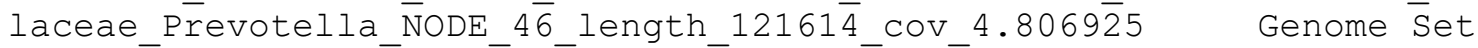
121614
$\overline{\mathrm{N}} / \mathrm{A} \quad$ ICE $\overline{\mathrm{E}}$ Bacteroidetes

UMGS1855 ERR1190896 bin.57 Bacteroidetes Bacteroidia Bacteroidales Porphy romonadaceae_NA_NODE_29_length_179755_cov_4.834841 Genome Set 179755

N/A ICE Bacteroidetes

UMGS1938_SRR5963258_bin.3 Bacteroidetes Bacteroidia_Bacteroidales_Porphyr

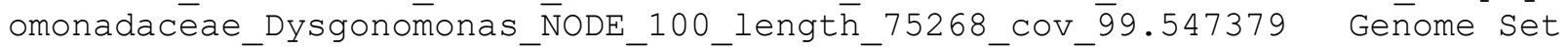

$75268 \overline{\mathrm{N}} / \mathrm{A}$ ICE Bācterōidē̄es

NZ_ACFY01000048.1_Roseburia_inulinivorans_DSM_16841_R_inulinivorans-

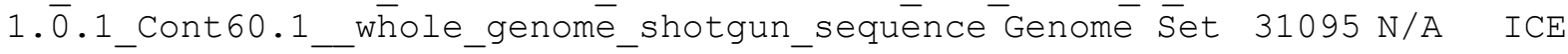

Firmicutes

NZ_HG726025.1_Bacteroides_neonati_strain_MS4_whole_genome_shotgun_sequen $\mathrm{Ce}^{-}$Genome Set 271585 - N/A ICE Bacteroidetés

18048 2_85_Firmicutes_Clostridia_Clostridiales_Peptostreptococcaceae_Clos tridium_.18̄048_2_85.10 Genome Set 93432 N/A ICE Firmicutes

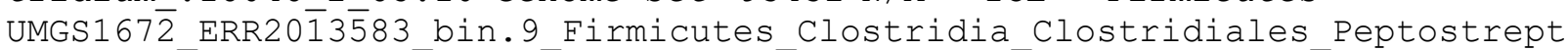

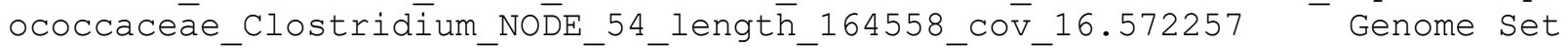
$1645 \overline{5} 8$
N/A ICE Firmicutes

NZ_GL945337.1_Lachnospiraceae_bacterium_2_1_58FAA_genomic_scaffold_superc ont̄1.5_whole_genome_shotgun_sequence Genome Set̄ $278834^{-}$N/A ${ }^{-}$ICE Firmicutes

14207_7_1_Firmicutes_Clostridia_Clostridiales_Lachnospiraceae_Lachnospira $.142 \overline{0} \overline{7}^{-} \overline{7}^{-} 1.3$ Genome set $43 \overline{8596}$ N/A ICE Firmicutes 20298_2_7-Bacteroidetes_Bacteroidia_Bacteroidales_Bacteroidaceae_Bacteroi des_. $20 \overline{2} 9 \overline{8}{ }_{2} 7.5$ Genome ${ }^{-}$Set $560007^{-}$N/A ICE ${ }^{-}$Bacteroidetes 21673_4_18_Firmicutes_Clostridia_Clostridiales_NA_NA_.21673_4_18.36 Genome set $1927 \overline{\bar{\sigma}}$ N/A ICE Firmicutes

14207_7_52_Firmicutes_Clostridia_Clostridiales_Ruminococcaceae_NA_.14207_

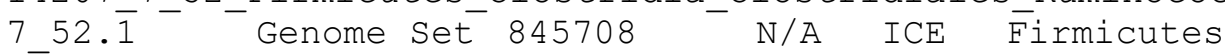

UM̄GS1064_ERR321174_bin.8_Bacteroidetes_Bacteroidia_Bacteroidales_Porphyro monadaceàe_Parabacteroidēs_NODE_3249_lēngth_2890_cov_3.39048 Genome Set $2890^{-} \mathrm{N} / \mathrm{A} \quad$ ICE Bacteroidetes

14207_7_23_Bacteroidetes_Bacteroidia_Bacteroidales_Bacteroidaceae_Bactero ides_.14207 7_23.19 Genome Set $13 \overline{1} 424$ N/A ICE Bacteroidetes UMGS $\overline{9} 20$ SRR $\overline{6} 0 \overline{2} 8474$ bin.9_Firmicutes_Clostridia_Clostridiales_Lachnospirac

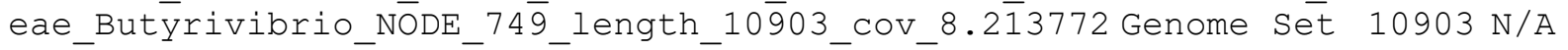

N/A Firmicutes

UMGS1054_SRR5056649_bin.8_Firmicutes_Clostridia_Clostridiales_NA_NA_NODE_

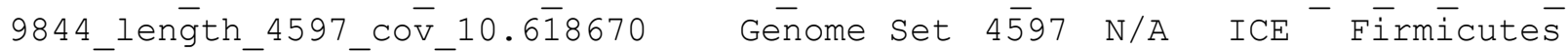

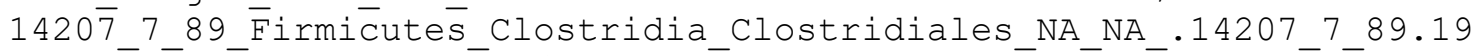

Genome set $6207 \overline{5}$ N/A ICE Firmicutes

NZ_HE578945.1_Enterobacter_massiliensis_JC163_strain_type_strain_JC163 whōle_genome_shotgun_sequence Genome set $\overline{2} 211532 \overline{1} \overline{\mathrm{N}} / \mathrm{A}$ I $\overline{\mathrm{CE}}$ Proteobacteria

20287633 Firmicutes_Clostridia_Clostridiales_Lachnospiraceae_Ruminococc us_.20287 $\overline{6} \_33.4$ Genome Set $104 \overline{028}$ N/A ICE Firmicutes

NZ_GG663524.1_Butyrivibrio_crossotus_DSM_2876_genomic_scaffold_Scfld20_w hole_genome_shotgun_sequence Genome Set $\overline{1} 1174 \overline{4} 8$ N/A ICE Firmicutes 20298_3_71_Eirmicutēs_Clostridia_Clostridiales_NA_NA_.20298_3_71.5

Genome set $9153 \overline{9}$ N/A ICE ${ }^{-}$Firmicutes

UMGS1126_ERR414269_bin.20_Bacteroidetes_Bacteroidia_Bacteroidales_Porphyr omonadacēae_NA_NODE_370_lēength_34268_cov_5.871452 Genome set $34 \overline{2} 68$ N/A ICE Bacteroidetes 
NZ_GG662007.1_Coprococcus_comes_ATCC_27758_genomic_scaffold_Scfld2_whole gēnome shotgün sequence - Genome set $84 \overline{7} 455$ N/A ICE Firmicutes UMMGS122 2 _SRR55799983_bin.22_Firmicutes_Clostridia_Clostridiales_RuminococC

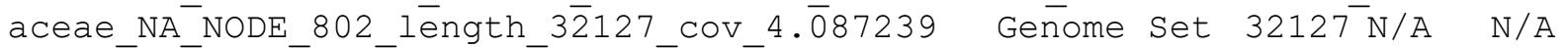

Firmicutes

NZ_GL538344.1_Faecalibacterium_cf._prausnitzii_KLE1255_genomic_scaffold_S

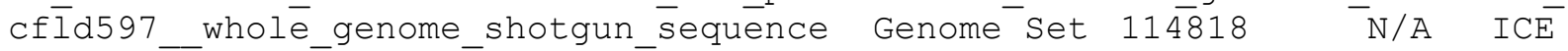
Firmicutes

UMGS918 ERR911961 bin.59 Firmicutes Clostridia Clostridiales NA NA NODE 2 05_length_112838_Cov_27.9334804 Genome Set 112838 N/A $\overline{\mathrm{I} C E}$

Firmicutes

20287_6_37_Firmicutes_Clostridia_Clostridiales_NA_NA_.20287_6_37.9

Genome set $9564 \overline{5}$ N/A ICE ${ }^{-}$Firmicutes

NZ_GL520141.1_Erysipelotrichaceae_bacterium_3_1_53_genomic_scaffold_super coñt1.10_whole_genome_shotgun_sequence Genome $\overline{\text { Set }}{ }^{-} 166748^{-}$N/A ${ }_{\text {ICE }}$ Tenericutes

13470_2_65 Bacteroidetes_Bacteroidia_Bacteroidales Bacteroidaceae Bactero ides_.13470_2_65.1 Genome set $54 \overline{5} 501$ N/A ICE Bacteroidetes $2167 \overline{3}$ 4_5_Bācteroidetes_Bacteroidia_Bacteroidales_Porphyromonadaceae_Barn

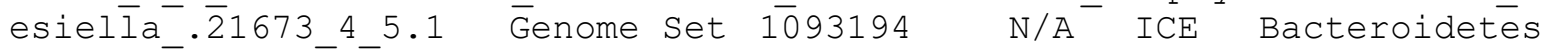
UMGS762_SRR5127̄675_bin.13_Firmicutes_Clostridia_Clostridiales_NA_NA_NODE_

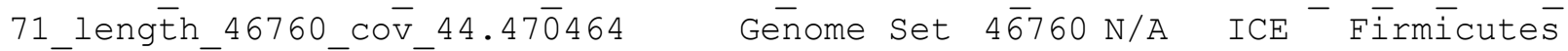
NZ_KI391975.1_St̄rept̄ococcus_sp._2_1_36FAA_genomic_scaffold_supercont2.4 whōle_genome_shotgun_sequence - Genome Set $512 \overline{958}$ N/A ICE

Firmicutes

NZ_DS990120.2_Bacteroides_plebeius_DSM_17135_Scfld_02_3_genomic_scaffold_ _whole_genome_shotgun_sequence Genome set $5116 \overline{1}$ rep_cluster_663 N/A Bacteroidetes

UMGS1469_ERR688640_bin.60_Tenericutes_Mollicutes_Erysipelotrichales_Erysi pelotrichaceae_Solōbacterium_NODE_57_Iength_133550_cov_7.011229

Genome sēt $133550 \quad \bar{N} / A-I C E$ Tenericutes

UMGS528_ERR526064_bin.43_Proteobacteria_Gammaproteobacteria_Aeromonadales _Succinivibrionacēae_NA_NODE_3497_length_7174_COV_3.224189 ${ }^{-}$Genome Set 7174 N/A N/A Proteōbactēria

UMGS1255_ERR1578662_bin.19_Tenericutes_Mollicutes_Erysipelotrichales_Erys

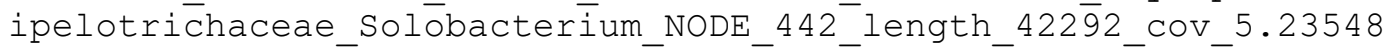

Genome Set 42292 N/A N/A Tenericutes

UMGS261 ERR1190811 bin.41 Firmicutes Clostridia Clostridiales NA NA NODE 118 length 87886 cov_15.631121 Genome set $8 \overline{7886}$ N/A ICE Fírmicutes $134 \overline{1} 4$ 6_47 Firmicutes_clostridia_Clostridiales_Lachnospiraceae_Lachnobact

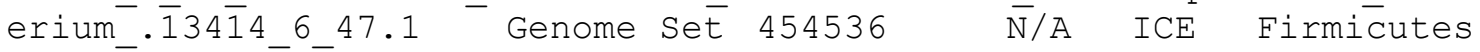
UMGS1 $\overline{6} 98$ ERR $\overline{8} 6 \overline{6} 580$ bin.6_Proteobacteria_Betaproteobacteria_Rhodocyclales_ Rhodocyciaceae_NA_N̄ODE_386_length_5075_cov_2.65837 Genome Set 5075 N/A ICE Proteobacteria

14207_7_83_Firmicutes_Clostridia_Clostridiales_Lachnospiraceae_Roseburia_ $.1420 \overline{7} \overline{7} 8 \overline{3} .13$ Genome Set $127 \overline{496}$ N/A ICE Firmicutes NZ_DS9 $\overline{8} 1 \overline{4} 59.1$ Bacteroides_coprocola_DSM_17136_Scfld_02_32_genomic_scaffol d_whole_genome_shotgun_sequence Genome set $55654 \overline{\mathrm{N}} / \overline{\mathrm{A}} \overline{\mathrm{I}} \mathrm{CE}$

Bacteroidetes

12718_7_92_Firmicutes_Clostridia_Clostridiales_Peptostreptococcaceae_Clos tridium_.127187992.2 Genome SeE 440044 N/A ICE Firmicutes UMGS387_SRR291279̄5_bin.15_Actinobacteria_Actinobacteria_Coriobacteriales_ Coriobacteriaceae_collinsēlla_NoDE_41_length_120776_cov_13.684951

Genome set $\overline{1} 20776 \quad \bar{N} / \mathrm{A} \quad \overline{\mathrm{I}} \mathrm{E}^{-}$Actiñobacteria 
UMGS1683_ERR688506_bin.43_Actinobacteria_Actinobacteria_Coriobacteriales_ Coriobacteriaceae_collinsēlla_NODE_99_length_104584_cov_15.811583

Genome set $\overline{1} 04584 \quad \bar{N} / \mathrm{A}$ ICE $\bar{A}^{-}$Actiñobacteria

UMGS1234 SRR3582140 bin.13 Firmicutes Clostridia Clostridiales NA NA NODE 405_lenḡth_24976_cov_14.49̄0109 Genome Set $24 \overline{9} 76$ N/A ICE Firmicutes $\bar{N} Z$ KQ̄033902.1_Parābactereroides_sp._HGS0025_genomic_scaffold_aczIs-

supercont1.1_whole_genome_shotgun sequence Genome Set $4 \overline{4} 60853$ N/A ICE Bacteroidetes

13414_6_33_Firmicutes_Clostridia_Clostridiales_Lachnospiraceae_NA_.13414 6_33.10- Genome Set 204662 N/A ICE Firmicutes

UMGGS59_ERR589527_bin.14_Firmicutes_Clostridia_Clostridiales_Eubacteriacea

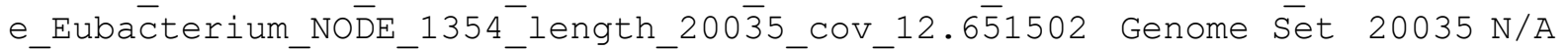
ICE Firmicutes

14207_7_23_Bacteroidetes_Bacteroidia_Bacteroidales_Bacteroidaceae_Bactero ides $.1 \overline{4} 20 \overline{7} 723.49$ Gēnome set $27 \overline{7} 84 \mathrm{~N} / \mathrm{A}$ ICE ${ }_{\text {Bacteroidetes }}$

20298_3_36_Firmicutes_Clostridia_Clostridiales_Clostridiales_Family_XI_Pe

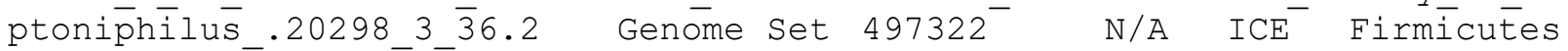
8080_1_78_Firmicutés_Clostridia_Clostridiales_Peptostreptococcaceae_Clost ridium_ERS̄138176.8080_1_78.67-Genome Set 11833 N/A N/A Firmicutes 20298_3_58_Firmicutes_Cİostridia_Clostridiales_NA_NA_.20298_3_58.14 Genome Set $1105 \overline{4} 8$ N/A ICE Firmicutes

NZ_FIZW01000002.1_Murdochiella_massiliensis_strain_SIT12_whole_genome_sh otḡun_sequence Genome Set $1 \overline{293679 \quad \text { N/A }}$ ICE Firmicutes

NZ_GL $\overline{4} 05236.1$ Campylobacter_coli_JV20_genomic_scaffold_SCAFFOLD2_whole_g enōme shotgun sequence Genome Set $28289 \mathrm{~N} / \mathrm{A}$ N/A Prōteobacteria UMGS173_ERR589506_bin.19_Firmicutes_Clostridia_Clostridiales_Eubacteriace

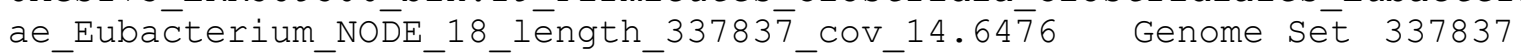

\section{N/A ICE Firmicutes}

14207_7_87_Firmicutes_Clostridia_Clostridiales_NA_NA_.14207_7_87.24

Genome Set $3752 \overline{8}$ N/A N/A Firmicutes

14207_6_12_Bacteroidetes_Bacteroidia_Bacteroidales_Rikenellaceae_Alistipe

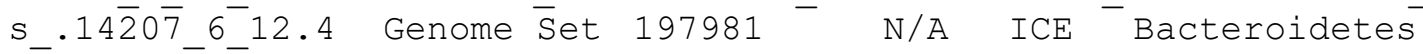

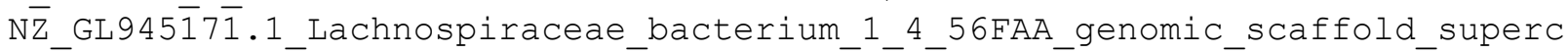

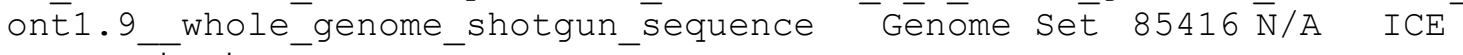
Firmicutes

NZ_LN884299.1_Paenibacillus_ihumii_strain_AT5_whole_genome_shotgun_seque nce Genome Set 4112046 - N/A $\overline{I C E}$ Fírmicutes

13414_6_34_Firmicutes_Clostridia_Clostridiales_Peptostreptococcaceae_Clos tridium_.13̄14_6_34.3- Genome Set 567454 N/A ICE Firmicutes 14207_6_3_Bactēeróidetes_Bacteroidia_Bacteroidales_Porphyromonadaceae_NA_. 14207_6_3.35 Genome Set 22181 N/A ICE Bacteroidetes 20298_2_23_Bacteroidetes_Bacteroidia_Bacteroidales_Bacteroidaceae_Bactero

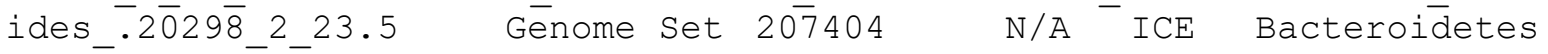
UMGS $\overline{9} 4$ SRR5 $5 \overline{8} 107$ bin.35_Actinobacteria_Actinobacteria_Coriobacteriales_C oriobacteriaceae_Collinsēlla_NODE_156_length_115659_cov_6.943226 Genome Set $115659 \quad \bar{N} / \mathrm{A}^{-}{ }_{\text {ICE }}^{-}$Actiñobactēia

18048_2_67_Firmicutes_Clostridia_Clostridiales_Lachnospiraceae_Blautia_.1 $8048 \overline{2} \overline{6} 7 . \overline{1} 2 \quad$ Genome Set 113960 N/A ICE Firmicutes

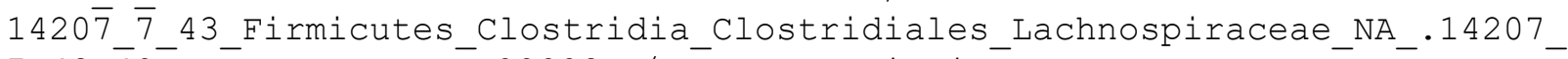
$743 . \overline{4} 2^{-}$Genome Set $22823 \mathrm{~N} / \mathrm{A}^{-}$ICE Firmicutes

UM̄GS1132_ERR1620269_bin.14_Firmicutes_Clostridia_Clostridiales_NA_NA_NODE _121_length_54507_Cov_7.979̄468 Genome set $54 \overline{507}$ N/A ICE Firmicutes 
NZ_GL890520.1_Lachnospiraceae_bacterium_3_1_46FAA_genomic_scaffold_superc

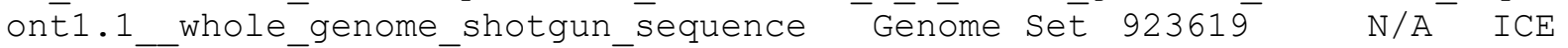
Firmicutes

14207756 Firmicutes Clostridia Clostridiales NA NA .14207 756.19 Genome set $6704 \overline{0}$ N/A ICE ${ }^{-}$Firmicutes

NZ_GL890551.1_Lachnospiraceae_bacterium_6_1_63FAA_genomic_scaffold_superc ont̄1.4_whole_genome_shotgun_sequence Genome Set $331894^{-}$N/A ${ }^{-}$ICE Firmicutes

UMGS359 ERR526036 bin.30 Firmicutes Negativicutes Selenomonadales Veillon

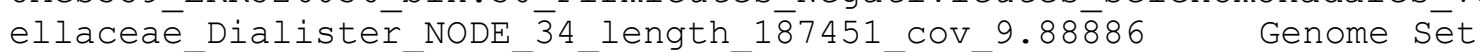
$18 \overline{7} 451$
N/A $\overline{\mathrm{I}} \mathrm{CE}$ Firmicutes

NZ_JH660541.1_Escherichia_sp._4_1_40B_supercont2.3_whole_genome_shotgun_ sequence Genome set $27 \overline{1} 119 \overline{4}$ N/A ICE Proteobacteria

UMGS763 ERR414230 bin.27 Bacteroidetes Bacteroidia Bacteroidales Rikenell aceae_Alistipes_NŌDE_650_length_35963_Cov_5.078061- Genome Set 35963 N/A ICE Bacteroidetes

NZ_GL636615.1_Streptococcus_anginosus_1_2_62CV_genomic_scaffold_supercont $1 . \overline{1}$ _whole_geñome_shotgun_sequence Genome Set $\overline{1137758}$ - N/A $\overline{\text { ICE }}$ Firmicutes

UMGS355_ERR1018193_bin.15_Bacteroidetes_Bacteroidia_Bacteroidales_Bactero

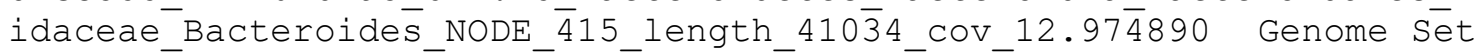
$4 \overline{1} 034 \mathrm{~N} / \mathrm{A} \quad \mathrm{IC} \overline{\mathrm{E}}$ Bācteroidetes

NZ_GL834360.1_Clostridium_symbiosum_WAL-

146̄73_genomic_scaffold_supercont1.4_whole_genome_shotgun_sequence Genome set $62841 \overline{3} \quad$ N/A ICE Firmicutes

21673_4_18_Firmicutes_Clostridia_Clostridiales_NA_NA_.21673_4_18.25 Genome set $3713 \overline{6}$ N/A ICE ${ }^{-}$Firmicutes

UMGS722_ERR1297657_bin.16_Actinobacteria_Actinobacteria_Coriobacteriales_ Coriobacteriaceae_Collinsēlla_NODE_463_lēngth_19232_cov_3.599990

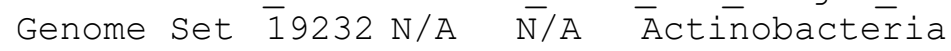

UMGS2059_ERR1297781_bin.12_Actinobacteria_Actinobacteria_Coriobacteriales _Coriobacteriaceae_Collinsēlla_NODE_1306_İength_5134_cov_1.744241

Genome set $5 \overline{1} 34$ N/A N/A Actinobacteria

12718_7_72_Firmicutes_Clostridia_Clostridiales_NA_NA_.12718_7_72.10

Genome set $1063 \overline{3} 4 \quad$ N/A ICE Firmicutes

NZ_CTEJ01000002.1_Lascolabacillus_massiliensis_strain_SIT8_whole_genome_ shōtgun_sequence Genome set $1664 \overline{769}$ N/A ICE Bäteroidetes

13470_2_81_Firmicutes_Clostridia_Clostridiales_Lachnospiraceae_Blautia_.1 3470_ $\overline{2} \overline{8} 1 . \overline{5} 5$ Genome set $155 \overline{16} \mathrm{~N} / \mathrm{A}$ ICE Firmicutes

UMGS $33 \overline{9}$ ERR688601_bin.50_Tenericutes_Mollicutes_Erysipelotrichales_Erysip elotrichaceae_Solōbacterīum_NODE_139_length_592 $\overline{6} 2$ _cov_20.122553

Genome Set 59262 N/A ${ }^{-}$N/A - Tenericutes

13470_2_67_Bacteroidetes_Bacteroidia_Bacteroidales_Porphyromonadaceae_Par abactēeroides_.13470_2_67.1 Genome set 1084885 N/A ICE Bacteroidetes

NZ_JH370372.1_Alistipes_indistinctus_YIT_12060_genomic_scaffold_supercont $1 . \overline{2}$ _whole_geñome_shotgün_sequence Genome set $\overline{1180242}$ - N/A $\overline{\text { ICE }}$ Bacteroidetes

14207_7_90_Firmicutes_Clostridia_Clostridiales_NA_NA_.14207_7_90.24 Genome set $5633 \overline{2}$ N/A ICE Firmicutes

12718 7_29 Bacteroidetes_Bacteroidia_Bacteroidales_Bacteroidaceae Bactero ides_.1 $1 \overline{2} 71 \overline{8}$ 7_29.4 Genome set $63 \overline{6} 223$ N/A ICE Bacteroīetes 1341 $\overline{4}$ 6_33_Firmicutes_Clostridia_Clostridiales_Lachnospiraceae_NA_.13414_ 6_33.15 ${ }^{-}$Genome Set 81687 N/A ${ }^{-}$ICE Firmicütes 
UMGS1254_SRR5056823_bin.4_Actinobacteria_Actinobacteria_Coriobacteriales_

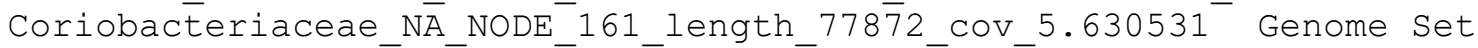

$77872 \mathrm{~N} / \mathrm{A} \quad \overline{\mathrm{N}} / \mathrm{A}^{-}$Act̄inob̄acteriā

20298_3_64_Firmicutes_Clostridia_Clostridiales_Ruminococcaceae_NA_.20298_ $364 . \overline{8}-$ Genome Set $171063-$ N/A ICE Firmicutes

NZ̄_CP009244.1_Corynebacterium_ammoniagenes_DSM_20306_strain_9.6_complete gēenome Genome set $279018 \overline{5} \quad$ N/A ICE Áctinobacteriā

UMGS1673_ERR1578622_bin.15_Firmicutes_Clostridia_Clostridiales_Peptostrep

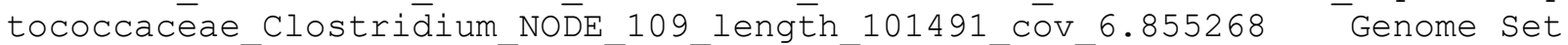
$10149 \overline{1} \quad$ N/A $\bar{N} / A \quad$ Firmicutes

UMGS709_SRR2992911_bin.12_Firmicutes_Clostridia_Clostridiales_Oscillospir

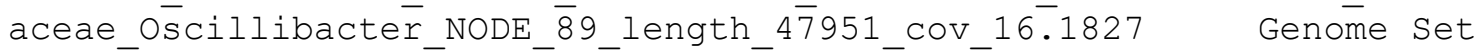

$47951 \mathrm{~N} / \mathrm{A} \quad I C \bar{E} \quad$ Firmícutes

UMGS1389_ERR1620298_bin.4_Firmicutes_Clostridia_Clostridiales_NA_NA_NODE_

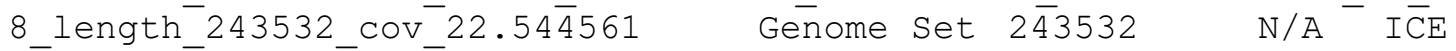

Firmicutes

NZ_GG703826.1_Providencia_rustigianii_DSM_4541_Scfld9_whole_genome_shotg un sequence Gēnome set $14 \overline{7} 18 \mathrm{~N} / \mathrm{A} \quad \mathrm{N} / \mathrm{A}^{-}$Proteob̄acteria

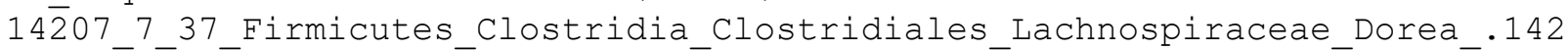
07 7_ $37.28^{-}$Genome Set 50659 N/A ${ }^{-}$ICE Firmicütes

UMGGST1425_ERR1190839_bin.22_Firmicutes_Clostridia_Clostridiales_Peptostrep

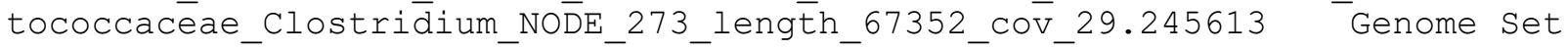
$67352 \bar{N} / \mathrm{A} \quad \mathrm{N} / \mathrm{A} \quad \overline{\mathrm{F}}$ irmicutes

UMGS1243_SRR5558136_bin.38_Firmicutes_Clostridia_Clostridiales RuminococC aceae Ruminococcus N

\section{N/A Plasmid Firmicutes}

21673_4_19_Firmicutes_Clostridia_Clostridiales_Lachnospiraceae_Blautia_.2

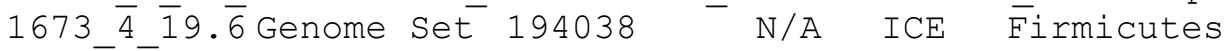

$2167 \overline{3}$ 4__33_Bacteroidetes_Bacteroidia_Bacteroidales_Porphyromonadaceae_Par abacteroides_.21673_4_33.16 Genome set 142305 N/A ICE

Bacteroidetes

NZ_FLKP01000001.1_Christensenella_timonensis_strain_Marseille-

P2 437 _whole_genome_shotgun_sequeñce Genome set 336694 N/A ICE Firmicutes

UMGS1113 SRR5580042 bin.20 Firmicutes Clostridia Clostridiales NA NA NODE 271_lenḡth_66560_cov_8.986̄222 Genome set $66 \overline{5} 60$ N/A ICE Firmicutes 20298_3_35_Firmicutes_Clostridia_Clostridiales_Lachnospiraceae_Blautia_.2 0298 3 $35 . \overline{2} 6 \quad$ Genome Set $463 \overline{13}$ N/A N/A Firmicutes

UMGST̄011_ERR414329 bin.39 Firmicutes_Clostridia_Clostridiales_NA_NA NODE 352 length 31600 Cov_4.645681 Geñome Set $3 \overline{1} 600$ N/A N/A Firmícutes UMG $\bar{S} 182$ ERR $63635 \overline{1}$ _biñ.83 Bacteroidetes_Bacteroidia_Bacteroidales_Bacteroi daceae_Bacteroides_NODE_410_length_736833_cov_19.574 469 Genome Set

$\overline{73683} \mathrm{~N} / \mathrm{A} \quad \mathrm{IC} E$ Bactēeroidetēs

NZ_LN908986.1_Eggerthellaceae_bacterium_AT8_genome_assembly_Hugonella_mas siliensis_scäffold_scaffold00̄009_wholēe_geñome_shōtgun_sequence

Genome set $14 \overline{7} 5894 \quad$ N/A $\bar{I} C E$ Actinobācteria

14207_6_13_Bacteroidetes_Bacteroidia_Bacteroidales_Bacteroidaceae_Bactero ides_.14207 6_13.8 Genome Set 124262 N/A ICE Bacteroidetes 11861_6_58_Firmicutes_Clostridia_Clostridiales_Lachnospiraceae_Blautia_.1 $1861 \overline{6} \overline{5} 8 . \overline{6}$ Genome Set $254968-$ N/A ICE Firmicutes UMGST1981_SRR1761711_bin.3_Firmicutes_Clostridia_Clostridiales_NA_NA_NODE

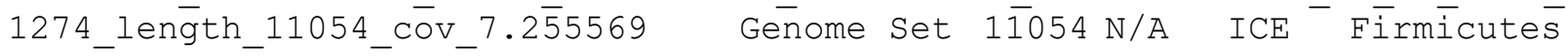


UMGS219 ERR321453 bin.7 Bacteroidetes Bacteroidia Bacteroidales Bacteroid

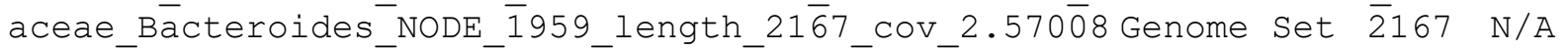
N/A Bacteroidetes

NZ FCEX01000010.1 Peptoniphilus phoceensis strain SIT15 whole genome sho tgün_sequence Genome Set $210464 \quad \mathrm{~N} / \overline{\mathrm{A}}$ ICE Firmicutes

13414 6_50_Firmicutes_Clostridia_Clostridiales_Lachnospiraceae_Dorea_.134 14 6_50.4- Genome Set 224182 N/A ICE Firmicutes

NZ_GG703824.1_Providencia_rustigianii_DSM_4541_Scfld7_whole_genome_shotg un sequence Genome Set $34 \overline{8} 09$ N/A Plasmid Proteobacteria

20298_2_28_Firmicutes_Clostridia_Clostridiales_NA_NA_.20298_2_28.8

Genome Set $1253 \overline{8} 5 \quad$ N/A ${ }^{-}$ICE Firmicutes

UMGS1833_SRR5963345_bin.25_Firmicutes_Clostridia_Clostridiales_Lachnospir

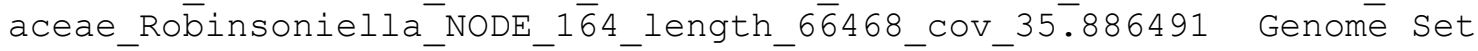
$66468 \mathrm{~N} / \mathrm{A} \quad \mathrm{IC} \overline{\mathrm{E}}$ Firmicutes

UMGS1383_SRR5558252_bin.31_Tenericutes_NA_NA_NA_NA_NODE_89_length_68031_C

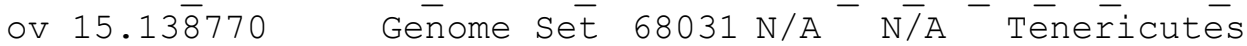

21673_4_3_Firmicutes_Clostridia_Clostridiales_Lachnospiraceae_NA_.21673_4 3.5 Genome Set $179 \overline{9} 99 \quad$ N/A ICE Firmicutes

$\bar{N} Z$ _FCEX01000008.1_Peptoniphilus_phoceensis_strain_SIT15_whole_genome_sho

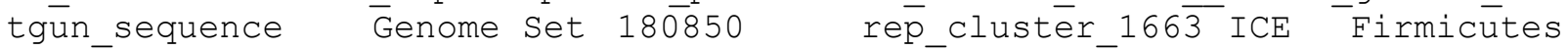
NZ_GĞ669059.1_Enterococcus_faecium_TX1330_genomic_sčaffold_SCAFFOLD3_who le_genome_shotgun_sequence Genome- set $1 \overline{7} 4673$ - rep_clüster_893 ICE

Firmicutes

UMGS200_SRR6028624_bin.16_Firmicutes_Clostridia_Clostridiales_Ruminococca

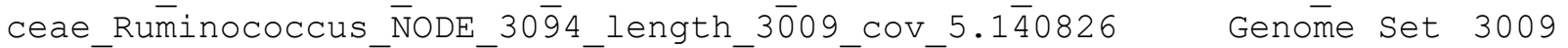
N/A Plasmid
Firmicutes

UMGS1553_ERR1190927_bin.14_Firmicutes_Clostridia_Clostridiales_Peptostrep

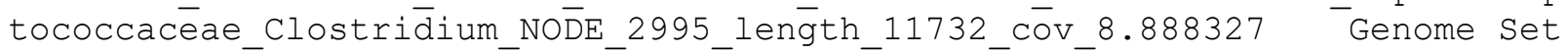
$11732 \bar{N} / \mathrm{A} \quad \mathrm{N} / \mathrm{A} \quad \overline{\mathrm{F}} \mathrm{irmi}$ cutes

NZ_JH590968.1_Erysipelotrichaceae_bacterium_6_1_45_genomic_scaffold_super coñt1.1_wholèegenome_shotgun_sequence Genome $\overline{\text { Set }}{ }^{-145736 \bar{\sigma}^{-}}$N/A ${ }^{-}$ICE Tenericutes

UMGS2027_SRR1761721_bin.40_Bacteroidetes_Bacteroidia_Bacteroidales_Prevot

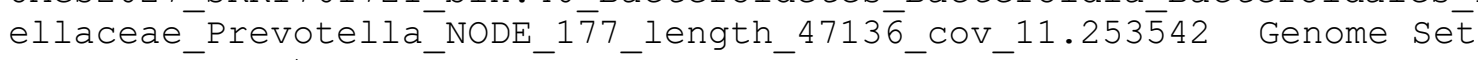

$47 \overline{13} 6 \mathrm{~N} / \mathrm{A} \quad \mathrm{ICE} \quad$ Bäcteroidetes

20287_6_22_Firmicutes_Clostridia_Clostridiales_Ruminococcaceae_Ruminococc us_.20287_ $\overline{6} 22.5$ Genome Set $165 \overline{189}$ N/A ICE Firmicutes

18048_1_69 $\overline{9}$ Firmicutes_Clostridia_Clostridiales_Lachnospiraceae_NA_.18048_ 1_69.3 ${ }^{-}$Genome Set $27559 \mathrm{~N} / \mathrm{A}^{-}$ICE Firmicutes

UMGGS1099_ERR526047_bin.23_Firmicutes_Clostridia_Clostridiales_NA_NA_NODE_ 1229_lenḡth_20502_Cov_11.842324 Genome Set $2 \overline{0502}$ N/A ICE Firmícutes UMGST̄1927_ERR $86660 \overline{1}$ biñ.6_Bacteroidetes_Bacteroidia_Bacteroidales_Prevotel laceae_Prevotella_N̄ODE_3_length_89786_Cov_6.75384 Genome set $8 \overline{9} 786$ N/A

ICE Bacteroidetes

13470_2_92_Firmicutes_Clostridia_Clostridiales_Lachnospiraceae_NA_.13470_ 2 292. $\overline{9}^{2}-$ Genome Set $121460-$ N/A ICE Firmicutes

NZ̄_JH376470.1_Pseudomonas_sp._2_1_26_genomic_scaffold_supercont1.10_whol e_genome_shotgun_sequence Geñme Set $2386 \overline{8} 2$ N/A ICE

Proteobacteria

UMGS208_ERR525989_bin.4_Bacteroidetes_Bacteroidia_Bacteroidales_Porphyrom onadaceāe_Parabactereroidès_NODE_83_lenḡth_109882_cō_17.4494 Genome Set $109 \overline{882}$ N/A ICE Bäctēroidetes 
NZ_GL891980.1_Dysgonomonas_gadei_ATCC_BAA-

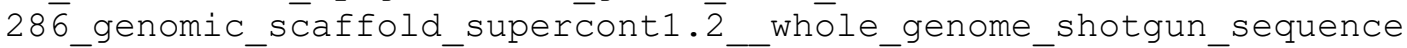

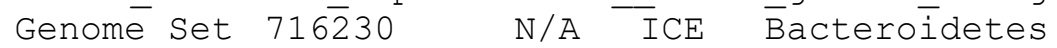

UMGS530 SRR2155369 bin.7 Firmicutes Clostridia Clostridiales NA NA NODE 5

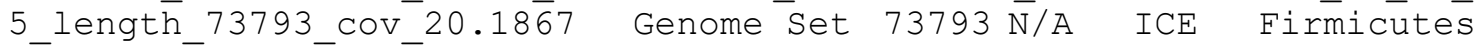
UM̄GS174_SRR1761690_bin.37_Firmicutes_Clostridia_Clostridiales_Ruminococca ceae_NA_NODE_34_length_1334449_cov_98.451197 Genome set $1334 \overline{4} 9$ N/A I $\overline{C E} \quad \overline{\text { Firmicutes }}$

20298363 Bacteroidetes Bacteroidia Bacteroidales Rikenellaceae Alistipe S_.20298_3_63.1 Genome Set 819193 N/A ICE Bacteroidetes

UMGGS1710_ERR1293845_bin.21_Bacteroidetes_Bacteroidia_Bacteroidales Bacter

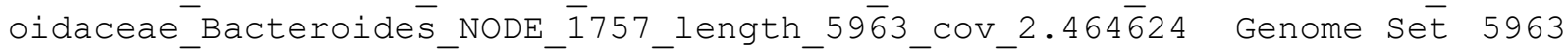

N/A N/A Bacteroidetes

UMGS35_ERR525726_bin.20_Firmicutes_Clostridia_Clostridiales_Peptostreptoc

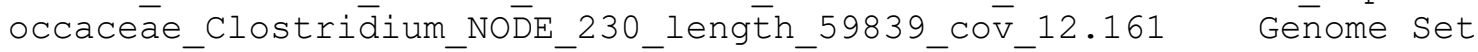

$59 \overline{8} 39 \mathrm{~N} / \mathrm{A}$ ICE Firmicutes

14207_7_1_Firmicutes_Clostridia_Clostridiales_Lachnospiraceae_Lachnospira $.142 \overline{0} \overline{7}^{-}{ }^{-} 1.13$ Genome Set $46 \overline{62} 4 \mathrm{~N} / \mathrm{A}$ N/A Firmicutes

UMGS1852_ERR414343_bin.75_Firmicutes_Clostridia_Clostridiales_Peptostrept

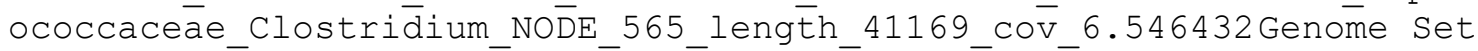

$4116 \overline{9}$ N/A Plasmid Firmicutes

UMGS897_SRR2155371_bin.22_Firmicutes_Clostridia_Clostridiales_NA_NA_NODE_

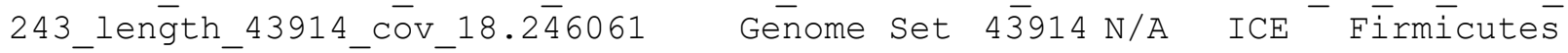
1420̄76_13_Bacter $\overline{0}$ -

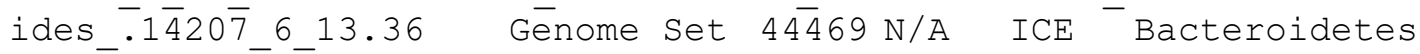

UMGS $\overline{8} 35$ ERR $\overline{5} 8 \overline{9} 530$ bin.45_Bacteroidetes_Bacteroidia_Bacteroidales_Porphyro monadacēae_NA_NODE_18_length_129018_cov_8.073176-Genome Set $1 \overline{29018}$

$\mathrm{N} / \mathrm{A}-\mathrm{ICE}$ Bäcteroidetés

11861_6_63_Bacteroidetes_Bacteroidia_Bacteroidales_Rikenellaceae_Alistipe

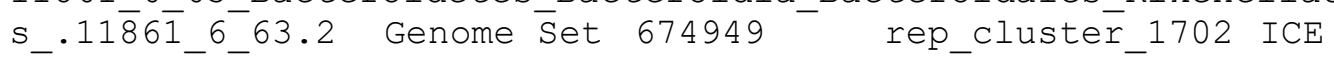

Bacteroidetes

UMGS629_SRR5580103_bin.48_Actinobacteria_Actinobacteria_Coriobacteriales_

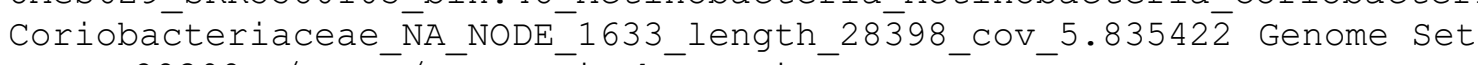

$28398 \mathrm{~N} / \mathrm{A} \quad \overline{\mathrm{N}} / \mathrm{A}^{-}$Act̄inobācteria

UMGS131_ERR1305881_bin.48_Firmicutes_Clostridia_Clostridiales_Ruminococca

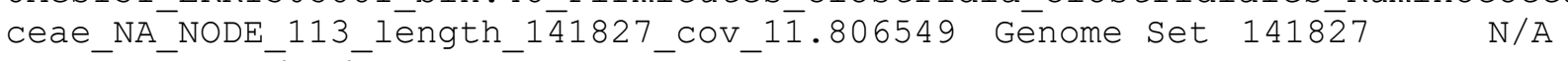

ICE Firmicutes

UMGS1298_ERR1018268_bin.53_NA_NA_NA_NA_NA_NODE_562_length_42968_Cov_5.677 883 Genome set $42 \overline{9} 68 \mathrm{~N} / \mathrm{A}^{-} \overline{\mathrm{ICE}}-{ }^{-} \overline{\mathrm{NA}}$

NZ_KE150405.1_Lachnospiraceae_bacterium_3_1_57FAA_CT1_acsNU-

supercont1.1_whole_genome_shotgun_sequence Genome Set 4263893 N/A ICE Firmicutes

NZ_DS990450.1_Helicobacter_pullorum_MIT_98-

548̄9_supercont2.10_genomic_scaffold__whöle_genome_shotgun_sequence Genome Set $5 \overline{7} 37 \mathrm{~N} / \mathrm{A}$ N/A Proteobacteria

14207 6_9_Bacteroidetes_Bacteroidia_Bacteroidales_Bacteroidaceae_Bacteroi des_. $\overline{1} 4 \overline{2} 0 \overline{7}$ 6_9.29Genome ${ }^{-}$Set 57155 N/A ICE Bacteroidetes

UMGSיS2019_ERRT505098_bin.42_Firmicutes_Clostridia_Clostridiales_Peptostrept

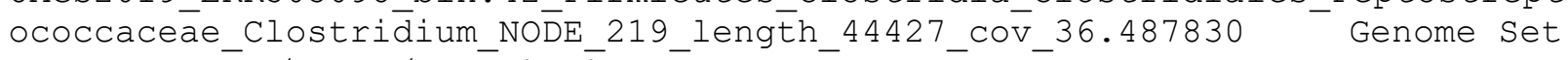
44427 N/A N/A Firmicutes

12718_7_92_Firmicutes_Clostridia_Clostridiales_Peptostreptococcaceae_Clos tridium_.12718_7_92.7 Genome Set 188262 N/A ICE Firmicutes 
NZ_DS572690.1_Streptococcus_infantarius_subsp._infantarius_ATCC_BAA$10 \overline{2}$ Scfld_02_ī6_genomic_scafifold_whole_genome_shotgun_sequence Genome Set $83420 \bar{N} / A \quad$ ICE Firmicutes

NZ KE150435.1 Coprococcus sp. HPP0074 genomic scaffold acAqDsupercont1.1_whole_genomé_shötgun_sequence Genome sēt 1814818 N/A ICE Firmicutes

NZ_JH414887.1_Campylobacter_sp._10_1_50_genomic_scaffold_supercont1.1_wh olē_genome_shōtgun_sequence Genome $\overline{S e t}^{-}$- $50648^{-}$N/A ICE

Protéobacterīa

13414_6_70_Firmicutes_Clostridia_Clostridiales_Lachnospiraceae_NA_.13414_ $670 . \overline{8}-$ Genome Set 158140 N/A ICE Firmicutes

14̄207_7_18_Firmicutes_Clostridia_Clostridiales_NA_NA_.14207_7_18.3 Genome set $4211 \overline{8} 3 \quad$ N/A ICE Firmicutes

20298_3_19_Proteobacteria_Gammaproteobacteria_Enterobacteriales_Enterobac

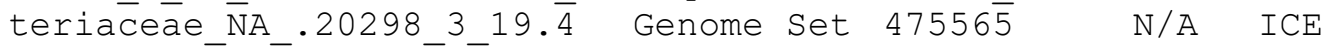

Proteobacteria

NZ_JH599902.1_Coprobacillus_sp._8_2_54BFAA_genomic_scaffold_supercont1.2_ _whole_genome_shotgun_sequence $-{ }^{-}$Genome set $1415 \overline{6} 81$ N/A ICE Tenericutes

UMGS365_SRR5558121_bin.15_Firmicutes_Clostridia_Clostridiales_NA_NA_NODE_ 16_length_248977_Cō_7.913̄210 Geñome Set $2 \overline{48977}$ N/A I $\overline{C E}$

Firmicutes

NZ_GL883856.1_Paraprevotella_xylaniphila_YIT_11841_genomic_scaffold_Scfld $10 \overline{3}$ _whole_geñome_shotgun_sequence Genome set $7064 \overline{9}$ N/A ICE Bacteroidetes

20287_6_6_Proteobacteria_Gammaproteobacteria_Enterobacteriales_Enterobact

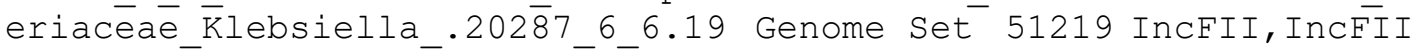

Plasmid Proteobacteria

NZ_EQ973638.1_Bacteroides_coprophilus_DSM_18228_=_JCM_13818_genomiC_scaff old__Scfld10_whole_genome_shotgun_sequence $\overline{\text { w }}$ Genome $\overline{\text { Set }} 11 \overline{3} 452$ - N/A ICE Bacteroidetes

12718_7_72_Firmicutes_Clostridia_Clostridiales_NA_NA_.12718_7_72.15 Genome Set $5214 \overline{6}$ N/A ICE ${ }^{-}$Firmicutes

13470_2_91_Firmicutes_Clostridia_Clostridiales_Lachnospiraceae_NA_.13470_ 2 2 $91 . \overline{3}-$ Genome Set 208884 N/A ICE Firmicutes

2̄1673_4_87_Firmicutes_Clostridia_Clostridiales_Lachnospiraceae_Dorea_.216 73 4_87.11 Genome Set 91588 N/A ICE Firmicutes

14 $\overline{2} 0 \overline{7}$ 7_18_Firmicutes_Clostridia_Clostridiales_NA_NA_.14207_7_18.4 Genome set $3378 \overline{4} 9 \quad$ N/A

21673_4_5_Bacteroidetes_Bacteroidia_Bacteroidales_Porphyromonadaceae_Barn

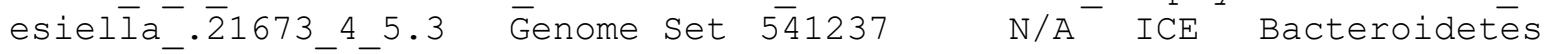
NZ_CYUO $0100000 \overline{5} . \overline{1}$ _Peptoniphilaceae_bacterium_SIT14_genome_assembly_Lagier elīa_massiliensis_contig_contig00005_whole_genomé_shotgūn_sequence Genome Set $\overline{1068061}$ N/A ICE Firmicutes

NZ_KI271097.1_Eubacterium_ramulus_ATCC_29099_genomic_scaffold_Scaffold134 2_whole_genome_shotgun_séquence Genome Set 548 N/A Plasmid Firmicutes

NZ_GL870812.1_Clostridium_sp._D5_genomic_scaffold_supercont1.4_whole_gen omé_shotgun_séquence Geñome ${ }^{-} \bar{t}^{-} 402542^{-}$N/A ${ }^{-}$ICE Firmicutes 1271̄8_7_84_Firmicutes_Clostridia_Clostridiales_Ruminococcaceae_NA_.12718_ $784 . \overline{1} 2^{-}$Genome Set 38018 N/A ${ }^{-}$ICE Firmicutes UM̄GS2007_ERR209576_bin.9_Bacteroidetes_Bacteroidia_Bacteroidales_Bacteroi

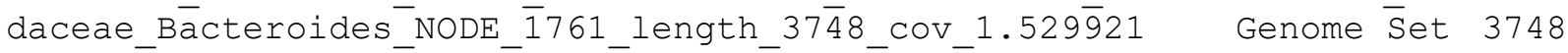
$\overline{\mathrm{N}} / \mathrm{A} \quad \mathrm{ICE} \quad \overline{\mathrm{B}} \mathrm{actero} \overline{\mathrm{A}} \mathrm{det} \overline{\mathrm{e}} \mathrm{s}$ 
UMGS1697_SRR5580035_bin.43_Bacteroidetes_Bacteroidia_Bacteroidales_Bacter

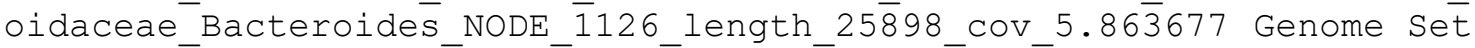

$25 \overline{8} 98 \mathrm{~N} / \mathrm{A} \quad \mathrm{ICE}^{-}$Bacteroìidetes

13414_6_43_Firmicutes_Clostridia_Clostridiales_Ruminococcaceae_NA_.13414 $643 . \overline{3}-$ - Genome Set 273585 - N/A ICE Firmicutes

UMMGS220 SRR5057033 bin.5 Firmicutes Clostridia Clostridiales Ruminococcac

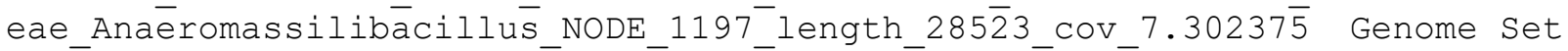
28523 N/A N/A Firmicutes

NZ_GL945020.1_Bacteroides_ovatus_3_8_47FAA_genomic_scaffold_supercont1.3_ -whole_genome_shotgun_sequence $-\bar{G}$ - $\overline{\text { nome Set }} 8014 \overline{65} \quad \mathrm{~N} / \overline{\mathrm{A}}$ ICE Bacteroidetes

21673_4_87_Firmicutes_Clostridia_Clostridiales_Lachnospiraceae_Dorea_.216 73_4_87.4- Genome Set 238478 N/A ICE Firmicutes

NZ_EQ 973344.1 Clostridium_methylpentosum_DSM_5476_Scfld6_genomic_scaffold whole_genoméeshotgun_sequence Genome Set $^{-} 156 \overline{0} 487$ N/A I $\bar{C} E$ Firmicutes

UMGS1638_SRR5127671_bin.14 Actinobacteria_Actinobacteria_Coriobacteriales _Coriobacteriaceae_Collinsēlla_NODE_775_lēength_12057_cov_5.324779

Genome set $1 \overline{2} 057$ N/A ICE ACtinobactería

21673_4_19_Firmicutes_Clostridia_Clostridiales_Lachnospiraceae_Blautia_.2 $1673 \overline{4} \overline{1} 9 . \overline{2}$ Genome set 371155 N/A ICE Firmicutes

UMGS $\overline{6} 4 \overline{6}$ ERR5 89578 bin.21_Firmicutes_Clostridia_Clostridiales_Ruminococcac

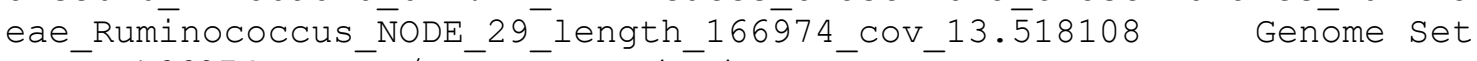
$166974 \quad$ N/A I $\bar{C} E$ Firmicutes

20287_6_37_Firmicutes_Clostridia_Clostridiales_NA_NA_.20287_6_37.8 Genome Set $1067 \overline{0} 7$ N/A ICE Firmicutes

UMGS285_SRR1201570_bin.8_Firmicutes_Clostridia_Clostridiales_NA_NA_NODE_2 8 length̄ 135093 cov_9.26101 Genome Set 135093 N/A ICE Firmicutes

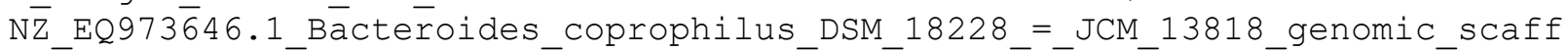
old__Scfld18_whole_genome_shotgun_sequence Genome Set 400038 N/A ICE Bacteroidetes

NZ_GL892005.1_Dysgonomonas_mossii_DSM_22836_genomic_scaffold_supercont1.2 whole_genoméeshotgun_sequence Genome Set $80369 \overline{1}$ N/A ICE Bacteroīetes

14207_7_59_Firmicutes_Clostridia_Clostridiales_NA_NA_.14207_7_59.8 Genome Set $1594 \overline{6} 9 \quad$ N/A ICE Firmicutes

UMGS945_ERR1620269_bin.39_Firmicutes_Clostridia_Clostridiales_Peptostrept

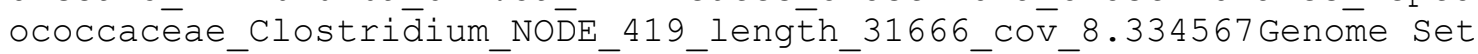
$3166 \overline{6} \mathrm{~N} / \mathrm{A} \quad \mathrm{ICE}$ Firmicutēs

NZ_GL945019.1_Bacteroides_ovatus_3_8_47FAA_genomic_scaffold_supercont1.2_ _whole_genome_shotgun_sequence - Genome Set $9117 \overline{9} 4 \quad \mathrm{~N} / \overline{\mathrm{A}}$ ICE Bacteroidetes

UMGS1126_ERR414269_bin.20_Bacteroidetes_Bacteroidia_Bacteroidales_Porphyr

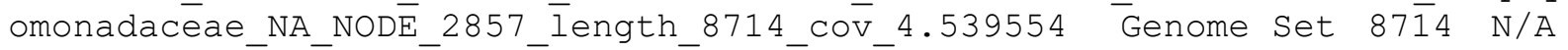
ICE Bacteroidetes

UMGS1529_SRR5279269_bin.27_Firmicutes_Clostridia_Clostridiales_Eubacteria

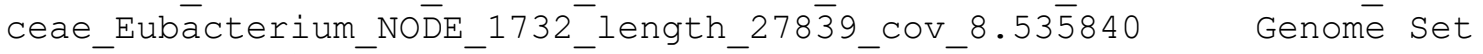
$27839 \mathrm{~N} / \mathrm{A}$ ICE Firmicutes

UMGS1156_ERR1620345_bin.42_Firmicutes_Clostridia_Clostridiales_Lachnospir aceae_Blautia_NODE_437_length_42843_cov_3.484084 Genome Set 42843 N/A ICE Firmicutes

NZ_LN908961.1_Ruminococcaceae_bacterium_AM2_genome_assembly_Fournierella_ massiliensis_scaffold_scaffoldd00016_whole_genome_shotgun_sequence Genome $\overline{\text { Set }} 39695 \overline{8} \quad$ N/A ICE Firmicutes 
UMGS1844_ERR589567_bin.9_Tenericutes_Mollicutes_Erysipelotrichales_Erysip elotrichāceae_Solob̄acterīum_NODE_408_length_4937̄6_cov_4.853369 Genome Set $49376 \mathrm{~N} / \overline{\mathrm{A}}$ ICE Tenericutes

21673_4_37_Firmicutes_Clostridia_Clostridiales_Lachnospiraceae_NA_.21673_ $437 . \overline{6}-$ Genome Set 186715 - N/A ICE Firmicutes

UMGGS777_ERR1305885_bin.21_Eirmicutes_Clostridia_Clostridiales_NA_NA_NODE_ 91_length_86914_cō_ $8.800 \overline{6} 31$ Genome set 86914 N/A ICE Firmicutes

UMGS1105 SRR1214948 bin.2 Bacteroidetes Bacteroidia Bacteroidales Prevote

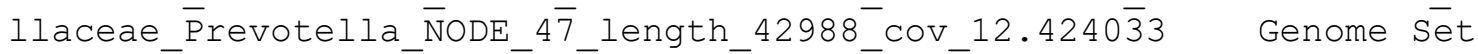

$4 \overline{2} 988$ N/A ICE Bacteroidē̄es

NZ_JH414888.1_Campylobacter_sp._10_1_50_genomic_scaffold_supercont1.2_wh olēegenome_shōtgun sequence Geñome Set $832312-$ N/A ${ }^{-}$ICE

Proteobactería

UMGS40 SRR2912796 bin.41 Firmicutes Clostridia Clostridiales Ruminococcac eae_Ruminococcus_NoDE_30_length_1270̄87_cov_19.5̄59893 Genōme Set

$$
127087 \quad \text { N/A I } \overline{C E} \text { Firmicutes }
$$

NZ_ACCL02000006.1_Marvinbryantia_formatexigens_DSM_14469_B_formatexigens-

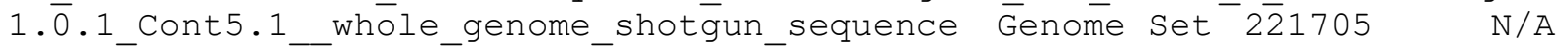
ICE Firmicutes

UMGS698_ERR414252_bin.16 Bacteroidetes Bacteroidia Bacteroidales Bacteroi daceae_Bacteroides_NODE_3744_length_8979_Cov_44.549305 Genome Set 8979 $\overline{\mathrm{N}} / \mathrm{A} \quad \mathrm{ICE} \quad \overline{\mathrm{B}} \mathrm{C}$ cteroidetès

20298_3_13_Proteobacteria_Gammaproteobacteria_Enterobacteriales_Enterobac teriaceàe_K̄lebsiella_.20298_3_13.7Genome set 403182 N/A ICE Proteobacteria

UMGS1425_ERR1190839_bin.22_Firmicutes_Clostridia_Clostridiales_Peptostrep

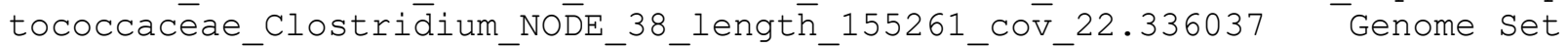
$15526 \overline{1} \quad$ N/A N $/ A \quad$ Firmicutes

UMGS564_SRR2726136_bin.31_Firmicutes_Clostridia_Clostridiales_Lachnospira

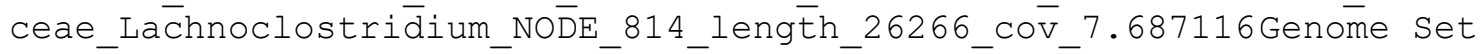

$26266 \mathrm{~N} / \mathrm{A} \quad \mathrm{ICE}$ Firmicutēs

NZ_KE150407.1_Lachnospiraceae_bacterium_3_1_57FAA_CT1_acsNU-

supercont1.3_whole_genome_shōtgun_sequēnce ${ }^{-}$Genome Set 1161241 N/A ICE Firmicutes

UMGS12_SRR5106488_bin.11_Proteobacteria_Betaproteobacteria_Burkholderiale

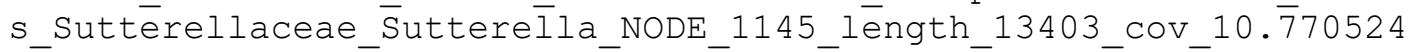

Genome Set 13403 N/A ICE Proteobacteria

NC_021039.1_Ruminococcus_champanellensis_type_strain_18P13T_draft_genome Genome set $2573208^{-} \quad$ N/A ICE Firmicutes

NZ_HE978600.1_Brevibacterium_sp._JC43_genomic_scaffold_scaffold00001_wh olēegenome_shōtgun_sequence Genome sēt $65685 \overline{4} \quad \mathrm{~N} / \overline{\mathrm{A}}$ ICE Actinobacteria

UMGS308_SRR4305073_bin.1_Actinobacteria_Actinobacteria_Coriobacteriales_C oriobact̄eriaceae_Cōllinsēella_NODE_2_lenğth_160609_cov_ž25.182281

Genome set $160609 \quad \bar{N} / \mathrm{A}-\overline{I C E}$ Actinobacteriā

NZ_LN909059.1_Paenibacillus_sp._MT18_genome_assembly_Paenibacillus_rubiin fantis_scafföld_scaffold00013_whole_genomé_shotgun_sequence Genome set $158767 \quad$ N/A ICE Firmicutes

NZ_KE993547.1_Clostridium_sp. ATCC_29733_genomic_scaffold_Scaffold163_wh olē_genome_shōtgun_sequence Genome Set $\overline{7} 0968$ N/A $\bar{A}$ ICE $\overline{\text { Firmicutes }}$ UMGST 1684 ERRR011192_bin.15_Bacteroidetes_Bacteroidia_Bacteroidales_Prevote

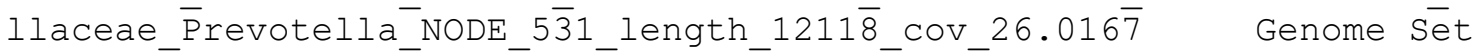

$1 \overline{2} 118 \mathrm{~N} / \mathrm{A} \quad \mathrm{IC} E$ Bactēroidetēs 
11861_6_58_Firmicutes_Clostridia_Clostridiales_Lachnospiraceae_Blautia_.1 $1861 \overline{6} \overline{5} 8 . \overline{1} 3$ Genome set $865 \overline{86}$ N/A ICE Firmicutes UMGST1833_SRR5963345_bin.25_Firmicutes_Clostridia_Clostridiales_Lachnospir aceae Rob̄insoniella NODE $1 \overline{2} 1$ length $9 \overline{2} 772$ Cov 35.719046 Genome Set

\section{$92772 \mathrm{~N} / \mathrm{A} \quad \mathrm{ICE} \quad \mathrm{F} \overline{\mathrm{i}} \mathrm{rmi} \bar{c}$ tes}

12718_7_92_Firmicutes_Clostridia_Clostridiales_Peptostreptococcaceae_Clos

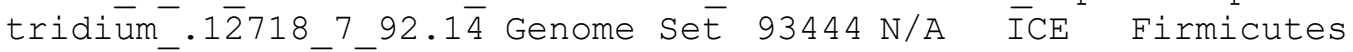

14207_6_3_Bactēroidetes_Bacteroidia_Bacteroidales_Porphyromonadaceae_NA_. $14207^{-6} 3.48$ Genome Set 12094 N/A ICE Bacteroidetes

UMGS41__SRR5275454_bin.24_Firmicutes_Clostridia_Clostridiales_Ruminococcac eae_Anāeromassilib̄acillus__NODE_936_İength_17260_cov_35.7004 - Genome Set $17260 \mathrm{~N} / \mathrm{A} \quad$ ICE Firmicutes

UMGS341_ERR688549_bin.7_Firmicutes_Clostridia_Clostridiales_Peptostreptoc

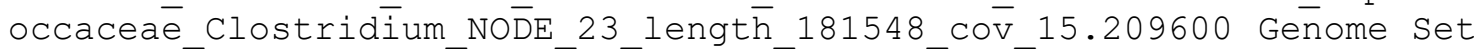
$18 \overline{1548}$
$\mathrm{N} / \mathrm{A}^{-} \quad \mathrm{ICE} \quad \overline{\mathrm{F}}$ irmicutes

NZ_LN849007.1_Clostridium_sp._mt5_genome_assembly_Clostridium_niameyense_ _scaffold_scaffold00006_wholè genome_shotgun_sequence Genome Set
2380284
N/A ICE Firmicutes

21673_4_19_Firmicutes_Clostridia_Clostridiales_Lachnospiraceae_Blautia_.2 $1673 \overline{4} \overline{1} 9 . \overline{2} 5$ Genome set $332 \overline{98} \mathrm{~N} / \mathrm{A}$ ICE Firmicutes

UMGS $\overline{8} 7 \overline{3}$ ERR1190965_bin.23_Firmicutes_Clostridia_Clostridiales_Lachnospira

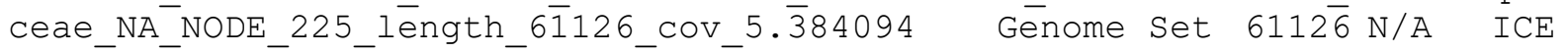
Fírmicutes

NZ_EQ973650.1_Bacteroides_coprophilus_DSM_18228_=_JCM_13818_genomiC_scaff old̄_Scfld22_whole_genome_shotgun_sequence Genome Set $36 \overline{1} 429$ - N/A ICE $\overline{\text { Bacteroidetes }}$

UMGS1689_ERR414250_bin.15_Firmicutes_Clostridia_Clostridiales_Peptostrept

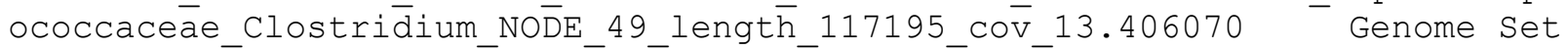
$1171 \overline{95}$
N/A N/A Firmicutes

14207_7_37_Firmicutes_Clostridia_Clostridiales_Lachnospiraceae_Dorea_.142 07 7_37.5 - Genome Set 175725 - N/A ICE Firmicutes

8080_1_93_Firmicutes_Clostridia_Clostridiales_Lachnospiraceae_NA_ERS13819 $1.80 \overline{8} 0^{-}{ }^{1} \overline{9} 3.3$ Genome Set $31 \overline{8391}$ N/A ICE Firmicutes NZ_KQ2 $\overline{3} 5 \overline{8} 47.1$ [Clostridium]_bolteae_WAL-

14578_genomic_scaffold_supercont1.3_whole_genome_shotgun_sequence Genome Set $66822 \overline{4} \quad$ N/A ICE Firmicutes

UMGS367_SRR1197541_bin.30_Tenericutes_Mollicutes_Erysipelotrichales_Erysi pelotrichaceae_Solōbacterīum_NODE_34_Iength_12914̄6_cov_5.963460

Genome sēt $129146 \quad \bar{N} / \mathrm{A}-\overline{I C E}$ Tenéricutes

12718_7_84_Firmicutes_Clostridia_Clostridiales_Ruminococcaceae_NA_.12718_ 7 84. $\overline{6}-$ - Genome Set 272283 - N/A ICE Firmicutes

200287_6_22_Firmicutes_Clostridia_Clostridiales_Ruminococcaceae_Ruminococc

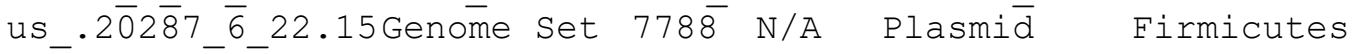

UMGGS286_SRRT2155376_bin.6_Firmicutes_Clostridia_Clostridiales_Ruminococcac

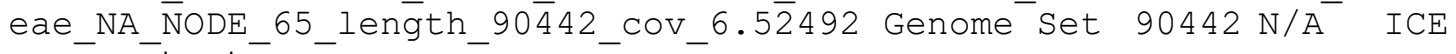

Firmicutes

NZ_LN865152.1_Eubacterium_sp._SB2_genome_assembly_Eubacterium_massiliense scaffold_scaffold00008_whole_genome_shotgun_sequence Genome set
$1069 \overline{3} 80$
N/A I $\overline{C E}$ Firmicutes

UMGS835_ERR589530_bin.45_Bacteroidetes_Bacteroidia_Bacteroidales_Porphyro monadacēae_NA_NODE_432_lēength_44817_cov̄_7.826706-Genome set $4 \overline{4} 817$ N/A ICE Bacteroidetes

UMGS680_ERR525961_bin.2_Firmicutes_Clostridia_Clostridiales_NA_NA_NODE_11

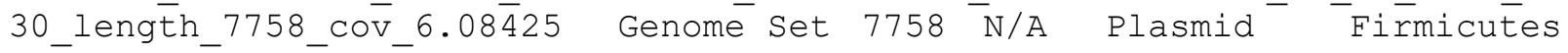


20298249 Firmicutes Bacilli Lactobacillales Streptococcaceae Streptococ cus_. $20 \overline{2} 98$ 2_49.3Genome set 398961 rep_cluster_1663 ICE Firmicutes NZ $\bar{A} C F Y 01000 \overline{1} 13.1$ Roseburia inulinivorans DSM $16841^{-} \mathrm{R}$ inulinivorans1.0.1_Cont472.1 whole_genome_shotgun_sequence $\bar{e}$ Genome set 69762 N/A ICE Firmicutes

14207_7_28_Firmicutes_Clostridia_Clostridiales_NA_NA_.14207_7_28.3 Genome set $7396 \overline{3} 9 \quad$ N/A ICE Firmicutes

UMGS1175_ERR414243_bin.25_Firmicutes_Clostridia_Clostridiales_NA_NA_NODE_

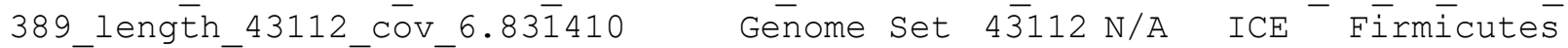
NZ_ĒQ97365ī.1_Bacterōides_coprophilus_DSM_18228_=_JCM_13818_genomic_scaff old__Scfld23_whole_genome_shotgun_sequence Genome Set $20 \overline{5} 697$ N/A ICE Bacteroidetes

UMGS637_SRR5275454_bin.27_Firmicutes_Clostridia_Clostridiales_Ruminococca ceae_NA_NODE_275_lēength_51607_cov_12.2962 Genome set $5160 \overline{7}$ N/A ICE Firmicutes

NZ_JH636044.1_Bacteroides_sp._3_2_5_genomic_scaffold_supercont2.2_whole_ geñome shotgun sequence Geñome set $325 \overline{3} 542 \quad$ N/A ICE

Bacteroidetes

UMGS856_SRR1196456_bin.38_Firmicutes_Clostridia_Clostridiales_Ruminococca

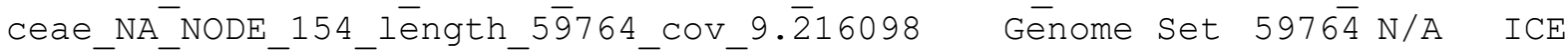
Firmicūtes

NC_010376.1_Finegoldia_magna_ATCC_29328_DNA_complete_genome Genome Set $17975 \overline{77}$ rep_cluster_1663 ICE Firmicutes

20298_3_37_Proteobacteria_Gammaproteobacteria_Enterobacteriales_Enterobac

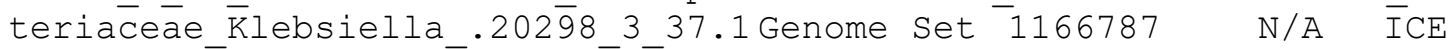

Proteobacteria

UMGS1441_ERR688506 bin.6_Firmicutes_Clostridia_Clostridiales_Eubacteriace

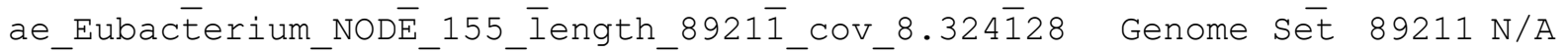

ICE Firmicutes

13414_6_20_Firmicutes_Clostridia_Clostridiales_Lachnospiraceae_Blautia_.1 $3414 \_\overline{6} \_\overline{2} 0 . \overline{3}$ Genome Set $296997-$ N/A ICE Firmicutes

NZ_A $\bar{B} J \bar{L} 02000006.1$ Bacteroides_intestinalis_DSM_17393_B_intestinalis-

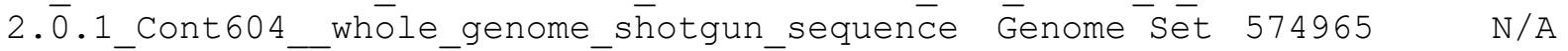
ICE Bacteroidetes

UMGS185_ERR1912997bin.34_Firmicutes_Clostridia_Clostridiales_Ruminococca

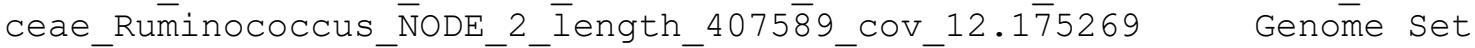

$$
407589 \quad \bar{N} / A \quad \text { ICE Firmicutes }
$$

UMGS1430_ERR1305892_bin.72 Bacteroidetes Bacteroidia Bacteroidales_Prevot

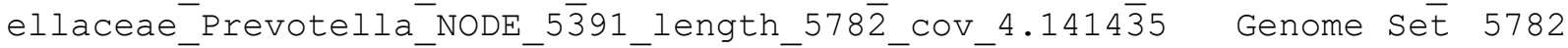
N/A Plasmid ${ }^{-}$Bācterōidetes

NZ_ACFY01000094.1_Roseburia_inulinivorans_DSM_16841_R_inulinivorans-

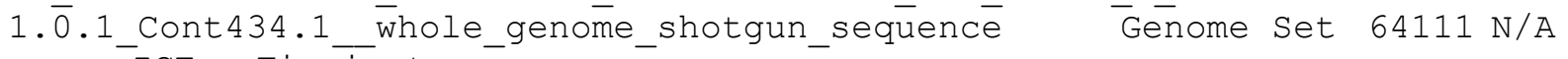
ICE Firmicutes

NZ_GG657974.1_Fusobacterium_sp._3_1_5R_genomic_scaffold_supercont1.4_who le_genome_shotgun_sequence ${ }^{-}$Genome Set $^{-} 173616^{-}$N/A $^{-}$ICE

Fusobacteria

NZ_LN869525.1_Intestinimonas_sp._GD2_genome_assembly_Intestinimonas_massi liensis_scaffold_scaffold00003_whole_genome_shotgun_sequence Genome set $6 \overline{430}$ N/A Plasmid Firmicutes

UMGS798_SRR5275432_bin.24_Firmicutes_Clostridia_Clostridiales_Ruminococca ceae_NA_NODE_706_lēength_18987_Cov_14.977763 Genome set $1898 \overline{7}$ N/A ICE Firmicutes

12718_7_51_Firmicutes_Clostridia_Clostridiales_Lachnospiraceae_Ruminococc

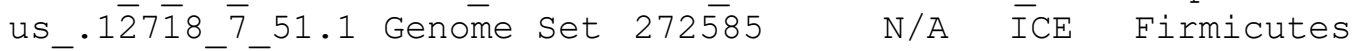


20298_2_23_Bacteroidetes_Bacteroidia_Bacteroidales_Bacteroidaceae_Bactero ides_.2029

12718_7_94_Firmicutes_Clostridia_Clostridiales_Lachnospiraceae_NA_.12718_ $794 . \overline{1} 0^{-}$Genome Set $90751 \mathrm{~N} / \mathrm{A}^{-}$ICE Firmicütes

NZ_DS990163.1_Ruminococcus_lactaris_ATCC_29176_Scfld_02_0_genomic_scaffol d_whole_genome_shotgun_sequence Genome set $172790^{-}-\overline{\mathrm{N}} / \mathrm{A}$ ICE

Firmicutes

13414_6_44_Firmicutes_Clostridia_Clostridiales_Lachnospiraceae_Lachnobact erium .1̄3414 $644.14^{-}$Genome Set $77280 \mathrm{~N} / \mathrm{A}$ ICE Firmicutes

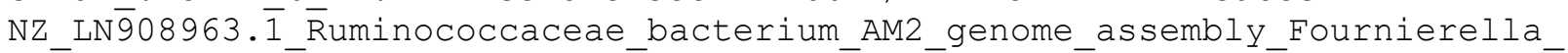
massiliensis_scaffold_scaffoldd00018_whole_genome_shotgun_sequence

Genome Set $52764 \overline{7} \quad$ N/A ICE Firmicutes

NZ_JALA01000016.1_Providencia_alcalifaciens_PAL-

1_čctgN52L138138C40.3604_wholē_genome_shotgün_sequence Genome Set 138138 rep_cluster_1220,rep_cluster_1220 Plasmid

Proteobacteria

UMGS1325_ERR1190866_bin.23 Firmicutes_Clostridia_Clostridiales_Oscillospi raceae_Oscillibacter $\bar{r}$ NODE_T194_length_3488_cov_4.795805 Genome Set 3488
$\overline{\mathrm{N}} / \mathrm{A} \quad \mathrm{N} / \mathrm{A} \quad$ Firmicutes

13414_6_68_Firmicutes_Clostridia_Clostridiales_Lachnospiraceae_NA_.13414 $668 . \overline{6}-$ Genome set 245950 N/A ICE Firmicutes

UMMGS1726_SRR5127498_bin.5_Actinobacteria_Actinobacteria_Coriobacteriales_ Coriobact̄eriaceae_Cōllinsēlla_NODE_199_lēngth_29094_cov_9.795344

Genome set $\overline{2} 9094 \mathrm{~N} / \mathrm{A}$ ICE $\bar{A} c t i \bar{n} o b a c t e \overline{r i a}$

NZ HE610717.1 Peptoniphilus senegalensis JC140 type strain JC140T genom ic_scaffold_scaffold00001_whole_genome_shotgun_sequenceGeñome set

$881198 \quad$ N/A ICE Firmicutes

21673_4_18_Firmicutes_Clostridia_Clostridiales_NA_NA_.21673_4_18.11

Genome Set $1164 \overline{6} 6 \quad$ N/A ICE Firmicutes

NZ_GG705131.1_Acinetobacter_radioresistens_SH164_genomic_scaffold_superco

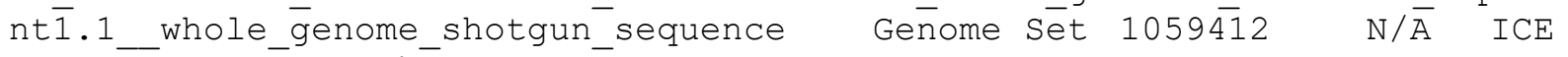

Proteobacteria

14207_7_83_Firmicutes_Clostridia_Clostridiales_Lachnospiraceae_Roseburia_ $.1420 \overline{7} \overline{7} 8 \overline{3} .14$ Genome Set $126 \overline{90} 2$ N/A $\overline{\text { ICE Firmicutes }}$

UMGS670̄ËRR1190964_bin.38_Firmicutes_Clostridia_Clostridiales_NA_NA_NODE

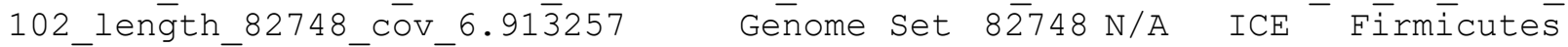

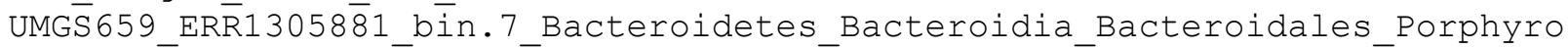
monadaceae_NA_NODE_225_length_98141_cov_8.194187 Genome set $9 \overline{8} 141$ N/A

ICE Bacteroidetes

UMGS1537_ERR1912950_bin.18_Firmicutes_Clostridia_Clostridiales_Peptostrep

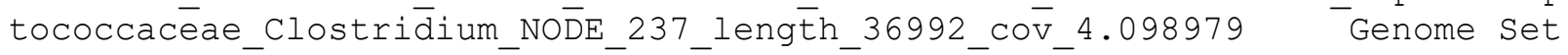
$36992 \overline{\mathrm{N}} / \mathrm{A} \quad \mathrm{ICE} \quad \overline{\mathrm{F}} \mathrm{irmi} \overline{\mathrm{C}} \mathrm{utes}$

18048_1_69_Firmicutes_Clostridia_Clostridiales_Lachnospiraceae_NA_.18048_ 1_69.13- Genome Set 80451 N/A ICE Firmicutes

144207_7_37_Firmicutes_Clostridia_Clostridiales_Lachnospiraceae_Dorea_.142 $077 \overline{3} 7.9-$ Genome Set $129942-$ N/A ICE Firmicutes

$20 \overline{2} 8 \overline{7}$ 6_22_Firmicutes_Clostridia_Clostridiales_Ruminococcaceae_Ruminococc us_.20287 $\overline{6} \_22.3$ Genome Set $424 \overline{267}$ N/A ICE Firmicutes

NZ_DS264271.1_Eubacterium_ventriosum_ATCC_27560_Scfld029_genomic_scaffold whole_genomé_shotgun_sequence Genome Set $2 \overline{9} 1583$ N/A I $\bar{C} E$ Firmicutes

UMGS781_ERR1305892_bin.56_Proteobacteria_Deltaproteobacteria_Desulfovibri

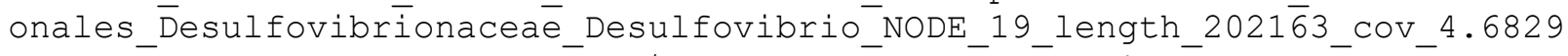
62 Genome set $202163-$ N/A ICE $\overline{\text { Proteōbacteria }}$ 
13414_6_68_Firmicutes_Clostridia_Clostridiales_Lachnospiraceae_NA_.13414_ 6_68.19- Genome Set 28274 N/A N/A Firmicutes

147207_7_89_Firmicutes_Clostridia_Clostridiales_NA_NA_.14207_7_89.45

Genome Set $2587 \overline{7}$ N/A N/A Firmicutes

UMGS1329_ERR1018298_bin.48 Firmicutes_Clostridia_Clostridiales_Lachnospir

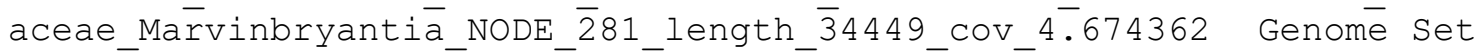
$34449 \mathrm{~N} / \mathrm{A} \quad \mathrm{N} / \mathrm{A}^{-}$Firmicutes

UMGS1158_DRR042394_bin.56_Firmicutes_Clostridia_Clostridiales_Lachnospira

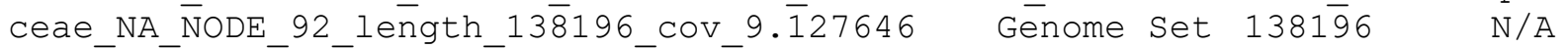
I $\overline{C E} \quad \overline{\text { Firmicutes }}$

UMGS62_SRR492197_bin.1_Firmicutes_Clostridia_Clostridiales_Clostridiales Family_XI_Peptonīphilus__NODE_7_lēngth_311400_COV_89.688776 ${ }^{-}$Genome Set $\overline{3} 1 \overline{4} 00 \quad$ N/A İE Firmicutes

12718_7_47_Firmicutes_Clostridia_Clostridiales_NA_NA_.12718_7_47.7 Genome set $2445 \overline{3} 1 \quad$ N/A ICE Firmicutes

UMGS1754_ERR505083_bin.47_Firmicutes_Clostridia_Clostridiales_Peptostrept

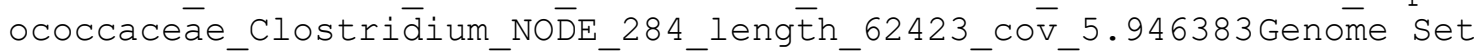

$6242 \overline{3} \mathrm{~N} / \mathrm{A} \quad \mathrm{ICE}$ Firmicutēs

14207_7_83_Firmicutes_Clostridia_Clostridiales_Lachnospiraceae_Roseburia_ $.1420 \overline{7} \overline{7}+8 \overline{3} .19$ Genome set $921 \overline{89}$ N/A ICE Firmicutes UMGS1684_ERR011192_bin.15_Bacteroidetes_Bacteroidia_Bacteroidales_Prevote llaceae_Prevotella_NODE_1_length_224553_Cov_24.0209Genome Set $22 \overline{4} 553$

$\mathrm{N} / \mathrm{A} \quad \mathrm{ICE}$ Bacteroidetes

UMGS488_SRR5275456_bin.21_Firmicutes_Clostridia_Clostridiales_Lachnospira ceae_Dorea_NODE_30 $\overline{6}$ length_66612_cov_40.2722 Genome set $6661 \overline{2}$ N/A ICE

Firmicutes

UMGS901 SRR6028340 bin.15 Bacteroidetes Bacteroidia Bacteroidales Bactero

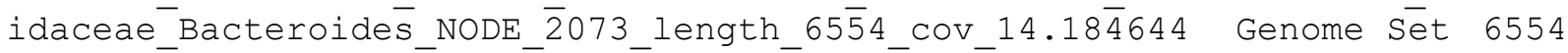
N/A Plasmid Bäcterōidetes

UMGS2_ERR1190731_bin.19_Bacteroidetes_Bacteroidia_Bacteroidales_Porphyrom

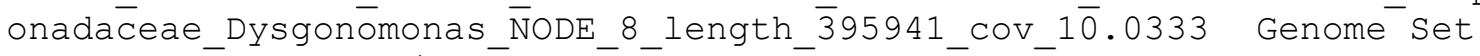
$395 \overline{9} 41$ N/A ICE Bacteroidetes

14207_7_59_Firmicutes_Clostridia_Clostridiales_NA_NA_.14207_7_59.1 Genome set $1062 \overline{6} 44 \quad$ N/A ICE Firmicutes

18048 2_67_Firmicutes_Clostridia_Clostridiales_Lachnospiraceae_Blautia_.1 $8048 \overline{2} \overline{6} 7 . \overline{2} 2$ Genome set $789 \overline{2}$ N/A ICE Firmicutes

UMGS935_SRR5050584_bin.5_Firmicutes_Clostridia_Clostridiales_Lachnospirac

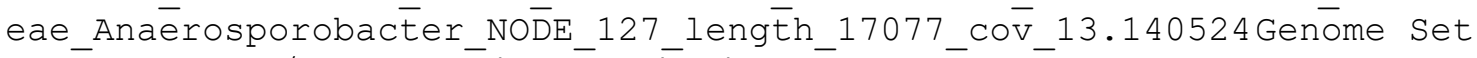
$17077 \mathrm{~N} / \mathrm{A}$ Plasmid Firmicutes

8080_1_93_Firmicutes_Clostridia_Clostridiales_Lachnospiraceae_NA_ERS13819

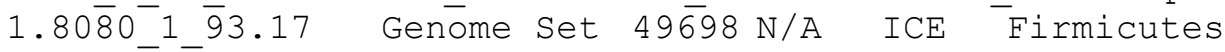
UMGS533_ERR1293593_bin.31_Firmicutes_Clostridia_Clostridiales_Ruminococca

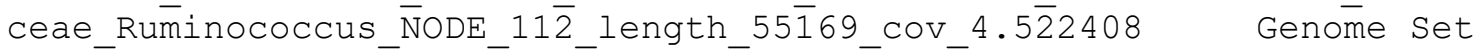
$55169 \mathrm{~N} / \mathrm{A} \quad \overline{\mathrm{N}} / \mathrm{A} \quad$ Firmicutes

NZ_GG703855.1_Prevotella_copri_DSM_18205_genomic_scaffold_Scfld3_whole_g enome_shotgun_sequence Gēnome Set $\overline{2} 94362^{-}$N/A ICE Bacterōidetes NZ_DS264281.1_Eubacterium_ventriosum_ATCC_27560_Scfld0219_genomic_scaffol d_whole_genome_shotgun_sēquence Geñome Set $6 \overline{2} 353$ N/A ICE Firmicutes UMGGS98SRRR1761707_bin.48 Eirmicutes_Clostridia_Clostridiales_Ruminococcac eae_NA_NODE_492_lēength_27138_COV_4.013883 Genome set $271 \overline{3} 8$ N/A ICE $\overline{\text { Firmicutes }}$

11861_6_58_Firmicutes_Clostridia_Clostridiales_Lachnospiraceae_Blautia_.1 1861_ $\overline{6} \_\overline{5} 8 . \overline{2}$ Genome Set $447767-$ N/A ICE Firmicutes 
UMGS1111_ERR414291_bin.37_Bacteroidetes_Bacteroidia_Bacteroidales_Porphyr omonadacēae_NA_NODE_1318_īength_19672_Cov_4.305704 Genome set $19 \overline{6} 72$ N/A ICE Bacteroidetes

20287_6_9_Bacteroidetes_Bacteroidia_Bacteroidales_Bacteroidaceae_Bacteroi des_. $\overline{2} 0 \overline{2} 8 \overline{7} 6$ 6.8 Genome ${ }^{-}$Set $294307^{-}$N/A ICE ${ }^{-}$Bacteroidetes

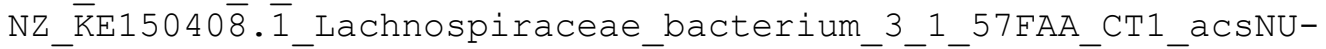

supercont1.4_whole_genome_shotgun_sequence Genome Set 825043 N/A

ICE Firmicutes

UMGS1028_SRR341596_bin.15_Tenericutes_Mollicutes_Erysipelotrichales_Erysi

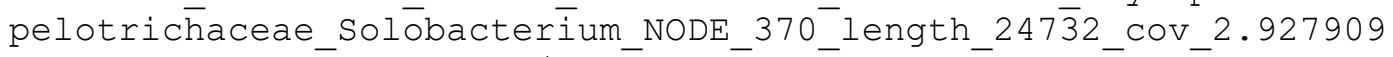

Genome Set 24732 N/A ICE Tenericutes

UMGS777_ERR1305885_bin.21_Firmicutes_Clostridia_Clostridiales_NA_NA_NODE_

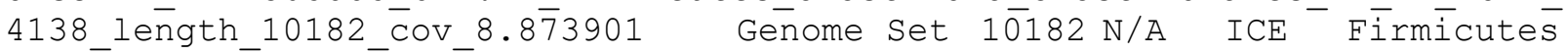

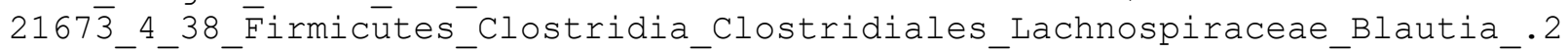
1673 4 $38 . \overline{1}$ Genome Set 452966 N/A ICE Firmicutes

8080_1_77_Firmicutes_Clostridia_Clostridiales_Peptostreptococcaceae_Clost

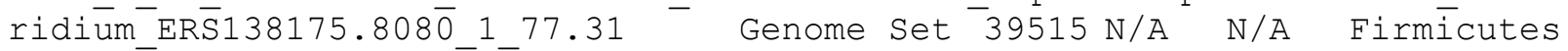
NZ_GL53̄8231.1_Faecalib̄acterium_cf._prausnitzii_KLE1255_genomic_scaffold_S cfīd25_whole_genome_shotgun_séquence Genome- Set 5889 N/A N/A

\section{Firmicutes}

UMGS491_SRR5275479_bin.22_Firmicutes_Clostridia_Clostridiales_NA_NA_NODE

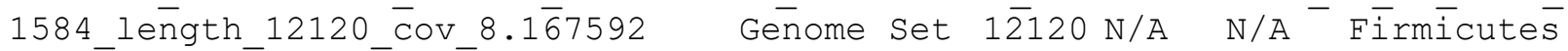
20298_3_58_Ēirmicutes_Clostridia_Clostridiales_NA_NA_.20298_3_58.3

Genome set $3133 \overline{3} 1 \quad$ N/A ICE Firmicutes

UMGS781 ERR1305892 bin.56 Proteobacteria Deltaproteobacteria Desulfovibri

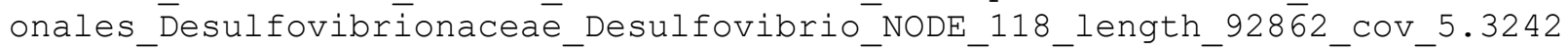
00 Genome set $92862 \mathrm{~N} / \mathrm{A}^{-}$ICE Proteōbactēria

21673 4_71_Firmicutes_Clostridia_Clostridiales_Peptostreptococcaceae_clos

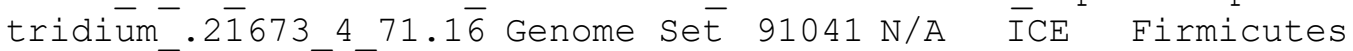

UMGS207_SRR3917 $\overline{8} \overline{8} 6$ bin.8_Firmicutes_Clostridia_Clostridiales_NA_NA_NODE_5 059 length_2353_cov $6.60 \overline{8} 79$ Genome Set 2353 N/A ICE Firmicutes

NZ_ĀBXA01000002.1_Añaerococcus_hydrogenalis_DSM_7454_A_hydrogenalis-

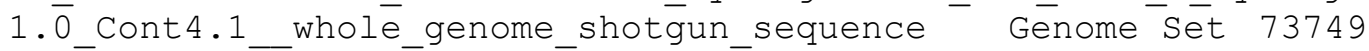

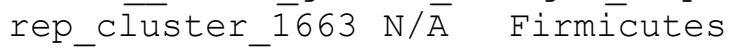

UMGS1488_SRR5057076_bin.64_Firmicutes_Clostridia_Clostridiales_Eubacteria

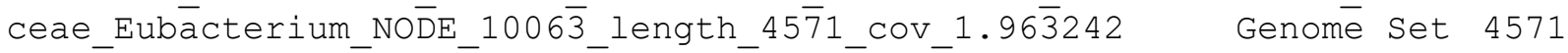

$$
\text { N/A ICE Firmícutes }
$$

14207_6_3_Bacteroidetes_Bacteroidia_Bacteroidales_Porphyromonadaceae_NA_. 14207_6_3.24 Genome Set 39133 N/A ICE Bacteroidetes

UMGS941_ERR1190721_bin.6_Proteobacteria_Betaproteobacteria_Burkholderiale s_Sutterellaceae_Sutterēlla_NODE_1586_lèngth_10652_cov_3.78239 Genome Set $10652 \mathrm{~N} / \mathrm{A}$ ICE Protēobacteria

NZ_KI270975.1_Faecalitalea_Cylindroides_ATCC_27803_Scaffold17_whole_geno me_shotgun_sequence Genome set 52396 N/A Plasmid Tenericutes 21673_4_38_Firmicutes_Clostridia_Clostridiales_Lachnospiraceae_Blautia_.2 $1673 \overline{4} \overline{3} 8 . \overline{8}$ Genome Set $164669-$ N/A ICE Firmicutes

$2042 \overline{7}$ 4_25_Firmicutes_Clostridia_Clostridiales_Lachnospiraceae_Lachnobact erium_. $204 \overline{2} 7$ 4_25.161- Genome Set 392 N/A P̄lasmid Firmicutes 20298_3_57_Actinobacteria_Actinobacteria_Coriobacteriales_Coriobacteriace ae NA_.'.20298_3_57.8 Geñome Set 149029 N/A ICE Àctinobacteria 14207_7_56_Firmicutes_Clostridia_Clostridiales_NA_NA_.14207_7_56.16 Genome set $9133 \overline{8}$ N/A ICE ${ }^{-}$Firmicutes 
8080_1_80_Tenericutes_Mollicutes_Erysipelotrichales_Erysipelotrichaceae_S olobācterium_ERS138178.8080_1_80.7Genome set $14704 \overline{2}$ N/A ICE

Tenericutes

12718_7_84_Firmicutes_Clostridia_Clostridiales_Ruminococcaceae_NA_.12718_ $784 . \overline{2}-$ Genome Set 497218 - N/A ICE Firmicutes

20287_6_6_Proteobacteria_Gammaproteobacteria_Enterobacteriales_Enterobact

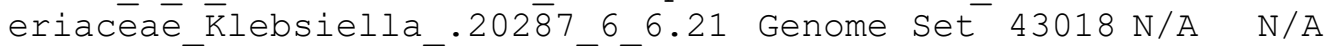

Proteobacteria

UMGS119_ERR1620292_bin.17_Firmicutes_Clostridia_Clostridiales_Lachnospira ceae_Blāutia_NODE_80_lengt̄h_99411_cov_z23.671716 ${ }^{-}$Genome Set 99411 N/A ICE Firmicutes

NZ_KI392040.1_Helicobacter_bilis_ATCC_43879_genomic_scaffold_supercont2.9 whole_genomé_shotgun_sequence ${ }^{-}$Genome Set $101470 \overline{4}$ N/A ${ }^{-}$ICE Proteobacteria

UMGS698 ERR414252 bin.16 Bacteroidetes Bacteroidia Bacteroidales Bacteroi daceae Bacteroides NODE $\overline{6} 9$ length $1212 \overline{7} 7 \mathrm{cov} 35.26 \overline{2} 263$ Genome $\overline{\text { Set }}$
121277
N/A ICE Bacteroidetes

NZ_GL384001.1_Burkholderiales_bacterium_1_1_47_genomic_scaffold_supercont

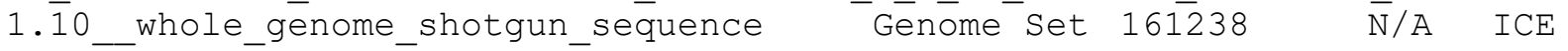
Proteobactería

13470_2_92_Firmicutes_Clostridia_Clostridiales_Lachnospiraceae_NA_.13470_ $292 . \overline{4} 1^{-}$Genome Set $34990 \mathrm{~N} / \mathrm{A}^{-}$ICE Firmicütes

NZ̄_JALB01000021.1_Providencia_alcalifaciens_RIMD_1656011_ctgN83L106367C26 $.6 \overline{9} 83$ _whole_genome_shotgun_sequence Genome set 106367

rep cluster 1220, rep cluster 1220 N/A Proteobacteria

UMGS1768_SRR5275462_bin.23_Bacteroidetes_Bacteroidia_Bacteroidales_NA_NA_

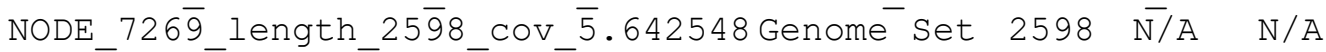

Bacteroidetes

UMGS1234_SRR3582140_bin.13_Firmicutes_Clostridia_Clostridiales_NA_NA_NODE

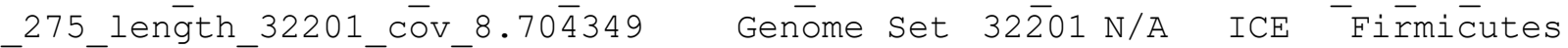

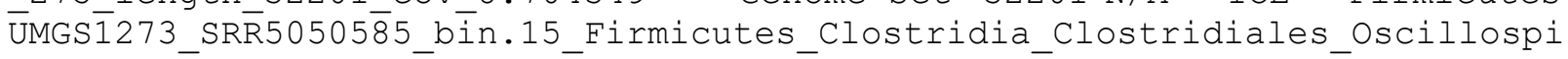
raceae_Oscillibacter_NODE_2719_length_4572_Cov_5.194598 Genome set 4572

$\overline{\mathrm{N}} / \mathrm{A} \quad \mathrm{N} / \mathrm{A} \quad$ Firmicutēs

UMGS1179 ERR1293813 bin.25_Bacteroidetes_Bacteroidia_Bacteroidales_NA_NA_ NODE_691_length_2088̄9_Cov_3.246040Genome Set 20889 N/A ICE

Bacteroidetes

NZ_FMIX01000004.1_Streptococcus_sp._Marseille-

P29̄15_whole_genome_shotgun_sequence Genome set 1305823 N/A ICE Firmicutes

13414_6_41_Firmicutes_Clostridia_Clostridiales_Lachnospiraceae_Blautia_.1 $3414 \overline{6} \overline{4} 1 . \overline{2}$ Genome Set 610726 - N/A ICE Firmicutes

UMGS $\overline{6} 9 \overline{2}$ ERR1913113 bin.1 Firmicutes Clostridia Clostridiales NA NA NODE 9 length_124713_cov_10.934̄212 Genome Set $124713^{-}$N/A ICE ${ }^{-} \overline{\text { Firmicutes }}$ 12718_7_47_Firmicutes_Clostridia_Clostridiales_NA_NA_.12718_7_47.8 Genome set $2229 \overline{9} 7 \quad$ N/A ICE Firmicutes

UMGS1735_ERR525802 bin.17 Firmicutes Clostridia_Clostridiales Ruminococca ceae_NA_T̄NODE_631_lēength_19̄458_cov_9.56326 Genome set $1945 \overline{8}$ N/A N/A Firmicutes

UMGS617 ERR011209 bin.1 Bacteroidetes Bacteroidia Bacteroidales Prevotell

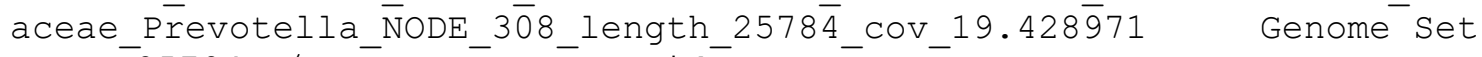
-25784 N/A ICE Bacteroidētes

UMGS1917_ERR1190590_bin.14_Firmicutes_Clostridia_Clostridiales_Peptostrep

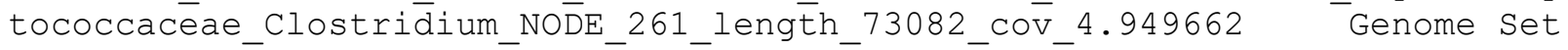
$73082 \overline{\mathrm{N}} / \mathrm{A} \quad \mathrm{N} / \mathrm{A} \quad \overline{\mathrm{F}} \mathrm{irmi} \bar{c} u t e \bar{s}$ 
14207_7_1_Firmicutes_Clostridia_Clostridiales_Lachnospiraceae_Lachnospira $.142 \overline{0} \overline{7}^{-}{ }^{-} 1.1$ Genome Set $55 \overline{7} 787$ N/A ICE Firmicutes

UMMGS 422-SRRR5558166 bin.58 Bacteroidetes Bacteroidia Bacteroidales Bactero idaceae Bacteroide $\bar{s}$ NODE $\overline{1} 374$ length $24 \overline{4} 38$ cov $6.16 \overline{3} 910$ Genome Set $2 \overline{4} 438 \mathrm{~N} / \mathrm{A} \quad \mathrm{ICE} \quad$ Bäcterōidetes

UMGS618_SRR4408228_bin.21_Firmicutes_Clostridia_Clostridiales_NA_NA_NODE_ 6320 leñgth $2264 \mathrm{COV} 38.9 \overline{3} 53$ Genome Set 2264 N/A Plasmid F $\overline{\text { Armicutes }}$ UMGS $\overline{6} 30$ ERR'̄ $6203 \overline{2} 0$ bìn.42 Bacteroidetes Bacteroidia Bacteroidales Bactero

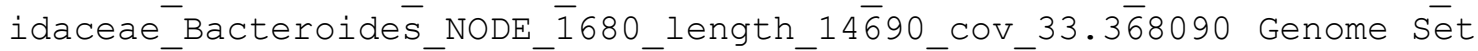

$1 \overline{4} 690 \mathrm{~N} / \mathrm{A}$ ICE Bäterōidetes

NZ_CYUJ01000003.1_Neofamilia_massiliensis_strain_SIT13_whole_genome_shot

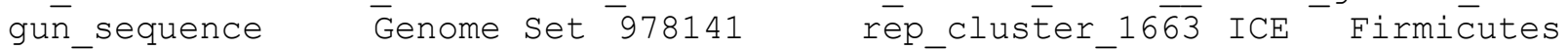
NZ_FMIY01000002.1_Veillonellaceae_bacterium_MarseilieP2 $\overline{9} 11$ _whole_genome_shotgun_sequeñce Genōme set 1611777 N/A ICE Firmicutes

14207_7_37_Firmicutes_Clostridia_Clostridiales_Lachnospiraceae_Dorea_.142 07 7 $37.16^{-}$Genome Set 86428 N/A ${ }^{-}$ICE Firmicutes

UMGGST1134_ERR1620298_bin.15_Firmicutes_Clostridia_Clostridiales_Lachnospir aceae_NA_NODE_9_length_231593_cov_16.395175 Geñome set $23159 \overline{3}$ N/A

\section{ICE Firmicutes}

NZ_KE161031.1_Cedecea_davisae_DSM_4568_Scaffold1_whole_genome_shotgun_se quēnce Genome set 9581 rep_clustēr_1155 plasmid Proteōbacteriā 12718_7_51_Firmicutes_Clostridia_Clostridiales_Lachnospiraceae_Ruminococc

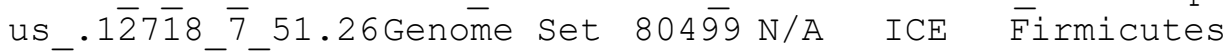

UMGGS1158_D̄RR 042394 bin.56_Firmicutes_Clostridia_Clostridiales_Lachnospira ceae_NA_N̄ODE_839_lēength_22246_cov_7. 173335 Genome set $2224 \overline{6}$ N/A ICE

Firmicutes

UMGS2007_ERR209576_bin.9_Bacteroidetes_Bacteroidia_Bacteroidales_Bacteroi daceae_Bäcteroides_NODE_2787_length_2712_cov_2.8389̄16 Genome Set 2712

$\overline{\mathrm{N}} / \mathrm{A} \quad \mathrm{N} / \mathrm{A} \quad \overline{\mathrm{Ba}} \mathrm{ctero} i \mathrm{det} \overline{\mathrm{e}} \mathrm{s}$

UMGS200_SRR6028624_bin.16_Firmicutes_Clostridia_Clostridiales_Ruminococca

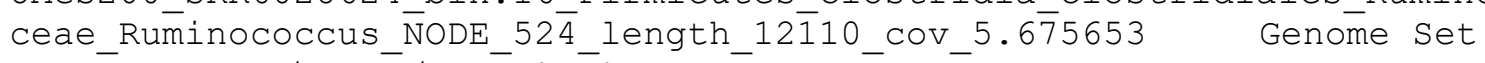
$12110 \mathrm{~N} / \mathrm{A} \quad \overline{\mathrm{N}} / \mathrm{A} \quad$ Firmicutes

20287_6_63_Firmicutes_Clostridia_Clostridiales_Lachnospiraceae_NA_.20287_ 6_63.1. - Genome Set 749211 N/A ICE Firmicutes

UM̄GS1672_ERR2013583_bin.9_Firmicutes_Clostridia_Clostridiales_Peptostrept

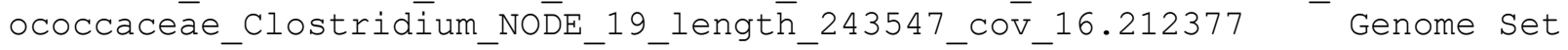
$2435 \overline{4} 7$ N/A ICE Firmicutes

20287 6_9 Bacteroidetes_Bacteroidia_Bacteroidales_Bacteroidaceae_Bacteroi des_. $20 \overline{2} 8 \overline{7}$ 6_9.1 Genome Set $103887 \overline{0}^{-}$N/A ICE ${ }^{-}$Bacteroidetes 13470_2_67_Bäcteroidetes_Bacteroidia_Bacteroidales_Porphyromonadaceae_Par abactēeroidēs_.13470_2_67.13 Genome Set 32798 rep_cluster_1702 N/A Bacteroidetes

UMGS278_ERR1190624_bin.64_Bacteroidetes_Bacteroidia_Bacteroidales_Prevote llaceae_Prevotella_NODE_50_length_161053_cov_36.628 $\overline{4} 92$ Genome Set $1 \overline{6} 1053 \quad \mathrm{~N} / \mathrm{A} \quad \overline{\mathrm{I}} \mathrm{CE}{ }^{-}$Bacteroidetes

UMGS341_ERR688549_bin.7_Firmicutes_Clostridia_Clostridiales_Peptostreptoc

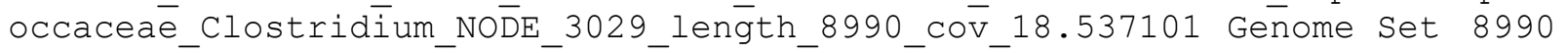
$\mathrm{N} / \overline{\mathrm{A}}$ ICE Firmicutes

20298_3_19_Proteobacteria_Gammaproteobacteria_Enterobacteriales_Enterobac

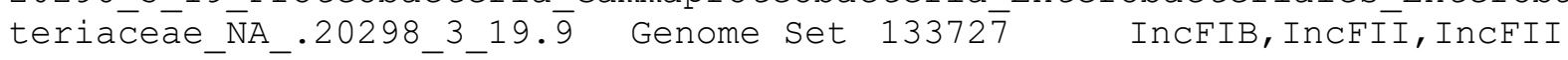
Plasmid Proteobacteria 
UMGS250_SRR2857970_bin.66_Proteobacteria_Deltaproteobacteria_Desulfovibri

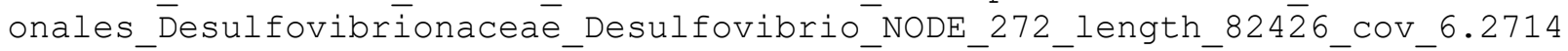
30 Genome set $82426 \mathrm{~N} / \mathrm{A}^{-}$ICE Proteobacteria

UMGS1576 ERR2013621 bin.20 Firmicutes Clostridia Clostridiales Peptostrep tococcacēae_Clostridium_Nō̄E_235_length_68591_cov 6.536010 Genome Set $68591 \overline{\mathrm{N}} / \mathrm{A} \quad \mathrm{N} / \mathrm{A} \quad \overline{\mathrm{F}} \mathrm{irmi} \overline{\mathrm{Cute}} \mathrm{s}$

14207_7_56_Firmicutes_Clostridia_Clostridiales_NA_NA_.14207_7_56.36 Genome set $1212 \overline{8}$ N/A N/A Firmicutes

13414_6_50_Firmicutes_Clostridia_Clostridiales_Lachnospiraceae_Dorea_.134 14 6_50.29 Genome Set 12088 N/A ${ }^{-}$ICE Firmicütes

NZ_DS990135.1_Bacteroides_plebeius_DSM_17135_Scfld_02_18_genomic_scaffold Whole_genomé_shotgun_sequence Genome set $1537 \overline{19}{ }^{-}$- rep_cluster_1702 ICE Bacteroidetes

20287653 Bacteroidetes Bacteroidia Bacteroidales Bacteroidaceae Bactero

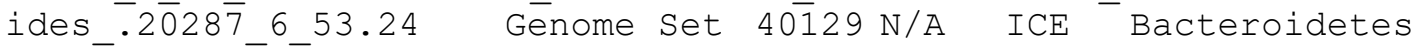

UMGST1972_SRR̄3992959_bin.45_Bacteroidetes_Bacteroidia_Bacteroidales_Prevot

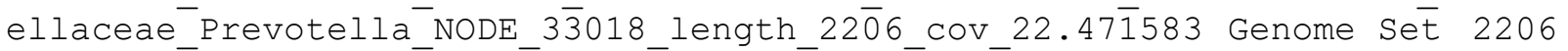

$\mathrm{N} / \overline{\mathrm{A}} \quad \mathrm{ICE}$ Bacteroìdetes

UMGS288_SRR5579983_bin.99_Bacteroidetes_Bacteroidia_Bacteroidales_Bactero

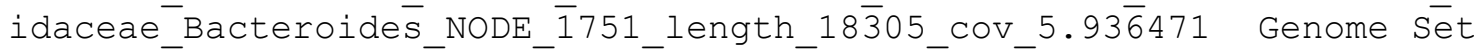

$1 \overline{8} 305 \mathrm{~N} / \mathrm{A} \quad \mathrm{IC} \overline{\mathrm{E}}$ Bācterōidetes

20298_3_63_Bacteroidetes_Bacteroidia_Bacteroidales_Rikenellaceae_Alistipe S_.20298_3_63.4 Genome Set 320309 - N/A ICE Bacteroidetes

UMGGS1826_ERR1305891_bin.67_Firmicutes_Clostridia_Clostridiales_RuminococC

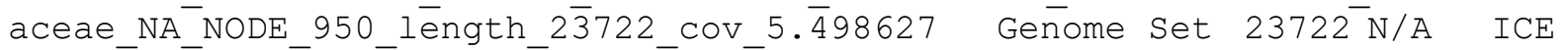

Firmicutes

12718_7_49_Proteobacteria_Gammaproteobacteria_Enterobacteriales_Enterobac teriacēeae_Enterobacter_.127718_7_49.23 Genomē set 69006 IncFII Plasmid Proteobactería

13470_2_65_Bacteroidetes_Bacteroidia_Bacteroidales_Bacteroidaceae_Bactero

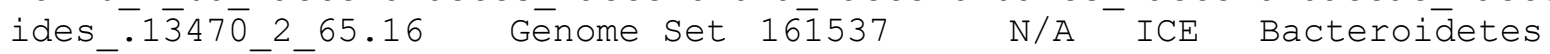
UMGST̈339_SRR $\overline{5} 58100$ bin.10_Actinobacteria_Actinobacteria_Coriobacteriales _Coriobacteriaceae_Collinsēlla_NODE_325_lēength_27077_cov_4.002148

Genome set $2 \overline{7} 077 \mathrm{~N} / \mathrm{A}$ ICE ACtinobactería

UMGS1685 ERR1305900 bin.95 Firmicutes Clostridia Clostridiales NA NA NODE 291_lenḡth_75340_cov_16.0̄65059 Genome set $75 \overline{3} 40$ N/A ICE Firmicutes

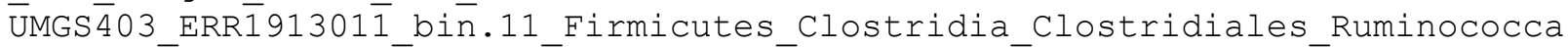
ceae_NA_NODE_153_lēength_48236_Cov_5. 928395 Genome set $4823 \overline{6}$ N/A ICE Firmicutes

14207_7_90_Firmicutes_Clostridia_Clostridiales_NA_NA_.14207_7_90.35 Genome Set $3134 \overline{4}$ N/A N/A Firmicutes

UMGS1416_ERR321433_bin.7_Bacteroidetes_Bacteroidia_Bacteroidales_Bacteroi daceae_Bacteroides_NODE_539_length_12957_Cov_2.881344 Genome Set $\overline{1} 2957 \mathrm{~N} / \mathrm{A} \quad \mathrm{N} / \mathrm{A} \quad \bar{B}$ actèroidetés

UMGS119_ERR1620292_bin.17_Firmicutes_Clostridia_Clostridiales_Lachnospira ceae_Blāutia_NODE_T̄237_leñgth_14870_cov_15.408977 Genome set 14870 N/A ICE Firmicutes

NZ_JH414698.1_Subdoligranulum_sp._4_3_54A2FAA_genomic_scaffold_supercont1 .1_whole_genome_shotgun_sequence Genome set $1096723^{-}$N/A ICE Firmicutes

11861_6_58_Firmicutes_Clostridia_Clostridiales_Lachnospiraceae_Blautia_.1 $1861 \overline{6} \overline{5} 8 . \overline{2} 4$ Genome Set $466 \overline{91} \mathrm{~N} / \mathrm{A}$ ICE Firmicutes

$1341 \overline{4}$ 6_3_Firmicutes_Clostridia_Clostridiales_NA_NA_.13414_6_3.3 Genome set $425 \overline{2} 15 \quad$ N/Ā ICE Firmicutēes 
20298_3_39_Firmicutes_Clostridia_Clostridiales_NA_NA_.20298_3_39.26

Genome set $2975 \overline{5}$ N/A ICE ${ }^{-}$Firmicutes

NZ KE150406.1 Lachnospiraceae bacterium 31 57FAA CT1 acsNU-

supercont1.2_whole_genome_shōtgun_sequence- Genome Set 1273477 N/A

ICE Firmicutes

UMGS 469 SRR5275479 bin.2 Bacteroidetes_Bacteroidia_Bacteroidales_NA_NA_NO DE_58_lēength_98640_Cov_2 9.364680 Genome set $9864 \overline{0}$ N/A N/A

Bacteroidetes

UMGS1968_ERR414319_bin.56_Firmicutes_Clostridia_Clostridiales_Peptostrept

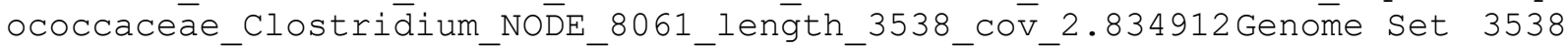

N/A N/A Firmicutes

20287_6_37_Firmicutes_Clostridia_Clostridiales_NA_NA_.20287_6_37.3

Genome set $2093 \overline{8} 7 \quad$ N/A

2028769 Bacteroidetes Bacteroidia Bacteroidales Bacteroidaceae Bacteroi des_. $20 \overline{2} 8 \overline{7} 6$ 6.6 Genome Set $355753^{-}$N/A ICE ${ }^{-}$Bacteroidetes

1420̄76_2_Bacteroidetes_Bacteroidia_Bacteroidales_Porphyromonadaceae_NA_. $14207{ }^{-}{ }^{-}$-66 Genome Set 6092 N/A N/A Bacteroidetes

NZ_GL $88 \overline{2} 606.1$ Bacteroides_fluxus_YIT_12057_genomic_scaffold_Scfld5_whole _genome_shotgün_sequence - Genome set $15 \overline{2} 390 \quad$ N/A ICE

Bacteroidetes

UMGS145_ERR1620270_bin.10_Bacteroidetes_Bacteroidia_Bacteroidales_Prevote

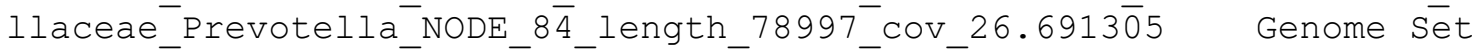

$7 \overline{8997} \mathrm{~N} / \mathrm{A} \quad \overline{\mathrm{C}} \mathrm{E}$ Bactereroidetes

NZ_GG730300.1_Citrobacter_youngae_ATCC_29220_Scfld1_whole_genome_shotgun sēquence Genome Set $81 \overline{4885}$ N/A ICE Proteobactería

UMGS1433_SRR6028647_bin.11_Bacteroidetes_Bacteroidia_Bacteroidales_Prevot

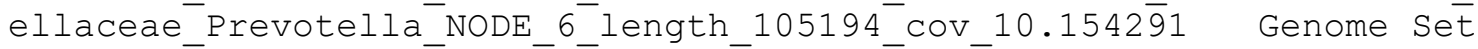

$10 \overline{5194} \mathrm{~N} / \overline{\mathrm{A}} \quad \mathrm{I} \overline{\mathrm{C}} \mathrm{E}$ Bacteroidetes

20298_3_63_Bacteroidetes_Bacteroidia_Bacteroidales_Rikenellaceae_Alistipe

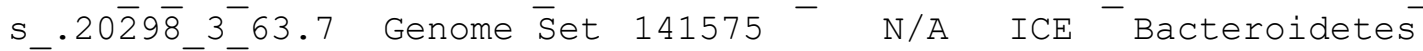

UMGGS491_SRR5275479_bin.22_Firmicutes_Clostridia_Clostridiales_NA_NA_NODE_

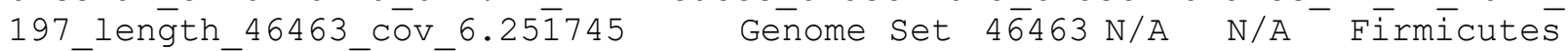

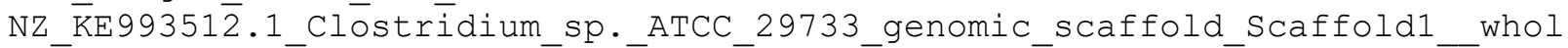
e_genome_shotgun_sequence Genome set 257832 - N/A ICE Firmicutes NZ JH992 $\overline{9} 44.1$ Bacteroides oleiciplenus YIT 12058 genomic scaffold superco

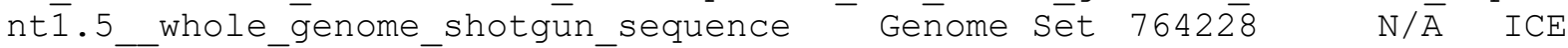

Bacteroidetes

UMGS1064_ERR321174_bin.8_Bacteroidetes_Bacteroidia_Bacteroidales_Porphyro monadaceāe_Parabacteroidés_NODE_4174_lēngth_2456_cōv_3.7651 Gēnome Set

$2456^{-}$N/A N/A Bacteroì̄etes

NZ_FMIZ01000007.1_Ruminococcus_sp._Marseille-

P29̄76_whole_genome_shotgun_sequence Genome set 1698311 N/A ICE Firmicutes

UMGS920_SRR6028474_bin.9_Firmicutes_Clostridia_Clostridiales_Lachnospirac

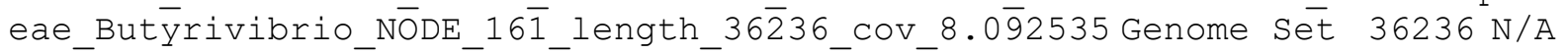
N/A Firmicutes

UMGS1025_ERR1018185_bin.37_Bacteroidetes_Bacteroidia_Bacteroidales_Porphy romonadaceae_NA_NODE_ 22 length_128322_Cov 4.594221 Genome Set $128 \overline{3} 22$

N/A ICE Bacteroidetes

UMGS83_ERR911953_bin.80_Firmicutes_Clostridia_Clostridiales_NA_NA_NODE_23

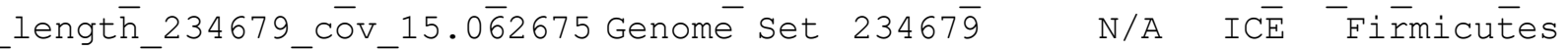
UMMGS920_SRR6028474_bin.9_Firmicutes_Clostridia_Clostridiales_Lachnospirac

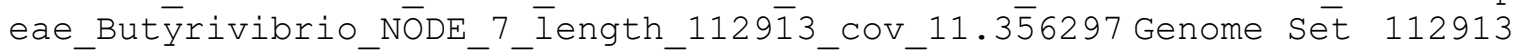

N/A ICE Firmicutes 
21673_4_71_Firmicutes_Clostridia_Clostridiales_Peptostreptococcaceae_Clos

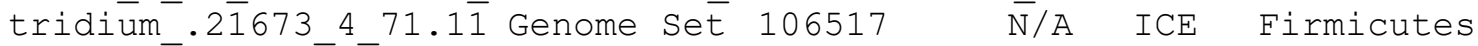
20287_6_37_Firmicutes_Clostridia_Clostridiales_NA_NA_.20287_6_37.24 Genome set $4253 \overline{5}$ N/A ICE ${ }^{-}$Firmicutes

13414_6_2_Firmicutes_Clostridia_Clostridiales_NA_NA_.13414_6_2.7

Genome set $183 \overline{9} 02 \quad \mathrm{~N} / \bar{A}$ ICE Firmicutēs

NZ_GG705174.1_Bacteroides_sp._2_1_22_genomic_scaffold_supercont1.2_whole _genome_shotgü_sequence Genome Set $1170 \overline{0} 23$ N/A ICE

Bacteroidetes

UMGS821_ERR525999_bin.11_Firmicutes_Clostridia_Clostridiales_NA_NA_NODE_1 65 length_69863_cov_6.227581 Genome Set 69863 N/A N/A Firmicutes

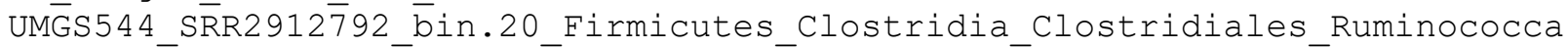
ceae_NA_NODE_350_lēngth_4 $\overline{7} 651$ COV_18.464829 Genome set $4765 \overline{1}$ N/A ICE

Firmicutes

UMGS32_ERR1018211_bin.54_Tenericutes_Mollicutes_NA_NA_NA_NODE_215_length_ $61909 \overline{C O V} 55.4511 \overline{2} 7$ Génome set $61 \overline{9} 09 \mathrm{~N} / \mathrm{A}$ N/A ${ }^{-}$Tenericutes

NZ_CDGTT01000006.1_Mobilicoccus_sp._SIT2_genome_assembly_Mobilicoccus_mass iliensis_SIT2_contig_contig00006_whole_enomée_shotgun_sequence

Geñome Set $4321^{-}$N/A N/A Actinobacteria

UMGS1205_ERR209515_bin.8_Bacteroidetes_Bacteroidia_Bacteroidales_Bacteroi daceae_Bäcteroides_NODE_507_length_25219_cov_43.399698 Genome Set

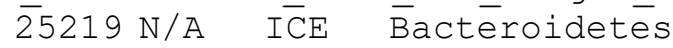

UMGS2056_SRR1761696_bin.44_Firmicutes_Clostridia_Clostridiales_Peptostrep

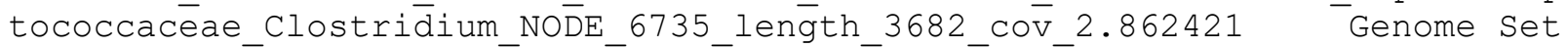

\section{$3682 \overline{\mathrm{N}} / \mathrm{A} \quad \mathrm{Plasmi} \overline{\mathrm{d}}$ Firmicutes}

NZ_LN908964.1_Ruminococcaceae_bacterium_AM2_genome_assembly_Fournierella_ massiliensis_scaffold_scaffold00019_whole_genome_shotgun_sequence

Genome Set $86151 \overline{5} \quad$ N/A ICE Firmicutes

NZ_EQ973635.1_Bacteroides_coprophilus_DSM_18228_=_JCM_13818_genomic_scaff

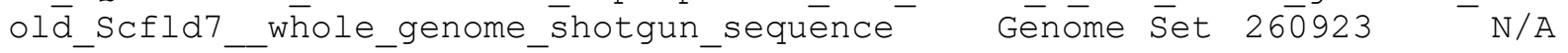

ICE Bacteroidetes

20298_2_7_Bacteroidetes_Bacteroidia_Bacteroidales_Bacteroidaceae_Bacteroi des_. $20 \overline{2} 9 \overline{8}$ 2_7.4 Genome Set $653472^{-}$N/A ICE ${ }^{-}$Bacteroidetes

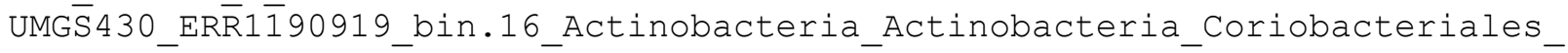

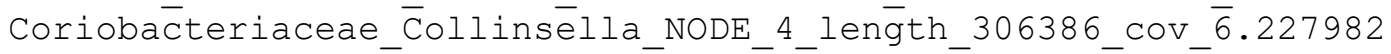

Genome set $\overline{3} 06386 \quad \bar{N} / \mathrm{A} \quad \overline{\mathrm{I}} \overline{\mathrm{CE}}$ Actinobactēia

12718_7_47_Firmicutes_Clostridia_Clostridiales_NA_NA_.12718_7_47.11

Genome set $2960 \overline{4}$ N/A ICE ${ }^{-}$Firmicutes

UMGS593_ERR911961_bin.38_Firmicutes_Clostridia_Clostridiales_Peptostrepto

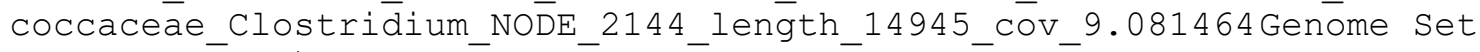

$149 \overline{45} \mathrm{~N} / \mathrm{A} \quad \mathrm{ICE}-$ Firmicutēes

21673_4_53_Firmicutes_Clostridia_Clostridiales_Lachnospiraceae_Blautia_.2 1673_4 $\overline{4} 3 . \overline{2}$ Genome Set 488635 - N/A ICE Firmicutes

21673_4_37_Firmicutes_Clostridia_Clostridiales_Lachnospiraceae_NA_.21673_ $437 . \overline{1} 6$ Genome set $61880 \mathrm{~N} / \mathrm{A}$ ICE Firmicutes

21̄673_4_3_Firmicutes_Clostridia_Clostridiales_Lachnospiraceae_NA_.21673_4 3.10 Genome set $800 \overline{7} 9 \mathrm{~N} / \mathrm{A}$ ICE Firmicutes

UMMGS683_ERR1190910_bin.59_Firmicutes_Clostridia_Clostridiales_oscillospir aceae_Oscillibacter $\bar{s}$ NODE_29_length_159983_cov_6.275061 Genome Set
159983
$\mathrm{N} / \overline{\mathrm{A}} \quad \mathrm{I} \overline{\mathrm{C}} \mathrm{E}$ Firmicutes

UMGS840_SRR3737012_bin.13_Bacteroidetes_Bacteroidia_Bacteroidales_Rikenel laceae_Ālistipes_NŌDE_13_Iength_219166_Cov_6.314325 Genome set $21 \overline{9} 166$

$\overline{\mathrm{N}} / \mathrm{A} \quad$ ICE Bacterōidetes 
13414_6_70_Firmicutes_Clostridia_Clostridiales_Lachnospiraceae_NA_.13414_ $670 . \overline{1}-$ Genome Set 304853 N/A ICE Firmicutes

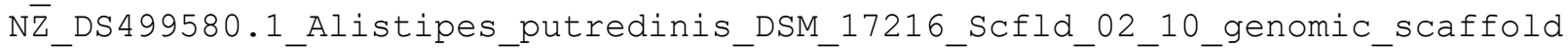
whole_genoméeshotgun_sequence Genome Set $4796 \overline{42}-{ }^{-}$N/A I $\overline{C E}$

Bäcteroidetes

21673_4_71_Firmicutes_Clostridia_Clostridiales_Peptostreptococcaceae_clos

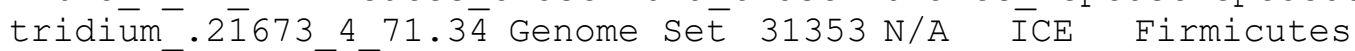

NZ_GL538343.1_Eaécalibacterium_cf._prausnitzii_KLE1255_genomic_scaffold_S cfīd592_wholèegenome_shotgun_sequence Genome- Set $923 \overline{10}$ N/A ICE

Firmicutes

13414_6_17_Firmicutes_Clostridia_Clostridiales_Lachnospiraceae_Ruminococc us_. $1 \overline{3} 4 \overline{1} 4 \overline{6} 17.3$ Genome Set $318 \overline{967}$ N/A $\overline{\text { ICE }}$ Firmicutes

UMGGS57_SRR̄2155361_bin.23_Firmicutes_Clostridia_Clostridiales_NA_NA_NODE_6 6 length $81507 \mathrm{cov} 10.11 \overline{8082}$ Genome set $81507 \overline{\mathrm{N}} / \mathrm{A}$ ICE Firmicutes

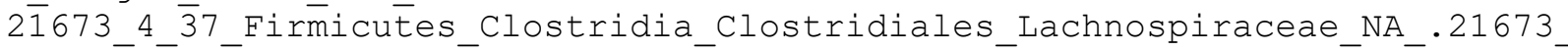
$437 . \overline{4}$ - Genome Set 215716 N/A ICE Firmicutes

$2 \overline{0} 427425$ Firmicutes Clostridia Clostridiales Lachnospiraceae Lachnobact

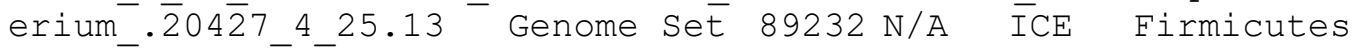

UMGS19̄11_ERR $\overline{9} 1 \overline{1} 953$ bin.70_Firmicutes_Clostridia_Clostridiales_Ruminococca ceae_NA_NoDE_2208_İength_18657_cov_3.586550 Genome Set $1865 \overline{7}$ N/A ICE

Firmicutes

18048_2_90_Bacteroidetes_Bacteroidia_Bacteroidales_Porphyromonadaceae_Odo ribacter_.'̄18048_2_90.14-Genome set 110735 N/A ICE

Bacteroidetes

21673471 Firmicutes Clostridia Clostridiales Peptostreptococcaceae Clos tridium_.21̄673_4_71.19 Genome Set 75918 N/A ICE Firmicutes

UMGS658_SRR215 $\overline{5} 3 \overline{8} 7$ bin.7_Firmicutes_Clostridia_Clostridiales_NA_NA_NODE_3 2_length̄ 165016 cov $19.8 \overline{6} 76$ Genome Set $165016^{-}$N/A ICE ${ }^{-} \overline{\text { Firmicutes }}$ UM̄GS 488 SRR5275456_bin.21_Firmicutes_Clostridia_Clostridiales_Lachnospira ceae_Dorea_NODE_44 $\overline{2}$ length_49732_cov_46.6453 Genome set $4973 \overline{2}$ N/A ICE Firmícutes

20298_2_31_Firmicutes_Negativicutes_Selenomonadales_Veillonellaceae_NA_.2 $0298 \overline{2} \overline{3} 1 . \overline{5}$ Genome Set 307045 N/A ICE Firmicutes

UMGS785_ERR1297697_bin.13_Firmicutes_Clostridia_Clostridiales_NA_NA_NODE_

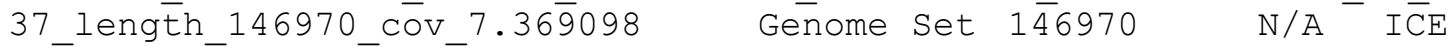

Firmicutes

14207_7_70_Bacteroidetes_Bacteroidia_Bacteroidales_Rikenellaceae_Alistipe

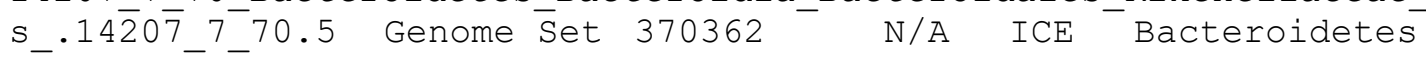

144207_7_89_Firmicutes_Clostridia_Clostridiales_NA_NA_.14207_7_89.25

Genome Set $5206 \overline{5}$ N/A ICE Firmicutes

20287_6_53_Bacteroidetes_Bacteroidia_Bacteroidales_Bacteroidaceae_Bactero

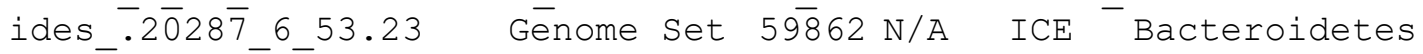
NZ_JH414884.1_Citrobacter_freundii_4_7_47CFAA_supercont1.9_whole_genome_

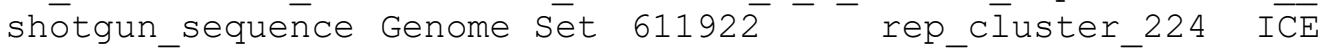

Proteobacteria

UMGS155_ERR688606 bin.32 Firmicutes Clostridia_Clostridiales Ruminococcac

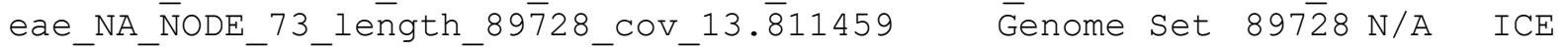
Firmicutes

UMGS1255 ERR1578662 bin.19 Tenericutes Mollicutes Erysipelotrichales Erys

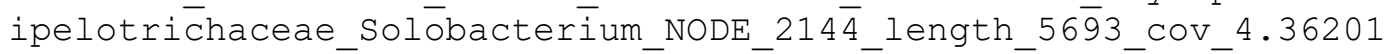

Genome Set 5693 N/A Plasmid Tenericutes

UMGS100_ERR1913101_bin.17_Bacteroidetes_Bacteroidia_Bacteroidales_Porphyr omonadac̄eae_NA_NODE_37_length_66993_cov_10.251740 Genome set $66 \overline{993}$ N/A ICE Bacteroidetes 
20298_2_10_Firmicutes_Bacilli_Lactobacillales_Streptococcaceae_Streptococ cus_. $\overline{2} 0 \overline{2} 98$ _2_10.9 Genome set 79895 N/A ICE Firmicutes

14207_7_28_Firmicutes_Clostridia_Clostridiales_NA_NA_.14207_7_28.1

Genome set $8208 \overline{4} 3 \quad$ N/A ${ }^{-}$ICE Firmicutes

UMGS248_ERR525690_bin.5_Firmicutes_Clostridia_Clostridiales_Lachnospirace ae_Robinsoniella_N̄ODE_8_length_209524_cov_37.4̄096 Genome set 209524

N/A ICE Firmicutes

NZ_KE340301.1_Prevotella_oralis_HGA0225_genomic_scaffold_acAqJ-

supercont1.1_whole_genome_shotḡun_sequēence Genome set 1311360 N/A

ICE Bacteroidetes

12718_7_73_Firmicutes_Clostridia_Clostridiales_Lachnospiraceae_NA_.12718_

$773 . \overline{1} 8^{-}$Genome set $36296 \mathrm{~N} / \mathrm{A}^{-}$ICE Firmicutes

200298_3_70_Tenericutes_Mollicutes_Erysipelotrichales_Erysipelotrichaceae_ NA $.2 \overline{0} 2 \overline{9} 8 \overline{3} 70.9$ Genome Set $1701 \overline{95}$ N/A ICE Tenericutes

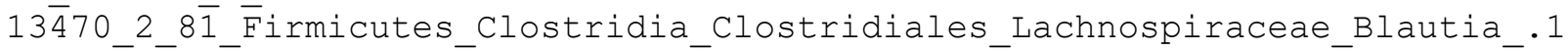

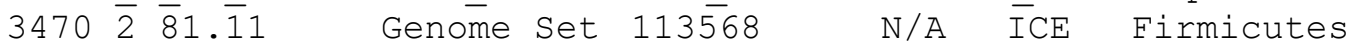

UMGS $\overline{3} 4 \overline{0}$ SRR1761708 bin.14 Firmicutes Clostridia Clostridiales Ruminococca

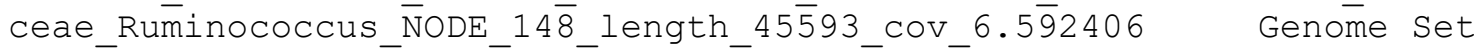

$45593 \mathrm{~N} / \mathrm{A} \quad \overline{\mathrm{I}} \mathrm{CE}$ Firmicutes

NZ_GL890556.1_Lachnospiraceae_bacterium_6_1_63FAA_genomic_scaffold_superc ont̄1.9_whole_genome_shotgun_sequence Gēōme Set $94685 \overline{\mathrm{N}} / \mathrm{A}$ ICE

Firmicutes

11861_6_63_Bacteroidetes_Bacteroidia_Bacteroidales_Rikenellaceae_Alistipe

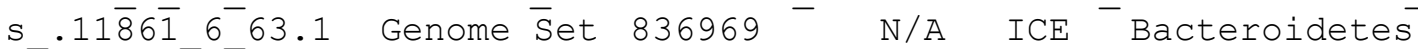

UMGGS1094_SRR5056760_bin.8_Actinobacteria_Actinobacteria_Coriobacteriales_ Coriobacteriaceae_Cōllinsēlla_NODE_1804_Iength_19819_cov_11.156193

Genome Set $\overline{1} 9819$ N/A ICE Actinōbactería

20298_3_34_Firmicutes_Clostridia_Clostridiales_NA_NA_.20298_3_34.4

Genome Set $4335 \overline{9} 9 \quad$ N/A ${ }^{-}$ICE Firmicutes

NZ_HE978648.1_Alistipes_sp._JC50_genomic_scaffold_scaffold00002_whole_g enome_shotgun_sequence Genome Set $173782 \overline{1}$ N/A ICE Bacteroidetes 13414_6_67_Firmicutes_Clostridia_Clostridiales_Lachnospiraceae_NA_.13414_ 6 67. ${ }^{-}$- Genome Set $313182-$ N/A ICE Firmicutes

UMMGS599_SRR5050586_bin.21_Firmicutes_Clostridia_Clostridiales_Ruminococca ceae_NA_NODE_112_lēngth_1̄̄2649_cov_4.761559 Genome Set $1126 \overline{4} 9$ N/A I $\overline{C E} \quad \overline{\text { Firmicutes }}$

13414_6_44_Firmicutes_Clostridia_Clostridiales_Lachnospiraceae_Lachnobact erium.$\overline{1} 34 \overline{1} 4644.13^{-}$Genome Set $84813 \mathrm{~N} / \mathrm{A}$ ICE Firmicutes

UMGS $8 \overline{7} 3$ ERR11 $9 \overline{0} 965$ bin.23 Firmicutes Clostridia_Clostridiales Lachnospira

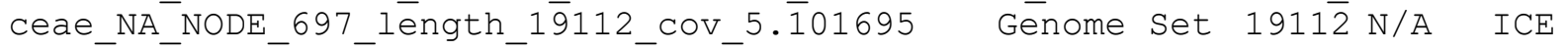

Firmicutes

13414_6_50_Firmicutes_Clostridia_Clostridiales_Lachnospiraceae_Dorea_.134 14 6_50.3 Genome Set 312755 - N/A ICE Firmicutes

NZ_GL891990.1_Dysgonomonas_gadei_ATCC_BAA-

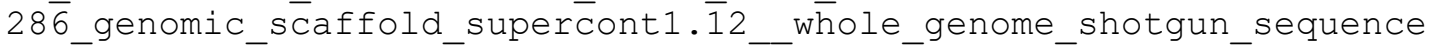
Genome set $132 \overline{1} 40 \quad$ N/A ICE Bacteroidetes

13470_2_73_Firmicutes_Negativicutes_Selenomonadales_Veillonellaceae_Megas

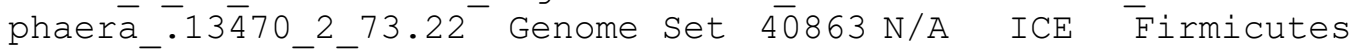

NZ DS483517.1 Parvimonas micra ATCC 33270 Scfld 0213 genomic scaffold W hole_genome_shotgun sequēnce Genome Set $5 \overline{05805}{ }^{-}{ }^{-} \bar{N}_{\mathrm{A}}$ ICE ${ }^{-}$Firmicutes 13414_6_53_Firmicutēs_Clostridia_Clostridiales_Ruminococcaceae_Faecalibac terium_.134̄14_6_53.13 Genome Set 86190 N/A ICE Firmicutes 
NZ_DS264285.1_Eubacterium_ventriosum_ATCC_27560_Scfld0223_genomic_scaffol d_whole_genome_shotgun_séquence Geñome Set $2 \overline{4} 7920 \quad \overline{\mathrm{N}} / \mathrm{A} \quad \mathrm{N} / \overline{\mathrm{A}}$ Firmicutes

NZ DS990134.1 Bacteroides plebeius DSM 17135 Scfld 0217 genomic scaffold whole_genoméshotgun_sequence Genome Set $722256-{ }^{-}$- N/A ICE

Bäcteroīetes

14207_6_3_Bacteroidetes_Bacteroidia_Bacteroidales_Porphyromonadaceae_NA_. $14207{ }^{-6}$-26 Genome Set $36969 \mathrm{~N} / \mathrm{A}$ ICE Bacteroidetes

13414_6_3_Firmicutes_Clostridia_Clostridiales_NA_NA_.13414_6_3.7 Genome set $250 \overline{9} 42$ N/Ā ICE Firmicutés

UMGS233_SRR6028305_bin.9_Firmicutes_Clostridia_Clostridiales_Ruminococcac eae_NA_N NODE_1466_lēength_6844_cov_12.659449 Genome set $684 \overline{4}$ N/A ICE Firmicutes

UMGS1899_ERR1913030_bin.47_NA_NA_NA_NA_NA_NODE_8646_length_3247_COV_3.760 025 Geñome set $32 \overline{4} 7 \mathrm{~N} / \mathrm{A}^{-} \overline{\mathrm{ICE}}-\overline{\mathrm{NA}}$

UMGS78_ERR1190897_bin.8_Firmicutes_Clostridia_Clostridiales_Lachnospirace ae_Rosēburia_NODE_83_length_128967_Cov_18.9794̄20 Genome Set 128967

N/A ICE Firmicutes

NZ_KB905470.1_Bacteroides_salyersiae_WAL_10018_=_DSM_18765_=_JCM_12988_ge

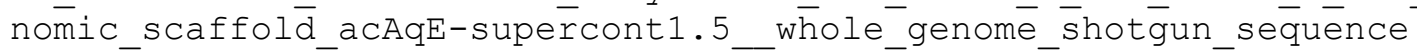
Genome set $116045 \quad$ N/A $\overline{\text { ICE }}$ Bacteroidetes

UMGS143_ERR688524_bin.28_Firmicutes_Clostridia_Clostridiales_Lachnospirac

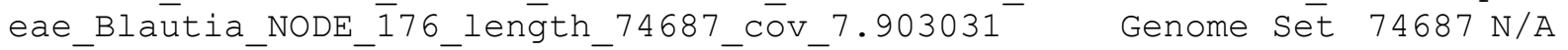
ICE Firmicutes

NZ_EQ973637.1_Bacteroides_coprophilus_DSM_18228_=_JCM_13818_genomiC_scaff old̄_Scfld9_whole_genome_shotgun_sequence ${ }^{-}$Genome $\overline{\text { Set }} 24 \overline{7} 317$ - N/A ICE Bacteroidetes

13470_2_93_Firmicutes_Clostridia_Clostridiales_Lachnospiraceae_NA_.13470_ 2 93. 32 - Genome Set $29126 \mathrm{~N} / \mathrm{A}^{-}$ICE Firmicutes

UM̄GS1624_ERR688644_bin.6_Bacteroidetes_Bacteroidia_Bacteroidales_Prevotel laceae_Prevotella_N̄ODE_125_length_80515_cov_27.737547 Genome $\overline{\text { Set }}$

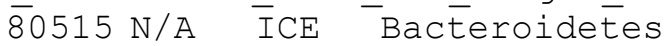

20427_4_25_Firmicutes_Clostridia_Clostridiales_Lachnospiraceae_Lachnobact erium_. $204 \overline{2} 7$ 4_25.149 Genome Set 502 N/A Plasmid Firmicutes

UMGS1531_ERR $\overline{5} 2 \overline{5} 788$ bin.2_Firmicutes_Clostridia_Clostridiales_Peptostrepto

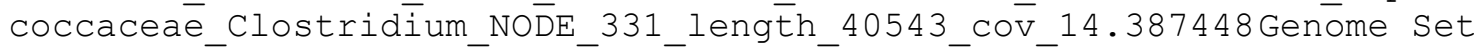
$405 \overline{4} 3 \mathrm{~N} / \mathrm{A} \quad \mathrm{N} / \mathrm{A}$ - Firmicutes

UMGS692_ERR1913113_bin.1_Firmicutes_Clostridia_Clostridiales_NA_NA_NODE_1 7 length_.93063_cov_10.188199 Genome Set 93063 N/A ICE Firmicutes UM̄GS1598_ERR414334_bin.22_Bacteroidetes_Bacteroidia_Bacteroidales_Prevote

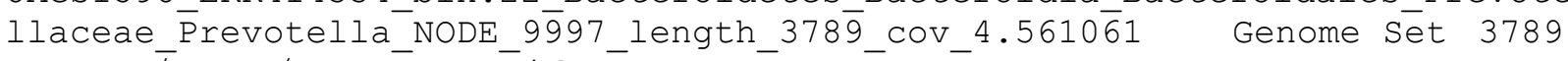
N/A N/A Bäcteroidetēs

NZ_ABJL02000008.1_Bacteroides_intestinalis_DSM_17393_B_intestinalis2.0.1_Cont607_whöle_genome_shotgun_sequence Genome $\overline{\text { Set }} 3472993$ N/A ICE Bacteroidetes

UMGS834_ERR1620269_bin.44_Firmicutes_Clostridia_Clostridiales_NA_NA_NODE

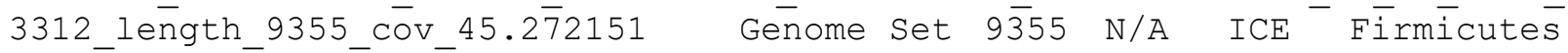

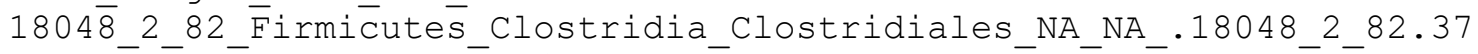
Genome set $2987 \overline{6}$ N/A ICE Firmicutes

NZ_GL698429.1_Streptococcus_equinus_ATCC_9812_genomic_scaffold_SCAFFOLD1_ _whole_genome_shotgun_sequence Genome ${ }^{-}$Set ${ }^{-} 1382815^{-}$N/A ${ }^{-}$ICE Firmicutes 
UMGS133_ERR209556_bin.16_Firmicutes_Clostridia_Clostridiales_Ruminococcac

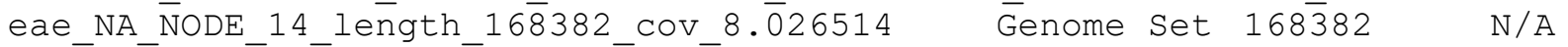
ICE Firmicutes

13414_6_15_Firmicutes_Clostridia_Clostridiales_Lachnospiraceae_NA_.13414_ 6 15.17 ${ }^{-}$Genome Set 85325 N/A ICE Firmicutes

21673_4_18_Firmicutes_Clostridia_Clostridiales_NA_NA_.21673_4_18.4

Genome set $2333 \overline{1} 8 \quad$ N/A ICE Firmicutes

8080_1_78_Firmicutes_Clostridia_Clostridiales_Peptostreptococcaceae_Clost

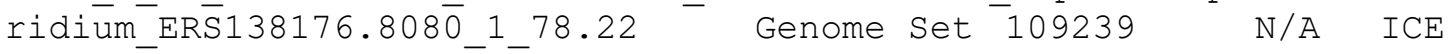

Firmicutes

14207_7_10_Firmicutes_Clostridia_Clostridiales_Lachnospiraceae_NA_.14207_ $710 . \overline{2}-$ Genome Set 735448 - N/A ICE Firmicutes

200298_3_39_Firmicutes_Clostridia_Clostridiales_NA_NA_.20298_3_39.31

Genome set $1778 \overline{6}$ N/A ICE ${ }^{-}$Firmicutes

UMGS54_SRR3131812_bin.3 Proteobacteria_Gammaproteobacteria_Enterobacteria les_Enterobacteriāceae_Pantoea_NODE_27_length_85418_cov_34.003034

Genome Set 85418 IncFII - Plasmid Proteobacteria

UMGS695_ERR1913046_bin.9_Tenericutes_Mollicutes_Erysipelotrichales_Erysip

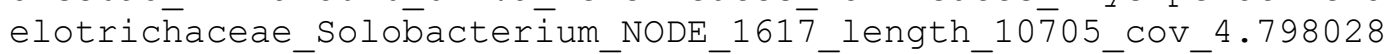

Genome set 10705 N/A ${ }^{-}{ }_{\text {ICE }}{ }^{-}$Tenericutes

21673_4_12_Firmicutes_Clostridia_Clostridiales_Lachnospiraceae_NA_.21673

$412 . \overline{6}-$ Genome Set $244387-$ N/A ICE Firmicutes

UM̄GS1011_ERR414329_bin.39_Firmicutes_Clostridia_Clostridiales_NA_NA_NODE 131 length 55239 Cov 5.025913 Genome set $5 \overline{5239}$ N/A ICE Firmícutes 8080_1_78_Firmicutes_Clostridia_Clostridiales_Peptostreptococcaceae_Clost ridium_ERS̄138176.8080_1_78.19- Genome Set 133873 N/A ICE

Firmicutes

UMGS 446 SRR2674235 bin.2_Actinobacteria Actinobacteria_Coriobacteriales_C oriobactereriaceae_Cōllinsēlla_NODE_1603_Iength_25338_cō̄_6.676186

Genome Set 25338 N/A ICE Actinobacteria

20298_2_7_Bacteroidetes_Bacteroidia_Bacteroidales_Bacteroidaceae_Bacteroi des_. $\overline{2} 0 \overline{2} 9 \overline{8}{ }_{2}$ 7.1 Genome Set $979168^{-}$N/A ICE ${ }^{-}$Bacteroidetes 14207_7_42_Firmicutes_Clostridia_Clostridiales_Lachnospiraceae_NA_.14207_ $742 . \overline{1}-$ Genome Set $308594 \quad$ N/A ICE Firmicutes NZ__KE161030.1_Cedecea_davisae_DSM_4568_Scaffold0_whole_genome_shotgun_se quence Genome Set 4876800 N/A ICE Proteobacteria

UMGS1625_ERR414305_bin.19_Firmicutes_Clostridia_Clostridiales_Peptostrept

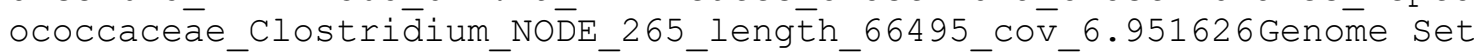

$6649 \overline{5} \mathrm{~N} / \mathrm{A}$ ICE Firmicutēes

20287_6_22_Firmicutes_Clostridia_Clostridiales_Ruminococcaceae_Ruminococc us_.20287 $\overline{6}$ 22.1 Genome Set $108 \overline{8099}$ N/A ICE Firmicutes

UMGGS1255_ERR 1578662 bin.19_Tenericutes_Mollicutes_Erysipelotrichales_Erys ipelotrichaceae_Solōbacterīum_NODE_2430_length_487̄2_cov_5.54723

Genome Set 4872 N/A Plasmid Tenericutes

NZ_DS990260.1_Clostridiales_bacterium_1_7_47_FAA_supercont2.1_genomic_sca fföld_whole_ḡenome_shotgun_sequence - Génome sēt 3013027 N/A ICE

Firmicutes

8080_1_80_Tenericutes_Mollicutes_Erysipelotrichales_Erysipelotrichaceae_S olobācterium_ERS138178.8080_1_80.4 Genome Set $19117 \overline{6}$ N/A ICE

Tenericutes

NZ_LT559262.1_Alistipes_sp._Marseille-

P2431_whole_genome_shotgun_sequence Genome set 3788833 N/A ICE

Bacteroidetes 
NZ_GG657587.1_Clostridium_asparagiforme_DSM_15981_genomic_scaffold_Scfld1 whole_genomé_shotgun_sequence Genome set $823 \overline{3} 88 \quad \overline{\mathrm{N}} / \mathrm{A}$ ICE Firmicutes

UMGS1925 SRR5275467 bin.39 Firmicutes Clostridia Clostridiales Peptostrep

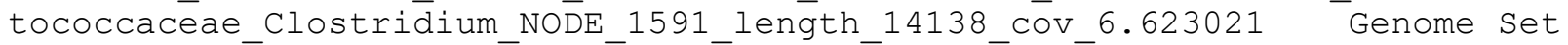
$14138 \overline{\mathrm{N}} / \mathrm{A} \quad \mathrm{N} / \mathrm{A} \quad \overline{\mathrm{F}} \mathrm{irmi} \bar{c}$ utes

NZ_DS483502.1_Faecalibacterium_prausnitzii_M21_2_Scfld_02_23_genomic_scaf fold_whole_gēnome_shotgun_sequence Geñome sēt $207415-$ N/A ICE Firmicutes

NZ_KI517369.1_Pseudomonas_sp._HPB0071_genomic_scaffold_aczIi-

supercont1.3_whole_genomé_shötgun_sequence Genome sēt 318103 N/A N/A Proteobacteria

NZ_FCEY01000006.1_Clostridium_sp._AT5_genome_assembly_contig_contig00006 whole genome shōtgun sequence - Genome set 617428 N/A ICE Firmicutes

UMGS1733_ERR011115_bin.6_Bacteroidetes_Bacteroidia_Bacteroidales_Prevotel

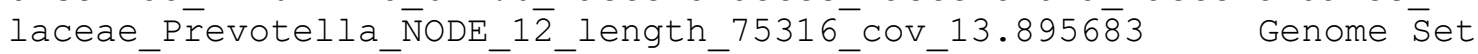

$75316 \mathrm{~N} / \mathrm{A} \quad \overline{\mathrm{I}} \mathrm{CE}$ Bacteroidetes

UMGS1028_SRR341596_bin.15_Tenericutes_Mollicutes_Erysipelotrichales_Erysi pelotrichaceae_Solōbacterium_NODE_65_İength_74596_cov_2.987336 Genome Set $74596 \mathrm{~N} / \mathrm{A}^{-} \quad \mathrm{N} / \mathrm{A}$ Tenericutes

NZ_DS264265.1_Eubacterium_ventriosum_ATCC_27560_Scfld023_genomic_scaffold whole_genomé_shotgun_sequence Geñome Set $30 \overline{0} 9531$ N/A I $\bar{C} E$ Firmicutes

13414_6_67_Firmicutes_Clostridia_Clostridiales_Lachnospiraceae_NA_.13414 $667 . \overline{5} 6^{-}$- Genome Set 630 N/A ${ }^{-}$N/A Firmicütes

UM̄GS660 ERR414279 bin.17 Firmicutes Clostridia Clostridiales Eubacteriace

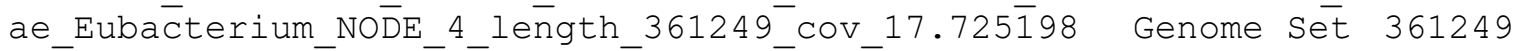

N/A ICE Firmicutes

21673_4_18_Firmicutes_Clostridia_Clostridiales_NA_NA_.21673_4_18.13

Genome set $9393 \overline{8}$ N/A ICE ${ }^{-}$Firmicutes

NZ_GL945333.1_Lachnospiraceae_bacterium_2_1_58FAA_genomic_scaffold_superc

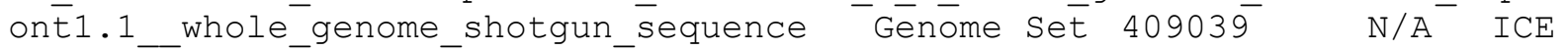
Firmicutes

20427_4_25_Firmicutes_Clostridia_Clostridiales_Lachnospiraceae_Lachnobact erium_. $204 \overline{2} 7$ 4_25.89 Genome Set 2786 N/A N /A Firmicutes NZ_KI $\overline{3} 91949 . \overline{1}$ R Ruminococcaceae_bacterium_D16_genomic_scaffold_acsPysupercont2.3_whole_genome_shotgun_sequence Genome Set $269 \overline{9} 07$ N/A ICE Firmicutes

UMGS1057_SRR6028203_bin.16_Verrucomicrobia_Opitutae_Opitutales_NA_NA_NODE _233_lenḡth_28920_cōov31.0̄49818 Genome Set 28920 N/A ICE

Verrucomicrōbia

14207_7_23_Bacteroidetes_Bacteroidia_Bacteroidales_Bacteroidaceae_Bactero ides_.14 $20 \overline{7}$ 7_23.63 Gēnome set $48 \overline{9} 1$ N/A Plasmid Bacteroídetes

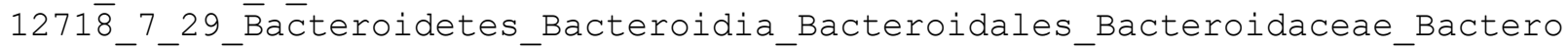

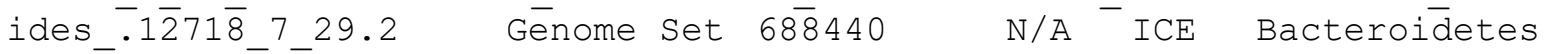
UMGST18_SRR12 $1 \overline{5} 278$ _bin.2_Bacteroidetes_Bacteroidia_Bacteroidales_Porphyrom onadacēae_Porphyromonas_NODE_19_length_63799_cov_ $\overline{6} .688896 \mathrm{Genome}$ - Set $637 \overline{9} 9 \mathrm{~N} / \mathrm{A}$ ICE Bacteroidetes

20298_3_69_Firmicutes_Clostridia_Clostridiales_Ruminococcaceae_NA_.20298_ 3_69.8 - Genome Set 192319 - N/A ICE Firmicutes UM̄GS472_ERR1578619_bin.63_Firmicutes_Clostridia_Clostridiales_Lachnospira

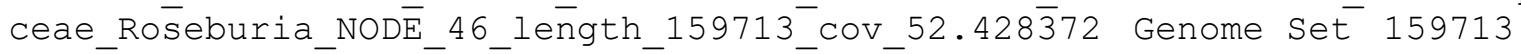
N/A ICE Firmicutes 
NZ_HG005294.1_Erysipelotrichaceae_bacterium_AP9_genomic_scaffold_scaffol d00011_whole_genome_shotgun_sequēnce Genome Set $106591 \quad$ N/A N/A Tenericutes

20287653 Bacteroidetes Bacteroidia Bacteroidales Bacteroidaceae Bactero ides_.202877_6_53.13 Gēnome set $24 \overline{4} 799$ N/A ICE Bacteroidetes 12718_7_73_Firmicutes_Clostridia_Clostridiales_Lachnospiraceae_NA_.12718_ $773 . \overline{1} 2^{-}$- Genome set $254429-$ N/A ICE Firmicutes

13414_6_17_Firmicutes_Clostridia_Clostridiales_Lachnospiraceae_Ruminococc us_.13 $4 \overline{1} 4 \bar{\sigma}_{1} 17.13 \mathrm{Genome}$ Set $115 \overline{3} 66$ N/A ICE Firmicutes

UMGGS1213_STRR5558409_bin.19_Bacteroidetes_Bacteroidia_Bacteroidales_Bacter

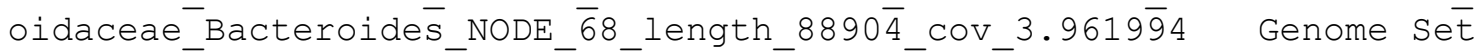
$88 \overline{9} 04 \mathrm{~N} / \mathrm{A} \quad \mathrm{ICE}^{-}$Bacteréroidetes

NZ_DS483477.1_Eubacterium_dolichum_DSM_3991_Scfld_02_17_genomic_scaffold_ _whole_genome_shotgun_sequence Genome set $453733^{-}$- N/A ICE Tenericutes

UMGS235_ERR526047_bin.37_Actinobacteria_Actinobacteria_Coriobacteriales C

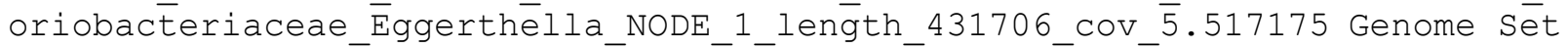
$431706 \quad$ N/A N/A Actiñobacteria

NZ_DS990262.1_Clostridiales_bacterium_1_7_47_FAA_supercont2.3_genomic_sca ffōld_whole_genome_shotgun_sequence - $-\bar{G} \overline{-}$ nome sēt $992254 \quad$ N/A ICE Firmicutes

UMGS697_SRR5056891_bin.33_Firmicutes_Clostridia_Clostridiales_Lachnospira ceae_NA_NODE_279_lēength_5 $\overline{5} 760 \_$cov_32 $\overline{5} .626784$ Genome set $5576 \overline{0}$ N/A ICE Fírmicutes

NZ_DS990119.1_Bacteroides_plebeius_DSM_17135_Scfld_02_2_genomic_scaffold_ _whole_genome_shotgun_sequence Genome Set $5712 \overline{8} 8-{ }^{-}-$N/A $\overline{\text { ICE }}$ Bacteroidetes

20298_3_58_Firmicutes_Clostridia_Clostridiales_NA_NA_.20298_3_58.8 Genome Set $2183 \overline{0} 1 \quad$ N/A ${ }^{-}$ICE Firmicutes

NZ_JH414766.1_Tannerella_sp._6_1_58FAA_CT1_genomic_scaffold_supercont1.1 -whole_genome_shotgun_sequence ${ }^{-}$Genome Set $1024 \overline{172}$ rep_cluster_170 ICE Bacteroidetes

20298_3_30_Bacteroidetes_Bacteroidia_Bacteroidales_Porphyromonadaceae_NA_ $.2029 \overline{8} \overline{3} 3 \overline{0} .1 \quad$ Genome $\overline{\text { Set }} 556069^{-}$N/A ICE Bacteroidetes

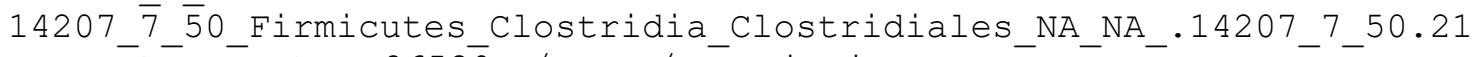
Genome Set $2653 \overline{9}$ N/A N/A Firmicutes

NC_020555.1_Helicobacter_cinaedi_CCUG_18818_=ATCC_BAA$84 \overline{7}$ DNA_complete_genome Genome set $224 \overline{0} 1 \overline{3} 0$ N/A ICE Proteobacteria

UMGS1806_SRR5279238_bin.24_Firmicutes_Clostridia_Clostridiales_Lachnospir

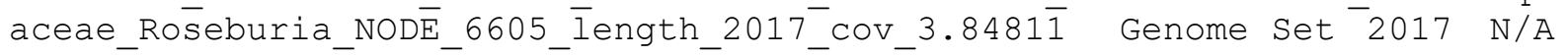
N/A Firmicutes

20298_3_17_Proteobacteria_Gammaproteobacteria_Enterobacteriales_Enterobac

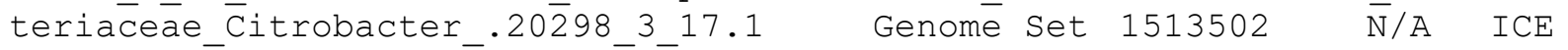
Proteobacteria

NZ_HE578952.1_Enterobacter_massiliensis_JC163_strain_type_strain_JC163 whöle_genome_shotgun_sequeñce Genome set 91086 N/A Plasmid Proteobacteria

NZ_KE993663.1_Clostridium_sp._ATCC_29733_genomic_scaffold_Scaffold88_who le_genome_shot̄gun_sequence Genome Set $\overline{9} 1831 \mathrm{~N} / \overline{\mathrm{A}}$ ICE $\overline{\mathrm{F}}$ irmicutes NZ_CBYL010000049.1__Clostridium_jeddahense_strain_JCD_whole_genome_shotgu n_sequence Genome Set 22077 N/A ICE Fírmicutēs 13̄14_6_14_Firmicutes_Clostridia_Clostridiales_NA_NA_.13414_6_14.6 Genome set $1884 \overline{0} 8 \quad$ N/A ${ }^{-}$ICE Firmicutes 
21673_4_87_Firmicutes_Clostridia_Clostridiales_Lachnospiraceae_Dorea_.216 $734 \overline{8} 7.3$ - Genome Set 451377 N/A ICE Firmicutes

21 $\overline{6} 7 \overline{3}$ 4_87_Firmicutes_Clostridia_Clostridiales_Lachnospiraceae_Dorea_.216 $734 \overline{8} 7.15^{-}$Genome Set $46858 \mathrm{~N} / \mathrm{A}^{-}$ICE Firmicütes

NZ_EQ973222.1_Bacteroides_fragilis_3_1_12_supercont1.10_genomic_scaffold_ _whole_genome_shotgun_sequence_Genome Set 49314 N/A ICE

Bacteroidetes

13414_6_20_Firmicutes_Clostridia_Clostridiales_Lachnospiraceae_Blautia_.1 $3414 \overline{6} \overline{2} 0 . \overline{5}$ Genome Set $229452-$ N/A ICE Firmicutes

NZ_JḦ376874.1_Clostridium_clostridioforme_2_1_49FAA_genomic_scaffold_supe rcōnt1.5_whole_genome_shötgun_sequence Gēōomē set 313771 N/A ICE Firmicutes

NZ_JH414885.1_Citrobacter_freundii_4_7_47CFAA_supercont1.10_whole_genome

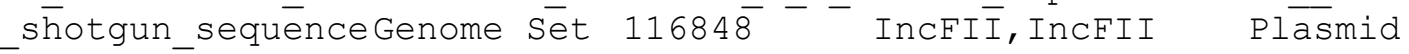

Proteobacteria

NC_015697.1_Lactobacillus_reuteri_SD2112_complete_genomeGenome Set $22643 \overline{9} 9 \quad$ N/A ICE Firmicutes

UMGS530_SRR2155369_bin.7_Firmicutes_Clostridia_Clostridiales_NA_NA_NODE_6 5_length_69452_cov_20.2657 Genome Set 69452 N/A ICE Firmicutes

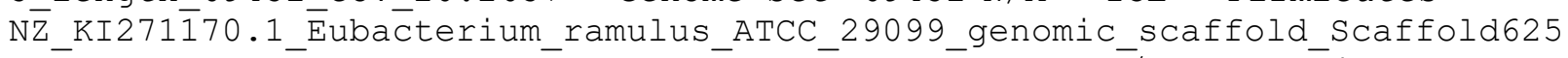
whole_genomé_shotgun_sequence - Genome Set 548 N/A Plasmid Firmicutes

12718_7_94_Firmicutes_Clostridia_Clostridiales_Lachnospiraceae_NA_.12718_ 7 94. ${ }^{-}$- Genome Set 441227 - N/A ICE Firmicutes

UMMGS219 ERR321453 bin.7 Bacteroidetes Bacteroidia Bacteroidales Bacteroid

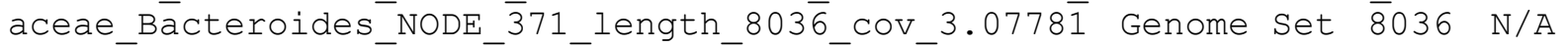
ICE Bacteroidetes

UMGS 489 SRR1761687 bin.58_Bacteroidetes_Bacteroidia_Bacteroidales Porphyr omonadac̄eae_NA_NODĒ_6_lenḡth_294183_cov_14.547041 Genome Set $29 \overline{4} 183$

N/A ICE Bactéroidetés

UMGS1642_SRR5275432_bin.18_Firmicutes_Clostridia_Clostridiales_Peptostrep

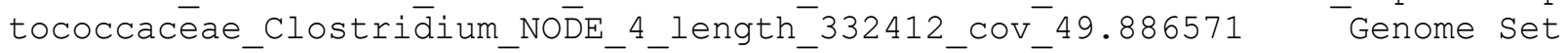
$33241 \overline{2} \quad$ N/A ICE Firmicutes

UMGS462_ERR1305881_bin.83_Firmicutes_Clostridia_Clostridiales_NA_NA_NODE_ 2121 length 18060 Cov $8.5 \overline{6} 9453$ Geñome set $1 \overline{8060}$ N/A ICE Fírmícutes UMGST̄345_ERR $\bar{R} 50509 \overline{8}$ bi $\bar{n} .47$ Tenericutes_NA_NA_NA_NA_NODE_11_length_145565_C ov_30.7957446 Genome Set $145565-\overline{\mathrm{N}} / \mathrm{A}^{-} \overline{\mathrm{N}} / \mathrm{A}^{-}$Tenericutes

NZ_ACFY01000051.1_Roseburia_inulinivorans_DSM_16841_R_inulinivorans-

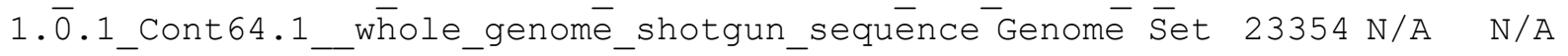

Firmicutes

12718_7_50_Proteobacteria_Gammaproteobacteria_Enterobacteriales_Enterobac teriacēeàe_Enterobacter_.12718_7_50.2 Genomē Set 1434765 N/A ICE

Proteobacteria

20287 6_9_Bacteroidetes_Bacteroidia_Bacteroidales_Bacteroidaceae_Bacteroi des_. $20 \overline{2} 8 \overline{7} 6$ 6.3 Genome Set $463550^{-}$N/A ICE ${ }^{-}$Bacteroidetes

142077737-Firmicutes_Clostridia_Clostridiales_Lachnospiraceae_Dorea_.142 07 7_ 37.8 - Genome Set 148313 - N/A ICE Firmicutes

NZ_LT632322.1_Murdochiella_sp._Marseille-P2341_strain_Marseille-

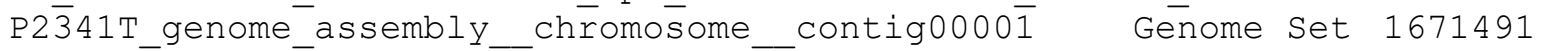

$\overline{\mathrm{N}} / \mathrm{A} \quad \mathrm{ICE}$ Firmicutes

14207_6_9_Bacteroidetes_Bacteroidia_Bacteroidales_Bacteroidaceae_Bacteroi des_. $14 \overline{2} 0 \overline{7} \_6$ 9.13Genome Set 87135 N/A ICE Bacteroidetes

21673_4_18_Firmicutes_Clostridia_Clostridiales_NA_NA_.21673_4_18.19

Genome Set $5656 \overline{1}$ N/A ICE ${ }^{-}$Firmicutes 
20427_4_25_Firmicutes_Clostridia_Clostridiales_Lachnospiraceae_Lachnobact

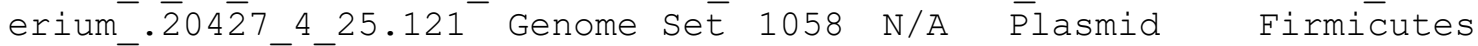
UMGS $1 \overline{6} 25$ ERR $\overline{4} 1 \overline{4} 305$ bin.19 Firmicutes Clostridia Clostridiales Peptostrept

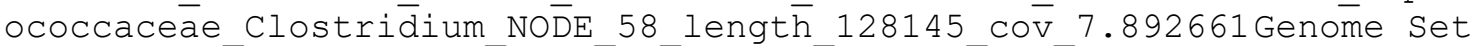
$1281 \overline{4} 5$
N/A ICE Firmicutes

18391_1_7_Actinobacteria_Actinobacteria_Bifidobacteriales_Bifidobacteriac

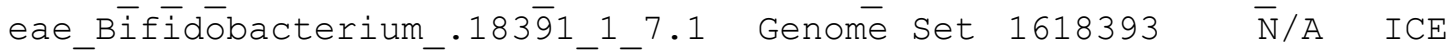
Actinobacteria

NZ_AEXR01000024.1_Eggerthella_sp._HGA1_contig00026_whole_genome_shotgun sequence Genome set $163482^{-}{ }^{-}$N/A ${ }^{-}$ICE Actinobacteria UMGS1646_SRR6028191_bin.15_Bacteroidetes_Bacteroidia_Bacteroidales_Bacter

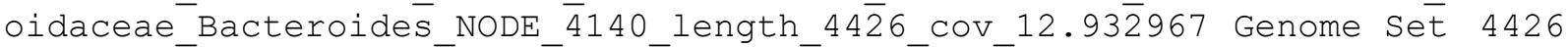
$\mathrm{N} / \overline{\mathrm{A}} \quad$ Plasmid - Bacteroìidetes

UMGS1607 SRR6054490 bin.8 Firmicutes Clostridia Clostridiales Lachnospira ceae_NA_NODE_380_leñgth_24549_cov_7. 863967 Genome set $2454 \overline{9}$ N/A ICE Firmicutes

UMGS1015_ERR1620298_bin.6_Firmicutes_Clostridia_Clostridiales_Peptostrept

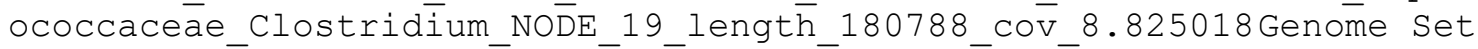
$1807 \overline{8} 8$
N/A ICE Firmicutes

13414_6_70_Firmicutes_Clostridia_Clostridiales_Lachnospiraceae_NA_.13414_ $670 . \overline{1} 7^{-}$- Genome Set $52692 \mathrm{~N} / \mathrm{A}^{-}$ICE Firmicütes

14̄207_7_9_Firmicutes_Clostridia_Clostridiales_Lachnospiraceae_Lachnospira $.142 \overline{0} 7^{-} 7-9.12$ Genome set $87 \overline{9} 38$ N/A ICE Firmicutes

$\overline{1} 4207$ 6_12 Bacteroidetes_Bacteroidia_Bacteroidales_Rikenellaceae_Alistipe

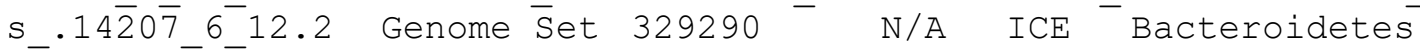

20287_6_22_Firmicutes_Clostridia_Clostridiales_Ruminococcaceae_Ruminococc us. $2 \overline{0} 2 \overline{8} 7 \overline{6} 22.18 \mathrm{Genome}$ Set $684 \overline{9}$ N/A N/A Firmicutes

20287_6_37_Firmicutes_Clostridia_Clostridiales_NA_NA_.20287_6_37.1

Genome set $2484 \overline{9} 3$ N/A ICE Firmicütes

UMGS607_ERR526073_bin.39_Bacteroidetes_Flavobacteriia_Flavobacteriales_Fl

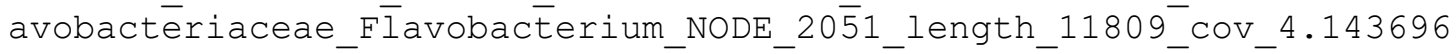

Genome Set 11809 N/A ICE Bacteroidetes

UMGS40_SRR2912796_bin.41_Firmicutes_Clostridia_Clostridiales_Ruminococcac

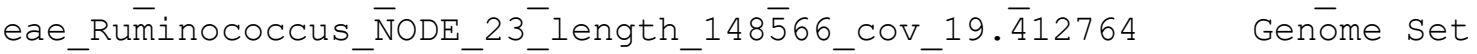
$148566-$ N/A I $\overline{C E}$ Firmicutes

14207_7_54_Firmicutes_Clostridia_Clostridiales_NA_NA_.14207_7_54.5 Genome set $2121 \overline{1} 4 \quad$ N/A ICE Firmicutes

UMGS1205_ERR209515_bin.8 Bacteroidetes Bacteroidia_Bacteroidales_Bacteroi

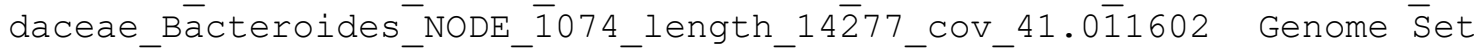

$\overline{1} 4277 \mathrm{~N} / \mathrm{A} \quad \mathrm{IC} E$ Bacteroidetes

21673_4_19_Firmicutes_Clostridia_Clostridiales_Lachnospiraceae_Blautia_.2 $1673 \overline{4} \overline{1} 9 . \overline{1} 5 \quad$ Genome Set $119 \overline{965}$ N/A ICE Firmicutes

14207_7_28_Firmicutes_Clostridia_Clostridiales_NA_NA_.14207_7_28.6

Genome set $2238 \overline{1} 0 \quad$ N/A ICE Firmicutes

NZ_HE978634.1_Noviherbaspirillum_massiliense_JC206_whole_genome_shotgun sequence Genome set 3152253 - N/A ICE Proteobacteria

NZ_GL945334.1_Lachnospiraceae_bacterium_2_1_58FAA_genomic_scaffold_superc ont̄1.2_whole_genome_shotgun_sequence Genome Set̄ $405671^{-}$N/A ${ }^{-}$ICE Firmicutes

NZ_ACFY01000044.1_Roseburia_inulinivorans_DSM_16841_R_inulinivorans-

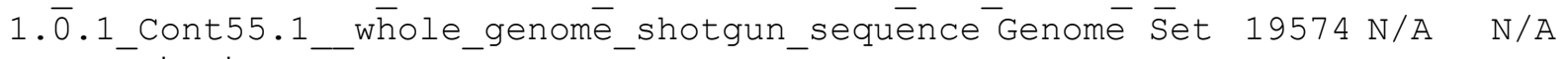
Firmicutes

NZ_DS547020.1_Clostridium_sp._SS2_1_Scfld_03_24_genomic_scaffold_whole_g enōme_shotgun_sequence Geñome ${ }^{-}$Set $^{-} \overline{11}_{16654}{ }^{-}{ }_{\text {N/A }}$ ICE ${ }^{-}$Firmicutes 
13470_2_92_Firmicutes_Clostridia_Clostridiales_Lachnospiraceae_NA_.13470_

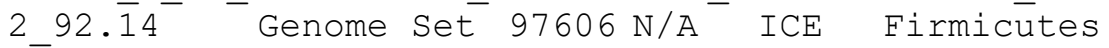
NZ__AEXR01000037.1_Eggerthella_sp._HGA1_contig00009_whole_genome_shotgun sequence Genome set $46243 \mathrm{~N} / \mathrm{A}$ ICE Actinobacteria

NZ_GL834361.1_Clostridium_symbiosum_WAL-

14 $\overline{6} 73$ genomic_scaffold_supercont1.5_whole_genome_shotgun_sequence Genome Set $42710 \overline{0}$ N/A ICE Firmicutes

NZ_LN869529.1_Intestinimonas_sp._GD2_genome_assembly_Intestinimonas_massi liēnsis_scaffold_scaffold00007_whole_genome_shotgun_sequence Genome Set $2 \overline{281024} \overline{\mathrm{N}} / \mathrm{A} \quad \mathrm{ICE} \quad$ Firmicutes

UMGS1471_ERR414252_bin.28_Firmicutes_Clostridia_Clostridiales_NA_NA_NODE_

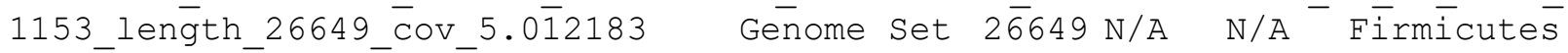
NZ_GI987988.1_Fusōbacterium_mortiferum_ATCC_9817_genomic_scaffold_superco

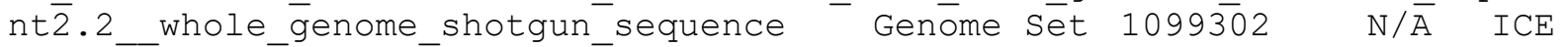

Fusobacteria

NZ_ACFY01000031.1_Roseburia_inulinivorans_DSM_16841_R_inulinivorans1.0.1_Cont39.1_whole_genomé_shotgun_sequence Genome $\overline{\text { Set }} 85784$ N/A ICE Firmicutes

8080_1_73_Firmicutes_Clostridia_Clostridiales_Peptostreptococcaceae_Clost ridium_ERS̄ $138171.808 \overline{0}{ }_{1}{ }_{-} 73.4$ Genome Set $37304 \overline{8}$ N/A ICE Firmicutes 20287_- 6 53_Bacteroidetes_s_Bacteroidia_Bacteroidales_Bacteroidaceae_Bactero

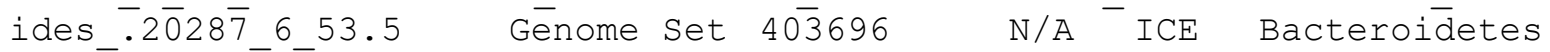
NZ_JḦ376871.1_Clostridium_clostridioforme_2_1_49FAA_genomic_scaffold_supe rcont1.2_whole_genome_shotgun_sequence Genome set 1202118 N/A ICE Firmicutes

UMGS1437_ERR209778_bin.10_Firmicutes_Clostridia_Clostridiales_Eubacteriac

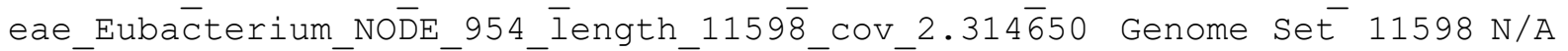

ICE Firmicutes

20298_3_57_Actinobacteria_Actinobacteria_Coriobacteriales_Coriobacteriace

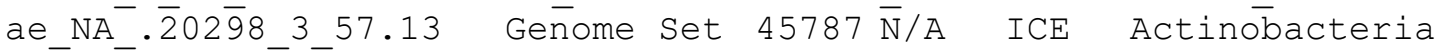
UMGGS308_SRR4 $\overline{3} 0 \overline{5} 073$ bin.1_Actinobacteria_Actinobacteria_Coriobacteriales_C oriobacteriaceae_Cōllinsēlla_NODE_5_length_138764_cov_ē25.489074

Genome set $138764 \quad \bar{N} / \mathrm{A}-\overline{\mathrm{IC}} \mathrm{C}$ Actinobacteriā

UMGS1501_SRR5127533_bin.13_Actinobacteria_Actinobacteria_Coriobacteriales _Coriobacteriaceae_Collinsēlla_NODE_47_length_74876_cov_2 24.555940

Genome set $7 \overline{4} 876$ N/A ICE Actinobacteria

UMGS50_ERR1293691_bin.12_Bacteroidetes_Bacteroidia_Bacteroidales_Rikenell aceae_Ālistipes_NODE_18_length_160958_Cov_30.067196 Genome Set $1 \overline{60958}$

N/A ICE ${ }^{-}$Bactereröidetes

NZ_GG661974.1_Helicobacter_winghamensis_ATCC_BAA-

$43 \overline{0}$ _genomic_scaffold_supercont1.2_whole_genome_shotgun_sequence Genome set $583 \overline{1} 00 \quad$ N/A ICE Proteobacteria

20298_3_21_Actinobacteria_Actinobacteria_Actinomycetales_Actinomycetaceae

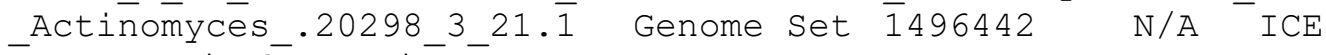
Actinobacteria

21673_4_12_Firmicutes_Clostridia_Clostridiales_Lachnospiraceae_NA_.21673_ $412 . \overline{1}{ }^{-}$Genome Set $147821-$ N/A ICE Firmicutes

13414_6_53_Firmicutes_Clostridia_Clostridiales_Ruminococcaceae_Faecalibac terium_.134 14 6_53.25 Genome Set $18741 \mathrm{~N} / \mathrm{A}$ ICE Firmicutes

NZ_JH379341.1_Prevotella_stercorea_DSM_18206_genomic_scaffold_Scfld16_wh olēegenome_shotgun_sequence Genome Set $4918 \overline{2}$ N/A ICE Bacteroidetes

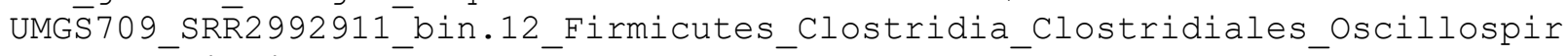

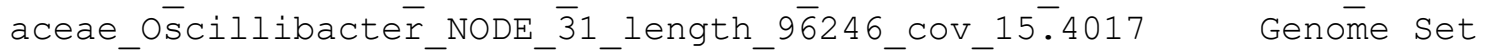

- 96246 N/A ICE Firmícutes 
21673_4_71_Firmicutes_Clostridia_Clostridiales_Peptostreptococcaceae_Clos tridium_.21673_471.8 Genome Set 133779 N/A ICE Firmicutes UMGS160 $\overline{7}$ SRR $60 \overline{5} 4 \overline{4} 90$ bin.8 Firmicutes Clostridia Clostridiales Lachnospira ceae_NA_NoDE_167_length_4 4405 cov_6.507892 Genome set $4440 \overline{5}$ N/A ICE

Firmicutes

UMGS2053_SRR2912776_bin.56_Firmicutes_Clostridia_Clostridiales_Peptostrep

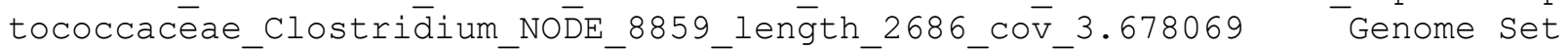
$2686 \overline{\mathrm{N}} / \mathrm{A}$ ICE $\overline{\mathrm{F}}$ irmicutes

NZ_CZPX01000005.1_Actinomyces_ihumii_strain_SD1_whole_genome_shotgun_seq ueñce Genome Set $\overline{1} 390721$ N/A N/A Actinobacteria UMGS120 ERR1018284 bin.6 Bacteroidetes Bacteroidia Bacteroidales Rikenell

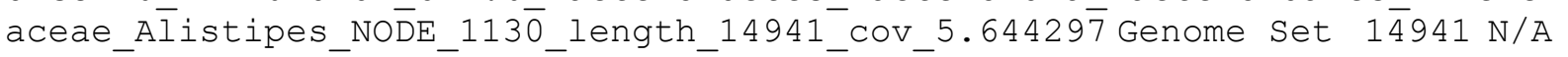
ICE Bacteroidetes

UMGS1236 ERR1293842 bin.23 Bacteroidetes Bacteroidia Bacteroidales Bacter oidaceae_Bacteroides_NODE_$\overline{7} 6$ _length_93040_cov_11.595053 Genome Set

$93 \overline{0} 40 \mathrm{~N} / \mathrm{A} \quad \mathrm{ICE}^{-}$Bacteroidetes

14207_6_3_Bacteroidetes_Bacteroidia_Bacteroidales_Porphyromonadaceae_NA_. $14207{ }^{-}{ }^{-} 3.25$ Genome Set $38355 \mathrm{~N} / \mathrm{A}$ ICE Bacteroidetes

NZ_KI $\overline{3} 9 \overline{1} 947.1$ Ruminococcaceae_bacterium_D16_genomic_scaffold_acsPy-

supercont2.1_whole_genome_shōtgun_sequence Genome Set $170 \overline{3} 898$ N/A

ICE Firmicutes

21673_4_87_Firmicutes_Clostridia_Clostridiales_Lachnospiraceae_Dorea_.216 73 4_87.5 Genome Set 213095 - N/A ICE Firmicutes

UMGGST̄735_ERR525802_bin.17_Firmicutes_Clostridia_Clostridiales_Ruminococca

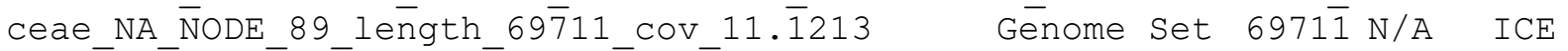

Firmicutes

UMGS1433_SRR6028647_bin.11_Bacteroidetes_Bacteroidia_Bacteroidales_Prevot

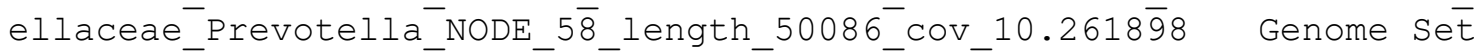

$50 \overline{0} 86 \mathrm{~N} / \mathrm{A} \quad \mathrm{ICE}$ Bäctēroidetés

21673_4_71_Firmicutes_Clostridia_Clostridiales_Peptostreptococcaceae_Clos

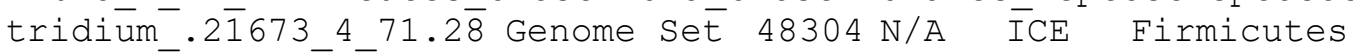

NZ_LT594620.1_Culturomica_massiliensis_strain_Marseille-

P2 $\overline{6} 98$ _whole_ḡenome_shotgün_sequence - Genomē set 173787 N/A ICE Bacteroidetes

NZ_JH376875.1_Clostridium_clostridioforme_2_1_49FAA_genomic_scaffold_supe rcōnt1.6_whole_genome_shōtgun_sequence Gēenomē set 276472 - N/A ICE Firmicutes

NZ_GL890521.1_Lachnospiraceae_bacterium_3_1_46FAA_genomic_scaffold_superc onṫ.2_whole_genome_shotgun_sequence Genome Set 919149 N/A ICE Firmicutes

14207_7_43_Firmicutes_Clostridia_Clostridiales_Lachnospiraceae_NA_.14207_ $743 . \overline{3}-{ }^{-}$- Genome Set $227190-$ N/A ICE Firmicutes

NZ_FCEY01000008.1_Clostridium_sp._AT5_genome_assembly_contig_contig00008 whole_genome_shōtgun_sequence - Genome Set 1822226 N/A ICE Firmicutes

NZ_JALB01000023.1_Providencia_alcalifaciens_RIMD_1656011_ctgN84L48809C36. $66 \overline{9} 4$ _whole_genomē_shotgun_sequence Genōme sēet 48809 N/A Plasmid Proteobactería

UMGS905_SRR6028570_bin.23_Firmicutes_Clostridia_Clostridiales_Ruminococca ceae_NA_NODE_129_lēength_4 2604 _cov_5. 810595 Genome Set $4260 \overline{4}$ N/A ICE Fírmicutes

UMGS59_ERR589527_bin.14_Firmicutes_Clostridia_Clostridiales_Eubacteriacea

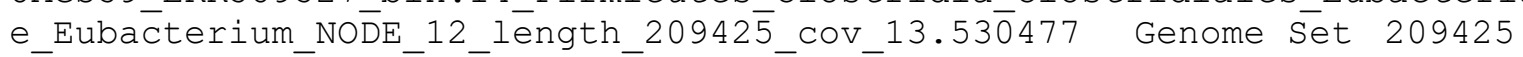
N/A ICE Fírmicutes 
UMGS1772_SRR341608_bin.22_Firmicutes_Clostridia_Clostridiales_Lachnospira ceae_NA_NODE_163_lēength_4 $\overline{8} 429 \_c o v \_1 . \overline{8} 19986$ Genome set $4842 \overline{9}$ N/A ICE

Firmicutes

NZ_KE150436.1_Coprococcus_sp._HPP0074_genomic_scaffold_acAqD-

supercont1.2_whole_genomé_shötgun_sequence Genome sēt 386540 N/A ICE Firmicutes

UMGS1206 ERR414305 bin.35 Firmicutes Clostridia Clostridiales NA NA NODE 1443 lenḡth 20391 Cov $11 . \overline{2} 02301$ Geñome set $2 \overline{0} 391 \mathrm{~N} / \mathrm{A}$ ICE Firmícutes NZ_DST 49678.1 Clostridium_scindens_ATCC_35704_Scfld_02_0_genomic_scaffold whole_genomé_shotgun_sequence $\bar{G}$ Firmicutes

14207_7_56_Firmicutes_Clostridia_Clostridiales_NA_NA_.14207_7_56.20 Genome set $6329 \overline{5}$ N/A ICE ${ }^{-}$Firmicutes

20287_6_63_Firmicutes_Clostridia_Clostridiales_Lachnospiraceae_NA_.20287_ $663 . \overline{2} 5^{-}$Genome Set $^{-} 13328 \mathrm{~N} / \mathrm{A}^{-}$ICE Firmicütes

UMGGS1680_SRR5558297_bin.55_Actinobacteria_Actinobacteria_Coriobacteriales _Coriobacteriaceae_Collinsēlla_NODE_854_lēength_35967_cov_10.287545

Genome set $3 \overline{5} 967$ N/A ICE ACtinobacteria

14207_7_95_Firmicutes_Clostridia_Clostridiales_Ruminococcaceae_NA_.14207_

7 95. ${ }^{-}$- Genome Set 221254 N/A ICE Firmicutes

200287_6_53_Bacteroidetes_Bacteroidia_Bacteroidales_Bacteroidaceae_Bactero ides_.20̄287_6_53.11 Gēnome set $28 \overline{1} 564 \quad$ N/A ICE Bacteroidetes UMGST̄995_SRR̄3108064_bin.38_Firmicutes_Clostridia_Clostridiales_Peptostrep

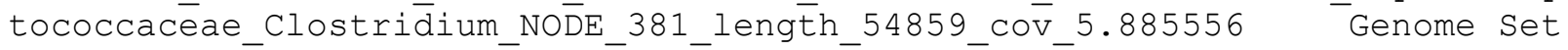
$54859 \overline{\mathrm{N}} / \mathrm{A} \quad \mathrm{N} / \mathrm{A} \quad \overline{\mathrm{F}} \mathrm{irmi} \overline{\mathrm{c} u t e} \overline{\mathrm{s}}$

NZ_KE150017.1_Lachnospiraceae_bacterium_2_1_46FAA_genomic_scaffold_aclXTsupercont-comp̄lete_whole_genome_shotgun_sequence Genome set $221 \overline{9} 029$

N/A ICE Firmicutes

UMGS413_ERR1305894_bin.87_Firmicutes_Clostridia_Clostridiales_Lachnospira ceae_NA_NODE_145_lēength_98533_Cov_19.400262 Genome set $9853 \overline{3}$ N/A ICE Fírmicütes

UMGS400_ERR414279_bin.29_Bacteroidetes_Bacteroidia_Bacteroidales_Porphyro monadacēae_NA_NODE_878_lēength_28135_cov ICE Bacteroidetes

UMGS1369_SRR5050586_bin.6_Firmicutes_Clostridia_Clostridiales_Lachnospira

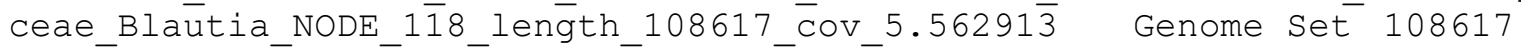

N/A ICE Firmicutes

12718_7_84_Firmicutes_Clostridia_Clostridiales_Ruminococcaceae_NA_.12718_

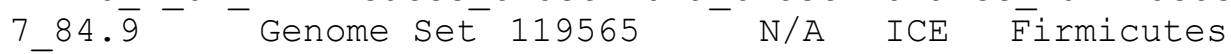

UM̄GS480_ERR011241_bin.5_Bacteroidetes_Bacteroidia_Bacteroidales_Rikenella

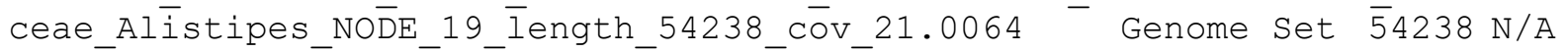

ICE Bacteroidetes

14207_7_70_Bacteroidetes_Bacteroidia_Bacteroidales_Rikenellaceae_Alistipe

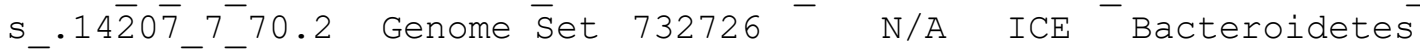

13414_6_33_Firmicutes_Clostridia_Clostridiales_Lachnospiraceae_NA_.13414_ $633 . \overline{6}-$ Genome set $244910-$ N/A ICE Firmicutes

13470_2_62_Bacteroidetes_Bacteroidia_Bacteroidales_Bacteroidaceae_Bactero ides_.13470_2_62.4 Genome set $25 \overline{3} 346$ N/A ICE Bacteroidetes 13414 6_15_Firmicutes_Clostridia_Clostridiales_Lachnospiraceae_NA_.13414_ $615 . \overline{3}{ }^{-}{ }^{-}$Genome Set 28176 N/A ICE Firmicutes

18048_2_90_Bacteroidetes_Bacteroidia_Bacteroidales_Porphyromonadaceae_odo ribacter_.1.18048_2_90.18- Genome Set 80499 N/A - ICE Bacteroidetes 
UMGS1017_ERR525737_bin.30_Tenericutes_Mollicutes_Erysipelotrichales_Erysi

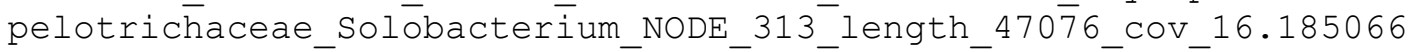

Genome Sét 47076 N/A ICE Tenericutes

14207770 Bacteroidetes Bacteroidia Bacteroidales Rikenellaceae Alistipe

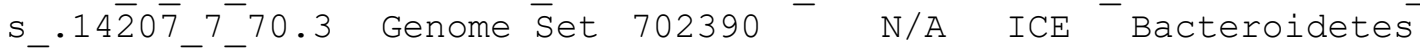

14207_7_18_Firmicutes_Clostridia_Clostridiales_NA_NA_.14207_7_18.1

Genome set $6645 \overline{3} 2 \quad$ N/A ICE Firmicutes

14207_7_23_Bacteroidetes_Bacteroidia_Bacteroidales_Bacteroidaceae_Bactero ides_. $1 \overline{4} 20 \overline{7} 7$ 7_23.46 Génome set $30 \overline{4} 11 \mathrm{~N} / \mathrm{A}$ ICE Bacteroidetes UMGST̄353_ERR $\overline{8} \overline{8} 9584$ bin.12_Firmicutes_Clostridia_Clostridiales_Lachnospira ceae_NA_NoDE_737_lēength_24663_cov_3. 31502 Genome set $2466 \overline{3}$ N/A ICE

Fírmicutes

UMGS1155_ERR209170_bin.2_Bacteroidetes_Bacteroidia_Bacteroidales_Prevotel laceae_Prevotella_N NODE_4 $\overline{4} 9$ _length_9462_Cov_9.844584 Genome Set $9 \overline{4} 62$ N/A $\overline{\mathrm{N}} / \mathrm{A} \quad$ Bacteroidetes

NZ_HE978610.1_Brevibacterium_sp._JC43_genomic_scaffold_scaffold00011_wh olēegenome_shōtgun_sequence Genome set $86061 \overline{\mathrm{N}} / \mathrm{A}$ ICE Actinobacteria 1341̄4_6_47_Firmicutes_Clostridia_Clostridiales_Lachnospiraceae_Lachnobact erium_.1341446_47.19-Genome Set $51925 \mathrm{~N} / \mathrm{A}$ ICE Firmicutes

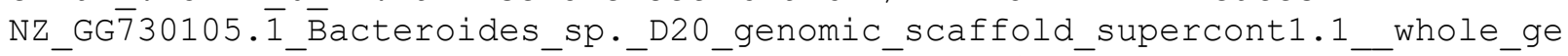
nome_shotgun_sequence Geñome Set $1436640^{-}$N/A ${ }^{-}$ICE Bacteroidetes NZ_EQ $973215 . \overline{1}$ Bacteroides_fragilis_3_1_12_supercont1.3_genomic_scaffold whōle_genome_shotgun_sequence_ Genome Set 707880 N/A ICE Bacteroidetes

UMGS2048 SRR1197080 bin.23 Tenericutes Mollicutes Erysipelotrichales Erys

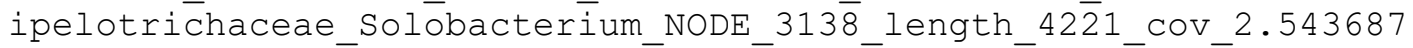

Genome Set 4221 N/A $\overline{\text { ICE }}$ Tenericutes

20287_6_37_Firmicutes_Clostridia_Clostridiales_NA_NA_.20287_6_37.37

Genome set $2538 \overline{3}$ N/A ICE ${ }^{-}$Firmicutes

14207_7_28_Firmicutes_Clostridia_Clostridiales_NA_NA_.14207_7_28.4 Genome set $5989 \overline{6} 3$ N/A ICE Firmicutes

UMGS1678_ERR589505_bin.72_Firmicutes_Negativicutes_Selenomonadales_Veillo

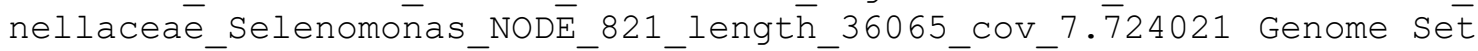
$360 \overline{65} \mathrm{~N} / \mathrm{A} \quad \mathrm{N} / \mathrm{A}-\mathrm{Firmicutes}$

UMGS96_SRR769526_bin.2_Firmicutes_Clostridia_Clostridiales_Lachnospiracea

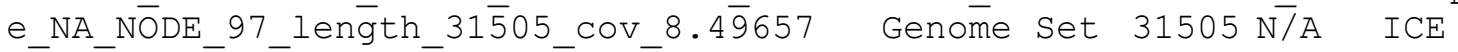

$$
\text { Firmicutes }
$$

13470_2_64_Bacteroidetes_Bacteroidia_Bacteroidales_Bacteroidaceae_Bactero ides_.13470_2_64.3 Genome Set $18 \overline{3} 539$ N/A ICE Bacteroidetes UMGS $\overline{6} 29$ SRR $\overline{5} 5 \overline{8} 0103$ bin.48_Actinobacteria_Actinobacteria_Coriobacteriales_ Coriobacteriaceae_NA_NODE_305_length_8437̄1_cov_8.804328 Genome Set $84371 \mathrm{~N} / \mathrm{A}$ ICE Actinob̄acteriā

UMGS151_ERR525714_bin.15_Firmicutes_Clostridia_Clostridiales_Lachnospirac

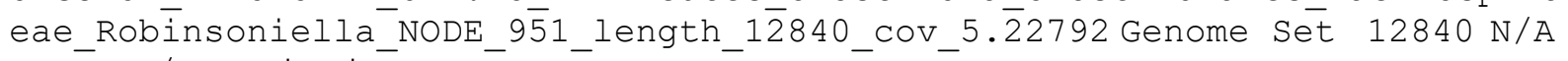
N/A Firmicutes

UMGS624_ERR011095_bin.1_Bacteroidetes_Bacteroidia_Bacteroidales_Bacteroid

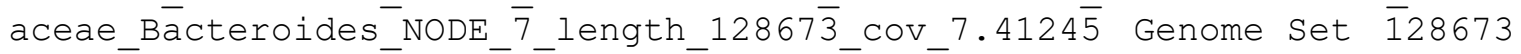

N/A ICE Bacterōidetes

UMGS21_ERR525840_bin.18_Firmicutes_Clostridia_Clostridiales_NA_NA_NODE_79 length_114292_cov_33.47 24 Genome Set $11429 \overline{2}$ N/A ICE Firmicutes UMGS511_DRR0423̄16_bin.21_Actinobacteria_Actinobacteria_Coriobacteriales_C oriobacteriaceae_Collinsēella_NODE_25_leñgth_186654_cov_10.933125

Genome set $186654 \quad \bar{N} / A{ }_{I C E}$ Actinobactēeria 
UMGS1236_ERR1293842_bin.23_Bacteroidetes_Bacteroidia_Bacteroidales_Bacter

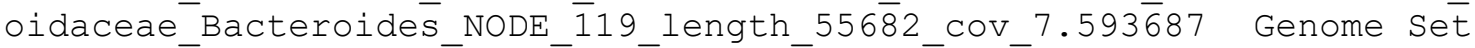
$55 \overline{6} 82 \mathrm{~N} / \mathrm{A}$ ICE Bacterōidetes

20298_3_65_Firmicutes_Clostridia_Clostridiales_Lachnospiraceae_NA_.20298_ $365 . \overline{3}-$ Genome Set $284648-$ N/A ICE Firmicutes

18048_1_70_Firmicutes_Clostridia_Clostridiales_Ruminococcaceae_NA_.18048_ $170 . \overline{4}-$ Genome Set $258203-$ N/A ICE Firmicutes

80080_1_80_Tenericutes_Mollicutes_Erysipelotrichales_Erysipelotrichaceae_S olobācterium_ERS138178.8080_1_80.11 Genome Set $\overline{1} 15840 \quad$ N/A ICE

Tenericutes

UMGS397_ERR1190862_bin.42_Actinobacteria_Actinobacteria_Coriobacteriales_ Coriobacteriaceae_Collinsēlla_NODE_8_lenḡth_245134_cov_ī2.823269

Genome set $\overline{2} 45134 \quad \bar{N} / A \quad \bar{I} \overline{C E}$ Actinobactēria

12718_7_84_Firmicutes_Clostridia_Clostridiales_Ruminococcaceae_NA_.12718_ $784 . \overline{4}-$ Genome Set 304438 N/A ICE Firmicutes

UMGGS923_ERR2013566_bin.18_Firmicutes_Clostridia_Clostridiales_Ruminococca

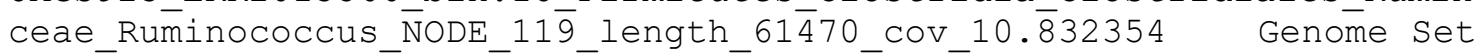

$61470 \mathrm{~N} / \mathrm{A}$ ICE Firmicutes

20298_3_39_Firmicutes_Clostridia_Clostridiales_NA_NA_.20298_3_39.7

Genome set $2131 \overline{3} 8 \quad$ N/A ${ }^{-}$ICE Firmicutes

UMGS1369_SRR5050586_bin.6_Firmicutes_Clostridia_Clostridiales_Lachnospira ceae_Blautia_NODE_39̄4lenḡth_34234_cov_5.781562- Genome set 34234 N/A

N/A Firmicutes

NZ_HE610718.1_Peptoniphilus_senegalensis_JC140_type_strain_JC140T_genom ic_scaffold_scaffold00002_whole_genome_shotgun_sequenceGeñome set
815711
$\mathrm{N} / \mathrm{A} \quad \mathrm{ICE}$
Firmicutes

UMGS622_ERR321346_bin.18_Bacteroidetes_Bacteroidia_Bacteroidales_Bacteroi

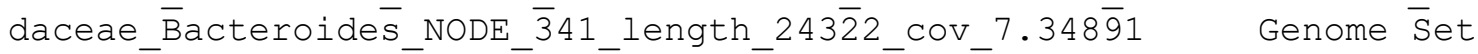

$24322 \mathrm{~N} / \mathrm{A} \quad \mathrm{N} / \mathrm{A}$ Bacteroidetés

UMGS365_SRR5558121_bin.15_Firmicutes_Clostridia_Clostridiales_NA_NA_NODE_ 53_length_151448_Cov_7.017̄811 Genome Set $1 \overline{51448}$ N/A I $\overline{C E}$

Firmicutes

UMGS1747_SRR3917697_bin.34_Actinobacteria_Actinobacteria_Coriobacteriales _Coriobacteriaceae_Collinsēlla_NODE_35_length_115142_cov_52.3374

Genome set $1 \overline{1} 5142 \quad \mathrm{~N} / \overline{\mathrm{A}} \quad \overline{\mathrm{IC}} \overline{\mathrm{C}}^{-}$Actinōbacterīa

18048_1_72_Firmicutes_Clostridia_Clostridiales_Lachnospiraceae_Blautia_.1 $8048 \overline{1} \overline{7} 2 . \overline{4}$ Genome Set 307071 - N/A ICE Firmicutes

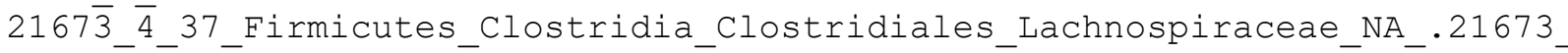
4_37.12 ${ }^{-}$Genome Set 92754 N/A ${ }^{-}$ICE Firmicütes

UMGGS708_SRR2155372_bin.20_Firmicutes_Clostridia_Clostridiales_NA_NA_NODE_ 85_length_95213_cov_14.6286 Genome set 95213 N/A ICE Firmicutes 180 $\overline{0} 8$ 1_7 $\overline{2}$ Firmicutēs_Clostridia_Clostridiales_Lachnospiraceae_Blautia_.1 $8048 \overline{1} \overline{7} 2 . \overline{1} 1$ Genome set $341 \overline{31}$ N/A N/A Firmicutes

UMGS $\overline{6} 2 \overline{9}$ _SRR5580103_bin.48_Actinobacteria_Actinobacteria_Coriobacteriales_

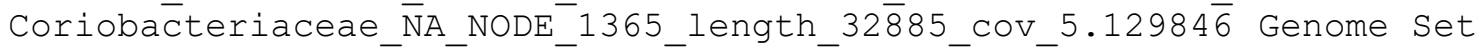

$32885 \mathrm{~N} / \mathrm{A} \quad \overline{\mathrm{N}} / \mathrm{A}$ Actinobācteria

13414_6_67_Firmicutes_Clostridia_Clostridiales_Lachnospiraceae_NA_.13414_ 6_67.14- Genome Set 105232 N/A ICE Firmicutes

UMGGS354_SRR4444801_bin.32 Firmicutes_Clostridia_Clostridiales Lachnospira ceae_NA_NODE_4_lenğth_316779_COV_26.9̄29188 Genome set $3167 \overline{7} 9$ N/A ICE Firmicutes

UMGS607_ERR526073_bin.39_Bacteroidetes_Flavobacteriia_Flavobacteriales_Fl avobactēeriaceae_F̄̄avobacterium_NODE_355_length_36269_cov_4.121804

Genome set 36269 N/A I $\overline{C E}$ Bacteroidetes 
NZ_KQ236098.1_Parabacteroides_sp._2_1_7_genomic_scaffold_supercont2.7_wh olē_genome_shōtgun_sequence Genome Set 146439

rep_cluster_1702, rep_cluster_1097 ICE Bacteroidetes

14207_6_9_Bacteroìidetes_BacteroidiāBacteroidales_Bacteroidaceae_Bacteroi des_. $\overline{1} 4 \overline{2} 0 \overline{7}$ 6_9.4 Genome Set $176550^{-}$N/A ICE Bacteroidetes

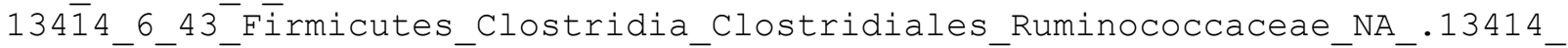
$643 . \overline{1}-$ Genome Set $1011832-$ N/A ICE Firmicutes

NZ__GG705177.1_Bacteroides_sp._2_1_22_genomic_scaffold_supercont1.5_whole _genome_shotgünsequence ${ }^{-} \overline{\text { Genome Set }} 4140 \overline{6} 1$ N/A ICE

Bàcteroidetes

NZ_DS996922.1_[Bacteroides]_pectinophilus_ATCC_43243_Scfld_02_2_genomic_s caffold_wholè_genome_shotgun_sequence Genome $\overline{-}^{-}$Set $\overline{11} 2627^{-}-\overline{\mathrm{N}} / \mathrm{A}$ ICE Firmicutes

13414_6_68_Firmicutes_Clostridia_Clostridiales_Lachnospiraceae_NA_.13414_ $668 . \overline{3}^{-}$- Genome Set $348365-$ N/A ICE Firmicutes

UM̄GS597_ERR1305903_bin.25_Firmicutes_Clostridia_Clostridiales_NA_NA_NODE_ 124_lenḡth_92902_Cov_15.1̄13832 Genome Set $9 \overline{2} 902$ N/A N/A Firmícutes 21673_4_12_Firmicutes_Clostridia_Clostridiales_Lachnospiraceae_NA_.21673_

$412 . \overline{7}-$ Genome Set $221672-$ N/A ICE Firmicutes

NZ̄_GG730320.1_Clostridium_sp._M62_1_genomic_scaffold_Scfld11_whole_genom e_shotgun_sequence Geñome Set $30658 \mathrm{~N} / \mathrm{A}^{-}$ICE Firmicutes

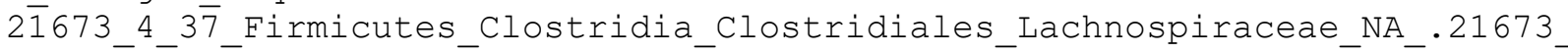
4_37.13 - Genome Set $80682 \mathrm{~N} / \mathrm{A}^{-}$ICE Firmicütes

UMMGS1293_SRR5558313_bin.90_Actinobacteria_Actinobacteria_Coriobacteriales _Coriobacteriaceae_N

N/A ICE Actinobacteria

NZ_CCXH01000302.1_Actinomyces_polynesiensis_strain_MS2_whole_genome_shot guñ_sequence Genome Set $32881 \mathrm{rep}$ cluster_1578 N/A Actinobacteria NZ_JH379375.1_Prevotella_stercorea_DSM_18206_genomic_scaffold_Scfld115_w hole_genome_shotgun_sequence Genome Set $1066 \overline{2} 8$ N/A ICE

Bacteroidetes

NZ_LN831785.1_Massilibacterium_senegalense_strain_mt8_whole_genome_shotg un_sequence Genome set $521830^{-}$N/A ICE Firmicutes

UMGGS1236_ERR1293842_bin.23_Bacteroidetes_Bacteroidia_Bacteroidales_Bacter oidaceae_Bacteroides_NODE_439_length_16698_cov_9.520940 Genome Set

$16 \overline{6} 98 \mathrm{~N} / \mathrm{A} \quad \mathrm{ICE}^{-}$Bacterōidetes

UMGS1294_ERR414346_bin.49_Bacteroidetes_Bacteroidia_Bacteroidales_Prevote liaceae_Prevotella_NODE_3891_length_10009_Cov_3.919630 Genome Set

$1 \overline{0009} \mathrm{~N} / \mathrm{A} \quad \mathrm{IC} E$ Bacteroidetes

UMGS 840 SRR3737012_bin.13_Bacteroidetes_Bacteroidia_Bacteroidales_Rikenel laceae_Ālistipes_NŌDE_94_İength_87111_COV_6.572907 Genome Set $87 \overline{1} 11$ N/A

$\overline{\mathrm{I}} \mathrm{E}$ Bacteroidetes

NZ_GG663520.1_Butyrivibrio_crossotus_DSM_2876_genomic_scaffold_Scfld16_W hole_genome_shotgun_sequence Genome set $\overline{3} 0575 \overline{5}$ N/A ICE Firmicutes UMGS1236_ERR1293842_bin.23_Bacteroidetes_Bacteroidia_Bacteroidales_Bacter

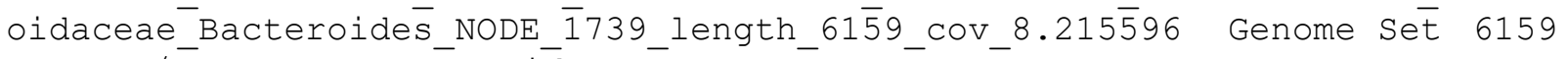

$\mathrm{N} / \overline{\mathrm{A}}$ ICE Bacteroidetes

UMGS553_ERR209769_bin.23_Firmicutes_Clostridia_Clostridiales_Ruminococcac eae_NA_NODE_160_length_58516_COV_3.773781 Genome Set $585 \overline{1} 6$ N/A ICE Firmicutes

UMGS639_DRR042328_bin.21_Actinobacteria_Actinobacteria_Coriobacteriales_C oriobacteriaceae_Collinsēlla_NoDE_2_length_962117_cov_̄̄ 5.533915

Genome set $962117 \quad$ N/A ${ }^{-}$CE Actinobacteriā 
UMGS1204_SRR1761701_bin.34_Actinobacteria_Actinobacteria_Coriobacteriales

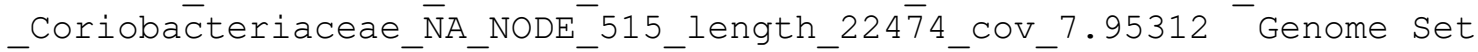

$22474 \mathrm{~N} / \mathrm{A} \quad \mathrm{IC} E-$ Actinobācteria

8080178 Firmicutes Clostridia Clostridiales Peptostreptococcaceae Clost ridium_ERS̄138176.8080_1_78.27 - Genome set 94624 N/A ICE Firmicutes UMGS361_SRR5056748_bin. $\overline{6} 3$ _Firmicutes_Clostridia_Clostridiales_NA_NA_NODE_ 1551 length $29861 \overline{\mathrm{COV}} 7.8 \overline{8} 0427$ Geñome set $2 \overline{9} 861 \mathrm{~N} / \mathrm{A}$ ICE - Fírmícutes UMGST̄314_ERR̄ $1195 \overline{8}$ bin̄.26_Firmicutes_Clostridia_Clostridiales_Peptostrept ococcaceāe_Clostrī̄ium_NOD̄E_2996_lenḡth_13635_cov_167.319072 - Genome Set $1363 \overline{5} \mathrm{~N} / \mathrm{A}$ Plasmid Firmicutes

14207_6_9_Bacteroidetes_Bacteroidia_Bacteroidales_Bacteroidaceae_Bacteroi des_. $\overline{1} 4 \overline{2} 0 \overline{7} 6$ 6.46Genome Set $28505 \mathrm{~N} / \mathrm{A}$ ICE Bacteroidetes

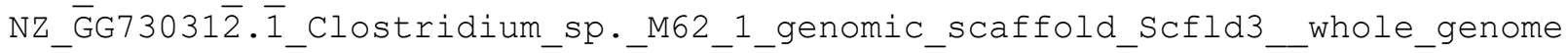
shotgun sequēenceGenome Sét $\overline{412181}{ }^{-}$N/A ${ }^{-}$ICE Firmicutes

$\bar{N} Z$ _DS990130.1_Bacteroides_plebeius_DSM_17135_Scfld_02_13_genomic_scaffold whole_genomésshotgun_sequence $\bar{G}$ Genome Set $7602 \overline{60}{ }^{-}{ }^{-}$N/A I $\bar{C} E$

Bacteroidetes

14207_7_37_Firmicutes_Clostridia_Clostridiales_Lachnospiraceae_Dorea_.142 077 37.7 - Genome Set 156954 N/A ICE Firmicutes

$21 \overline{6} 7 \overline{3}$ _4_77_Firmicutes_Clostridia_Clostridiales_Peptostreptococcaceae_Clos tridium-.21673477.2 Genome SeE 1004140 N/A ICE Firmicutes

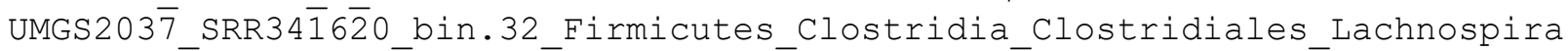

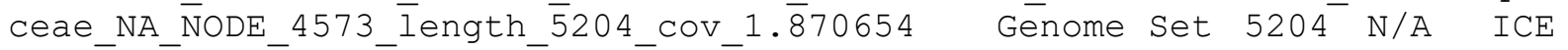

Firmicutes

UMGS29 ERR525845 bin.24 Bacteroidetes Bacteroidia Bacteroidales Porphyrom onadacēae_Butyricimonas_NODE_1_length_695009_cov_28.956 Genome Set
$695 \overline{009}$
N/A ICE Bacteroidetes

UMGS376_DRR042411 bin.15_Actinobacteria_Actinobacteria_Coriobacteriales_C

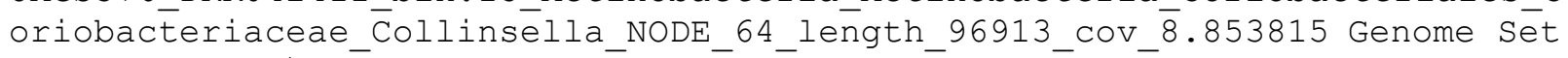
$96913 \mathrm{~N} / \mathrm{A}$ ICE Actinōbactēriā

NZ_HE610960.1_Paenibacillus_senegalensis_JC66_genomic_scaffold_scaffold0 $00 \overline{0} 4$ _whole_genome_shotgun_sequence Genomé set 601914 $\bar{N} / A \quad$ ICE Firmicutes

14207_7_90_Firmicutes_Clostridia_Clostridiales_NA_NA_.14207_7_90.30 Genome Set $3974 \overline{3}$ N/A ICE Firmicutes

13414_6_15_Firmicutes_Clostridia_Clostridiales_Lachnospiraceae_NA_.13414_ $615 . \overline{2}-$ Genome set $268840-$ N/A ICE Firmicutes

144207_6_13_Bacteroidetes_Bacteroidia_Bacteroidales_Bacteroidaceae_Bactero ides_.14207_6_13.7 Genome Set 128978 N/A ICE Bacteroidetes UMGST̄049_ERR $\bar{R} \overline{2} 1543$ bin.17_Bacteroidetes_Bacteroidia_Bacteroidales_Porphyr

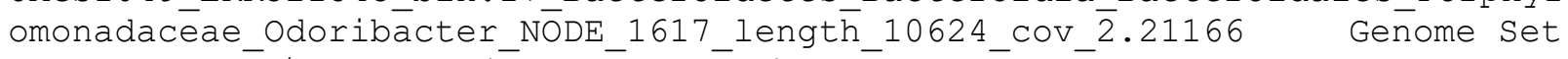
$10624 \overline{\mathrm{N}} / \mathrm{A} \quad$ Plasmid $\overline{\mathrm{d}}$ Bacteroidetes

UMGS1765_ERR525886_bin.15_Fusobacteria_Fusobacteriia_Fusobacteriales_Fuso bacteriac̄eae_Fusobācterium_NODE_920_length_21923_cov_18.618118 Genomē Set $21923 \mathrm{~N} / \mathrm{A}$ ICE Fusōbactēria

18048_2_82_Firmicutes_Clostridia_Clostridiales_NA_NA_.18048_2_82.3 Genome set $1459 \overline{3} 1 \quad$ N/A ICE Firmicutes

NZ_LT608321.1_Prevotellaceae_bacterium_MarseilleP2826_whole_genome_shotgun_sequence_Genome set 2762878 N/A ICE UMGS1353_ERR589584_bin.12_Firmicutes_Clostridia_Clostridiales_Lachnospira

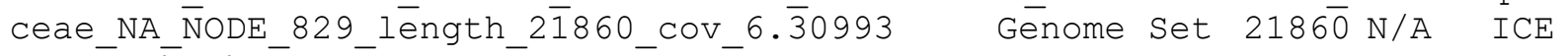
Firmicutes 
NZ_GL892011.1_Dysgonomonas_mossii_DSM_22836_genomic_scaffold_supercont1.8 whole_genomés shotgun_sequence Genome Set $17978 \overline{9} \quad$ N/A ${ }^{-}$ICE

Bäcteroidetes

13414666 Firmicutes Clostridia Clostridiales Lachnospiraceae Blautia .1 $3414 \overline{6} \overline{6} 6 . \overline{9}$ Genome Set $148432-$ N/A ICE Firmicutes

UMGS $\overline{1} 5 \overline{6}$ SRR5558393 bin. 8 Bacteroidetes Bacteroidia Bacteroidales Prevotel laceae_Prevotella_NoDE_9 $\overline{4}$ _length_74079_COV_8.40755 $\overline{7}$ Genome Set $7 \overline{4} 079$ N/A

ICE Bacteroidetes

21673_4_80_Firmicutes_Clostridia_Clostridiales_Peptostreptococcaceae_Clos tridium_.21673_4_80.1- Genome Set $1120401 \quad \overline{\mathrm{N}} / \mathrm{A} \quad$ ICE Firmicutes

20298_3_48_Firmicutes_Clostridia_Clostridiales_Lachnospiraceae_Blautia_.2 $0298 \overline{3} \overline{4} 8 . \overline{3} 4 \quad$ Genome set $187 \overline{73}$ N/A ICE Firmicutes

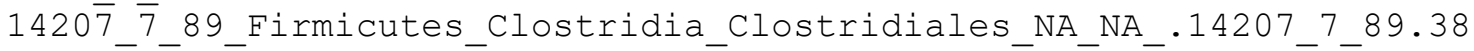

Genome Set $3358 \overline{7}$ N/A ICE ${ }^{-}$Firmicutes

UMGS1953 SRR5127782 bin.22 Firmicutes Clostridia Clostridiales NA NA NODE 657_length_9956_cov_5.000404 Genome set 9956 N/A ICE Firmicutes $\bar{N} Z$ GL538348.1_Faécalïbacterium_cf._prausnitzii_KLE1255_genomic_scaffold_S cfīd702_wholè_genome_shotgun_sequence Genome- Set 12446 N/A ICE

Firmicutes

13414_6_68_Firmicutes_Clostridia_Clostridiales_Lachnospiraceae_NA_.13414 $668 . \overline{8}-$ Genome Set 200861 - N/A ICE Firmicutes

UM̄GS1126_ERR414269_bin.20_Bacteroidetes_Bacteroidia_Bacteroidales_Porphyr omonadacēae_NA_NODE_4054_īength_6532_coV_7.024240 Genome set $65 \overline{3} 2$ N/A ICE Bacteroidetes

14207_6_3_Bacteroidetes_Bacteroidia_Bacteroidales_Porphyromonadaceae_NA_. $14207^{-}{ }^{-}{ }^{-} .7$ Genome set $\overline{1} 35988$ N/A ICE Bacteroidetes UMGS762_SRR5127675_bin.13_Firmicutes_Clostridia_Clostridiales_NA_NA_NODE_

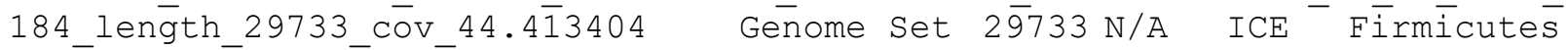
134144_6_68_Firmicutes_Clostridia_Clostridiales_Lachnospiraceae_NA_.13414_ 6_68.4 - Genome Set $292661-$ N/A ICE Firmicutes

200298_2_23_Bacteroidetes_Bacteroidia_Bacteroidales_Bacteroidaceae_Bactero ides_.2029 1341 $\overline{4}$ 6_10_Teñericutes_Mollicutes_Erysipelotrichales_Erysipelotrichaceae_ NA_.13 $4 \overline{1} 4 \overline{6}_{1} 10.13$ Genome Set $5215 \overline{7}$ N/A ICE Tenericutes

UMGGS320_SRRT5279258_bin.3_Actinobacteria_Actinobacteria_Coriobacteriales_C oriobact̄eriaceae_Cōllinsēlla_NODE_7_lenḡth_208815_cov_1̄3.470188

Genome Set $208815 \quad$ N/A ICE Actinobacteriā

20287670 Actinobacteria Actinobacteria Coriobacteriales Coriobacteriace ae NA_-. $202 \overline{8} 7$ 6_70.2 Geñome Set $270850^{-}$N/A ICE Āctinobacteria 21̄̄73_4_33_Bācteroidetes_Bacteroidia_Bacteroidales_Porphyromonadaceae_Par abactēeröidēs_.21673_4_33.11 Genome set 203470 rep_cluster_663 ICE

Bacteroidetes

12718_7_51_Firmicutes_Clostridia_Clostridiales_Lachnospiraceae_Ruminococc

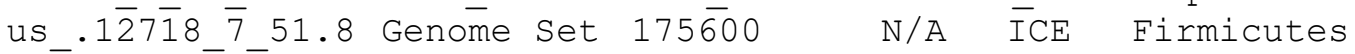

UMGGS505_ERRT1293707_bin.3_Firmicutes_Clostridia_Clostridiales_Ruminococcac eae_Ruminococcus_NŌDE_52_length_86150_Cov_4.2706666 Genome Sēt 86150 N/A ICE Firmicutes

NZ_DS499577.1_Alistipes_putredinis_DSM_17216_Scfld_02_7_genomic_scaffold _whole_genome_shotgun_sequence Genome Set $1197 \overline{6} 41^{-}$- rep_clüster_170 $\overline{2}$ ICE Bacteroidetes

13414_6_66_Firmicutes_Clostridia_Clostridiales_Lachnospiraceae_Blautia_.1 $3414 \overline{6} \overline{6} 6 . \overline{6}$ Genome Set $193764-$ N/A ICE Firmicutes 12718_7_72_Firmicutes_Clostridia_Clostridiales_NA_NA_.12718_7_72.4 Genome Set $2705 \overline{4} 1 \quad$ N/A ${ }^{-}$ICE Firmicutes 
NZ_KE993572.1_Clostridium_sp._ATCC_29733_genomic_scaffold_Scaffold222_wh olè genome shōtgun sequence Genome set $\overline{6} 8276 \mathrm{~N} / \overline{\mathrm{A}}$ ICE $\overline{\mathrm{F}}$ irmicutes UMGS̄ 1549 STRR1761710_bin.1_Actinobacteria_Actinobacteria_Coriobacteriales_ Coriobacteriaceae Cōllinsēlla NODE 394 lēength 28317 cov 2.395549

Genome set 28317 N/A ICE $\bar{A} c t i n o b a c t e r i a$

UMGS1348_ERR1305901_bin.5_NA_NA_NA_NA_NA_NODE_537_length_74508_COV_6.2132 49 Geñome set $74 \overline{5} 08 \mathrm{~N} / \overline{\mathrm{A}}$ ICE $\overline{\mathrm{N} A}$

13414_6_53_Firmicutes_Clostridia_Clostridiales_Ruminococcaceae_Faecalibac

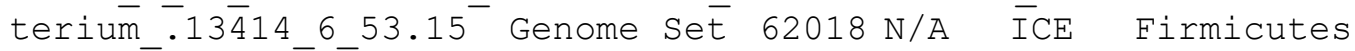

20298_3_39_Firmicutes_Clostridia_Clostridiales_NA_NA_.20298_3_39.12

Genome Set $1225 \overline{3} 3 \quad$ N/A ${ }^{-}$ICE Firmicutes

UMGS947_SRR1196456_bin.17_Firmicutes_Clostridia_Clostridiales_NA_NA_NODE

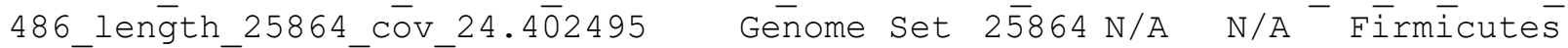
UMG $\bar{S} 881$ SRR $40330 \overline{7} 7$ binn.13 Firmicutes Clostridia Clostridiales Lachnospira ceae_Psēudobutyrivíbrio_NŌDE_5599_length_3069_cóv_15.672860 - Genome Set 3069 N/A N/A Firmicutes

14207_7_37_Firmicutes_Clostridia_Clostridiales_Lachnospiraceae_Dorea_.142 $077^{7} \overline{3} 7.3$ - Genome Set 187044 N/A ICE Firmicutes

UMGGST90_SRR5279243_bin.1_Actinobacteria_Actinobacteria_Coriobacteriales_C oriobact̄eriaceae_Cōllinsēlla_NODE_137_lēngth_85367_cov_6.370159

Genome Set 85367 N/A ICE Actinobacteria

UMGS761_SRR5056797_bin.113_Firmicutes_Clostridia_Clostridiales_RuminococC

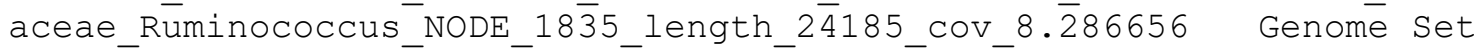

$24185 \mathrm{~N} / \mathrm{A} \quad \mathrm{IC} E$ Firmicutes

UMGS789_SRR5057091_bin.53_Actinobacteria_Actinobacteria_Coriobacteriales Coriobacteriaceae_Collinsēlla_NODE_77_length_95154_cov_六.227710

Genome Set $95154 \mathrm{~N} / \mathrm{A} \quad \overline{\mathrm{N}} / \mathrm{A} \quad \bar{A} c t \overline{i n o b a c t e} r i a$

NC_010371.1_Finegoldia_magna_ATCC_29328_plasmid_pFMC_DNA_complete_sequen $\mathrm{ce}^{-}$Genomé Set $18916 \overline{3}$ rep_cluster_1411 Plasmid $\bar{d}$ Firmicutes NZ_JH379347.1_Prevotella_stercorea_DSM_18206_genomic_scaffold_Scfld24_wh olēegenome_shōtgun_sequeñe Genome set 5911- N/A ICE Bactēeroidetes 20298_3_35_Firmicutes_Clostridia_Clostridiales_Lachnospiraceae_Blautia_.2 $0298 \overline{3} \overline{3} 5 . \overline{1}$ Genome Set 385819 - N/A ICE Firmicutes

14207_7_59_Firmicutes_Clostridia_Clostridiales_NA_NA_.14207_7_59.4 Genome set $2133 \overline{5} 1 \quad$ N/A ${ }^{-}$ICE Firmicütes

UMGS1711_SRR5558284_bin.37_Firmicutes_Clostridia_Clostridiales_Peptostrep

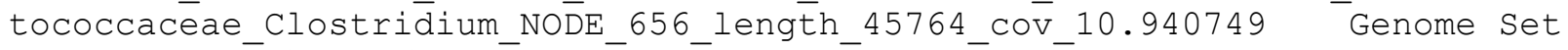
$45764 \overline{\mathrm{N}} / \mathrm{A} \quad \mathrm{N} / \mathrm{A} \quad \overline{\mathrm{F}} \mathrm{irmi} \overline{\mathrm{c} u t e} \overline{\mathrm{S}}$

UMGS1829_ERR209478_bin.33_Bacteroidetes_Bacteroidia_Bacteroidales_Porphyr omonadacēee_NA_NODE_1371_Iength_13317_Cov_2.051124 Genome Set $13 \overline{3} 17$ N/A ICE Bacteroidetes

UMGS503_SRR1196634_bin.19_Tenericutes_Mollicutes_Erysipelotrichales_Erysi pelotrichaceae_Solōbacterium_NODE_187_length_33267_cov_8.88155 Genome Set $33267 \mathrm{~N} / \mathrm{A}^{-} \quad \mathrm{N} / \mathrm{A} \quad$ Tenerícutes

20287_6_63_Firmicutes_Clostridia_Clostridiales_Lachnospiraceae_NA_.20287_ $663 . \overline{2}-{ }^{-}$- Genome Set 615097 - N/A ICE Firmicutes

13414_6_68_Firmicutes_Clostridia_Clostridiales_Lachnospiraceae_NA_.13414_ 6_68. ${ }^{-}-$Genome Set 158658 N/A ICE Firmicutes

200287_6_37_Firmicutes_Clostridia_Clostridiales_NA_NA_.20287_6_37.32

Genome set $3344 \overline{9}$ N/A N/A Firmicutes

UMGS1730_SRR5275432_bin.16_Firmicutes_Clostridia_Clostridiales_NA_NA_NODE _672_length_19595_cov_8.634596 Genome set $19 \overline{595}$ N/A ICE Firmicutes 
UMGS1423_ERR1018270_bin.44_Firmicutes_Clostridia_Clostridiales_Peptostrep

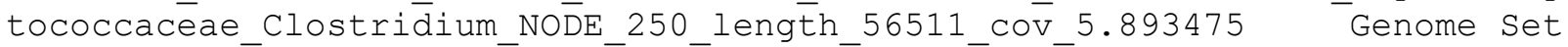
$56511 \overline{\mathrm{N}} / \mathrm{A} \quad \mathrm{N} / \mathrm{A} \quad \overline{\mathrm{F}}$ irmicutes

UMGS658 SRR2155387 bin.7 Firmicutes Clostridia Clostridiales NA NA NODE 3 6 length $\bar{h}_{155406 \text { cov }} 18.4 \overline{8} 47$ Genome Set $155406^{-}$N/A ICE ${ }^{-} \overline{\text { Firmicutes }}$ 18̄048_2_82_Firmícutēes_Clostridia_Clostridiales_NA_NA_.18048_2_82.14

Genome set $7337 \overline{3}$ N/A ICE ${ }^{-}$Firmicutes

NZ_LN913013.1_Gabonibacter_massiliensis_strain_GM7_whole_genome_shotgun sequence Genome set $232 \overline{4} 047$ N/A ICE Bacteroidetés 13470_2_93_Firmicutes_Clostridia_Clostridiales_Lachnospiraceae_NA_.13470_ 2_93. $\overline{3} 7^{-}$Genome Set $12214 \mathrm{~N} / \mathrm{A}^{-}$ICE Firmicütes

NZ̄_AAVN02000004.1_Collinsella_aerofaciens_ATCC_25986_C_aerofaciens$2 . \overline{0} \_$Cont973_wholè_genome_shotgun_sequence $\bar{e}$ Genome $\overline{\text { Set }} 184100$ N/A ICE Actinobacteria

18048_1_70_Firmicutes_Clostridia_Clostridiales_Ruminococcaceae_NA_.18048_ 1_70.1- Genome Set 602315 - N/A ICE Firmicutes

13̄14_6_33_Firmicutes_Clostridia_Clostridiales_Lachnospiraceae_NA_.13414_ $633 . \overline{1}-$ Genome Set $323356-$ N/A ICE Firmicutes

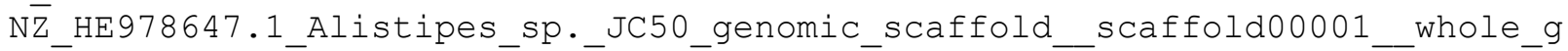

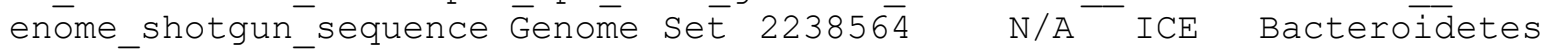
21673_4_12_Firmicutes_Clostridia_Clostridiales_Lachnospiraceae_NA_.21673 $412 . \overline{3} 6^{-}$Genome Set 8535 N/A ${ }^{-}$ICE Firmicutes

UMMGS664_ERR209886_bin.11_Firmicutes_Clostridia_Clostridiales_Ruminococcac eae_NA_NoDE_90_length_68815_cov_4.7444939 Genome set $688 \overline{1} 5$ N/A ICE

Firmicutes

NZ_KI271165.1_Eubacterium_ramulus_ATCC_29099_genomic_scaffold_Scaffold588 whole_genomé_shotgun_sequence Genome Set 1041 N/A N/A Firmicutes 20287_6_63_Firmicutes_C̄lostridia_Clostridiales_Lachnospiraceae_NA_.20287_ $663 . \overline{3}-$ Genome Set 307381 N/A ICE Firmicutes

UMMGS1389_ERR1620298_bin.4_Firmicutes_Clostridia_Clostridiales_NA_NA_NODE_ 3_length_440201_cov_21.639940 Genome set $4 \overline{40201}$ N/A I $\overline{C E}$

Firmicutes

20298_2_28_Firmicutes_Clostridia_Clostridiales_NA_NA_.20298_2_28.12

Genome set $7990 \overline{1}$ N/A ICE Firmicutes

20427_4_25_Firmicutes_Clostridia_Clostridiales_Lachnospiraceae_Lachnobact

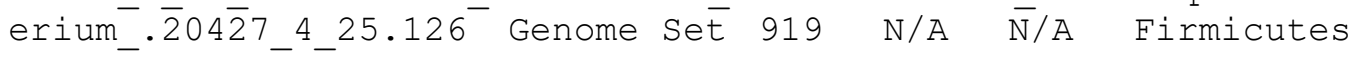

20298_2_23_Bäcteroidetes_Bacteroidia_Bacteroidales_Bacteroidaceae_Bactero ides_.20298_2_23.9 Genome Set $14 \overline{4} 167$ N/A ICE Bacteroídetes UMGST̈126_ERR $4 \overline{1} 4269$ bin.20_Bacteroidetes_Bacteroidia_Bacteroidales_Porphyr

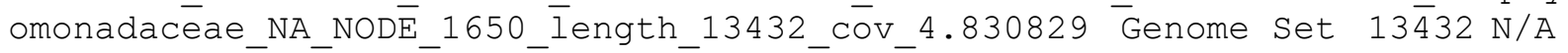
ICE Bacteroidetes

UMGS537_ERR1297716_bin.14_Firmicutes_Clostridia_Clostridiales_NA_NA_NODE_ 7_length̄h_192507_cov_11.219̄951 Genome set $1 \overline{92507}$ N/A N/A

Firmicutes

14207_7_56_Firmicutes_Clostridia_Clostridiales_NA_NA_.14207_7_56.33 Genome Set $1606 \overline{8}$ N/A N/A Firmicutes

UMGS1684_ERR011192_bin.15_Bacteroidetes_Bacteroidia_Bacteroidales_Prevote

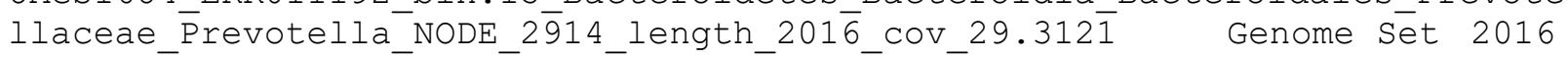
$\mathrm{N} / \bar{A} \quad$ ICE Bacteroidetés

14207_7_23_Bacteroidetes_Bacteroidia_Bacteroidales_Bacteroidaceae_Bactero ides_.14 $20 \overline{7}$ 7_23.44 Genome set $34 \overline{6} 96$ N/A ICE Bacteroidetes NZ_AD̄DR0200000 1.1 Desulfovibrio_sp._3_1_syn3_acqcXsupercont2.1_whole_genome_shotgun_sequence-Genome set 2716392 N/A ICE Proteobacteria 
NZ_HE998567.1_Holdemania_sp._AP2_genomic_scaffold_scaffold00001_whole_g enōme shotgun sequence Gēnomè SeEt $168508 \overline{9} \quad$ N/A ICE Tenericutes UMGS $9 \overline{4} 5$ ERR16 20269 bin. 39 Firmicutes Clostridia Clostridiales Peptostrept

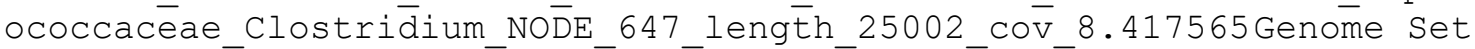
$2500 \overline{2} \mathrm{~N} / \mathrm{A} \quad \mathrm{ICE}$ Firmicutess

NZ_GL629647.1_Prevotella_salivae_DSM_15606_genomic_scaffold_SCAFFOLD1_wh olēe_genome_shōtgun_sequeñce Genome Set $31 \overline{8} 0194 \quad$ N/A ICE

Bacteroidetes

12718_7_47_Firmicutes_Clostridia_Clostridiales_NA_NA_.12718_7_47.4

Genome set $4607 \overline{1} 5 \quad$ N/A - ICE Firmicutes

UMGS1084_SRR4305433_bin.6_NA_NA_NA_NA_NA_NODE_136_length_49193_COV_12.121

474 Geñome set $49 \overline{1} 93 \mathrm{~N} / \overline{\mathrm{A}} \overline{\mathrm{ICE}} \overline{\mathrm{N}} \mathrm{A}$

11861 6_65_Firmicutes_Clostridia_Clostridiales_Ruminococcaceae_NA_.11861 $665 . \overline{4} 2^{-}$Genome Set 4391 N/A ${ }^{-}$ICE Firmicutes

UM̄GS1989 ERR1190931 bin.4 Firmicutes Clostridia Clostridiales NA NA NODE

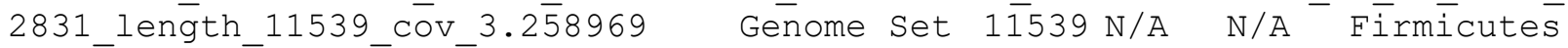

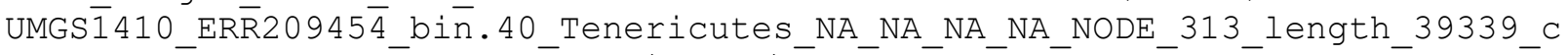
ov 7.704 $\overline{9} 43$ Genome Set $39 \overline{3} 39 \mathrm{~N} / \mathrm{A} \quad \mathrm{N} / \mathrm{A}^{-}$Tenericutés

UMḠGS1288_SRR341667_bin.16_Firmicutes_Clostridia_Clostridiales_Peptostrept

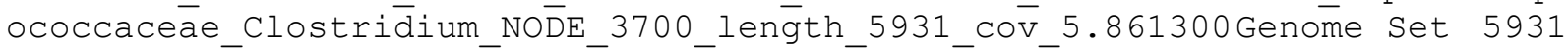

N/A ICE Firmicutes

UMGS305_SRR3582148_bin.9_Actinobacteria_Actinobacteria_Coriobacteriales_C oriobacteriaceae_Cöllinsēlla_NODE_34_leñgth_123690_cov_10.649816

Genome set $123690 \quad \bar{N} / A$ ICE Actinobactēria

UMGS119 ERR1620292 bin.17 Firmicutes Clostridia Clostridiales Lachnospira

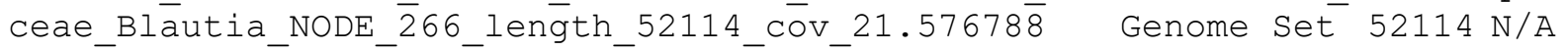

ICE Firmicutes

NZ_KQ236096.1_Parabacteroides_sp.2_1_7_genomic_scaffold_supercont2.5 wh olēegenome_shōtgun_sequence Genome Set $3038395^{-}{ }^{-}$N/A ICE

Bactēroidetes

UMGS1003_ERR525813_bin.62_Bacteroidetes_Bacteroidia_Bacteroidales_Prevote

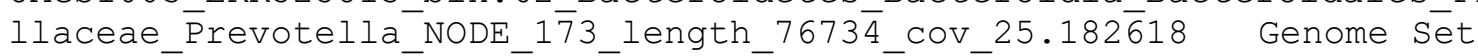

$7 \overline{6} 734 \mathrm{~N} / \mathrm{A} \quad \mathrm{N} / \mathrm{A} \quad \bar{B}$ actēroidetēs

NZ_KB905489.1_Bacteroides_sp._HPS0048_genomic_scaffold_acKdP-

supercont1.3_whole_genome_shötgun_sequence Genome Sēt 868688 N/A

ICE Bacteroidetes

UMGS2065_SRR2992944_bin.2_Tenericutes_NA_NA_NA_NA_NODE_75_length_13360_co

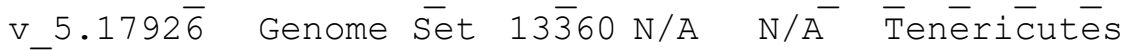

UM̄GS57_SRR2155361_bin.23_Firmicutes_Clostridia_Clostridiales_NA_NA_NODE_4 34 lenḡth_25917_cov_11.86̈8340 Genome set 25917 N/A ICE $\overline{\text { Firmicutes }}$

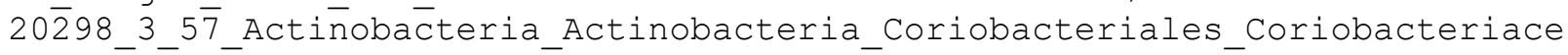

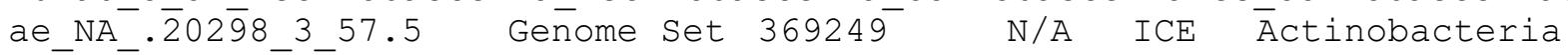
UMG S S 00 _ERR1 $29 \overline{3} 918$ bin.21_Bacteroidetes_Bacteroidia_Bacteroidales_Porphyr omonadāeae_NA_NODE_10_leñgth_171207_cov_21.154909 Genome Set $17 \overline{1} 207$

N/A ICE Bacteroidetes

13414 6_67_Firmicutes_Clostridia_Clostridiales_Lachnospiraceae_NA_.13414_ 6 67.53- Genome Set $886 \quad$ N/A ${ }^{-}$N/A Firmicūtes

NZ_FCEY01000007.1_Clostridium_sp._AT5_genome_assembly_contig_contig00007

whole_genome_shotgun_sequence Genome set $326237 \quad$ rep_cluster_475

I $\overline{C E} \quad$ Firmicutes

20298_2_28_Firmicutes_Clostridia_Clostridiales_NA_NA_.20298_2_28.7

Genome set $1690 \overline{2} 1$ N/A ICE Firmicutes

$142077^{7}$ 42_Firmicutes_Clostridia_Clostridiales_Lachnospiraceae_NA_.14207_

$7 \_42 . \overline{1} 6^{-}-$Genome Set $101356^{-}$N/A ICE Firmicutes 
UMGS871_ERR414245_bin.26 Bacteroidetes Bacteroidia Bacteroidales Bacteroi

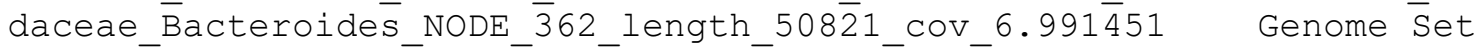

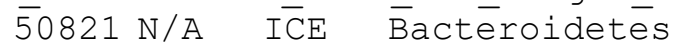

21673487 Firmicutes Clostridia Clostridiales Lachnospiraceae Dorea_.216 73 4_87.16 Genome Set $17400 \mathrm{~N} / \mathrm{A}$ - ICE Firmicutes

UMGGST̄767 SRR5275445 bin.35 Actinobacteria Actinobacteria Coriobacteriales

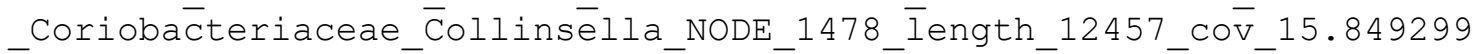

Genome set $1 \overline{2} 457 \mathrm{~N} / \mathrm{A}$ N/A Actinobacteria

20298_3_65_Firmicutes_Clostridia_Clostridiales_Lachnospiraceae_NA_.20298_

3_65. $\overline{2}-$ Genome Set 286851 N/A ICE Firmicutes

18048_1_69_Firmicutes_Clostridia_Clostridiales_Lachnospiraceae_NA_.18048_ $169 . \overline{3}{ }^{-}$- Genome Set 32366 N/A ${ }^{-}$ICE Firmicutes

UM̄GS1534_ERR1620311_bin.17_Bacteroidetes_Bacteroidia_Bacteroidales_Bacter oidaceae Bacteroide $\bar{s}$ NODE $\overline{1}$ length $64669 \overline{2}$ cov $6.5310 \overline{9} 4$ Genome Set
$64 \overline{6} 692$
$\mathrm{N} / \mathrm{A}^{-} \mathrm{IC} \overline{\mathrm{E}}-$ Bacteroidetes

UMGS262_SRR1761683_bin.19_Firmicutes_Clostridia_Clostridiales_Ruminococca ceae_NA_NODE_273_lēength_4 $8480 \_C O V+47.324750$ Genome set 48480 N/A ICE

Firmicutes

NZ_DS981457.1_Bacteroides_coprocola_DSM_17136_Scfld_02_30_genomic_scaffol d_whole_genome_shotgun_séquence Genome set $\overline{2}^{-} 8111 \overline{6}-\overline{\mathrm{N}} / \mathrm{A}$ ICE

Bacteroidetes

UMGS218_SRR5580022_bin.2_Bacteroidetes_Bacteroidia_Bacteroidales_Bacteroi

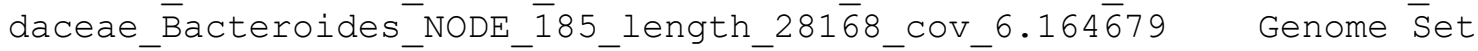

$\overline{2} 8168 \mathrm{~N} / \mathrm{A} \quad \mathrm{I} \overline{\mathrm{C}} \mathrm{B}$ Bacteroidetes

UMGS611_SRR5056929_bin.85_Tenericutes_NA_NA_NA_NA_NODE_2_length_372294_CO V $10.40 \overline{1} 685$ Genome Set $37 \overline{2} 294 \quad \mathrm{~N} / \mathrm{A}^{-} \overline{\mathrm{N}} / \mathrm{A}^{-}$Tenéricutés

UM̄GS1677 ERR1190831 bin.20 Firmicutes Clostridia Clostridiales Peptostrep

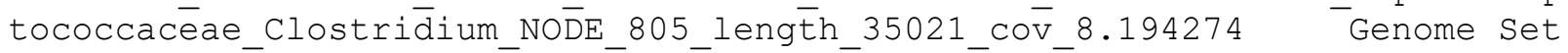
$35021 \overline{\mathrm{N}} / \mathrm{A} \quad \mathrm{N} / \mathrm{A}$ Firmicutes

8080_1_93_Firmicutes_Clostridia_Clostridiales_Lachnospiraceae_NA_ERS13819 1.8080_1_ 93.13 Genome Set $87 \overline{685}$ N/A ICE Firmicutes

UMGS511̄_DRR042316_bin.21_Actinobacteria_Actinobacteria_Coriobacteriales_C oriobact̄eriaceae_c̄ollinsēlla_NODE_21_leñgth_199171_cov_11.070869

Genome Set $199171 \quad \bar{N} / A-I C \overline{C E}$ Actinobactēria

14207_7_42_Firmicutes_Clostridia_Clostridiales_Lachnospiraceae_NA_.14207_ $742 . \overline{4} 8^{-}$- Genome Set $42764 \mathrm{~N} / \mathrm{A}^{-}$ICE Firmicutes

14207_7_37_Firmicutes_Clostridia_Clostridiales_Lachnospiraceae_Dorea_.142 $077^{7} \overline{3} 7.26^{-}$Genome Set 50901 N/A ${ }^{-}$ICE Firmicütes 20 $\overline{2} 9 \overline{8}$ 3_35_Firmicutes_Clostridia_Clostridiales_Lachnospiraceae_Blautia_.2 0298 3 $35 . \overline{3}$ Genome Set 239107 N/A ICE Firmicutes

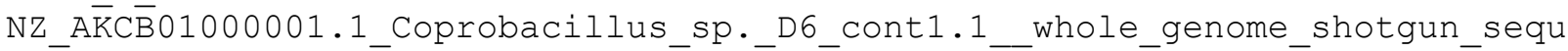
ence Genome set $\overline{2} 786946$ N/A ICE Tenericutes

13470_2_64_Bacteroidetes_Bacteroidia_Bacteroidales_Bacteroidaceae_Bactero

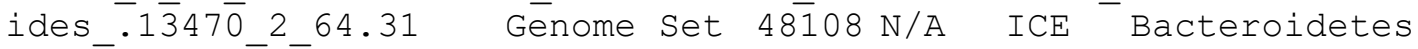

20298_3_81_Eirmicutes_Clostridia_Clostridiales_Lachnospiraceae_Blautia_.2 $0298 \overline{3} \overline{8} 1 . \overline{4}$ Genome Set 234766 - N/A ICE Firmicutes

20298_ 2 49_Firmicutes_Bacilli_Lactobacillales_Streptococcaceae_Streptococ cus_. $20 \overline{2} 98$ 2_49.1Genome set $\overline{6} 65222$ N/A ICE Firmicutes

UMGST193_DRRO 42356 bin.29 Tenericutes Mollicutes_Erysipelotrichales_Erysip elotrichaceae_Solōbacterium_NODE_271_length_68870_cov_7.194129 Genome Set $68870 \mathrm{~N} / \bar{A}$ ICE Tenericutēs

14207_7_90_Firmicutes_Clostridia_Clostridiales_NA_NA_.14207_7_90.7

Genome set $1514 \overline{9} 7 \quad$ N/A ${ }^{-}$ICE Firmicutes 
20298_2_28_Firmicutes_Clostridia_Clostridiales_NA_NA_.20298_2_28.2 Genome set $6498 \overline{1} 2 \quad$ N/A

NZ_LN913000.1_Ruminococcus_sp._AT10_genome_assembly_Ruminococcus_phoceens is scaffold_scaffold00004_whöle_genome_shotgun_sequenceGenome Set
710312
$\mathrm{N} / \mathrm{A}$
Firmicutes

UMGS796_ERR1190945_bin.62 Bacteroidetes Bacteroidia_Bacteroidales Prevote

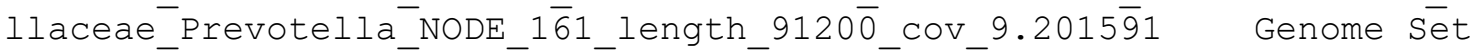

$9 \overline{1} 200 \mathrm{~N} / \mathrm{A} \quad \overline{\mathrm{C}} \mathrm{E} \quad \overline{\mathrm{B}} \mathrm{acte} \mathrm{e} \mathrm{r} i \mathrm{det} \overline{\mathrm{e}} \mathrm{s}$

UMGS282_ERR525699_bin.23_Bacteroidetes_Bacteroidia_Bacteroidales_Porphyro monadaceae_Parabacteroides_NODE_778_length_30050_cov_5.195699 Genome Set 30050 N/A ICE Bacteroidetes

13414_6_70_Firmicutes_Clostridia_Clostridiales_Lachnospiraceae_NA_.13414 $670 . \overline{1} 4^{-}$Genome Set $75536 \mathrm{~N} / \mathrm{A}$ ICE Firmicütes

127718_7_47_Firmicutes_Clostridia_Clostridiales_NA_NA_.12718_7_47.10 Genome Set $5676 \overline{7}$ N/A ICE ${ }^{-}$Firmicutes

UMGS1199_SRR3917587_bin.52_Tenericutes_NA_NA_NA_NA_NODE_719_length_25763_

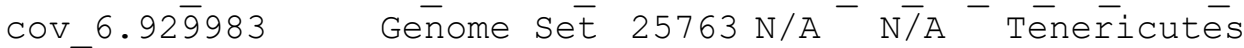

21673_4_18_Firmicutes_Clostridia_Clostridiales_NA_NA_.21673_4_18.18

Genome Set $5772 \overline{6}$ N/A ICE Firmicutes

13470_2_81_Firmicutes_Clostridia_Clostridiales_Lachnospiraceae_Blautia_.1 $3470 \overline{2} \overline{8} 1 . \overline{4} 2 \quad$ Genome Set $312 \overline{62}$ N/A N/A Firmicutes

NZ_JḦ $9 \overline{9} 2948.1$ Bacteroides_oleiciplenus_YIT_12058_genomic_scaffold_superco

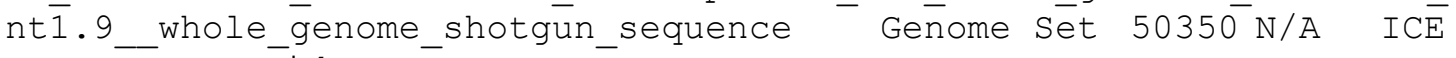
Bacteroidetes

20287_6_18_Firmicutes_Clostridia_Clostridiales_Lachnospiraceae_NA_.20287_ 6_18.10-Genome Set $86682 \mathrm{~N} / \mathrm{A}$ - ICE Firmicutes

UMMGS624_ERR011095_bin.1_Bacteroidetes_Bacteroidia_Bacteroidales_Bacteroid

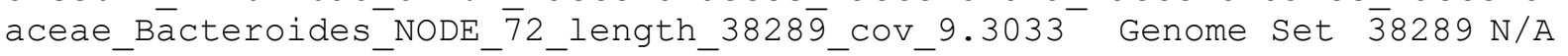
ICE Bacteroidetes

NZ_JH660546.1_Escherichia_sp._4_1_40B_supercont2.8_whole_genome_shotgun sequence Genome set $80 \overline{5} 75$ IncFiB, InCFIIA, IncFII Plasmid Proteobacteria

20287_6_9_Bacteroidetes_Bacteroidia_Bacteroidales_Bacteroidaceae_Bacteroi des_.20̄287_6_9.4 Genome Set 437212 N/A ICE Bacteroidetes

UMGS 200 SRR $6 \overline{0} 28624$ bin.16_Firmicutes_Clostridia_Clostridiales_Ruminococca

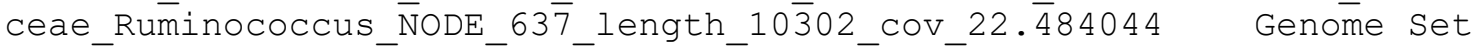

$$
10302 \mathrm{~N} / \mathrm{A} \quad \overline{\mathrm{I}} \mathrm{E} \text { Firmicutes }
$$

NZ_LN866274.1_Clostridium_sp._GD3_genome_assembly_Clostridium_phoceensis_ _sçaffold_scaf́fold00010_wholēenenome_shötgun_sequence Genome set
$327 \overline{9} 315$
N/A ICE Firmicutes

NZ_DS499701.1_Clostridium_scindens_ATCC_35704_Scfld_02_23_genomic_scaffol d_whole_genome_shotgun_sēquence Genome set $\overline{17302 \overline{3}}-\overline{\mathrm{N}} / \mathrm{A}$ ICE Firmicutes

UMGS803_SRR5127411_bin.10_Firmicutes_Clostridia_Clostridiales_Peptostrept

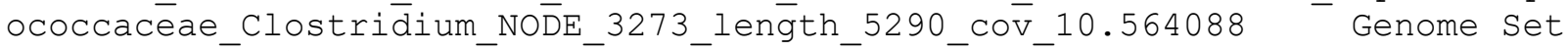
$5290^{-}$N/A N/A Firmicutes

UMGS200_SRR6028624_bin.16_Firmicutes_Clostridia_Clostridiales_Ruminococca

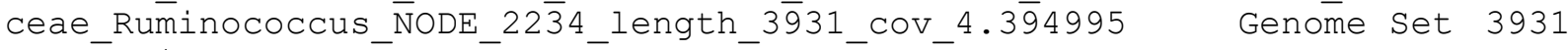

N/A ICE Firmicutes

UMGS771_SRR5580101_bin.4_Firmicutes_Clostridia_Clostridiales_Ruminococcac

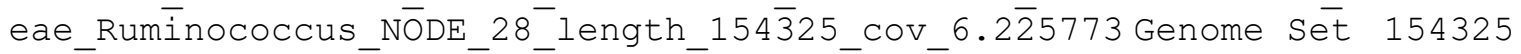

N/A ICE Firmicutes 
NZ_DS981478.1_Bacteroides_coprocola_DSM_17136_Scfld_02_51_genomic_scaffol d_whole_genome_shotgun_séquence Genome set $58798 \overline{\mathrm{N}} / \overline{\mathrm{A}} \overline{\mathrm{I}} \mathrm{CE}$

Bacteroidetes

UMGS1102 ERR688524 bin.55 Firmicutes Clostridia Clostridiales Lachnospira

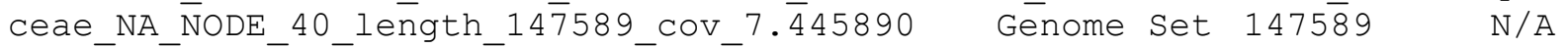

I $\overline{C E} \quad \overline{\text { Firmicutes }}$

NZ_KQ236744.1_Dorea_sp._D27_genomic_scaffold_supercont1.2_whole_genome_s hot̄gun sequence Geñome Set $1018203^{-}$N/A ICE Firmicutes

UMGS916_SRR1761681_bin.7_Firmicutes_Clostridia_Clostridiales_Ruminococcac eae_NA_NoDE_250_leñgth_36̄815_cov_17.458950 Genome set $368 \overline{1} 5$ N/A ICE

Firmicutes

NZ_DS981485.1_Bacteroides_coprocola_DSM_17136_Scfld_02_58_genomic_scaffol d_whole_genome_shotgun_sēquence Genome set $10186 \overline{4}$ ICE Bacteroidetes

13470 2 71 Tenericutes_Mollicutes_Erysipelotrichales_Erysipelotrichaceae NA_.1 $\overline{3} 4 \overline{7} 0 \overline{2}_{2} 71.5$ Genome Set $1894 \overline{13}$ N/A ICE Tenericutes

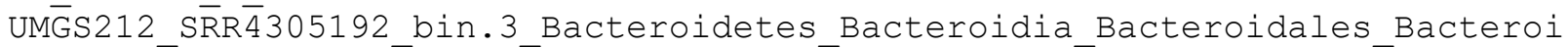

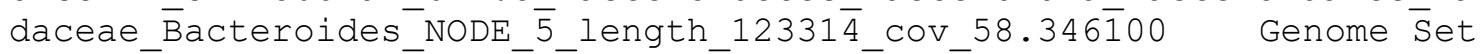

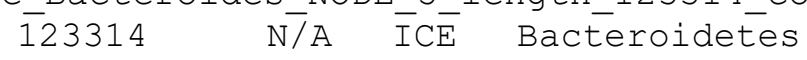

NZ_KB905488.1_Bacteroides_sp._HPS0048_genomic_scaffold_acKdP-

supercont1.2_whole_genomē_shōtgun_sequence Genome Sēt 1254863 N/A

ICE $\overline{\text { Bacteroidetes }}$

NZ_HE978577.1_Bacteroidales_bacterium_ph8_genomic_scaffold_scaffold00001

whole_genoméshotgun_sequence Genome set $2110561 \quad \mathrm{~N} / \mathrm{A} \quad$ ICE

Bacteroidetes

UMGS35_ERR525726_bin.20_Firmicutes_Clostridia_Clostridiales_Peptostreptoc

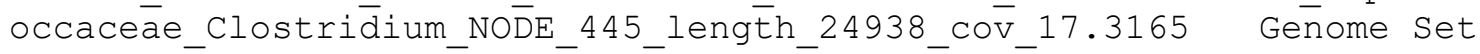

$24 \overline{9} 38 \mathrm{~N} / \mathrm{A} \quad \mathrm{ICE} \overline{-}^{-}$Firmicütes

NZ_KE150450.1_Actinomyces_sp._HPA0247_genomic_scaffold_acAqT-

supercont1.1_whole_genome_shōtgun_sequence Genome Sēt 1289359 N/A ICE Actinobacteria

NZ_EQ973223.1_Bacteroides_fragilis_3_1_12_supercont1.11_genomic_scaffold_ _whole_genome_shotgun_sequence Genome Set 37990 N/A ${ }^{-}$N/A Bacteroidetes

UMGS1021_ERR525737_bin.53_Tenericutes_NA_NA_NA_NA_NODE_1_length_582527_CO

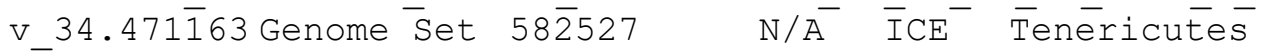

UMGGS230_SRR1761716_bin.48_Firmicutes_Clostridia_Clostridiales_Ruminococca ceae_NA_NODE_2_length_256835_COV_31.533663 Genome set $2568 \overline{3} 5$ N/A ICE Firmicutes

UMGS342_ERR688517_bin.19_Firmicutes_Clostridia_Clostridiales_NA_NA_NODE_2

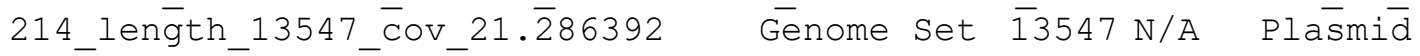

Firmicutes

NZ_HE578907.1_Anaerococcus_sp._JC48_genomic_scaffold_scaffold00001_whol e_genome_shotgun_sequence Genome Set $136 \overline{7} 741$ N/A ICE Firmicutes $1 \overline{3} 470$ 2_-93_Firmicutes_Clostridia_Clostridiales_Lachnospiraceae_NA_.13470_ $293 . \overline{2}{ }^{-}$- Genome Set $54726 \mathrm{~N} / \mathrm{A}^{-}$ICE Firmicütes

14207_7_50_Firmicutes_Clostridia_Clostridiales_NA_NA_.14207_7_50.20

Genome set $2898 \overline{0}$ N/A ICE Firmicutes

UMGS803_SRR5127411_bin.10_Firmicutes_Clostridia_Clostridiales_Peptostrept

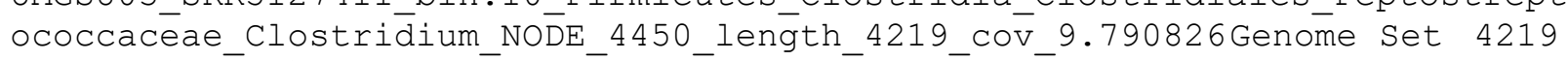
N/A N/A Firmicutes

NZ_GG730106.1_Bacteroides_sp._D20_genomic_scaffold_supercont1.2_whole_ge nome_shotgun_sequence Geñome Set $^{-} 1323254^{-}$N/A ${ }^{-}$ICE Bacteroidetes 
21673_4_87_Firmicutes_Clostridia_Clostridiales_Lachnospiraceae_Dorea_.216 73_4_87.9- Genome Set 120334 N/A ICE Firmicutes

NZ_DS483503.1_Faecalibacterium_prausnitzii_M21_2_Scfld_02_24_genomic_scaf

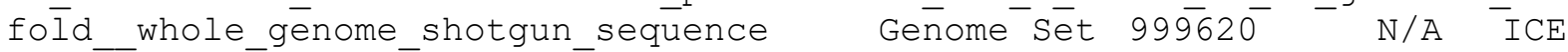
Firmicutes

NZ EQ973213.1 Bacteroides fragilis 3112 supercont1.1 genomic scaffold whole_genome_shotgun_sequence Genome Set 2558453 N/A ICE Bacteroidetes

18048_1_70_Firmicutes_Clostridia_Clostridiales_Ruminococcaceae_NA_.18048 $170 . \overline{1} 6^{-}$Genome Set $50829 \mathrm{~N} / \mathrm{A}^{-}$ICE Firmicütes 127718_7_56_Firmicutes_Bacilli_Lactobacillales_Lactobacillaceae_Lactobacil lus . $\overline{1} 2 \overline{7} 188^{-} 756.56$ - Genome Set 7846 N/A Plasmid Firmicutes UMG $\bar{S} 1135$ ERRR209469_bin.1_Firmicutes_Clostridia_Clostridiales_NA_NA_NODE_3 03 length $34553 \mathrm{cov} 6.02 \overline{7} 915$ Genome Set $34553 \overline{\mathrm{N}} / \mathrm{A}$ ICE Firmicute $\bar{s}$ NZ GL883880.1 Paraprevotella xylaniphila YIT 11841 genomic scaffold Scfld $30 \overline{3}$ _whole_geñome_shotgun_sequence Genome Set $^{-} 1269 \overline{70} \quad \mathrm{~N} / \mathrm{A} \quad$ ICE Bacteroidetes

UMGS1155 ERR209170 bin.2 Bacteroidetes Bacteroidia Bacteroidales Prevotel

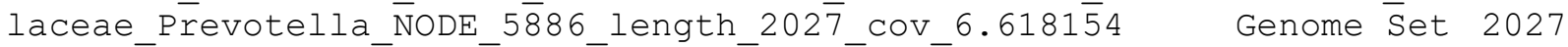
$\overline{\mathrm{N}} / \mathrm{A} \quad \mathrm{Plasmid} \quad$ Bactèroidetes

NZ_HG005174.1_Nesterenkonia_massiliensis_strain_NP1_whole_genome_shotgun sēquence Genome Set $4670 \overline{3}$ N/A Plasmid Actinobactería 20287_6_88_Firmicutes_Clostridia_Clostridiales_Lachnospiraceae_NA_.20287_ $688 . \overline{2}-$ Genome Set $476531-$ N/A ICE Firmicutes

UMMGS1268 ERR525890 bin.1 Firmicutes Clostridia Clostridiales Lachnospirac

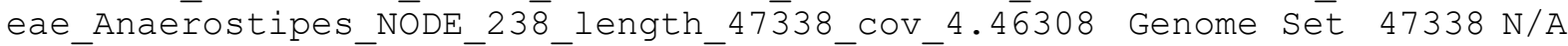

ICE Firmicutes

NZ_KQ033906.1_Parabacteroides_sp. HGS0025_genomic_scaffold_aczIssupercont1.5_whole_genome_shōtgun_sequence Genōme set $1 \overline{1} 0595$ N/A ICE Bacteroidetes

14207_7_87_Firmicutes_Clostridia_Clostridiales_NA_NA_.14207_7_87.18 Genome Set $8385 \overline{8}$ N/A ICE ${ }^{-}$Firmicutes

UMGS1777_SRR5275432_bin.13_Firmicutes_Clostridia_Clostridiales_Lachnospir aceae_NA_NODE_1472_- Iength_10910_cov_7.355136 Geñome set 10910 N/A ICE Firmicutes

20427425 Firmicutes Clostridia Clostridiales Lachnospiraceae Lachnobact erium . $204 \overline{2} 7425.16^{-}$Genome Set 73535 N/A ICE Firmicutes

UMGS $9 \overline{0} 4$ SRR1 $\overline{7} 6 \overline{1} 720$ bin. 5 Firmicutes Clostridia Clostridiales Lachnospirac

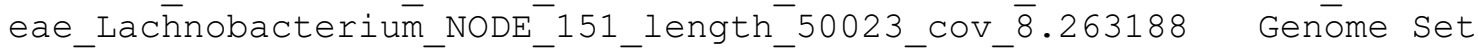
50023 N/A ICE Firmicutes

UMGS1165_ERR1913008_bin.26_Actinobacteria_Actinobacteria_Coriobacteriales _Coriobacteriaceae_Collinsēlla_NODE_214_lèngth_42534_cov_8.227924

Genome Set $4 \overline{2} 534$ N/A N/A Actinöbactería

NZ_GL892007.1_Dysgonomonas_mossii_DSM_22836_genomic_scaffold_supercont1.4 whole_genoméeshotgun_sequence Genome Set $40434 \overline{8} \quad$ N/A ${ }^{-}$ICE Bacteroidetes

UMGS 46 ERR209453 bin.40_Firmicutes Clostridia Clostridiales Ruminococcac

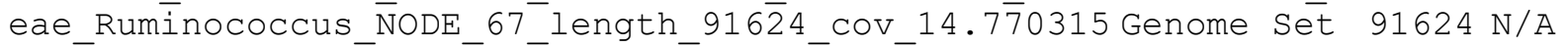
ICE Firmicutes

14207_7_9_Firmicutes_Clostridia_Clostridiales_Lachnospiraceae_Lachnospira $.142 \overline{0} 7^{-}{ }^{-}-9.11$ Genome Set $92 \overline{1} 47$ N/A ICE Firmicutes 8080_1_69_Firmicutes_Clostridia_Clostridiales_Lachnospiraceae_NA_ERS13816 7.8080_1_-69.9 Genome Set $10 \overline{1153}$ N/A ICE Firmicutes 
UMGS871_ERR414245_bin.26 Bacteroidetes Bacteroidia Bacteroidales Bacteroi

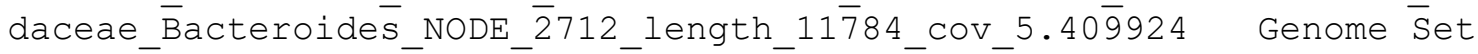

$\overline{1} 1784 \mathrm{~N} / \mathrm{A} \quad \overline{\mathrm{C}} \mathrm{E}$ Bacteroidetes

UMGS607 ERR526073 bin.39 Bacteroidetes Flavobacteriia Flavobacteriales Fl avobactēriaceae_F̄̄avobacterium_NODE_688_length_24371_cov_3.876789

Genome set 24371 N/A ICE Bacteroidetes

NZ_LT160637.1_Neglecta_timonensis_strain_SN17_whole_genome_shotgun_seque ncēe Genome Set $22725 \overline{0}$ N/A ICE Firmicutes

UMGS803_SRR5127411_bin.10_Firmicutes_Clostridia_Clostridiales_Peptostrept

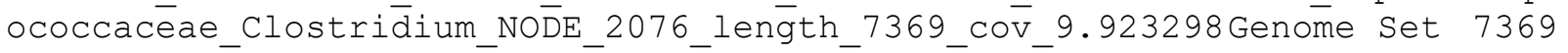

N/A ICE Firmicutes

14207_7_83_Firmicutes_Clostridia_Clostridiales_Lachnospiraceae_Roseburia_ $.1420 \overline{7} \overline{7} 8 \overline{3} .24$ Genome set $700 \overline{20}$ N/A ICE Firmicutes

UMGS1710 ERR1293845 bin.21 Bacteroidetes Bacteroidia Bacteroidales Bacter

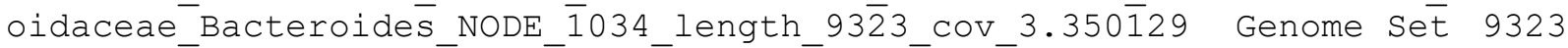
$\mathrm{N} / \overline{\mathrm{A}} \quad \mathrm{N} / \mathrm{A} \quad$ Bacteroidetes

UMGS791_ERR1190658_bin.15_Firmicutes_Negativicutes_Selenomonadales_Acidam

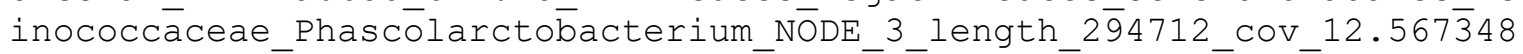

Genome Set 294712 N/A ICE Firmicutes

UMGS174_SRR1761690_bin.37_Firmicutes_Clostridia_Clostridiales_Ruminococca ceae_NA_NODE_72_length_96072_cov_93. 906340 Genome set $9607 \overline{2}$ N/A ICE

Firmicutes

21673_4_46_Bacteroidetes_Bacteroidia_Bacteroidales_Bacteroidaceae_Bactero ides_.21 $67 \overline{3} 446.19$ Genome set $71 \overline{3} 44 \mathrm{~N} / \mathrm{A}$ ICE Bacteroidetes

$2167 \overline{3}$ 4_33_Bacteroidetes_Bacteroidia_Bacteroidales_Porphyromonadaceae_Par abactēeroidés_.21673_4_33.6 Genome set 285921 N/A ICE

Bacteroidetes

21673_4_53_Firmicutes_Clostridia_Clostridiales_Lachnospiraceae_Blautia_.2 $1673 \overline{4} \overline{5} 3 . \overline{3}$ Genome set 391546 N/A ICE Firmicutes

NZ_EQ $9 \overline{7} 3216.1$ Bacteroides_fragilis_3_1_12_supercont1.4_genomic_scaffold

whōle_genome_shotgun_sequence Genome Set 501393 rep_ciluster_663

ICE Bacteroidetes

UMGS564_SRR2726136_bin.31_Firmicutes_Clostridia_Clostridiales_Lachnospira

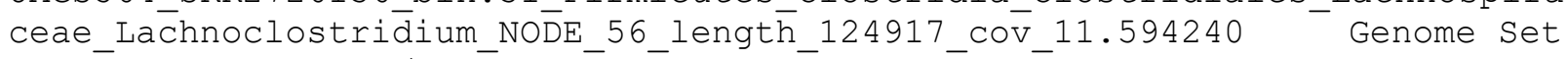
124917
N/A ICE Firmicutes

13470_2_65_Bacteroidetes_Bacteroidia_Bacteroidales_Bacteroidaceae_Bactero ides_.13470_2_65.17 Genome Set 139608 N/A ICE Bacteroidetes

NZ_DS996920.1_[Bacteroides]_pectinophilus_ATCC_43243_Scfld_02_0_genomic_s caffold_whole_enome_shotgun_sequence Genome Set 29332 N/A $-\overline{\text { ICE }}$

Firmicutes

UMGS725_SRR6054488_bin.26_Firmicutes_Clostridia_Clostridiales_Peptostrept

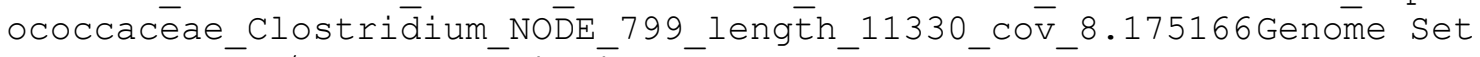

$11330^{-} \mathrm{N} / \mathrm{A}$ ICE Firmícutēs

UMGS698_ERR414252_bin.16_Bacteroidetes_Bacteroidia_Bacteroidales_Bacteroi daceae_Bacteroides_NODE_$\overline{7} 3$ _length_119172_Cov_33.443060 Genome $\bar{S} e t$

$\overline{1} 19172 \quad \mathrm{~N} / \mathrm{A} \quad \overline{\mathrm{I}} \mathrm{CE}^{-}$Bacteroidetes

NZ_ACCL02000002.1_Marvinbryantia_formatexigens_DSM_14469_B_formatexigens1.0.1_Cont1.1_whole_genome_shotgun_sequence Genome Set $3 \overline{1} 9694$ N/A ICE Firmicutes

20427_4_25_Firmicutes_Clostridia_Clostridiales_Lachnospiraceae_Lachnobact erium_. $204 \overline{2} 7$ 4_25.18 Genome Set 52463 N/A ICE Firmicutes UMGS $4 \overline{6} 9$ _SRR5 $\overline{2} 7 \overline{5} 479$ _bin.2_Bacteroidetes_Bacteroidia_Bacteroidales_NA_NA_NO DE_32_léngth_130808_cov_27.579635 Genome Set $1308 \overline{08}$ N/A I $\overline{C E}$

Bacteroidetes 
UMGS372_SRR6028394_bin.25_Bacteroidetes_Bacteroidia_Bacteroidales_Rikenel laceae_Ālistipes_NŌDE_3_lēength_261985_cov_12.244752 Genome set $26 \overline{1} 985$ $\overline{\mathrm{N}} / \mathrm{A}$ ICE Bacteroidetes

21673 4 18 Firmicutes Clostridia_Clostridiales_NA NA_.21673 4 18.7 Genome Set $2157 \overline{4} 1 \quad$ N/A ${ }^{-}$ICE Firmicütes

UMGS1096_SRR5057037_bin.59_Firmicutes_Clostridia_Clostridiales_Eubacteria

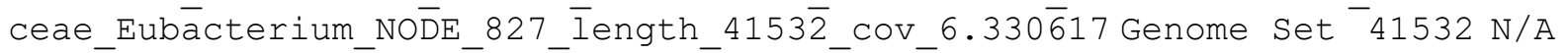

N/A Firmicutes

UMGS1546_SRR5056698_bin.29_Actinobacteria_Actinobacteria_Coriobacteriales _Coriobacteriaceae_Collinsēlla_NODE_1058_Iength_27934_cov _15.550701

Genome set $2 \overline{7} 934$ N/A ICE Actinobacteria

UMGS2011_ERR2013565_bin.54_Firmicutes_Clostridia_Clostridiales_Peptostrep

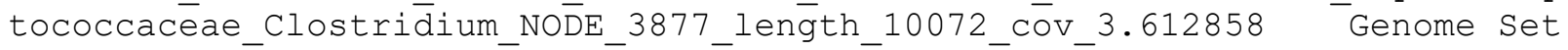
$10072 \overline{\mathrm{N}} / \mathrm{A} \quad \mathrm{N} / \mathrm{A} \quad \overline{\mathrm{F}} \mathrm{irmi} \bar{c} u t e s$

12718_7_94_Firmicutes_Clostridia_Clostridiales_Lachnospiraceae_NA_.12718_ 7 94.1̄ ${ }^{-}$- Genome Set 70133 N/A ${ }^{-}$ICE Firmicütes

200298_3_39_Firmicutes_Clostridia_Clostridiales_NA_NA_.20298_3_39.2

Genome set $4791 \overline{7} 8 \quad$ N/A ${ }^{-}$ICE Firmicutes

UMGS1367_SRR4408158_bin.11_Firmicutes_Bacilli_Lactobacillales_Enterococca

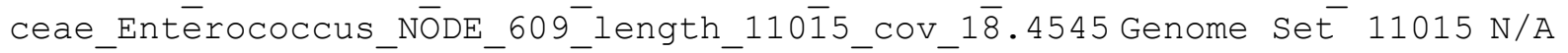

Plasmid $\overline{\text { Firmicutes }}$

NZ_GG662012.1_Coprococcus_comes_ATCC_27758_genomic_scaffold_Scfld7_whole gēnome_shotgünsequence - Genome Set $30 \overline{7} 897$ N/A ICE Firmicutes 13414_6_34_Firmicutes_Clostridia_Clostridiales_Peptostreptococcaceae_Clos

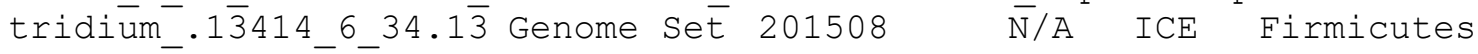

UMGS363_ERR130 $\overline{5} 900$ bin.6_Firmicutes_Clostridia_Clostridiales_Ruminococcac

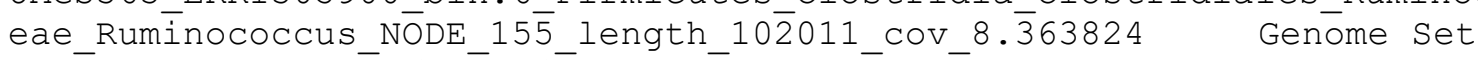
102011
N/A N/A Firmicutes

8080_1_78_Firmicutes_Clostridia_Clostridiales_Peptostreptococcaceae_Clost ridiü ERS̄ $138176.808 \overline{0} 178.32$ Genome Set 83786 N/A ICE Firmicutes 13414_- 6_17_Firmicutes_Clostridia_Clostridiales_Lachnospiraceae_RuminococC

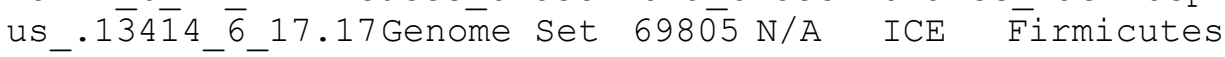

UMGGS1126_E ERR 414269 bin.20_Bacteroidetes_Bacteroidia_Bacteroidales_Porphyr omonadacēae_NA_NODE_7451_īength_3988_cov_5.113654 Genome set 3988 N/A

Plasmid - Bacteroìdetes

20298_3_30_Bacteroidetes_Bacteroidia_Bacteroidales_Porphyromonadaceae_NA_ $.2029 \overline{8} \overline{3} 3 \overline{0} .12$ Genome Set $74664 \mathrm{~N} / \overline{\mathrm{A}}$ ICE Bacteroidetes

UMGS1684_ERR011192_bin.15_Bacteroidetes_Bacteroidia_Bacteroidales_Prevote

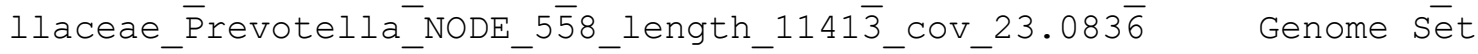

$1 \overline{1} 413 \mathrm{~N} / \mathrm{A} \quad \overline{\mathrm{C}} \mathrm{E}$ Bacteroidetés

UMGS1111_ERR414291_bin.37_Bacteroidetes_Bacteroidia_Bacteroidales_Porphyr omonadacēae_NA_NODE_624_lēength_35281_cov_3.775649 Genome set $35 \overline{2} 81$ N/A

ICE Bacteroidetes

20287_6_88_Firmicutes_Clostridia_Clostridiales_Lachnospiraceae_NA_.20287_ $688 . \overline{8}-$ Genome Set 280286 N/A ICE Firmicutes

NZ̄_LN909053.1_Paenibacillus_sp._MT18_genome_assembly_Paenibacillus_rubiin fantis_scaffold_scaffold00007_whole_genomé_shotgun_sequence Genome set $\overline{2414}$ N/A N/A Firmicutes

NZ_DS547029.1_Clostridium_sp._SS2_1_Scfld_03_33_genomic_scaffold_whole_g enomesshotgun_sequence Geñome ${ }^{-}$Set $587063-{ }_{\text {N/A ICE }}^{-}$Firmicutes NZ_JH376876.1_Clostridium_clostridioforme_2_1_49FAA_genomic_scaffold_supe rcōnt1.7_whole_genome_shōtgun_sequence Gēōomē set 268514 - N/A ICE Firmicutes 
UMGS1064_ERR321174_bin.8_Bacteroidetes_Bacteroidia_Bacteroidales_Porphyro monadaceāe_Parabact̄eroidès_NODE_409_length_14853_cöv_3.68692 Génome set $1485 \overline{3} \mathrm{~N} / \mathrm{A} \quad \mathrm{ICE}$ Bacteroīetes

UMGS1938 SRR5963258 bin.3 Bacteroidetes Bacteroidia Bacteroidales Porphyr

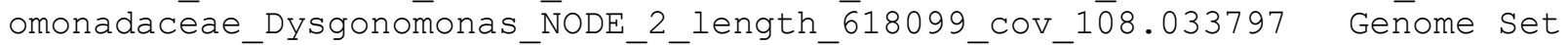
$61809 \overline{9}$
N/A I $\bar{C} E$ Bacteroidetes

UMGS199 SRR4408188_bin.8_Firmicutes_Clostridia_Clostridiales_Lachnospirac

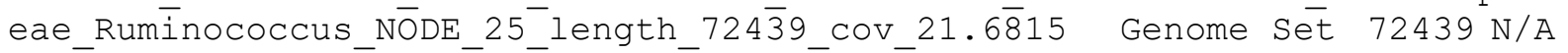

ICE Firmicutes

20287_6_88_Firmicutes_Clostridia_Clostridiales_Lachnospiraceae_NA_.20287_

$688 . \overline{1} 7^{-}$Genome Set $120030-$ N/A ICE Firmicutes

$1 \overline{3} 414$ 6_33_Firmicutes_Clostridia_Clostridiales_Lachnospiraceae_NA_.13414_ 6_33. ${ }^{-}{ }^{-}$- Genome Set 291015 - N/A ICE Firmicutes

UMMGS1234_SRR3582140_bin.13_Firmicutes_Clostridia_Clostridiales_NA_NA_NODE

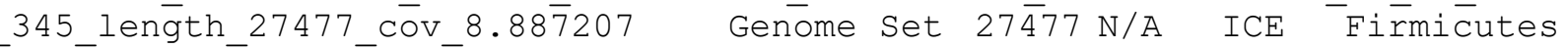
$\overline{1} 341 \overline{4}$ 6_60_Firmicütes_Clostridia_Clostridiales_NA_NA_.13414_6_60.8

$$
\text { Genome set } 1365 \overline{1} 5 \quad \text { N/A ICE Firmicutes }
$$

UMGS1297_SRR3917587_bin.20_Firmicutes_Clostridia_Clostridiales_Lachnospir aceae_NA_NODE_10_leñgth_259̄892_Cov_26.361808 Geñome Set $25989 \overline{2}$ N/A ICE Firmicutes

NZ_LT594621.1_Culturomica_massiliensis_strain_Marseille-

P2698_whole_genome_shotgü_sequence Genomē Set 4124789 N/A ICE

12718_7_84_Firmicutes_Clostridia_Clostridiales_Ruminococcaceae_NA_.12718_ $784 . \overline{5}-$ Genome Set 289744 N/A ICE Firmicutes

UMMGS161_ERR525920_bin.17_Firmicutes_Clostridia_Clostridiales_Lachnospirac

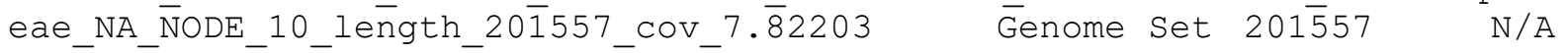
ICE Firmicutes

20287_6_6_Proteobacteria_Gammaproteobacteria_Enterobacteriales_Enterobact

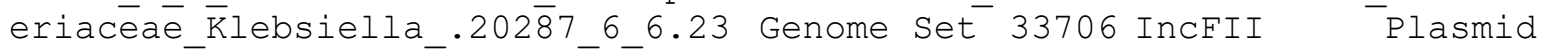
Proteobacteria

11861_6_58_Firmicutes_Clostridia_Clostridiales_Lachnospiraceae_Blautia_.1 $1861 \overline{6} \overline{5} 8 . \overline{2} 3$ Genome set $496 \overline{68}$ N/A ICE Firmicutes

$1347 \overline{0} \overline{2}$ 81_Firmicutes_Clostridia_Clostridiales_Lachnospiraceae_Blautia_.1 $3470 \overline{2}-\overline{8} 1 . \overline{5}$ Genome Set 146457 N/A ICE Firmicutes

UMGST̄234_SRR3582140_bin.13_Firmicutes_Clostridia_Clostridiales_NA_NA_NODE 112_length_54952_cov_11.258338 Genome set $54 \overline{95} 2 \mathrm{~N} / \mathrm{A}$ ICE Firmicutes

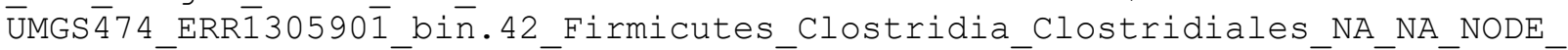

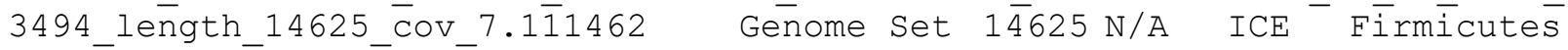
UMGS $\overline{6} 98$ ERR 414252 bin.16_Bacteroidetes_Bacteroidia_Bacteroidales_Bacteroi daceae_Bacteroides_NODE_66_length_122552_cov_34.078230 Genome Set

$122552 \quad \mathrm{~N} / \mathrm{A} \quad \overline{\mathrm{ICE}}{ }^{-}$Bacteroidetes

NZ_GL882693.1_Bacteroides_fluxus_YIT_12057_genomic_scaffold_Scfld385_who le_genome_shoțgun_sequence Genome Set $96 \overline{5} 35 \mathrm{~N} / \mathrm{A}{ }^{-}$ICE Bacteroidetes UMGS50_ERRE1293691_bin.12_Bacteroidetes_Bacteroidia_Bacteroidales_Rikenell aceae_Ālistipes_NŌDE_1_lēength_459252_cov_26.627145 ${ }^{-}$Genome Set $4 \overline{5} 9252$

N/A ICE Bactēeroidetes

UMGS35_ERR525726_bin.20_Firmicutes_Clostridia_Clostridiales_Peptostreptoc

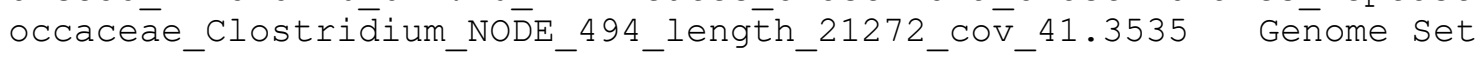
$21 \overline{2} 72 \mathrm{~N} / \mathrm{A}$ ICE Firmicutes

UMGS785_ERR1297697_bin.13_Firmicutes_Clostridia_Clostridiales_NA_NA_NODE 133_lenḡth_81764_Cov_7.997981 Genome set $8 \overline{1764}$ N/A ICE Firmínutes 
NZ_KQ235848.1_[Clostridium]_bolteae_WAL-

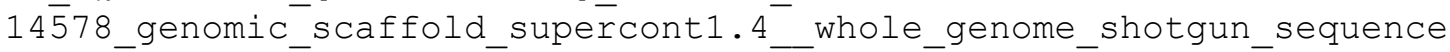

Genome Set $62535 \overline{5} \quad$ N/A ICE Firmicutes

NZ_LN877939.1_Gabonia_massiliensis_strain_GM3_whole_genome_shotgun_seque ncē Genome set $1352 \overline{0} 85$ N/A ICE Bäcteroidetes

8080_1_78_Firmicutes_Clostridia_Clostridiales_Peptostreptococcaceae_Clost ridium_ERS̄138176.8080_1_78.37- Genome set 72352 N/A ICE Firmicutes UMGS $46 \overline{7}$ SRR5127501 bin. $\overline{3} 1$ Actinobacteria Actinobacteria Coriobacteriales Coriobacteriaceae_Collinsēella_NODE_139_lēngth_52930_cov_10.242686

Genome set $52930 \mathrm{~N} / \mathrm{A}$ ICE Äctinobacteria

NZ_GL882697.1_Bacteroides_fluxus_YIT_12057_genomic_scaffold_Scfld446_who le_genome_shot̄gun_sequence $\bar{e}$ Genome Set $13 \overline{2} 721-$ N/A ICE

Bacteroidetes

UMGS991 ERR1620299 bin.39 Firmicutes Clostridia Clostridiales Ruminococca

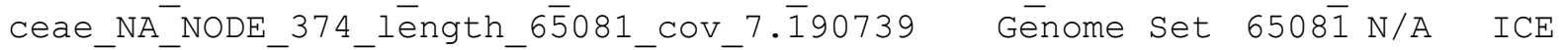

Firmicutes

NZ JH815222.1 Clostridium sp. 72 43FAA genomic scaffold supercont2.3 wh olēe_genome_shōtgun_sequence Genome set $3226588^{-}$N/A ICE Firmicutes UMG $\bar{S} 531$ ERR 1018228 bin.33_Tenericutes_Mollicutes_NA_NA_NA_NODE_159_length

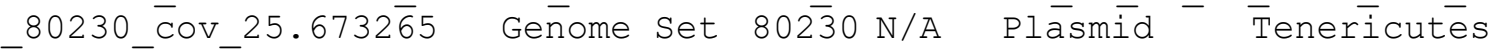
$\bar{N} Z$ KI2 $\overline{6} 044 \overline{9} .1$ Ruminococcus_callidus_ATCC_27760_genomic_scaffold_Scaffold5 $49^{-}$whole_genome_shotgun_séquence Genome Set $\overline{118054}$ - N/A İCE

Firmicutes

NZ_JH379466.1_Prevotella_stercorea_DSM_18206_genomic_scaffold_Scfld1385

whöle_genome_shotgun_sequence Genome Set 17608 N/A ICE

Bacteroidetes

UMGS120_ERR1018284 bin.6_Bacteroidetes_Bacteroidia_Bacteroidales Rikenell

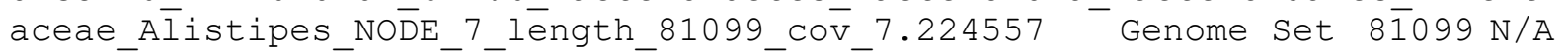
ICE Bactéeroidetes

NZ_DS499718.1_Clostridium_scindens_ATCC_35704_Scfld_02_40_genomiC_scaffol d_whole_genome_shotgun_sequence Genome Set $38327 \overline{7}-\overline{\mathrm{N}} / \mathrm{A}$ ICE Firmicutes

20287_6_37_Firmicutes_Clostridia_Clostridiales_NA_NA_.20287_6_37.61 Genome set 5656 N/A Plasmid Firmicutes

NZ_HE611014.1_Senegalimassilia_anaerobia_JC110_type_strain_JC110T_genom ic_scaffold_s scaffold00001_whōle_genome_shotgun_sequenceGē̄ome set
782271
$\mathrm{N} / \mathrm{A} \quad \mathrm{ICE}$
Actinobacteria

UMGS1717_SRR5057039_bin.40_Firmicutes_Clostridia_Clostridiales_Oscillospi

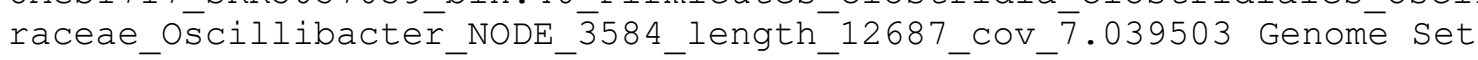

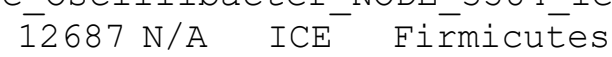

14207_7_86_Firmicutes_Clostridia_Clostridiales_NA_NA_.14207_7_86.2

Genome set $6190 \overline{5} 4$ N/A ICE Firmicutes

21673_4_87_Firmicutes_Clostridia_Clostridiales_Lachnospiraceae_Dorea_.216 73_4_87.1- Genome Set 949954 - N/A ICE Firmicutes

13 $\overline{4} 1 \overline{4}$ 6_20_Firmicutes_Clostridia_Clostridiales_Lachnospiraceae_Blautia_.1 $3414 \overline{6} \overline{2} 0 . \overline{1}$ Genome Set $625992 \quad$ N/A ICE Firmicutes

$2167 \overline{3}$ 4_37_Firmicutes_Clostridia_Clostridiales_Lachnospiraceae_NA_.21673_ $437 . \overline{7}-$ Genome Set $157631-$ N/A ICE Firmicutes

14 207 7_56_Firmicutes_Clostridia_Clostridiales_NA_NA_.14207_7_56.37

Genome set $1195 \overline{4}$ N/A ICE Firmicutes

NZ_KQ033909.1_Parabacteroides_sp._HGS0025_genomic_scaffold_aczIs-

supercont1.8_whole_genome_shotgun_sequence Genome set 30788 N/A ICE Bacteroidetes 
UMGS342_ERR688517_bin.19_Firmicutes_Clostridia_Clostridiales_NA_NA_NODE_2

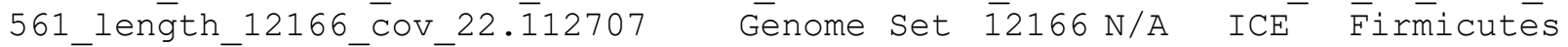
UMG $\bar{S} 1315$ SRRR21553̄18_bin.4_Firmicutes_Clostridia_Clostridiales_NA_NA_NODE

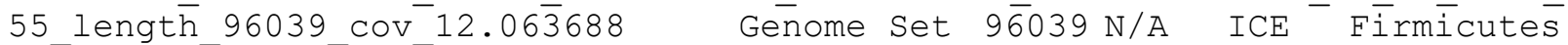
UMGSS601_SRR6028332_bin.7_Bacteroidetes_Bacteroidia_Bacteroidales_Porphyro monadacéae_NA_NODE_4_length_145256_cov_17.874023 Genome set $1 \overline{4} 5256$

$\mathrm{N} / \mathrm{A}$ ICE Bäteroidēes

13414_6_20_Firmicutes_Clostridia_Clostridiales_Lachnospiraceae_Blautia_.1 $3414 \overline{6} \overline{2} 0 . \overline{6}$ Genome Set $212672-$ N/A ICE Firmicutes $1420 \overline{7} \overline{6}$ 9_Bacteroidetes_Bacteroidia_Bacteroidales_Bacteroidaceae_Bacteroi des. $\overline{1} 4 \overline{2} 0 \overline{7} 6$ 9.52 Genome Set $23983 \mathrm{~N} / \mathrm{A}$ ICE Bacteroidetes

UMGS̄ $1540 \_S \bar{R} R \overline{3} 993002$ bin.12_Firmicutes_Clostridia_Clostridiales_NA_NA_NODE 7617 leñgth 6459 cóv $3.76 \overline{5} 121$ Genome set $64 \overline{59}$ N/A N/A Firmicutes

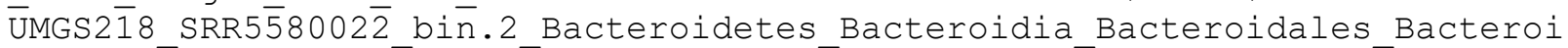

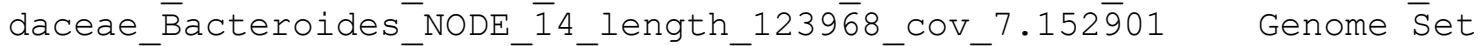
123968
$\mathrm{N} / \mathrm{A} \quad \overline{\mathrm{I}} \mathrm{CE}-$ Bacteroidetes

UMGS1151_ERR414319_bin.36_Firmicutes_Clostridia_Clostridiales_NA_NA_NODE

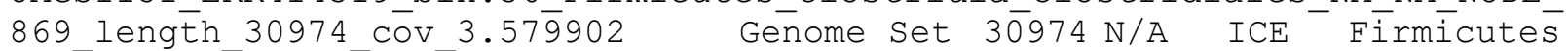
UMGST328_ERR $12936 \overline{1} 0 \_b \bar{i}$.26_Bacteroidetes_Bacteroidia_Bacteroidales_Bactero

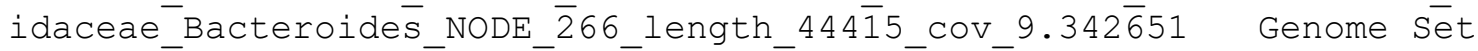

$4 \overline{4} 415 \mathrm{~N} / \mathrm{A}$ ICE Bacteroidetes

UMGS375_DRR042341_bin.5_Actinobacteria_Actinobacteria_Coriobacteriales_Co riobactēeriaceae_Cōllinsēlla_NODE_44_leñgth_94556_cov_ī12.667873 Genome Set $94556 \mathrm{~N} / \mathrm{A}$ ICE Actin̄obactería

12718_7_73_Firmicutes_Clostridia_Clostridiales_Lachnospiraceae_NA_.12718_ $773 . \overline{6}-$ Genome Set $356163-$ N/A ICE Firmicutes

UM̄GS1558_ERR1578626_bin.68_Actinobacteria_Actinobacteria_Coriobacteriales _Coriobacteriaceae_c̄ollinsēlla_NODE_457_lèngth_51337_cov_5.134219

Genome set $5 \overline{1} 337$ N/A ICE ACtinobactería

UMGS335_ERR1190610_bin.12_Firmicutes_Clostridia_Clostridiales_Lachnospira ceae_Blāutia_NODE_ī108_length_7565_cov_4.89587- Genome set 7565 N/A

ICE Firmicutes

20298_3_62_Firmicutes_Clostridia_Clostridiales_Peptostreptococcaceae_Clos

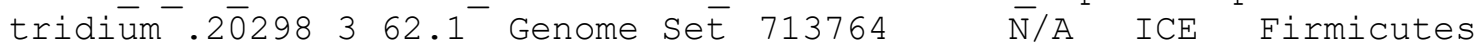

UMGS57_SRR2155 $\overline{3} 6 \overline{1}$ bin.23_Firmicutes_Clostridia_Clostridiales_NA_NA_NODE_6 43 length_19055_cov_8.986579 Genome Set 19055 N/A ICE Firmicutes UMḠS1199_S SRR3917587_bin.52_Tenericutes_NA_NA_NA_NA_NODE_502_length_34326_

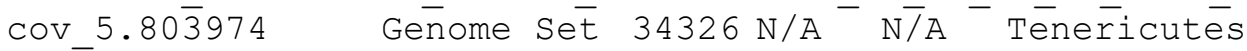

134 $\overline{7} 0$ 2_93_Firmicutes_Clostridia_Clostridiales_Lachnospiraceae_NA_.13470 2 293. $\overline{3}^{-}$- Genome Set $21187 \mathrm{~N} / \mathrm{A}$ - Plasmid Firmicutes

UM̄GS1607_SRR6054490_bin.8_Firmicutes_Clostridia_Clostridiales_Lachnospira ceae_NA_NoDE_192_leñgth_40723_Cov_11.253000 Genome set $4072 \overline{3}$ N/A ICE

Fìrmicütes

NZ_KI271189.1_Eubacterium_ramulus_ATCC_29099_genomic_scaffold_Scaffold864 whole genomé shotgun sequence Genome Set 1691 N/A N/A Firmicutes UMGS165ī_SRR39177587_biñ.7_Firmicutes_Clostridia_Clostridiales_NA_NA_NODE

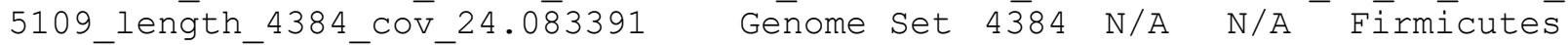
UMGST̄628_SRR̄5056930_bin.3_Firmicutes_Clostridia_Clostridiales_Lachnospira ceae_NA_NODE_1639_lēength_177344_COV_4.012956 Genome set $1734 \overline{4}$ N/A ICE Firmicutes

UMGS1488_SRR5057076_bin.64_Firmicutes_Clostridia_Clostridiales_Eubacteria

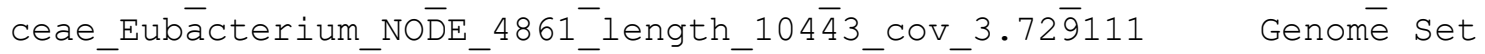
$10443 \mathrm{~N} / \mathrm{A}$ ICE Firmicutes 
UMGS669_SRR5275456_bin.26_Firmicutes_Clostridia_Clostridiales_Lachnospira

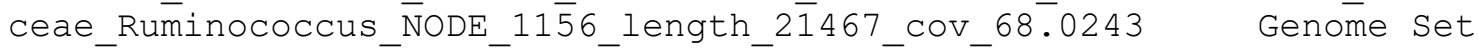

$21467 \mathrm{~N} / \mathrm{A} \overline{\mathrm{I}} \mathrm{CE}$ Firmicutes

UMGS658 SRR2155387 bin.7 Firmicutes Clostridia Clostridiales NA NA NODE 1 06 length $80246 \mathrm{cov} 21.1 \overline{4} 42$ Genome Set $80246 \overline{\mathrm{N}} / \mathrm{A}$ ICE Firmicutes

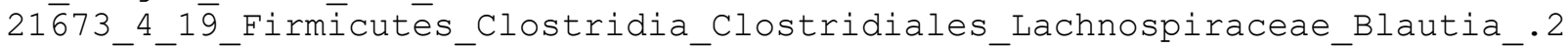
$1673 \overline{4} \overline{1} 9 . \overline{2} 4$ Genome set $358 \overline{60} \mathrm{~N} / \mathrm{A}$ ICE Firmicutes

UMGS $\overline{9} 2 \overline{9}$ SRR6054493_bin.5_Firmicutes_Clostridia_Clostridiales_NA_NA_NODE_2 84 length_18339_cov_38.253336 Genome set $\overline{1} 8339$ N/A ICE ${ }^{-} \overline{\text { Firmicutes }}$ UMGGS1534_ERR1620311_bin.17_Bacteroidetes_Bacteroidia_Bacteroidales_Bacter

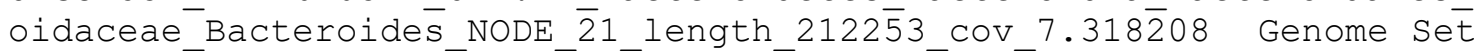

\section{$21 \overline{2} 253 \quad \mathrm{~N} / \mathrm{A}^{-} \quad \mathrm{IC} \overline{\mathrm{E}}$ Bacteroidetes}

UMGS476 SRR6028211 bin.4 Bacteroidetes Bacteroidia Bacteroidales Prevotel laceae $\bar{P}$ revotella $\bar{N} O D E$ 1012 length $802 \overline{7}$ cov $53.958 \overline{7} 31$ Genome Set 8027

$\overline{\mathrm{N}} / \mathrm{A} \quad \mathrm{ICE} \quad \overline{\mathrm{B}} \mathrm{acte} \overline{\mathrm{r}} \mathrm{ide} \bar{t} \mathrm{es}$

NZ_LN898211.1_Olsenella_sp._SIT9_genome_assembly_Olsenella_massiliensis scāffold_scaff́lold00013_whole_e_geñome_shötgun_sequence Genome set
$90 \overline{3} 327$
$\mathrm{N} / \mathrm{A}$ ICE Actinobacteria

18048_2_82_Firmicutes_Clostridia_Clostridiales_NA_NA_.18048_2_82.28

Genome Set $4452 \overline{9}$ N/A ICE ${ }^{-}$Firmicutes

NZ_GG730309.1_Clostridium_sp._M62_1_genomic_scaffold_Scfld0_whole_genome sh̄otgun sequence Genome Set $\overline{9477 \overline{3}}$ - N/A ICE Firmicutes

8080_1_72_Eirmicutes_Clostridia_Clostridiales_Lachnospiraceae_Blautia_ERS $1381 \overline{70} .80 \overline{8} 0$ 1_72.1 - Genome Sét 896810 N/A ICE Firmicutes

20298_3_59_Firmicutes_Clostridia_Clostridiales_NA_NA_.20298_3_59.6

$$
\text { Genome set } 5422 \overline{1} 9 \text { N/A ICE Firmicutes }
$$

13414_6_39_Firmicutes_Clostridia_Clostridiales_Ruminococcaceae_NA_.13414_ 6_39. $\overline{6}^{-}$- Genome Set 172853 N/A ICE Firmicutes

UMMGS1684_ERR011192_bin.15 Bacteroidetes Bacteroidia_Bacteroidales Prevote llaceae_Prevotella_NODE_37 _length_67171_COV_22.1585 Genome Set $67 \overline{1} 71$ N/A

ICE Bacteroidetes

UMGS1045_SRR3108056_bin.25_Tenericutes_NA_NA_NA_NA_NODE_774_length_36912_

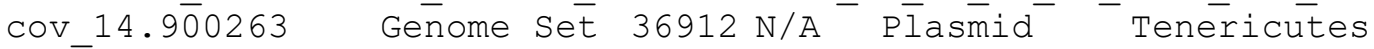

NZ_CYUJ01000002.1_Neofamilia_massiliensis_strain_SIT13_whole_genome_shot gun_sequence Genome set 940216 rep_cluster_16 $\overline{63}$ ICE Firmicutes 134144_6_37_Firmicutes_Clostridia_Clostridiales_NA_NA $\bar{A}+13414$ 6_37.3

$$
\text { Genome set } 1745 \overline{6} 7 \quad \text { N/A }- \text { ICE Firmicute } \bar{s}
$$

13414_6_67_Firmicutes_Clostridia_Clostridiales_Lachnospiraceae_NA_.13414_ 6_67. ${ }^{-}-$Genome Set 148362 N/A ICE Firmicutes

UMGS1369_SRR5050586_bin.6_Firmicutes_Clostridia_Clostridiales_Lachnospira

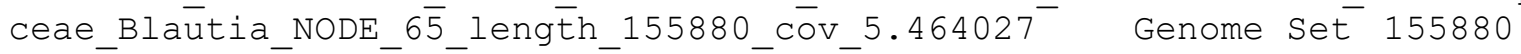

\section{N/A ICE Firmicutes}

UMGS1016_ERR688512_bin.48_Firmicutes_Clostridia_Clostridiales_Lachnospira ceae_Lachnobacteriüm_NODE_192_length_72327_cov_17.659204 Genome Set

$$
72327 \mathrm{~N} / \mathrm{A} \quad \mathrm{ICE} \text { Firmicutes }
$$

NZ_GG730311.1_Clostridium_sp._M62_1_genomic_scaffold_Scfld2_whole_genome shotgun sequenceGenome set $\overline{463016}$ N/A ICE Firmicutes

12718_7_84_Firmicutes_Clostridia_Clostridiales_Ruminococcaceae_NA_.12718_ $784 . \overline{1}-$ Genome Set 559371 - N/A ICE Firmicutes 13414_6_68_Firmicutes_Clostridia_Clostridiales_Lachnospiraceae_NA_.13414_ 6_68.1. $0^{-}$- Genome Set $102183-$ N/A ICE Firmicutes

UMMGS1953_SRR5127782_bin.22_Firmicutes_Clostridia_Clostridiales_NA_NA_NODE

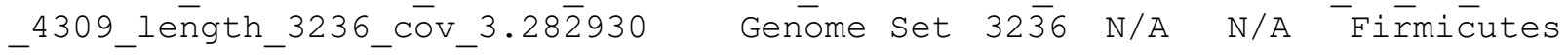


UMGS1654_ERR636353_bin.86_Bacteroidetes_Bacteroidia_Bacteroidales_Bactero

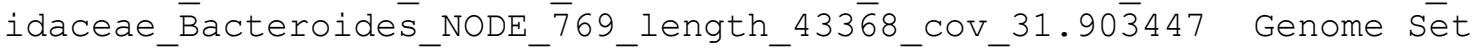
$4 \overline{3} 368 \mathrm{~N} / \mathrm{A} \quad \mathrm{IC} \overline{\mathrm{E}}$ Bācteroidetes

NZ KE340299.1 Propionibacterium sp. HGH0353 genomic scaffold acHMYsupercont1.1_whole_genome_shotgun_sequence Genome Set $273 \overline{5} 781$ N/A ICE Actinobacteria

12718_7_47_Firmicutes_Clostridia_Clostridiales_NA_NA_.12718_7_47.5 Genome set $3548 \overline{3} 3 \quad$ N/A ${ }^{-}$ICE Firmicütes

NZ_JH414706.1_Subdoligranulum_sp._4_3_54A2FAA_genomic_scaffold_supercont1 .9 _whole_genome_shotgun_sequence Genome Set $1729404^{-}$N/A ICE Firmicutes

14207_7_50_Firmicutes_Clostridia_Clostridiales_NA_NA_.14207_7_50.4 Genome set $2289 \overline{4} 1 \quad$ N/A ICE Firmicutes

NC_011593.1_Bifidobacterium_longum_subsp._infantis_ATCC_15697_complete_g enome Genomé Set 2832748 rep_cluster 475 ICE Actinobacteria

21673_4_3_Firmicutes_Clostridia_Clostridiales_Lachnospiraceae_NA_.21673_4 3.12 Genome Set $48307 \mathrm{~N} / \mathrm{A}$ ICE Firmicutes

13470_2_91_Firmicutes_Clostridia_Clostridiales_Lachnospiraceae_NA_.13470_ 2_91.1. ${ }^{-}$- Genome Set $58221 \mathrm{~N} / \mathrm{A}^{-}$ICE Firmicutes

UMMGS38_ERR209883 bin.22_Firmicutes_Clostridia_Clostridiales_Ruminococcace

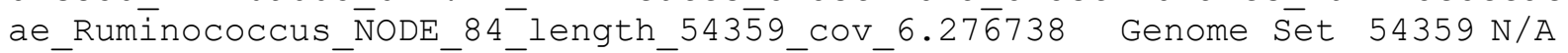
ICE Firmicutes

UMGS1187_ERR011350_bin.8_Firmicutes_Clostridia_Clostridiales_NA_NA_NODE_1 50_length_29933_cov_6.83426 Genome Set 29933 N/A ICE Firmicutes

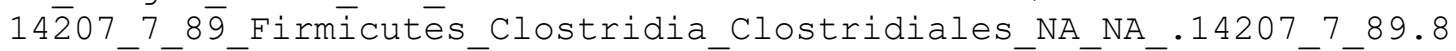
Genome set $1052 \overline{5} 4 \quad$ N/A - ICE Firmicutes

UMGS21_ERR525840_bin.18_Firmicutes_Clostridia_Clostridiales_NA_NA_NODE_11 length_246105_cov_28.59995 Genome set $24610 \overline{5}$ N/A ICE E Firmicutes 20298_3_65_Firmicutes_Clostridia_Clostridiales_Lachnospiraceae_NA_.20298_ 3_65. $\overline{1}-$ Genome Set $307032-$ N/A ICE Firmicutes

NZ_GL520132.1_Erysipelotrichaceae_bacterium_3_1_53_genomic_scaffold_super coñtl.1_wholēegenome_shotgun_sequence Genome $\overline{\text { Set }} 524289^{-}$N/A ${ }^{-}$ICE Tenericutes

13470_2_93_Firmicutes_Clostridia_Clostridiales_Lachnospiraceae_NA_.13470_ 2_93.12- Genome Set 108184 N/A ICE Firmicutes

NZ_KE993553.1_Clostridium_sp._ATCC_29733_genomic_scaffold_Scaffold18_who le_genome_shotgun_sequence Genome set 176422 - N/A ICE Firmicutes UMGS1533_ERR1190600_bin.25_Firmicutes_Clostridia_Clostridiales_Lachnospir

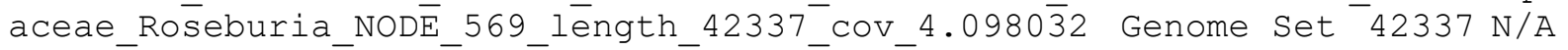
ICE Firmicutes

UMGS1205_ERR209515_bin.8_Bacteroidetes_Bacteroidia_Bacteroidales_Bacteroi

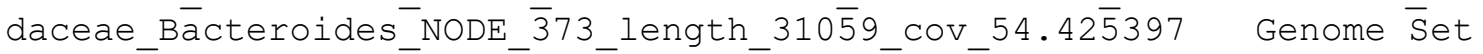
$\overline{3} 1059 \mathrm{~N} / \mathrm{A} \quad \mathrm{IC} E \quad \bar{B}$ actèroidetes

20298_2_28_Firmicutes_Clostridia_Clostridiales_NA_NA_.20298_2_28.4 Genome set $3643 \overline{2} 7 \quad$ N/A

NZ_KQ033914.1_Parabacteroides_goldsteinii_DSM_19448_=WAL_12034_genomiC_S caffold_aczIT-supercont1.3_whole_genome_shotḡun_sequēence Genome Set $3 \overline{8} 9600 \quad \mathrm{~N} / \mathrm{A} \quad \mathrm{ICE}$ - Bacteroidetes

NZ_HG005167.1_Nesterenkonia_massiliensis_strain_NP1_whole_genome_shotgun sēquence Genome set $3486 \overline{6} 4 \quad$ N/A ICE Actinobactería 13414_6_47_Firmicutes_Clostridia_Clostridiales_Lachnospiraceae_Lachnobact

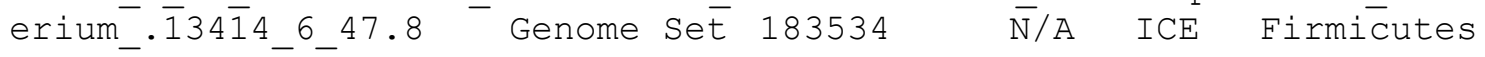


NZ_HE978578.1_Bacteroidales_bacterium_ph8_genomic_scaffold_scaffold00002 whole_genomé_shotgun_sequēnce Genome Set $104 \overline{8151}$ N/A $\quad$ ICE

Bäcteroidetes

UMGS1019 SRR1757434 bin.5 Firmicutes Negativicutes Selenomonadales Veillo

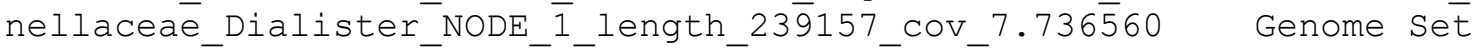
$239 \overline{157}$
$\mathrm{N} / \overline{\mathrm{A}} \quad \mathrm{I} \overline{\mathrm{C}} \mathrm{-}$ Firmicutes

UMGS1617_ERR525879_bin.28_Firmicutes_Clostridia_Clostridiales_Lachnospira ceae_NA_NODE_1801_Iength_177587_cov_7.758841 Genome Set $1758 \overline{7}$ N/A ICE Fírmicūtes

NZ_ACFY01000158.1_Roseburia_inulinivorans_DSM_16841_R_inulinivorans-

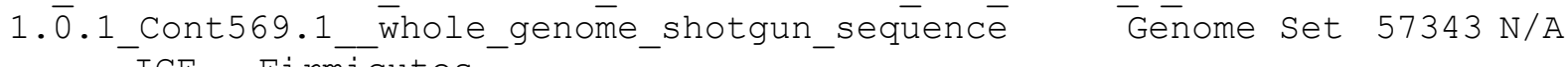
ICE Firmicutes

NZ_AAVM02000003.1_Bacteroides_caccae_ATCC_43185_B_caccae-

MSİQ_Cont1328_whöle_genome_shotgun_sequeñce Gēnome set 500031 N/A ICE Bacteroidetes

NZ_HG726023.1_Bacteroides_neonati_strain_MS4_whole_genome_shotgun_sequen $\mathrm{Ce}^{-}$Genome Set 595790 - N/A ${ }^{-}$ICE Bacteroidetés

13414_6_20_Firmicutes_Clostridia_Clostridiales_Lachnospiraceae_Blautia_.1 $3414 \overline{6} \overline{2} 0 . \overline{1} 1$ Genome Set $120 \overline{295}$ N/A ICE Firmicutes

$1341 \overline{4} \overline{6}$ 32_Firmicutes_Clostridia_Clostridiales_Lachnospiraceae_Blautia_.1 $3414 \overline{6} \overline{3} 2 . \overline{4}$ Genome set $380409-$ N/A ICE Firmicutes

UMGS34 $\overline{1}$ ERR688549_bin.7_Firmicutes_Clostridia_Clostridiales_Peptostreptoc

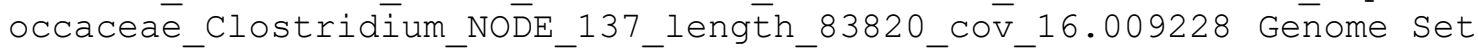
$83 \overline{8} 20 \mathrm{~N} / \mathrm{A} \quad \mathrm{ICE}^{-}$Firmicūtes

14207_7_43_Firmicutes_Clostridia_Clostridiales_Lachnospiraceae_NA_.14207_ $743 . \overline{4}-$ - Genome Set 173976 - N/A ICE Firmicutes

21673_4_53_Firmicutes_Clostridia_Clostridiales_Lachnospiraceae_Blautia_.2 1673 4 $\overline{5} 3 . \overline{1} 1$ Genome Set $133 \overline{4} 73$ N/A ICE Firmicutes

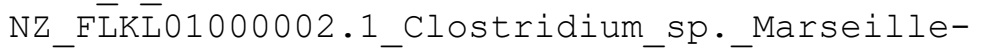

P2538_whole_genome_shotgun_sequence Genome set 4143223 N/A ICE Firmicutes

14207_7_9_Firmicutes_Clostridia_Clostridiales_Lachnospiraceae_Lachnospira $.142 \overline{0} \overline{7}^{-}{ }^{-} 9.13$ Genome set $16 \overline{292} \mathrm{~N} / \mathrm{A}$ ICE Firmicutes

$\bar{N} Z$ AP0 $1 \overline{2} 3 \overline{2} 4.1$ Bifidobacterium_breve_DSM_20213_= JCM_1192_DNA_complete_ge nome Genome Set $2269415 \quad \bar{N} / A \quad \overline{I C E}$ Actinōbācteria

20298_3_36_Firmicutes_Clostridia_Clostridiales_Clostridiales_Family_XI_Pe ptoniphīius_.20298_3_36.1 Genome set 695826 N/A ICE Firmicutes NZ_GG730310.1_Clostríidium_sp._M62_1_genomic_scaffold_Scfld1_whole_genome shotgun_sequenceGenome Set $\overline{648183}$ N/A ICE Firmicutes

UMGS1576_ERR2013621_bin.20_Firmicutes_Clostridia_Clostridiales_Peptostrep

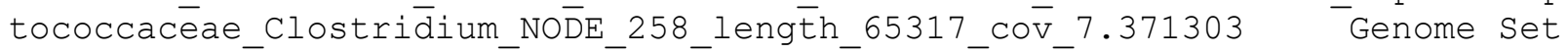
$65317 \overline{\mathrm{N}} / \mathrm{A}$ ICE $\overline{\mathrm{F}}$ irmicutes

8080_1_83_Firmicutes_Clostridia_Clostridiales_Peptostreptococcaceae_Clost

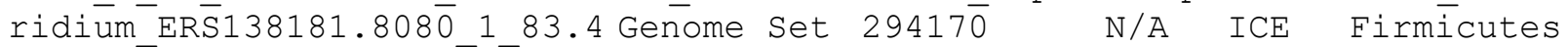
18048 1_70_Firmicutes_Clostridia_Clostridiales_Ruminococcaceae_NA_.18048_ 1_70. $\overline{3}-$ - Genome Set 310645 - N/A ICE Firmicutes 18048_1_70_Firmicutes_Clostridia_Clostridiales_Ruminococcaceae_NA_.18048_ 1_70. ${ }^{-}-$Genome Set 478237 N/A ICE Firmicutes

UM̄GS2027_SRR1761721_bin.40_Bacteroidetes_Bacteroidia_Bacteroidales_Prevot

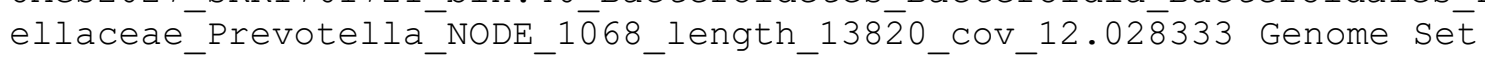

$13 \overline{8} 20 \mathrm{~N} / \mathrm{A}$ ICE Bäcteröidetes

UMGS412_ERR525865_bin.1_Firmicutes_Clostridia_Clostridiales_Lachnospirace

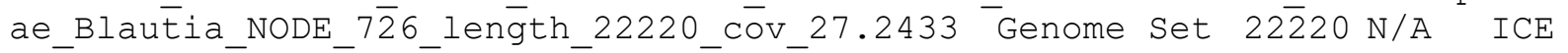
Firmícutes 
UMGS1938_SRR5963258_bin.3_Bacteroidetes_Bacteroidia_Bacteroidales_Porphyr omonadacēae_Dysgonomonas_N̄ODE_98_length_75998_cov_11̄2.586952 Geñome Set $75998 \bar{N} / \mathrm{A} \quad$ ICE Bäteröidētes

UMGS1433 SRR6028647 bin.11 Bacteroidetes Bacteroidia Bacteroidales Prevot

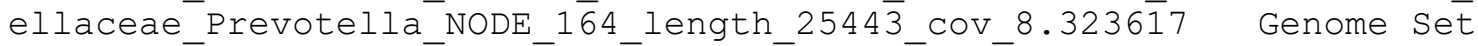

$25 \overline{4} 43 \mathrm{~N} / \mathrm{A} \quad \mathrm{ICE} \quad$ Bäcteroidete $\overline{\mathrm{s}}$

NZ KQ235850.1_[Clostridium] bolteae WAL-

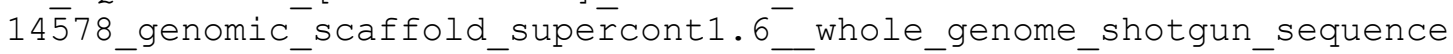

Genome set $33314 \overline{6} \quad$ N/A ICE Firmicutes

UMGS947_SRR1196456_bin.17_Firmicutes_Clostridia_Clostridiales_NA_NA_NODE_

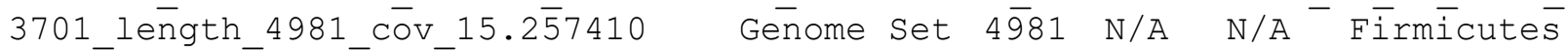

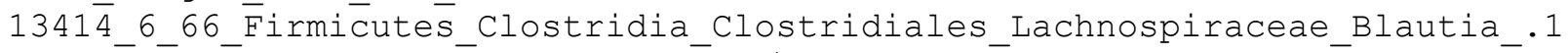
$3414 \overline{6} \overline{6} 6 . \overline{4}$ Genome Set 295338 - N/A ICE Firmicutes

NZ_DS $9 \overline{9} 0131.1$ Bacteroides_plebeius_DSM_17135_Scfld_02_14_genomic_scaffold whole_genoméeshotgun_sequence $\overline{\text { Genome }}$ Set $^{-} 1923 \overline{02}-{ }^{-}$N/A I $\overline{\mathrm{C} E}$

Bacteroidetes

13414_6_32_Firmicutes_Clostridia_Clostridiales_Lachnospiraceae_Blautia_.1 $3414 \overline{6} \overline{3} 2 . \overline{1} 3$ Genome set $800 \overline{1} 8 \mathrm{~N} / \mathrm{A}$ ICE Firmicutes

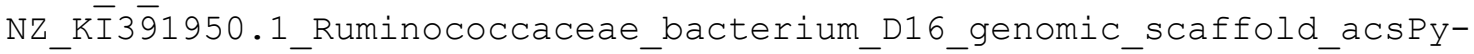
supercont2.4_whole_genome_shōtgun_sequence Genomé Set $141 \overline{1} 41$ N/A ICE Firmicutes

UMGS1210 SRR5106299 bin.4 Bacteroidetes Bacteroidia Bacteroidales Rikenel

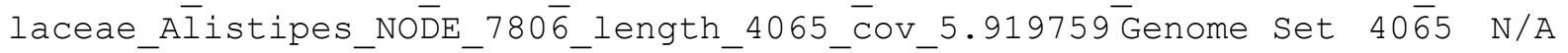
$\overline{\mathrm{N}} / \mathrm{A} \quad$ Bacteroidetes

UMGS793 ERR2013564 bin.23 Firmicutes Clostridia Clostridiales Lachnospira ceae_Psēudobutyrivībrio_NŌDE_59_lengt̄h_102099_cō_6.336708 - Genome Set $102099 \quad \mathrm{~N} / \mathrm{A} \quad \overline{\mathrm{I}} \mathrm{CE}$ Firmicutes

11861_6_61_Bacteroidetes_Bacteroidia_Bacteroidales_Rikenellaceae_Alistipe

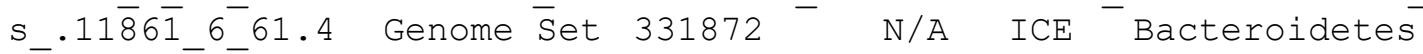

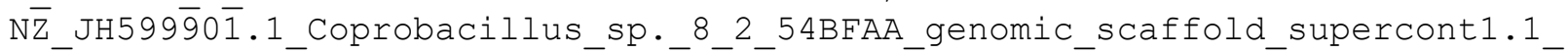
_whole_genome_shotgun_sequence $-{ }^{-}$Genome set $1676 \overline{1} 17$ N/A $\quad$ ICE Tenericutes

NZ_DS990117.1_Bacteroides_plebeius_DSM_17135_Scfld_02_0_genomic_scaffold_ _whole_genome_shotgun_sequence Genome Set $7643^{-}$N/A ${ }^{-}$N/A Bacteroidetes

20427_4_25_Firmicutes_Clostridia_Clostridiales_Lachnospiraceae_Lachnobact erium_. $204 \overline{2} 74425.8$ - Genome Set $134183 \quad \bar{N} / A$ ICE Firmicutes

UMGS $4 \overline{8} 3$ SRR5 $\overline{5} 5 \overline{8} 388$ bin.21_Tenericutes_NA_NA_NA_NA_NODE_14_length_297410_C ov_9.787403 Genome Set $29 \overline{7} 410$ N/A ${ }^{-} \overline{I C E}^{-}$Tenericutes

NZ_ACCL02000022.1_Marvinbryantia_formatexigens_DSM_14469_B_formatexigens-

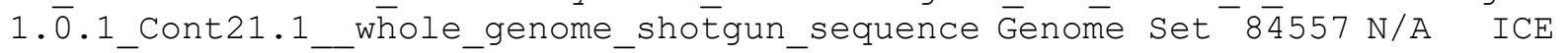
Firmicutes

NZ_KE340303.1_Prevotella_oralis_HGA0225_genomic_scaffold_acAqJ-

supercont1.3_whole_genome_shotgun_sequence Genome Set 291101 N/A ICE Bacteroidetes

NZ_JH379027.1_Hungatella_hathewayi_WAL-

18̄680_supercoñt1.1_wholèe_genome_shotgun_sequence Genome set 3507896

N/A ICE Firmicutes

UMGS1118_SRR341650_bin.4_Bacteroidetes_Bacteroidia_Bacteroidales_Prevotel laceae_Prevotella_NoDE_1310_length_9499__cov_3.62569 Genome Set $9 \overline{499}$ N/A $\overline{\mathrm{N}} / \mathrm{A} \quad$ Bacteroidetes

NZ_FLKM01000009.1_Emergencia_timonensis_strain_SN18_whole_genome_shotgun _séquence Genome set $22889 \overline{3} 3 \quad$ N/A 
NZ_LN913016.1_Anaerococcus_sp._MT16_genome_assembly_Anaerococcus_rubiinfa ntis_scaffold_scaffold00003_whole_genome_shotgun_sequence Genome Set 1764657 rep_cluster 1663 ICE Firmicutes

20287_6_63_Firmicutes_Clostridia_Clostridiales_Lachnospiraceae_NA_.20287_ $663 . \overline{7}-$ Genome Set 166925 N/A ICE Firmicutes

UMGGS1045_SRR3108056_bin.25_Tenericutes_NA_NA_NA_NA_NODE_250_length_68291_

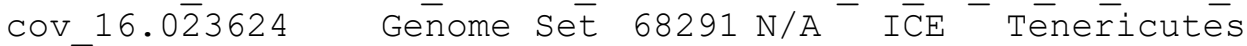

UMGS651_SRR2912799_bin.35_Actinobacteria_Actinobacteria_Coriobacteriales_ Coriobacteriaceae_Collinsēella_NODE_119_lèngth_80866_cov_22.355063

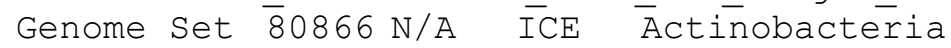

14207_7_54_Firmicutes_Clostridia_Clostridiales_NA_NA_.14207_7_54.12

Genome set $6890 \overline{3}$ N/A ICE Firmicutes

NZ_GL870815.1_Clostridium_sp._D5_genomic_scaffold_supercont1.7_whole_gen omè shotgun séquence Geñome Set $263337^{-}$N/A ${ }^{-}$ICE Firmicutes NZ_THG726024.1_Bacteroides_neonati_strain_MS4_whole_genome_shotgun_sequen $\mathrm{Ce}^{-}$Genome Set 272930 - N/A ICE Bacteroidetés

UMGS566_ERR525865_bin.5_Firmicutes_Clostridia_Clostridiales_Lachnospirace ae_Doreā_NODE_734_length̄22014_cov_58.3496 Genome set 22014 N/A ICE

Firmicutes

20287_6_37_Firmicutes_Clostridia_Clostridiales_NA_NA_.20287_6_37.6

Genome set $1337 \overline{5} 6 \quad$ N/A ICE Firmicütes

UMGS1607_SRR6054490_bin.8_Firmicutes_Clostridia_Clostridiales_Lachnospira ceae_NA_NoDE_496_leñgth_20̄164_cov_6.531006 Genome set $2016 \overline{4}$ N/A ICE Firmicutes

NZ DS981439.1 Bacteroides coprocola DSM 17136 Scfld 0212 genomic scaffol d_whole_genome_shotgun_sēquence Genome set $84241 \overline{\mathrm{N}} / \overline{\mathrm{A}}$ I ICE

Bacteroidetes

UMGS176_SRR2992899_bin.7_Firmicutes_Clostridia_Clostridiales_Ruminococcac

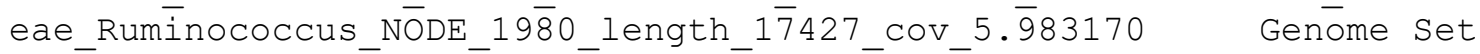
$17427 \mathrm{~N} / \mathrm{A}{ }^{-} \mathrm{N} / \mathrm{A}-$ Firmicutes

11861_6_65_Firmicutes_Clostridia_Clostridiales_Ruminococcaceae_NA_.11861_ $665 . \overline{9}-$ Genome Set 168714 N/A ICE Firmicutes UM̄GS2036_ERR1620302_bin.45_Tenericutes_NA_NA_NA_NA_NODE_3036_length_7999_ cov_2.754406 Genome Set 7999 N/A N/A Ténericutes

UMGS'S20 SRR5057033 bin.5 Firmicutes Clostridia Clostridiales Ruminococcac

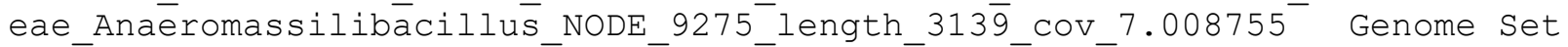
3139 N/A N/A Firmicutes

NZ DS483499.1 Faecalibacterium prausnitzii M21 2 Scfld 0220 genomic scaf fold_whole_genome_shotgun_sequence Geñome sēt $155 \overline{1} 0 \bar{T}^{-}$N/A ICE Firmicutes

20298_3_59_Firmicutes_Clostridia_Clostridiales_NA_NA_.20298_3_59.4 Genome Set 558507 N/A ICE Firmicutes

UMGS1353_ERR589584_bin.12_Firmicutes_Clostridia_Clostridiales_Lachnospira

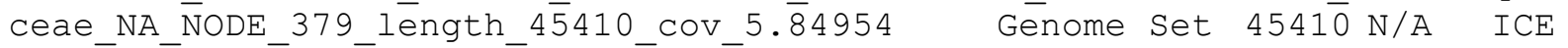
Firmicutes

14207_7_87_Firmicutes_Clostridia_Clostridiales_NA_NA_.14207_7_87.23 Genome set $5160 \overline{6}$ N/A ICE ${ }^{-}$Firmicutes

UMGS963_SRR4305041_bin.1_Bacteroidetes_Bacteroidia Bacteroidales Bacteroi

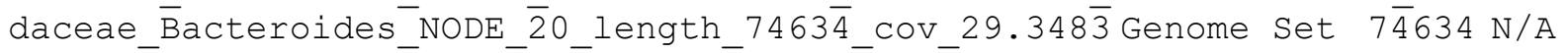
$\bar{I} C E \quad$ Bacteroidetes

UMGS2 ERR1190731_bin.19 Bacteroidetes Bacteroidia Bacteroidales Porphyrom

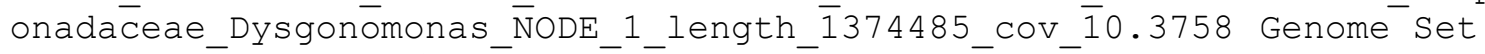
$137 \overline{4} 485 \quad$ N/A ICE Bacteroidetes 
UMGS1355 ERR414360 bin.44 Firmicutes Clostridia Clostridiales Ruminococca

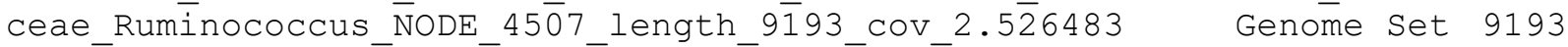
N/A N/A Firmicutes

UMGS1697 SRR5580035 bin.43 Bacteroidetes Bacteroidia Bacteroidales Bacter

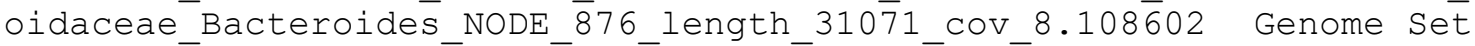

$$
31071 \mathrm{~N} / \mathrm{A} \quad \mathrm{ICE} \text { Bacteroidetes }
$$

UMGS1529_SRR5279269 bin.27 Firmicutes_Clostridia_Clostridiales_Eubacteria

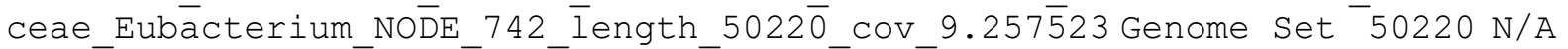
N/A Firmicutes

12718_7_51_Firmicutes_Clostridia_Clostridiales_Lachnospiraceae_Ruminococc us. $1 \overline{2} 7 \overline{1} 8 \overline{7} 51.17 \mathrm{Genome}$ Set $105 \overline{8} 01$ N/A ICE Firmicutes

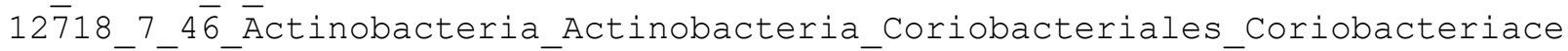

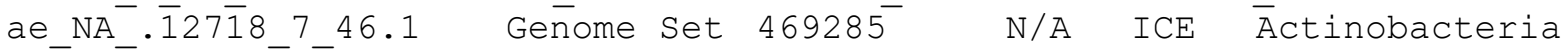
UMGGS828_SRR29992893_bin.21_Firmicutes_Clostridia_Clostridiales_NA_NA_NODE

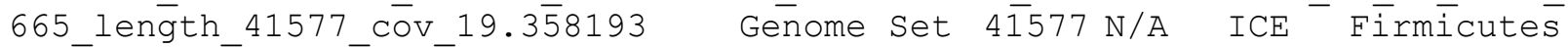
18048_2_85_Firmicutes_Clostridia_Clostridiales_Peptostreptococcaceae_Clos

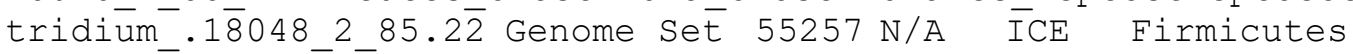

NZ_CCFG01000010.1̄_bacterium_MS4_genome_assembly_Amazonia_massiliensis_co ntíg_contig00010_whole_genōme_shotgun_sequence Genome set 438186
N/A ICE Firmicutes

13470_2_93_Firmicutes_Clostridia_Clostridiales_Lachnospiraceae_NA_.13470_

2 $93 . \overline{2}-$ Genome Set 252339 N/A ICE Firmicutes

13470_2_58_Firmicutes_Clostridia_Clostridiales_NA_NA_.13470_2_58.8

Genome set $1901 \overline{7} 4 \quad$ N/A ${ }^{-}$ICE Firmicutes

UMGS310_SRR4451622_bin.66_Actinobacteria_Actinobacteria_Coriobacteriales_ Coriobacteriaceae_Collinsēlla_NODE_643_lēength_48329_cov_14.516904

Genome set 48329 N/A ICE Áctinobacteria

UMGS1073_ERR1190946_bin.14 Bacteroidetes_Bacteroidia_Bacteroidales_Prevot ellaceae_Paraprevotēlla_NOD̄E_1513_length_33181_cov_8.308036 Genome Set

$33 \overline{1} 81 \mathrm{~N} / \mathrm{A}$ ICE Bacteroidēes

NZ_GL945164.1_Lachnospiraceae_bacterium_1_4_56FAA_genomic_scaffold_superc ont̄1.2_whole_genome_shotgun_sequence Genome Set 505769 N/A ICE Firmicutes

NZ_JH376881.1_Clostridium_clostridioforme_2_1_49FAA_genomic_scaffold_supe rcōnt1.12_whöle_genome_shotgun_sequence $-{ }^{-}$Genome Set $79 \overline{2} 05$ N/A ICE Firmicutes

UMGS1436_ERR1190898_bin.13_Firmicutes_Clostridia_Clostridiales_Lachnospir aceae_Blāutia_NODE_3370_length_7244_cov_17.915844 Genome set 7244 N/A ICE Firmicutes

21673_4_19_Firmicutes_Clostridia_Clostridiales_Lachnospiraceae_Blautia_.2 $1673 \overline{4} \overline{19} . \overline{2} 6$ Genome Set $300 \overline{1}$ N/A ICE Firmicutes $2167 \overline{3} \overline{4}_{4} 46$ Bacteroidetes_Bacteroidia_Bacteroidales_Bacteroidaceae_Bactero

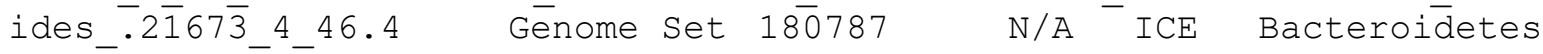
14207_7_59_Firmicutes_Clostridia_Clostridiales_NA_NA_.14207_7_59.12 Genome set $1131 \overline{5} 2$ N/A ICE Firmicütes

14207_7_42_Firmicutes_Clostridia_Clostridiales_Lachnospiraceae_NA_.14207_ $742 . \overline{2} 2^{-}$- Genome Set 80674 N/A ${ }^{-}$ICE Firmicütes NZ̄_FLKM01000008.1_Emergencia_timonensis_strain_SN18_whole_genome_shotgun séquence Genome set $20760 \overline{4} 0$ N/A ICE Firmicutes 14207_7_83_Firmicutes_Clostridia_Clostridiales_Lachnospiraceae_Roseburia_ $.1420 \overline{7} \overline{7}_{-} 8 \overline{3} .41$ Genome set $254 \overline{58}$ N/A ICE Firmicutes 